\title{
I. Fission Probabilities, Fission Barriers, and Shell Effects II. Particle Structure Functions
}

\author{
Kexing Jing \\ Ph.D. Thesis \\ Department of Nuclear Engineering \\ University of California, Berkeley \\ and \\ Nuclear Science Division \\ Ernest Orlando Lawrence Berkeley National Laboratory \\ University of California \\ Berkeley, CA 94720
}

May 1999

This work was supported by the Director, Office of Energy Research, Office of High Energy and Nuclear Physics, of the U.S. Department of Energy under Contract No. DE-AC03-76SF00098. 


\section{DISCLAIMER}

This report was prepared as an account of work sponsored by an agency of the United States Government. Neither the United States Government nor any agency thereof, nor any of their employees, make any warranty, express or implied, or assumes any legal liability or responsibility for the accuracy, completeness, or usefulness of any information, apparatus, product, or process disclosed, or represents that its use would not infringe privately owned rights. Reference herein to any specific commercial product, process, or service by trade name, trademark, manufacturer, or otherwise does not necessarily constitute or imply its endorsement, recommendation, or favoring by the United States Government or any agency thereof. The views and opinions of authors expressed herein do not necessarily state or reflect those of the United States Government or any agency thereof. 


\section{DISCLAIMER}

Portions of this document may be illegible in electronic image products. Images are produced from the best available original document. 


\title{
I. Fission Probabilities, Fission Barriers, and Shell Effects
} II. Particle Structure Functions

\author{
by \\ Kexing Jing \\ B.A. (Sichuan University, Chengdu, China) 1982 \\ M.S. (Chinese Academy of Science, Beijing, China) 1985 \\ A dissertation submitted in partial satisfaction of the \\ requirements for the degree of \\ Doctor of Philosophy \\ in \\ Engineering-Nuclear Engineering \\ in the \\ GRADUATE DIVISION \\ of the \\ UNIVERSITY OF CALIFORNIA, BERKELEY \\ Committee in charge: \\ Professor Stanley G. Prussin, Chair \\ Professor Luciano G. Moretto \\ Professor Jasmina L. Vujic \\ Professor John O. Rasmussen
}

Spring 1999 


\begin{abstract}
I. Fission Probabilities, Fission Barriers, and Shell Effects

II. Particle Structure Functions

by

Kexing Jing

Doctor of Philosophy in Engineering-Nuclear Engineering

University of California, Berkeley

Professor Stanley G. Prussin, Chair
\end{abstract}

In Part I, fission excitation functions of osmium isotopes ${ }^{185,186,187,189}$ Os produced in ${ }^{3} \mathrm{He}+{ }^{182,183,184,186} \mathrm{~W}$ reactions, and of polonium isotopes ${ }^{209,210,211,212} \mathrm{Po}$ produced in ${ }^{3} \mathrm{He} /{ }^{4} \mathrm{He}+{ }^{206,207,208} \mathrm{~Pb}$ reactions, were measured with high precision. These excitation functions have been analyzed in detail based upon the transition state formalism. The fission barriers, and shell effects for the corresponding nuclei are extracted from the detailed analyses.

A novel approach has been developed to determine upper limits of the transient time of the fission process. The upper limits are constrained by the fission probabilities of neighboring isotopes. The upper limits for the transient time set with this new method are $15 \times 10^{-21} \mathrm{sec}$ and $25 \times 10^{-21} \mathrm{sec}$ for Os and Po compound 
nuclei, respectively.

In Part II, we report on a search for evidence of the optical modulations in the energy spectra of alpha particles emitted from hot compound nuclei. The optical modulations are expected to arise from the $\alpha$-particle interaction with the rest of the nucleus as the particle prepares to exit. Some evidence for the modulations has been observed in the alpha spectra measured in the ${ }^{3} \mathrm{He}$-induced reactions, ${ }^{3} \mathrm{He}+$ ${ }^{\text {nat }} \mathrm{Ag}$ in particular. The identification of the modulations involves a technique that substracts the bulk statistical background from the measured alpha spectra, in order for the modulations to become visible in the residuals. Due to insufficient knowledge of the background spectra, however, the presented evidence should only be regarded as preliminary and tentative. 
To YI 


\section{Contents}

List of Figures vi vi

List of Tables $\quad$ ix

I Fission Probabilities, Fission Barriers, and Shell Effects 1

1 Introduction 2

1.1 Transition State Formalism ................... 3

1.2 Extraction of Fission Barriers and Shell Effects from Fission Excitation Functions .......................... 8

1.3 Scaling Laws in Fission Probabilities . . . . . . . . . . . . . . 15

1.4 Transient Time of Fission Process . . . . . . . . . . . . . . 19

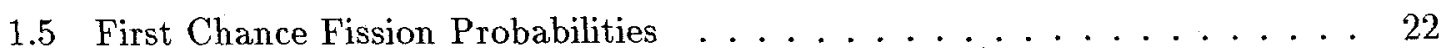

1.6 Goals of the Project . . . . . . . . . . . . . . . 24

2 Measurement of Fission Excitation Functions for Os and Po Isotopes 25

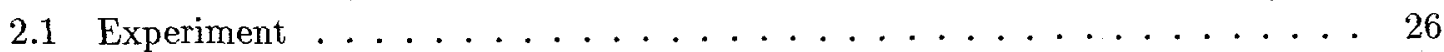

2.2 Fission Cross Sections . . . . . . . . . . . . . 30

3 Fission Barriers and Shell Effects 41

3.1. Formation Cross Sections of the Fissioning Nuclei . . . . . . . . . . . . 42

3.2 Fission Barriers . . . . . . . . . . . . . . . . . . . 48

3.3 Shell Effects . . . . . . . . . . . . . . . . . . 58

3.4 Simultaneous Determination of the Fission Barrier and the Shell Effect . . . 67

3.5 From Fission Cross Sections to Fusion Cross Sections . . . . . . . . . . . 83

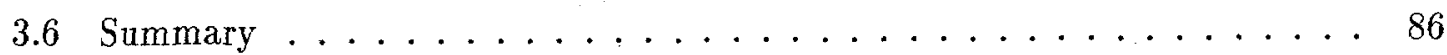

4 Dynamic Fission Time Scale $\quad \mathbf{8 8}$

4.1 Method .............................. 89

4.2 Upper Limits of Fission Transient Times for Os and Po Isotopes $\ldots . . . . \quad 92$

4.3 Simulation of Transient Time Effects on Total Fission Probabilities . . . . . 97

4.4 Resetting the Decay Clock at Each Step in the Decay Chain . . . . . . . . 101 
4.5 First Chance Fission Probabilities . . . . . . . . . . . . . 102

4.6 Summary . . . . . . . . . . . . . . . . 105

$\begin{array}{ll}\text { II Particle Structure Functions } & 106\end{array}$

5 Introduction $\quad 107$

5.1 Particle Structure Functions . . . . . . . . . . . . . . . 108

5.2 Inverse Cross Section . . . . . . . . . . . . . . . . . 112

5.3 Shape Polarization and Evaporation Spectra . . . . . . . . . . . 113

6 Measurement of Particle Spectra 117

7 Tentative Evidence for Particle Structures in Alpha Spectra $\quad 123$

7.1 A Transition State Formalism for Evaporation Spectra . . . . . . . . . . . 124

7.2 Smooth Fit of Alpha Spectra, and Modulation in Residuals . . . . . . . 126

7.2.1 Extracted parameters, and Systematics of Coulomb Barriers for

Alpha Evaporation . . . . . . . . . . . . . . . 133

7.3 Orthogonal Polynomials Representation of Modulations in Alpha Spectra . 141

7.4 An Alternative Approach . . . . . . . . . . . . . . 150

7.5 Conclusion . . . . . . . . . . . . . . . 152

$\begin{array}{ll}\text { Bibliography } & 153\end{array}$

A Generalized Strutinski Smoothing Procedure 159

B Orthogonal Polynomials Analysis and a $\mathrm{C}++$ Code $\quad 163$

B.1 Generation of Orthogonal Polynomials for An Arbitrary Weight Function . 164

B.2 Orthogonal Polynomials Representation of Structural Modulations in

Alpha-Particle Spectra . . . . . . . . . . . . . . 165

B.3 A C++ Code that Implements the Polynomials Analysis Procedure . . . . . 167 


\section{List of Figures}

1.1 Schematic illustration of how the level density is affected by the shell effects of a nucleus . . . . . . . . . . . . . . . . . 10

1.2 Figure illustrating the effect of pairing on the level density . . . . . . . . 12

1.3 The shell effects obtained from the fission excitation functions versus the corresponding values determined from the ground state masses . . . . . . . 14

1.4 The scaling law in fission probabilities ................ 18

1.5 Schematic illustration of the transient time effects on the fission decay width 20

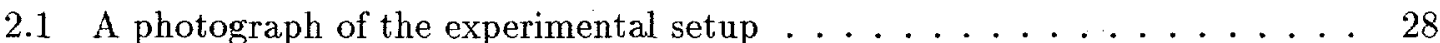

2.2 The fission excitation functions for four Os isotopes produced in the ${ }^{3} \mathrm{He}+$ W reactions . . . . . . . . . . . . . . . . . . . 32

2.3 The fission excitation functions for the Po isotopes produced in the ${ }^{3} \mathrm{He} /{ }^{4} \mathrm{He}$

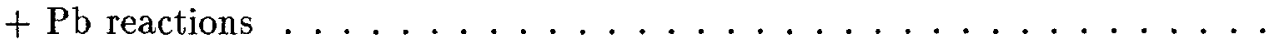

2.4 The ratios of the fission cross sections $\sigma_{f}$ for the ${ }^{3} \mathrm{He} /{ }^{4} \mathrm{He}$-induced fission on ${ }^{\text {nat }} \mathrm{Pb}$ as synthesized from data for individual isotopes and $\sigma_{f}$ measured with a natural lead target . . . . . . . . . . . . . . .

2.5 The ratios of the fission cross sections $\sigma_{f}$ for the ${ }^{3} \mathrm{He}$-induced fission on ${ }^{\text {nat }} \mathrm{W}$ as synthesized from data for individual isotopes and $\sigma_{f}$ measured with a natural tungsten target $\ldots \ldots \ldots \ldots \ldots \ldots$

3.1 Calculated fusion cross sections for the ${ }^{3} \mathrm{He}+{ }^{184} \mathrm{~W}$ reaction . . . . . . . 44

3.2 Calculated fusion cross sections for the ${ }^{3} \mathrm{He}+{ }^{207} \mathrm{~Pb}$ reaction $\ldots \ldots \ldots .45$

3.3 Calculated fusion cross sections for the ${ }^{4} \mathrm{He}+{ }^{207} \mathrm{~Pb}$ reaction $\ldots \ldots \ldots . .46$

3.4 The fits to the fission excitation functions for the Os nuclei produced in the ${ }^{3} \mathrm{He}+\mathrm{W}$ reactions, with $B_{f}^{*}$ and $a_{f} / a_{n}$ as free parameters . . . . . . 52

3.5 The fits to the fission excitation functions for the Po nuclei produced in the ${ }^{3} \mathrm{He}+\mathrm{Pb}$ reactions, with $B_{f}^{*}$ and $a_{f} / a_{n}$ as free parameters $\ldots \ldots \ldots 53$

3.6 The fits to the fission excitation functions for the Po nuclei produced in the ${ }^{4} \mathrm{He}+\mathrm{Pb}$ reactions, with $B_{f}^{*}$ and $a_{f} / a_{n}$ as free parameters $\ldots \ldots \ldots 4$

3.7 The fits to the fission excitation functions for the Os nuclei produced in the ${ }^{3} \mathrm{He}+\mathrm{W}$ reactions, with $\Delta_{\text {shell }}^{n-1}$ and $a_{f} / a_{n}$ as free parameters $\ldots \ldots \ldots$ 
3.8 The fits to the fission excitation functions for the Po nuclei produced in the ${ }^{3} \mathrm{He}+\mathrm{Pb}$ reactions, with $\Delta_{\text {shell }}^{n-1}$ and $a_{f} / a_{n}$ as free parameters . . . . . .

3.9 The fits to the fission excitation functions for the Po nuclei produced in the ${ }^{4} \mathrm{He}+\mathrm{Pb}$ reactions, with $\Delta_{\text {shell }}^{n-1}$ and $a_{f} / a_{n}$ as free parameters $\ldots \ldots 65$

3.10 The fits to the fission excitation functions for the Po nuclei produced in the ${ }^{4} \mathrm{He}+\mathrm{Pb}$ reactions, with $B_{f}^{*}, \Delta_{\text {shell }}^{n-1}$ and $a_{f} / a_{n}$ as free parameters . . . . .

3.11 The fits to the fission excitation functions for the Po nuclei produced in the ${ }^{3} \mathrm{He}+\mathrm{Pb}$ reactions, with $B_{f}^{*}, \Delta_{\text {shell }}^{n-1}$ and $a_{f} / a_{n}$ as free parameters . . . . .

3.12 The fits to the fission excitation functions for the Os nuclei produced in the ${ }^{3} \mathrm{He}+\mathrm{W}$ reactions, with $B_{f}^{*}, \Delta_{\text {shell }}^{n-1}$ and $a_{f} / a_{n}$ as free parameters . . . . .

3.13 The fusion cross sections for the ${ }^{3} \mathrm{He}+\mathrm{Pb}$ reactions obtained by comparing the fission cross sections of the same compound nuclei produced in both the ${ }^{3} \mathrm{He}$-and ${ }^{4} \mathrm{He}$-induced reactions, given the fusion cross sections for the ${ }^{4} \mathrm{He}$ entrance channels as the Bass model prediction . . . . . . . . . . 85

4.1 The simplest assumption for the transient time effects on fission decay rate

4.2 The upper limits of the transient times determined from the fission probabilities of neighboring Os isotopes . . . . . . . . . . . . . . 94

4.3 The upper limits of the transient times determined from the fission probabilities of neighboring Po isotopes . . . . . . . . . . . . . . . 95

4.4 A comparison of the experimental total fission probabilities with the total fission probabilities calculated for a series of assumed transient times . . . 100

5.1 Schematic drawing of the states of a particle in a potential well . . . . . 109

5.2 An optical model calculation with reduced imaginary part of the optical potential . . . . . . . . . . . . . . . . . . . 111

5.3 Normal modes at the saddle point, and the total potential energy and the Coulomb energy as a function of the shape coordinate . . . . . . . . . 114

6.1 The energy spectrum of $\alpha$-particles emitted from the ${ }^{3} \mathrm{He}+{ }^{\text {nat }} \mathrm{Ag}$ reaction at $65 \mathrm{MeV}$ beam energy . . . . . . . . . . . . . . 121

7.1 The fit with Equation 7.3 to the $\alpha$-particle energy spectrum shown in Figure 6.1 , and the percentage difference between the fit and the spectrum ..

7.2 The fits with Equation 7.3 to the energy spectra of $\alpha$-particles emitted from the ${ }^{3} \mathrm{He}+{ }^{\text {nat }} \mathrm{Ag}$ reactions at $55,65,75,85,95,110 \mathrm{MeV}$ beam energies, and the percentage differences between the fits and the spectra . . . . . .

7.3 The fits with Equation 7.3 to the energy spectra of $\alpha$-particles emitted from the ${ }^{3} \mathrm{He}+{ }^{197} \mathrm{Au}$ reactions at $75,85,95,110 \mathrm{MeV}$ beam energies, and the percentage differences between the fits and the spectra . . . . . . .

7.4 The values of the Coulomb barrier $V_{\text {Coul }}^{0}$, the temperature $T$ of the residual nucleus and the amplification parameter $p$, extracted from the fits to the $\alpha$-particle energy spectra for the ${ }^{3} \mathrm{He}+{ }^{\text {nat }} \mathrm{Ag}$ reactions $\ldots \ldots \ldots$ 
7.5 The values of the Coulomb barrier $V_{\text {Coul }}^{0}$, the temperature $T$ of the residual nucleus and the amplification parameter $p$, extracted from the fits to the $\alpha$-particle energy spectra for the ${ }^{3} \mathrm{He}+{ }^{197} \mathrm{Au}$ reactions $\ldots \ldots \ldots \ldots$

7.6 The values of the Coulomb barrier $V_{\text {Coul }}^{0}$, the temperature $T$ of the residual nucleus and the amplification parameter $p$, extracted from the fits to the $\alpha$-particle energy spectra for the ${ }^{3} \mathrm{He}+{ }^{181} \mathrm{Ta}$ reactions . . . . . . . . 137

7.7 The values of the Coulomb barrier $V_{\text {Coul }}^{0}$, the temperature $T$ of the residual nucleus and the amplification parameter $p$, extracted from the fits to the $\alpha$-particle energy spectra for the ${ }^{3} \mathrm{He}+{ }^{\text {nat }} \mathrm{Cu}$ reactions . . . . . . . 138

7.8 The systematics of the Coulomb barriers $V_{\text {Coul }}^{0}$ for $\alpha$-particle evaporations . 139

7.9 The extracted values of the amplification parameter $p$ are plotted versus the atomic number $Z_{\mathrm{CN}}$ of the nucleus from which $\alpha$-particle is emitted . . . 141

7.10 The orthogonal polynomials representation of both the $\alpha$-particle energy spectrum and the residual of the fit for the ${ }^{3} \mathrm{He}+{ }^{\text {nat }} \mathrm{Ag}$ reaction at $65 \mathrm{MeV}$ beam energy . . . . . . . . . . . . . . . . . . . . . . . 144

7.11 The orthogonal polynomials representation of both the $\alpha$-particle energy spectra and the residuals of the fits, for the ${ }^{3} \mathrm{He}+{ }^{\text {nat }} \mathrm{Ag}$ reactions at 55,65 , $75,85,95,110 \mathrm{MeV}$ beam energies . . . . . . . . . . . 146

7.12 The strength $s_{n}$ for a mode in the $\alpha$-particle spectra corresponding to the $n^{\text {th }}$ order polynomial is plotted versus $n$ for the ${ }^{3} \mathrm{He}+{ }^{\text {nat }} \mathrm{Ag}$ reactions . . 147

7.13 The orthogonal polynomials representation of both the $\alpha$-particle energy spectra and the residuals of the fits, for the ${ }^{3} \mathrm{He}+{ }^{197} \mathrm{Au}$ reactions at 75 , $85,95,110 \mathrm{MeV}$ beam energies . . . . . . . . . . . . 148

7.14 The strength $s_{n}$ for a mode in the $\alpha$-particle spectra corresponding to the $n^{\text {th }}$ order polynomial is plotted versus $n$ for the ${ }^{3} \mathrm{He}+{ }^{197} \mathrm{Au}$ reactions . . 149 



\section{List of Tables}

2.1 Thicknesses and isotopic purities of the targets . . . . . . . . . 26

2.2 The measured fission cross sections $\sigma_{f}$ for the Os isotopes produced in the ${ }^{3} \mathrm{He}+\mathrm{W}$ reactions . . . . . . . . . . . . . . . . . . 34

2.3 The measured fission cross sections $\sigma_{f}$ for the Po isotopes produced in the ${ }^{3} \mathrm{He}+\mathrm{Pb}$ reactions . . . . . . . . . . . . . . . . 35

2.4 The measured fission cross sections $\sigma_{f}$ for the Po isotopes produced in the ${ }^{4} \mathrm{He}+\mathrm{Pb}$ reactions . . . . . . . . . . . . . . . 36

3.1 The values of $B_{f}^{*}$ and $a_{f} / a_{n}$ for the Os and Po isotopes extracted from the fits with $\Delta_{\text {shell }}$ fixed with Möller's values . . . . . . . . . . .

3.2 The values of $\Delta_{\text {shell }}^{n-1}$ and $a_{f} / a_{n}$ for the Os and Po isotopes extracted from

the fits with the fission barriers $B_{f}^{*}$ as a fixed parameter . . . . . . . . .
3.3 The values of $B_{f}^{*}, \Delta_{\text {shell }}^{n-1}$ and $a_{f} / a_{n}$, for the Po isotopes produced in the ${ }^{4} \mathrm{He}$ $+\mathrm{Pb}$ reactions, obtained from the fits with all three as free parameters ..

3.4 The values of $B_{f}^{*}, \Delta_{\text {shell }}^{n-1}$ and $a_{f} / a_{n}$, for the Po isotopes produced in the ${ }^{3} \mathrm{He}$ $+\mathrm{Pb}$ reactions, obtained from the fits with all three as free parameters

3.5 The values of $B_{f}^{*}, \Delta_{\text {shell }}^{n-1}$ and $a_{f} / a_{n}$, for the Os isotopes produced in the ${ }^{3} \mathrm{He}$
$+W$ reactions, obtained from the fits with all three as free parameters . . 


\section{Acknowledgements}

I want first to thank Professor Stanley Prussin, for taking me as his student, for his encouragements and his having confidence in me that made my school years a very pleasant experience.

I am greatly indebted to Professor Luciano Moretto, for his guidance. His insights inspired the entire work. Without his insistence, the part II of this thesis would not have come to be.

I owe special thanks to Dr. Gordon Wozniak, for his support and hospitality. I have learned a great deal from him, from his thoroughness, patience, and expertise. I also appreciate the financial support, provided through Moretto/Wozniak group, that facilitated my study in engineering.

Dr. Thorsten Rubehn deserves special mention. He played a major role in the fission experiments. The fission excitation functions reported and analyzed in this thesis are largely his. He also performed the Optical model calculations.

It has been a great pleasure to work with Dr. Larry Phair. His many helps are deeply appreciated.

This work was supported by the Director, Office of the Energy Research, Office of High Energy and Nuclear Physics, of the US Department of Energy under Contract No. DE-AC03-76SF00098. 
Part I

\title{
Fission Probabilities,
}

\author{
Fission Barriers,
}

\author{
and Shell Effects
}




\section{Chapter 1}

\section{Introduction}

A nucleus behaves very much like a liquid drop. A hot nuclear droplet, heated through mechanisms such as compound nucleus formation, will cool itself by either evaporating light particles, or changing its shape and managing to split into two smaller droplets of complementary sizes. The latter process, called nuclear fission, is the main theme of this thesis.

The most probable paths a nucleus may take on the way to fission pass over a saddle point in its potential energy surface. The rate for fission to occur is determined at the saddle point and is controlled by its height. The fission barrier, defined as the saddle point height, thus plays a central role in understanding fission probabilities. The experimental studies of fission probabilities in turn provide us the means to determine the fission barrier.

For a relatively heavy nucleus (lead or heavier) of low-to-moderate excitation energy, fission occurs nearly symmetrically and the two fission fragments are of more 
or less equal size, making fission an apparently distinct process from light particle emission. The fission process, in this case, can be characterized approximately by a single fission barrier.

In general, however, a nucleus can split into a pair of complementary fragments of any mass asymmetry, ranging from very symmetric, such as in fission in its conventional sense, to very asymmetric such as in particle emission. The decay of hot nuclei in the mass region $A \sim 100$ that gives rise to intermediate mass fragments (or complex fragments) exemplifies this general picture. Each mass or charge emission can be associated with a conditional saddle (or barrier) with the constraint of a fixed mass asymmetry. The locus of all such conditional saddles defines the ridge line in the potential energy surface of a nucleus [More 75]. This ridge line controls the emission of complex fragments, and can be measured with techniques similar to those used to determine fission barriers.

\subsection{Transition State Formalism}

The fission rate is often calculated with transition state theory, which was first introduced into chemistry to estimate chemical reaction rates [Wign 38], and was subsequently applied by Bohr and Wheeler to calculate fission probabilities [Bohr 39]. In this approach, the reaction rate is equated to the total flux of phase space trajectories across a dividing surface (a hyperplane) normal to the reaction coordinate. This dividing surface, or the transition state, separates reactants from products, and 
is conventionally located at a saddle point between the reactant and the product region in the potential energy surface of the reacting system. The transition state theory assumes that any phase space trajectory crossing the dividing surface will not find its way back and recross it. The transition state rate is exact only if no trajectory of a given energy crosses the dividing surface more than once. Therefore the transition state location should be so chosen as to minimize the number of phase space trajectories doubling back across the dividing surface.

In the case of fission, the reaction (fission) coordinate can be considered as the deformation coordinate at the saddle point. The fission process can be envisioned as the evolution of the shape of a nucleus from its ground state shape to the saddle point shape, and finally to the scission point where the two halves of the nucleus dissociate into two fission fragments. At the saddle point, the number of all configurations the system can possibly reach becomes minimum, due to the minimization of internal (thermal) energy available to the system. In other words, the saddle point in the potential energy surface corresponds to a bottleneck in phase space.

To determine the fission probability, we consider an ensemble of nuclei, all having excitation energies between $E$ and $E+d E$. The number of nuclei will be chosen to be exactly equal to the number $\rho(E) d E$ of levels in this energy interval, so that there is one nucleus in each state. Let $\lambda_{f}\left(=\Gamma_{f} / \hbar\right)$ be the fission probability per unit time. Then the number of nuclei which fission per unit time will be $\rho(E) d E \cdot \lambda_{f}=$ $\rho(E) d E \cdot\left(\Gamma_{f} / \hbar\right)$. This number will be equal to the number of nuclei in the transition state which pass outward over the fission barrier $B_{f}$ per unit time. In a unit distance 
in the direction of fission there will be $(d p / h) \rho_{s}\left(E-B_{f}-\varepsilon\right) d E$ quantum states for which the momentum $p$ and the kinetic energy $\varepsilon$ associated with fission have values in the intervals $d p$ and $d \varepsilon=v d p$, respectively. Here $d p / h$ is the number of states corresponding to the phase space volume in a unit distance in the fission degree of freedom. $\rho_{s}$ is the density of those levels of the compound nucleus in the transition state (the saddle) which arise from excitation of all degrees of freedom other than the fission itself. At the initial time we have one nucleus in each of the quantum states, and consequently the number of fissions per unit time will be [Bohr 39]

$\rho(E) d E \cdot \frac{\Gamma_{f}}{\hbar}=d E \int \frac{v d p}{h} \rho_{s}\left(E-B_{f}-\varepsilon\right)=d E \int \rho_{s}\left(E-B_{f}-\varepsilon\right) d \varepsilon \cdot \frac{1}{h}=d E N_{s} / h$

where $N_{s}=\int \rho_{s}\left(E-B_{f}-\varepsilon\right) d \varepsilon$ is the total number of levels in the transition state available with given excitation. Then we have

$$
\Gamma_{f}=\frac{N_{s}}{2 \pi \rho(E)}=\frac{1}{2 \pi \rho(E)} \int \rho_{s}\left(E-B_{f}-\varepsilon\right) d \varepsilon
$$

Expanding the logarithm of the level density $\rho_{s}$ around $\left(E-B_{f}\right): \rho_{s}\left(E-B_{f}-\varepsilon\right)$ $\approx \rho_{s}\left(E-B_{f}\right) \exp \left(-\partial\left[\ln \rho_{s}(x)\right] /\left.\partial x\right|_{E-B_{f}} \cdot \varepsilon\right)$, and integrating over $\varepsilon$, one obtains the approximate expression for the fission decay width

$$
\Gamma_{f} \approx \frac{T_{s}}{2 \pi} \frac{\rho_{s}\left(E-B_{f}\right)}{\rho(E)}
$$

where $T_{s}$ is the energy dependent nuclear temperature at the saddle point and $1 / T_{s}=$ $\partial\left[\ln \rho_{s}(x)\right] /\left.\partial x\right|_{E-B_{f}}$

For the one dimensional case, in which the only degree of freedom treated 
explicitly is the reaction coordinate, the decay width takes the form [More 74]

$$
\Gamma_{f} \approx \hbar \omega \frac{\rho_{s}\left(E-B_{f}\right)}{\rho_{s}(E)} \approx \hbar \omega e^{-B_{f} / T}
$$

where $T$ is temperature of the compound nucleus. The quantity $\hbar \omega$ is the oscillator phonon associated with the ground state minimum. In this simplest formulation, one can read the reaction rate in terms of its two factors: the frequency $\omega$ which gives the free rate of assault on the barrier and the Boltzmann factor which gives the probability per assault of making it over the barrier.

This simple and elegant formalism has been successful in many subfields of physics and chemistry, and has become the standard approach to interpret the excitation functions of many physical and chemical processes which require overcoming a potential barrier.

Recent literature, however, provides extensive claims for the failure of transition state rates to account for the amounts of pre-scission neutrons, charged particles, or $\gamma$-rays measured in relatively heavy fissioning systems. These claims prompted our attempts to justify the validity of the transition-state method, and/or to identify regimes in which deviations might be expected. Our attempt in this regard lead to the discovery of a universal scaling law in fission probabilities.

The width for neutron emission which competes very effectively with fission and often dominates the decay process, can also be derived in a very simple form. The Weisskopf formalism [Weis 37] given in the following was derived by taking the transition state to be a spherical shell of unit thickness just outside the nuclear 
surface $4 \pi R^{2}$. The number of quantum states which lie in the transition region and for which the neutron momentum lies in the range $p$ to $p+d p$ and in the solid angle $d \Omega$ will be

$$
\left(4 \pi R^{2} \cdot p^{2} d p d \Omega / h^{3}\right) \cdot g^{\prime} \cdot \rho\left(E-B_{n}-\varepsilon\right) d E
$$

where $B_{n}$ is the neutron separation energy and $\rho\left(E-B_{n}-\varepsilon\right)$ is the level density of the residual nucleus after emission of a neutron with kinetic energy $\varepsilon \cdot g^{\prime}$ is the spin factor $\left(g^{\prime}=2\right)$. We multiply this by the normal velocity $v \cos \theta=(d \varepsilon / d p) \cos \theta$ and integrate over $\Omega$, obtaining the phase space flux passing through the transition state

$$
d E\left(4 \pi R^{2} \cdot 2 \pi m / h^{3}\right) \cdot g^{\prime} \cdot \int \rho\left(E-B_{n}-\varepsilon\right) \varepsilon d \varepsilon
$$

where $m$ denotes the neutron mass. This is the number of neutron emissions occurring per unit time that is to be indentified with $\rho(E) d E \cdot \lambda_{n}=\rho(E) d E \cdot\left(\Gamma_{n} / \hbar\right)$. Therefore we have for the probability of neutron emission, expressed in energy units,

$$
\Gamma_{n}=\frac{2 m R^{2} g^{\prime}}{\hbar^{2}} \frac{1}{2 \pi \rho(E)} \int \varepsilon \rho\left(E-B_{n}-\varepsilon\right) d \varepsilon
$$

Expanding the logarithm of the level density $\rho$ around $\left(E-B_{n}\right): \rho\left(E-B_{n}-\varepsilon\right)$ $\approx \rho\left(E-B_{n}\right) \exp \left(-\partial[\ln \rho(x)] /\left.\partial x\right|_{E-B_{n}} \cdot \varepsilon\right)$ and integrating over $\varepsilon$, one obtains the approximate expression for the neutron decay width

$$
\Gamma_{n} \approx K T_{n}^{2} \frac{\rho\left(E-B_{n}\right)}{2 \pi \rho(E)}
$$

where $T_{n}$ is the temperature of the residual nucleus after neutron emission defined as $1 / T_{n}=\partial[\ln \rho(x)] /\left.\partial x\right|_{E-B_{n}}$, and $K=2 m R^{2} g^{\prime} / \hbar^{2}$. 
More sophisticated formalisms such as the Hauser-Feshbach formalism [Haus 52] could also be used to evaluate the neutron width. The Hauser-Feshbach formalism, however, necessarily introduces additional unknowns - the transmission coefficients $T_{\ell}$ which have to be calculated with specific models. The Weisskopf formalism, on the other hand, emphasizes phase space, and is therefore less model specific. In both the Weisskopf and the Hauser-Feshbach formalisms, the common (dominant) factor is the same, i.e., the level density of the residual nucleus after a neutron emission. For simplicity and transparency we choose to use the Weisskopf formalism in our analysis of the fission excitation functions.

\subsection{Extraction of Fission Barriers and Shell Effects from}

\section{Fission Excitation Functions}

As can be seen in Eq. 1.3, the fission barrier $B_{f}$ controls the fission decay width, especially at low energies. Thus a detailed study of the experimentally measured fission cross sections as a function of excitation energy should allow us to determine the fission barriers.

Taking into account the angular momentum a fissioning nucleus may have, the fission decay width can be rewritten as

$$
\Gamma_{f} \approx \frac{T_{s}}{2 \pi} \frac{\rho_{s}\left(E-B_{f}-E_{r}^{s}\right)}{\rho\left(E-E_{r}^{g s}\right)}
$$

where $E_{r}^{s}$ and $E_{r}^{g s}$ denote the rotational energy of the system at the saddle point and the energy of the rotating ground state relative to the non-rotating macroscopic 
sphere. Accordingly, the width for neutron emission can be rewritten as

$$
\Gamma_{n} \approx K T_{n}^{2} \frac{\rho\left(E-B_{n}-E_{r}^{g s}\right)}{2 \pi \rho\left(E-E_{r}^{g s}\right)}
$$

Using for simplicity the Fermi gas level density

$$
\rho(E) \propto \exp (2 \sqrt{a E})
$$

where $a$ is the level density parameter, $\Gamma_{f}$ and $\Gamma_{n}$ can then be evaluated readily. In particular, by integrating directly Eqs. $1.2 \& 1.7$, one obtains for $\Gamma_{f} / \Gamma_{n}$ :

$$
\frac{\Gamma_{f}}{\Gamma_{n}}=\frac{T_{s}-\frac{1}{2 a_{f}}}{K\left(T_{n}^{2}-\frac{3}{2 a_{n}} T_{n}+\frac{3}{4 a_{n}^{2}}\right)} \frac{\rho_{s}\left(E-B_{f}-E_{r}^{s}\right)}{\rho\left(E-B_{n}-E_{r}^{g s}\right)}
$$

or more explicitly:

$$
\frac{\Gamma_{f}}{\Gamma_{n}}=\frac{T_{s}-\frac{1}{2 a_{f}}}{K\left(T_{n}^{2}-\frac{3}{2 a_{n}} T_{n}+\frac{3}{4 a_{n}^{2}}\right)} \exp \left(2 \sqrt{a_{f}\left(E-B_{f}-E_{r}^{s}\right)}-2 \sqrt{a_{n}\left(E-B_{n}-E_{r}^{g s}\right)}\right),
$$

where $a_{f}$ and $a_{n}$ denote the level density parameter at the saddle and the level density parameter for the residual nucleus after neutron emission, respectively.

For nuclei with strong shell effects (nuclei in the lead region, for example), however, the approximation $\rho\left(E-B_{n}-E_{r}^{g s}\right) \propto \exp \left(2 \sqrt{a_{n}\left(E-B_{n}-E_{r}^{g s}\right)}\right)$ becomes a poor one. The shell effects of a nucleus affect its level density in a rather complicated way at very low energies. At excitation energies of a few $\mathrm{MeV}$ or more above the shell effects $\Delta_{\text {sh }}$, however, the level density assumes its asymptotic form $\rho(E) \propto$ $\exp \left(2 \sqrt{a_{n}\left(E-\Delta_{\text {sh }}\right)}\right)$ [Rose 57 , Huiz 72]. This is shown schematically in Fig. 1.1, 


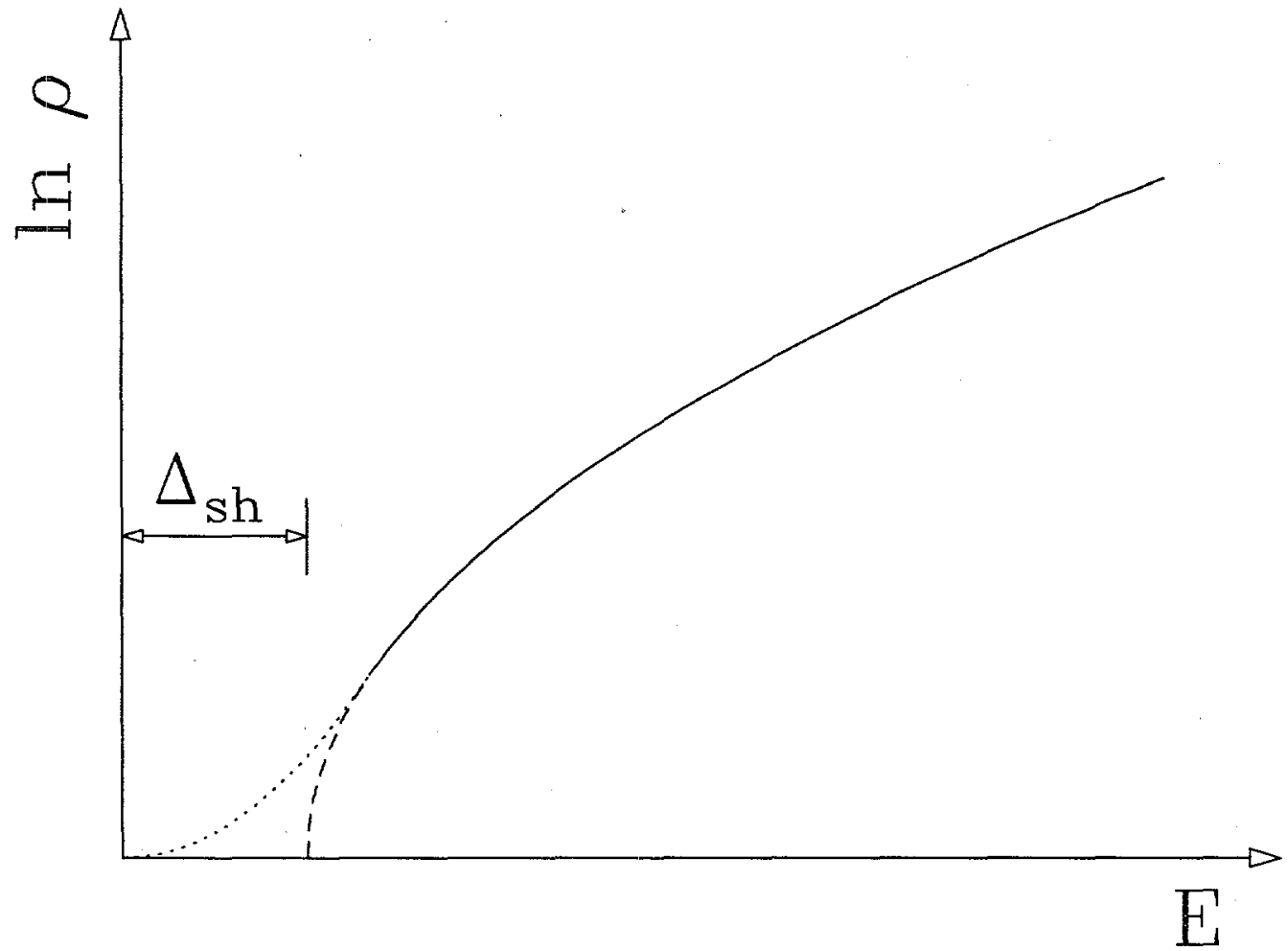

Figure 1.1: The logarithm of the level density $\ln \rho$ is plotted, schematically, versus the internal excitation energy $E$, for a nucleus with shell effects $\Delta_{\mathrm{sh}}$.

where the logarithm of the level density $\ln \rho(E)$ for a nucleus with shell effects $\Delta_{\mathrm{sh}}$ is plotted versus the internal excitation energy $E$. For the daughter nucleus produced by neutron emission from a nucleus in the lead region, when the excitation energy of the daughter nucleus is greater than $15-20 \mathrm{MeV}$, the level density thus takes the asymptotic form:

$$
\rho\left(E-B_{n}-E_{r}^{g s}\right) \propto \exp \left(2 \sqrt{a_{n}\left(E-B_{n}-E_{r}^{g s}-\Delta_{\text {shell }}^{n-1}\right)}\right)
$$

where $\Delta_{\text {shell }}^{n-1}$ is the ground state shell effect of the daughter nucleus after neutron emission. For the level density at the saddle point $\rho_{s}$, the problems should be far less serious. On the one hand, the large deformations at the saddle point imply small 
shell effects. On the other hand, by its nature the saddle locates itself in between maxima and minima in the potential energy surface.

The pairing effects of a nucleus also affect its level density, in a manner similar to that with which the shell effects affect the level density. In Fig. 1.2, the logarithm of the level density is plotted schematically versus the internal excitation energy for a nucleus of pairing condensation energy $\Delta E_{c}$. For an even-even nucleus, $\Delta E_{c}=\frac{1}{2} g \Delta_{0}^{2} ;$ and for an odd $A$ nucleus, $\Delta E_{c}=\frac{1}{2} g \Delta_{0}^{2}-\Delta_{0}$, where $\Delta_{0}$ is the gap parameter and $g$ is the density of doubly-degenerate single particle levels. At excitation energies of a few $\mathrm{MeV}$ or more, the level density also assumes its asymptotic form [Sano 63, Eric 60]:

$$
\begin{aligned}
& \rho(E) \propto \exp \left(2 \sqrt{a\left(E-\frac{1}{2} g \Delta_{0}^{2}\right)}\right), \quad \text { for even-even } \\
& \rho(E) \propto \exp \left(2 \sqrt{a\left(E-\frac{1}{2} g \Delta_{0}^{2}+\Delta_{0}\right)}\right), \quad \text { for odd } A .
\end{aligned}
$$

For the level density at the saddle point $\rho_{s}$, deviations due to pairing may also be expected at very low excitation energies. However, it should be safe to use:

$$
\left.\rho_{s}\left(E-B_{f}-E_{r}^{s}\right) \propto \exp \left(2 \sqrt{a_{f}\left(E-B_{f}^{*}-E_{r}^{s}\right.}\right)\right),
$$

where $B_{f}^{*}=B_{f}+\frac{1}{2} g_{s} \Delta_{s}^{2}$ for even-even nuclei and $B_{f}^{*}=B_{f}+\frac{1}{2} g_{s} \Delta_{s}^{2}-\Delta_{s}$ for odd $A$ nuclei. $\Delta_{s}$ is the saddle gap parameter and $g_{s}$ is the density of doubly-degenerate single particle levels at the saddle. In other words, $B_{f}^{*}$ represents the unpaired saddle energy.

The correction for the pairing effects on the extracted fission barriers is not attempted in this thesis because of its great uncertainties. For a fissioning even-even 


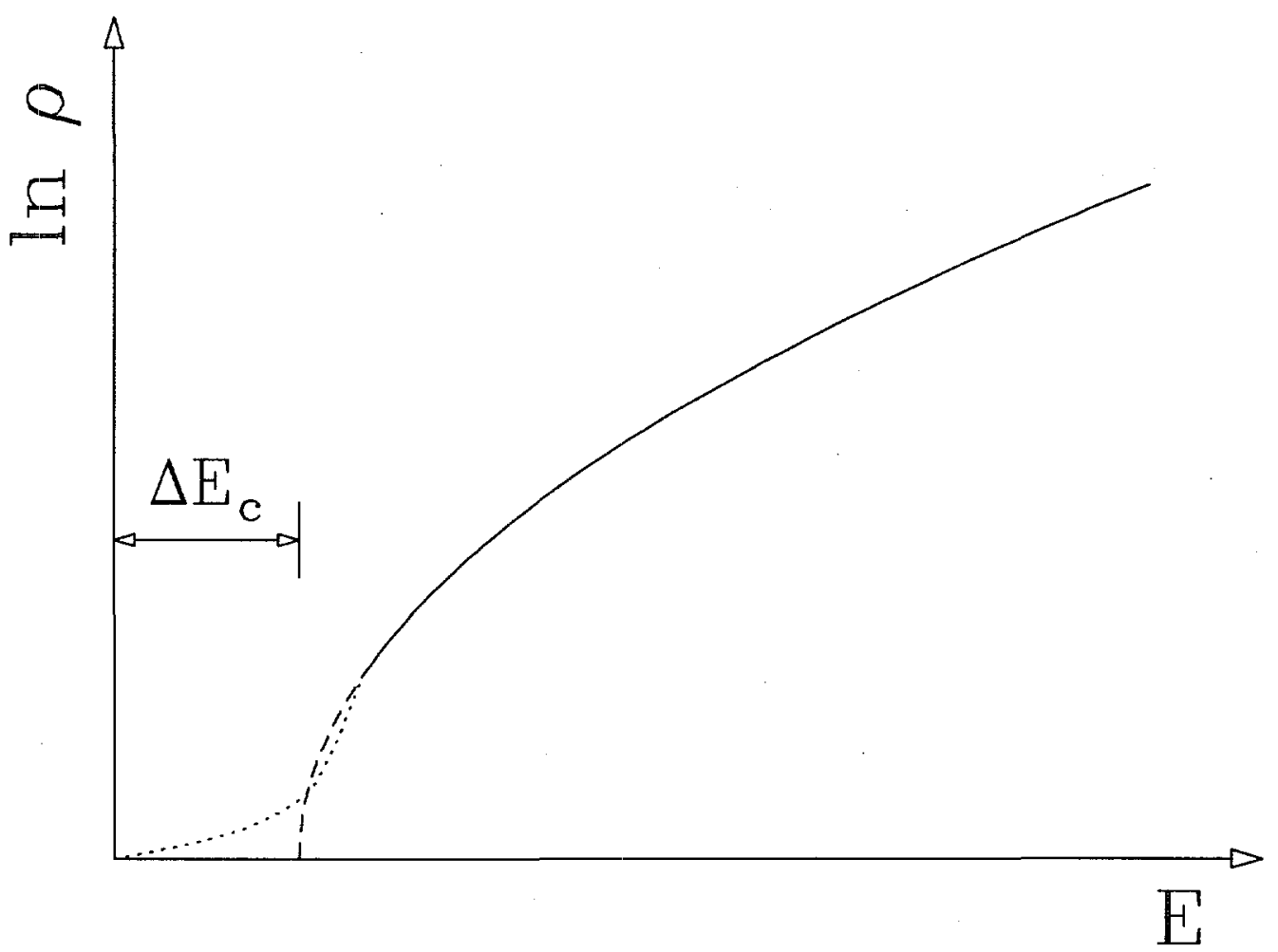

Figure 1.2: The logarithm of the level density $\ln \rho$ is plotted, schematically, versus the internal excitation energy $E$ [Sano 63], for a nucleus with a pairing condensation energy $\Delta E_{\mathrm{c}}$. For an even-even nucleus, $\Delta E_{c}=\frac{1}{2} g \Delta_{0}^{2}$; and for an odd $A$ nucleus, $\Delta E_{c}=\frac{1}{2} g \Delta_{0}^{2}-$ $\Delta_{0}$, where $\Delta_{0}$ is the gap parameter and $g$ the density of doubly degenerate single particle levels.

nucleus, the effect of the pairing on the extracted barriers may be estimated in the following way:

$$
\begin{aligned}
\frac{\Gamma_{f}}{\Gamma_{\text {total }}} \approx \frac{\Gamma_{f}}{\Gamma_{n}} \sim & \frac{\rho\left(E-B_{f}-\frac{1}{2} g_{s} \Delta_{s}^{2}-E_{r}^{s}\right)}{\rho\left(E-B_{n}-\Delta_{\text {shell }}^{n-1}-\frac{1}{2} g_{n} \Delta_{n}^{2}+\Delta_{n}-E_{r}^{g s}\right)} \\
& \approx \frac{\rho\left(E-\left(B_{f}^{*}+\frac{T_{s}}{T_{n}}\left(\frac{1}{2} g_{n} \Delta_{n}^{2}-\Delta_{n}\right)\right)-E_{r}^{s}\right)}{\rho\left(E-B_{n}-\Delta_{\text {shell }}^{n-1}-E_{r}^{g s}\right)}
\end{aligned}
$$

where $\Delta_{n}$ is the gap parameter of the residual nucleus after neutron emission, and $g_{n}$ is the density of doubly-degenerate single particle levels of the residual nucleus. Here all the level densities have been implicitly assumed to take the form of the Fermi gas 
level density. Similarly, for an even $Z$ but odd $A$ fissioning nucleus,

$$
\begin{aligned}
\frac{\Gamma_{f}}{\Gamma_{\text {total }}} \approx \frac{\Gamma_{f}}{\Gamma_{n}} \sim \frac{\rho\left(E-B_{f}-\frac{1}{2} g_{s} \Delta_{s}^{2}+\Delta_{s}-E_{r}^{s}\right)}{\rho\left(E-B_{n}-\Delta_{\text {shell }}^{n-1}-\frac{1}{2} g_{n} \Delta_{n}^{2}-E_{r}^{g s}\right)} \\
\approx \frac{\rho\left(E-\left(B_{f}^{*}+\frac{T_{s}}{T_{n}}\left(\frac{1}{2} g_{n} \Delta_{n}^{2}\right)\right)-E_{r}^{s}\right)}{\rho\left(E-B_{n}-\Delta_{\text {shell }}^{n-1}-E_{r}^{g s}\right)} .
\end{aligned}
$$

In the above equations, the pairing effects at the saddle are incorporated into the effective fission barrier $B_{f}^{*}$. To correct for the pairing effects of the residual nucleus, the extracted barrier $B_{f}^{*}$ should be shifted down by $\sim\left\langle T_{s} / T_{n}\right\rangle \Delta E_{c}$. This average should correspond to the excitation energy where the excitation functions are steepest. Thus it is expected that $\left\langle T_{s} / T_{n}\right\rangle \ll 1$ and the correction should be of the order of $\sim 1 \mathrm{MeV}$ or less.

Let $\sigma_{\ell}$ be the angular momentum distribution of the fusion cross section. Then the fission cross section $\sigma_{f}$ is:

$$
\sigma_{f}=\sum_{0}^{\ell_{\max }} \sigma_{\ell} P_{f}(\ell)
$$

where $P_{f}(\ell)$ is the fission probability of the nucleus of angular momentum $\ell(\hbar)$ and $\ell_{\max }$ is the maximum of angular momenta brought into the compound nucleus by the entrance channel reaction. For first chance fission, $P_{f}(\ell)=\Gamma_{f} / \Gamma_{\text {total }} \approx \Gamma_{f} / \Gamma_{n}$, where $\Gamma_{\text {total }}=\Gamma_{n}+\Gamma_{f}+\cdots$ is the total decay width. Multichance fission can also be conveniently included in the expression for $P_{f}(\ell)$.

Thus, by fitting the experimentally measured fission excitation functions with Eq. 1.20 , using $B_{f}^{*}$ and $\Delta_{\text {shell }}^{n-1}$ as free parameters, one determines the values of 


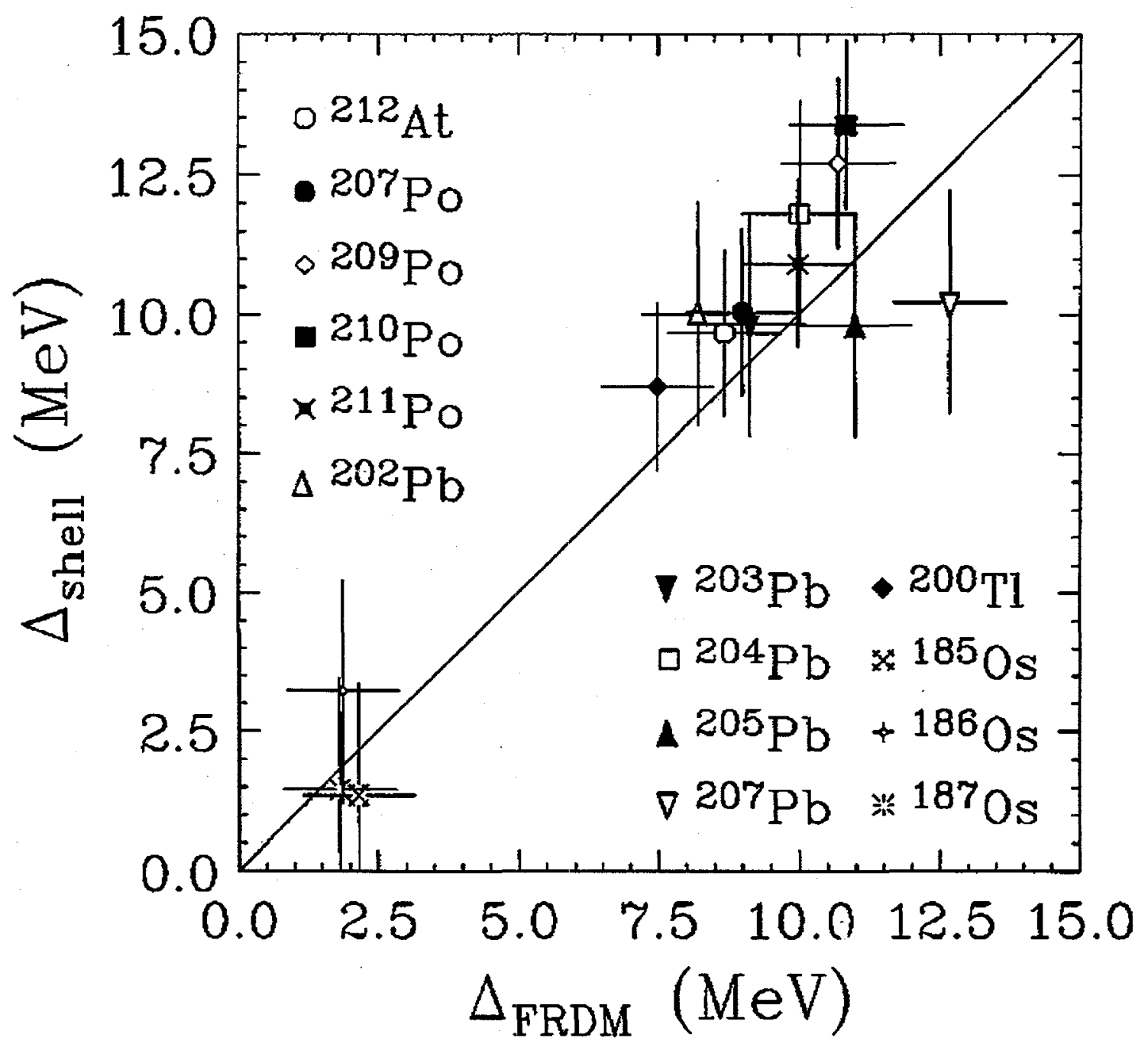

Figure 1.3: Shell effects $\Delta_{\text {shell }}$ for daughter nuclei produced by neutron evaporation, extracted from fits to the fission excitation functions [More 95b], are plotted against the values determined from the ground state masses [Möll 93]. The diagonal line is to guide the eye. 
the effective fission barrier $B_{f}^{*}$ and the shell effect $\Delta_{\text {shell }}^{n-1}$ of the daughter nucleus after neutron emission.

This new way of determining the shell effects, in contrast to the standard procedure [Möll 93, Myer 94, Myer 96], is completely local. It depends only on the properties of the nucleus under consideration. Shown in Fig. 1.3 are the shell effects extracted from fits to the fission excitation functions for fourteen nuclei in the lead region plotted against the corresponding values obtained as the difference of the ground state mass and the corresponding liquid drop value [Möll 93]. The correlation between the shell effects extracted by this method and those obtained from the ground state masses is excellent.

\subsection{Scaling Laws in Fission Probabilities}

Let $\sigma_{0}$ be the compound nucleus formation cross section. The transition state fission cross section can then be expressed as

$$
\sigma_{f}=\sigma_{0} \frac{\Gamma_{f}}{\Gamma_{\text {total }}} \approx \sigma_{0} \frac{1}{\Gamma_{\text {total }}} \frac{T_{s} \rho_{s}\left(E-B_{f}\right)}{2 \pi \rho(E)}
$$

This equation can be rewritten as:

$$
\frac{\sigma_{f}}{\sigma_{0}} \Gamma_{\text {total }} \frac{2 \pi \rho(E)}{T_{s}}=\rho_{s}\left(E-B_{f}\right)
$$

By evaluating the left hand side of this equation, we obtain, on the right hand side, the level density of the system at the saddle point. A new quantity $R_{f}$, called the 
reduced fission probability, is naturally introduced:

$$
R_{f} \equiv \frac{\sigma_{f}}{\sigma_{0}} \Gamma_{\text {total }} \frac{2 \pi \rho(E)}{T_{s}}
$$

Thus the reduced fission probability is equal to the level density at the saddle point,

$$
R_{f}=\rho_{s}\left(E-B_{f}\right)
$$

In general, the compound nucleus formed in a fusion reaction has a distribution of angular momenta. The compound nucleus formation cross section is the sum of all partial fusion cross section $\sigma_{\ell}$ with angular momentum $\ell$,

$$
\sigma_{0}=\sum_{0}^{\ell_{\max }} \sigma_{\ell}
$$

In this general case, if the angular momenta of the fissioning nuclei are not too large, an equation similar to Eq. 1.22 may be written for the compound nucleus formed in a fusion reaction, to a first order approximation as

$$
\frac{\sigma_{f}}{\sigma_{0}} \frac{\left\langle\Gamma_{\text {total }} 2 \pi \rho\left(E-E_{r}^{g s}\right)\right\rangle}{\left\langle T_{s}\right\rangle}=\left\langle R_{f}\right\rangle=\rho_{s}\left(E-B_{f}-\left\langle E_{r}^{s}\right\rangle\right),
$$

where $\left\langle E_{r}^{s}\right\rangle$ and $\left\langle E_{r}^{g s}\right\rangle$ are the average rotational energy of the fissioning nucleus at the saddle point and the average energy of the rotating ground state, respectively. Assuming a sharp cut-off at $\ell_{\max }$ for the angular-momentum distribution, $\left\langle E_{r}^{s}\right\rangle$ and $\left\langle E_{r}^{g s}\right\rangle$ can be evaluated as the rotational energy at the saddle and the energy of the rotating ground state corresponding to $\left\langle\ell^{2}\right\rangle=\ell_{\max }^{2} / 2$, respectively.

Using the Fermi gas level density, we obtain:

$$
\ln \left\langle R_{f}\right\rangle=2 \sqrt{a_{f}\left(E-B_{f}^{*}-\left\langle E_{r}^{s}\right\rangle\right)} .
$$


Thus, plotting the logarithm of the reduced fission probability, $\ln \left\langle R_{f}\right\rangle$, versus the square root of the internal excitation energy $\sqrt{E-B_{f}^{*}-\left\langle E_{r}^{s}\right\rangle}$, we should obtain a straight line.

The slope of the straight line given by Eq. 1.27 depends on the mass of the fissioning nucleus via the level density parameters $a_{n}$ and $a_{f}$. Taking out the mass dependence of the level density parameters, one obtains:

$$
\frac{\ln \left\langle R_{f}\right\rangle}{2 \sqrt{a_{n}}}=\sqrt{\frac{a_{f}}{a_{n}}\left(E-B_{f}^{*}-\left\langle E_{r}^{s}\right\rangle\right)} .
$$

This equation should permit us to scale all the fission excitation functions onto a single universal straight line.

In Fig. 1.4, the left-hand side of the equation 1.28 is plotted versus the square root of the internal excitation energy $\sqrt{E-B_{f}^{*}-\left\langle E_{r}^{s}\right\rangle}$, for fourteen different compound nuclei in the lead region. All of these excitation functions reduce nicely to a single straight line. This scaling extends well over seven orders of magnitude in fission probabilities. The straight line, which is a linear fit to all but the lowest data points, passes through zero very closely, and its slope is nearly $45^{\circ}$ indicating that the ratio $a_{f} / a_{n}$ is very close to unity. The universality of the scaling and the lack of deviation from a straight line over the entire energy range, except for the very lowest energies, indicates that the transition state formalism holds extremely well.

The linear scaling laws are also observed by the mass-asymmetric fission probabilities of nuclei in the intermediate mass region, where over 90 different excitation functions for complex fragments arising from mass-asymmetric fission of the 


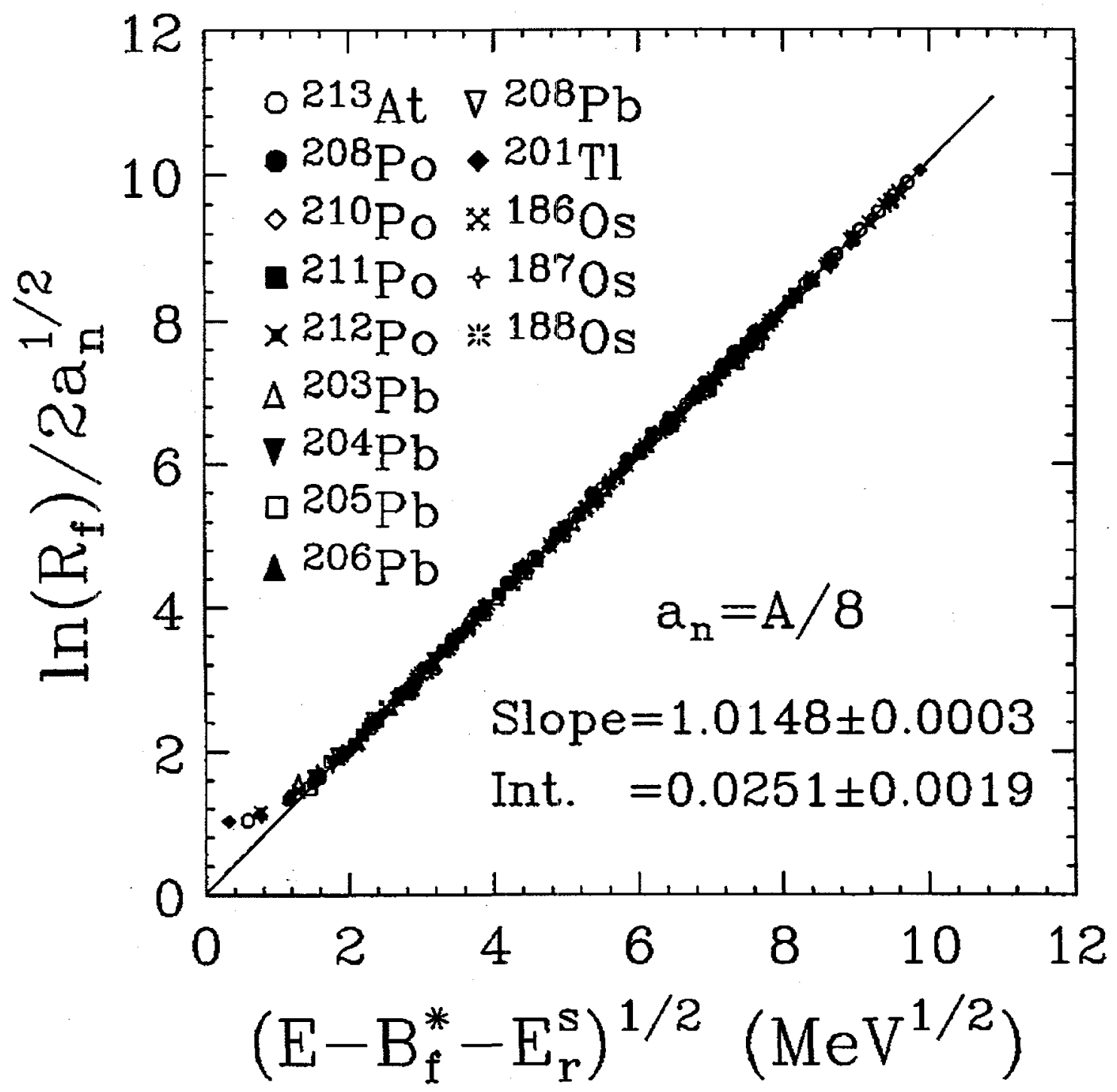

Figure 1.4: The quantity $\ln R_{f}$ divided by $2 \sqrt{a_{n}}$ versus the square root of the intrinsic excitation energy over the saddle point for fission of the compound nuclei: ${ }^{186,187,188} \mathrm{Os}$, ${ }^{201} \mathrm{Tl},{ }^{203,204,205,206,208} \mathrm{~Pb},{ }^{208,210,211,212} \mathrm{Po}$, and ${ }^{213} \mathrm{At}$ [More 95b]. The straight line is a linear fit to all but lowest two or three data points. 
compound nuclei ${ }^{75} \mathrm{Br},{ }^{90,94,98} \mathrm{Mo}$, and ${ }^{110-112}$ In were shown to be scalable onto a single universal straight line according to transition state predictions. More details on this work are given in the papers [Jing 99, More 95a].

\subsection{Transient Time of Fission Process}

The evolution of a fissioning system, starting from an assumed spherical shape towards the fission saddle, and eventually to the scission point, has been studied extensively [Hils 92, Paul 94, Frob 97]. If the transient time that a nucleus takes to evolve from a ground state shape to the saddle point is longer than the characteristic time for compound nucleus $(\mathrm{CN})$ decay $\left(\tau_{\mathrm{CN}}=\hbar / \Gamma_{\mathrm{CN}} \approx \hbar /\left(\Gamma_{f}+\Gamma_{n}\right)\right)$, then fission probabilities are expected to be suppressed, and additional particles can be emitted, as compared to those predicted by transition state theory. This is shown schematically in Fig. 1.5, where the fission width is plotted versus time [Gran 83]. If a transient time $\tau_{D}$ is short as compared to the compound nucleus decay time $\tau_{\mathrm{CN}}$, the stationary Kramers current [Kram 40] (i.e., the transition state fission rate) is then expected. If a transient time is long as compared to $\tau_{\mathrm{CN}}$, then the fission width is necessarily surpressed.

This transient time effect has been advocated as an explanation for the large number of prescission neutrons [Gavr 81, Holu 83, Hind 92, Fior 93], charged particles [Scha 84, Peas 88, Siwe 87], and electric dipole $\gamma$-rays [Paul 94, Thoe 87] observed in relatively heavy fissioning systems. Transient times as long as $\sim 10^{-19}$ sec have been 


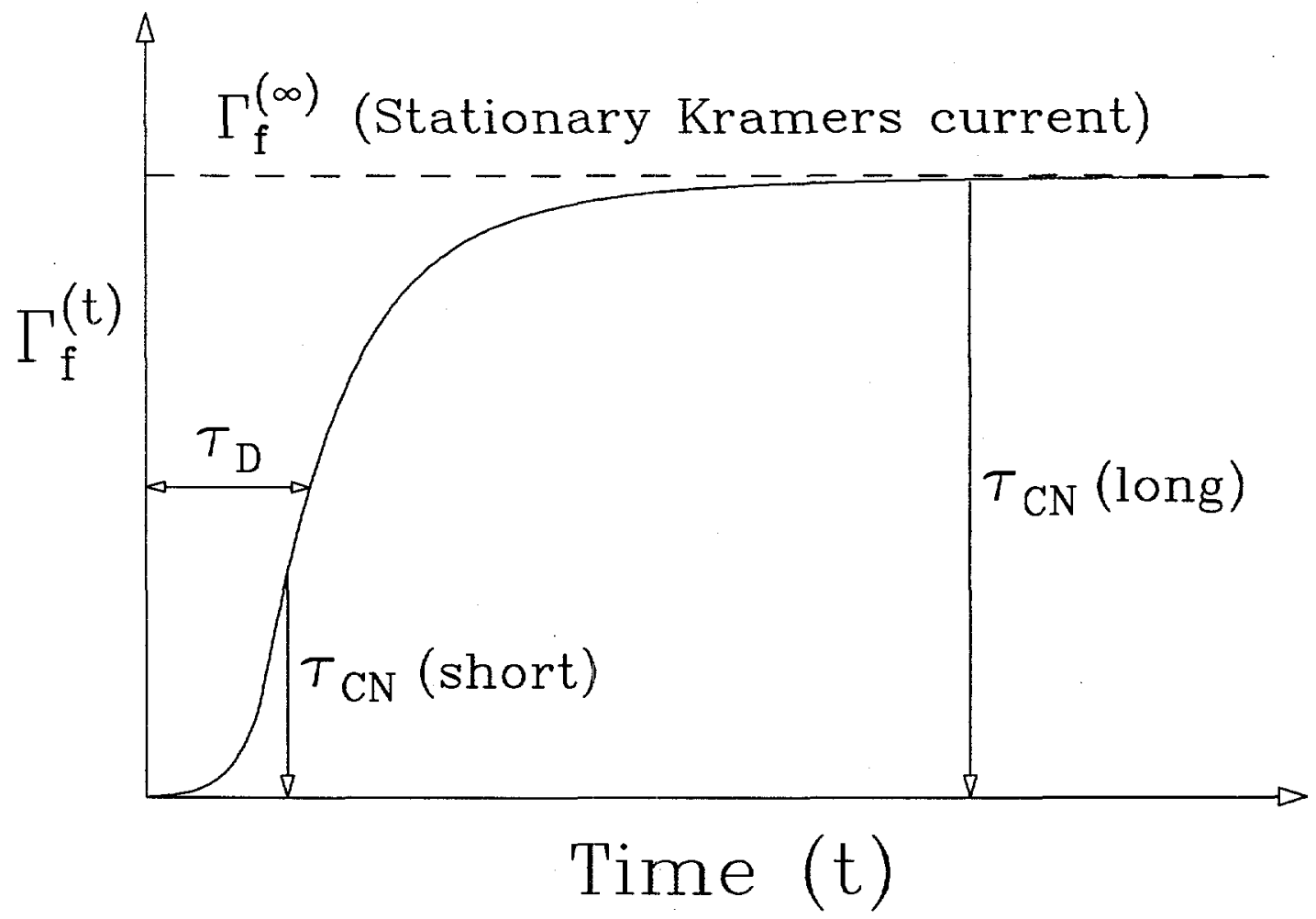

Figure 1.5: A schematic drawing illustrating the transient time effects on the fission decay width.

inferred from the observed prescission particles [Hils 92, Newt 89], although more recent works [Char 97, Mort 97] indicate a shorter transient time.

The fission time scales inferred from prescission particle emssion are the sum of the transient time discussed above and the time required for the nucleus to descend from the saddle to scission, since the prescission particles can be emitted either before the system reaches the fission saddle, or during the descent from the saddle to scission. Postsaddle times do not affect the fission probability. It is therefore necessary to distinguish between presaddle and postsaddle times. Efforts have been made to separate the presaddle and postsaddle time components by examining the differences in the mean kinetic energy of charged particles emitted pre- and post- 
saddle [Lest 93a, Lest 93b, Lest 91]. However, the separation of presaddle and postsaddle particle emission is fraught with difficulties and ambiguities.

Since only the transient time has a strong and direct bearing on the fission probability, the effects of a transient time on the fission probability and the magnitude of the transient time may be determined more reliably from the measured fission probabilities rather than from other means such as particle emission.

In Chapter 4, a new and straight-forward way to determine an upper limit of the transient time, set by the fission probabilities of neighboring isotopes, is presented. This novel approach, which does not involve measuring anything beyond the fission saddle, bypasses all of the difficulties associated with the separation of presaddle and postsaddle particle emission. The new approach is applied to the fission excitation functions of neighboring Os and Po isotopes to extract an upper limit of the fission transient time. The upper limits for transient time determined with this approach are $15 \times 10^{-21}$, and $25 \times 10^{-21} \mathrm{sec}$ for isotopes of Os and Po, respectively. This is in apparent contradiction with a long fission time $\left(\sim 10^{-19} \mathrm{sec}\right)$ claimed in the literature [Hils 92, Newt 89] to explain the measured amounts of prescission neutrons or $\gamma$-rays in relatively heavy fissioning systems. The apparent contradiction may be reconciled when the distinction is made between the presaddle and the postsaddle times. That is, most, if not all, of the fission times determined from light particle emission is postsaddle. 


\subsection{First Chance Fission Probabilities}

The scaling law presented in Section 1.3 is predicted by the transition state formalism for first chance fission probabilities, yet it is well observed by the total fission probabilities. This puzzling aspect of our observed empirical linear scaling has created much concern and anticipation regarding how first chance probabilities should behave. Based on calculations with a particular statistical code, the argument has been made that first chance fission may still be substantially supressed by the effects of a long transient time $\left(10^{-19} \mathrm{sec}\right)$ but the transient time effects are somehow made up with fission probabilities for second and higher chance fission [Back 97]. However, the effects of $\ddot{a}$ transient time as small as $30 \times 10^{-21} \mathrm{sec}$ on the fission probabilities of ${ }^{210} \mathrm{Po}$ are so tremendous that the above argument could not possibly be accommodated [More $97 \mathrm{a}]$. This is especially so in the case of the mass-asymmetric fission of nuclei in the intermediate mass region such as ${ }^{90,94,98} \mathrm{Mo}$, where first chance fission truly dominates throughout the excitation energy range up to $140 \mathrm{MeV}$. Due to the large conditional fission barriers $\left(B_{Z} \sim 40 \mathrm{MeV}\right.$ ), the fission excitation functions are very steep [Jing 99, More 95a]. The fissioning system can not afford losing energy through particle evaporations, since to lose energy before reaching the fission saddle by emitting a particle would greatly decrease the fission probability. First-chance fission, therefore, must dominate.

For heavy fissioning systems ( $\mathrm{Pb}$ or heavier), however, the fission barriers are lower, and multichance fission may become pronounced at high energies. The 
dominance of first chance fission is thus called into question. It is therefore of great interest to determine first chance fission probabilities empirically. Their very sensitivity to the transient time effects would also allow us to set more stringent constraints on the possible transient times. It is, however, very difficult, and even subjective, to sort out the first, second, and higher chances in the total fission probabilities.

The difficulty comes with the uncertainties in the measured total fission probabilities $P_{f}$, which are usually determined as the ratio of the fission cross section to the compound nucleus formation cross section. Although the fission cross sections can be measured very precisely, the formation cross sections are often estimated with models such as the Bass Model [Bass 74], the Extra-push Model [Swia 82], the Optical Model [Macf 78], etc., or just with the geometrical cross section. The estimated fusion cross sections are rather uncertain, and are generally overestimated at high energies, the very energy regime where the dominance of first chance fission is questioned and where the relative contribution of first chance to the total fission probability is to be determined. An overestimation of the fusion cross sections would cause an underestimation in the total fission probabilities, and consequently, lead to an underestimation of the first chance fission probabilities.

Natowitz et al. has used an old recipe to extract the first chance fission probability by comparing the total fission probabilities of neighboring isotopes [Nato 90]. The subjective aspect of the recipe is rooted in its hidden assumption that either the decay clock be reset at each step on the decay chain or the fission transient time be otherwise zero, and therefore the second plus higher chance fission probability 
may be equated to the total fission probability of the neighboring nucleus with one neutron less. This will be further discussed in Chapter 4.

\subsection{Goals of the Project}

In this thesis, a novel approach will be developed to determine upper limits of the fission transient time, which is set by the fission probabilities of neighboring isotopes. The approach will be applied to neighboring Os and Po isotopes to determine the upper limits of their fission transient times.

For this purpose, the fission excitation functions for ${ }^{185,186,187,189} \mathrm{Os}$ produced in the ${ }^{3} \mathrm{He}+{ }^{182,183,184,184} \mathrm{~W}$ reactions, and the fission excitation functions for ${ }^{209,210,211,212} \mathrm{Po}$ produced in the ${ }^{3} \mathrm{He}$ and ${ }^{4} \mathrm{He}+{ }^{206,207,208} \mathrm{~Pb}$ reactions, were measured with high precision. The details of the experiment and the measured fission excitation functions are given in Chapter 2.

These fission excitation functions are analyzed in detail according to the statistical formalism presented in Section 1.2. The fission barriers, and shell effects for the corresponding nuclei are extracted from the detailed analyses. 


\section{Chapter 2}

\section{Measurement of Fission Excitation}

\section{Functions for Os and Po Isotopes}

In this chapter, the fission excitation functions of the compound nuclei ${ }^{185,186,187,189} \mathrm{Os}$ and ${ }^{209,210,211,212} \mathrm{Po}$, produced in the reactions ${ }^{3} \mathrm{He}+{ }^{182,183,184,186, \text { nat }} \mathrm{W}$,

${ }^{3} \mathrm{He}+{ }^{206,207,208, \text { nat }} \mathrm{Pb}$, and ${ }^{4} \mathrm{He}+{ }^{206,207,208, \text { nat }} \mathrm{Pb}$, are presented. These fission excitation functions were measured in experiments carried out at the 88-Inch Cyclotron of the Lawrence Berkeley National Laboratory. Through analysis of these data, one can determine some of the nuclear properties such as the fission barriers, the shell effects, etc., for the fissioning nuclei or their daughters produced by neutron emission. The high precision of the measurements also allows us to make detailed comparisons of the fission probabilities of these neighboring isotopes, and to set upper limits for the dynamic transient time of the fission process. 


\subsection{Experiment}

A variety of compound nuclei (Os and Po isotopes) was prepared by the ${ }^{3} \mathrm{He}$ and ${ }^{4} \mathrm{He}$ induced reactions on targets of $\mathrm{W}$ and $\mathrm{Pb}$ isotopes. ${ }^{3} \mathrm{He}$ beams of 28 different energies between $21 \mathrm{MeV}$ and $140 \mathrm{MeV}$, and ${ }^{4} \mathrm{He}$ beams of 24 different energies between 32 and $118 \mathrm{MeV}$, were used to bombard the targets made from oxides of tungsten isotopes and made from metallic lead isotopes. The uncertainty in beam energies was about $0.3 \%$. The thicknesses and isotopic enrichments of the targets used in the experiments are listed in Table 2.1.

Table 2.1: The thicknesses and isotopic purities of the targets.

\begin{tabular}{lcc}
\hline \hline Target & Thickness $\left(\mu \mathrm{g} / \mathrm{cm}^{2}\right)$ & Purity $(\%)$ \\
\hline${ }^{206} \mathrm{~Pb}$ & $555 \pm 10$ & 97.2 \\
${ }^{207} \mathrm{~Pb}$ & $560 \pm 10$ & 92.0 \\
${ }^{208} \mathrm{~Pb}$ & $500 \pm 10$ & 99.3 \\
${ }^{n a t} \mathrm{~Pb}$ & $544 \pm 10$ & 100 \\
${ }^{182} \mathrm{WO}_{3}$ & $330 \pm 7$ & 94.0 \\
${ }^{183} \mathrm{WO}_{3}$ & $355 \pm 8$ & 82.5 \\
${ }^{184} \mathrm{WO}_{3}$ & $341 \pm 7$ & 93.8 \\
${ }^{186} \mathrm{WO}_{3}$ & $385 \pm 8$ & 97.3 \\
${ }^{\text {nat }} \mathrm{WO}_{3}$ & $363 \pm 8$ & 100 \\
\hline \hline
\end{tabular}

Two large area Parallel Plate Avalanche Counters (PPACs), with an active area of $200 \times 240 \mathrm{~mm}^{2}$ each, were used to detect the fission fragment pairs. Shown in Fig. 2.1 is a photograph of the experiment setup. The two PPACs were placed $15 \mathrm{~cm}$ from the target, and were mounted at $60^{\circ}$ and $240^{\circ}$ with respect to the beam axis, allowing for the detection of both fission fragments in coincidence. The selfsurporting targets ( $20 \mathrm{~mm}$ in diameter) were mounted on a target ladder. The target 
was selected by moving the ladder up or down via remote control. The beams, which were focused on the target, entered the reaction chamber through the hole near the top of the picture.

The PPAC's volume is divided by a cathode foil backed with $2 \mu \mathrm{m}$ thick mylar foil. The cathode was operated at a voltage of $-450 \mathrm{~V}$. The readout of the cathode gives an amplitude and a timing signal, which is independent of position. Two signal wireplanes, one horizontal on one side of the cathode and one vertical on the other side, are mounted at a distance of $3 \mathrm{~mm}$ from the cathode. The $20 \mu \mathrm{m}$ thick wires are separated by a distance of $1 \mathrm{~mm}$. Groups of five wires are combined by a delay line, the readout of which provides the position of the fission fragment. An intrinsic position resolution of $3 \mathrm{~mm}$ vertically and $5 \mathrm{~mm}$ horizontally, allows for the measurement of the folding angle of a fission fragment pair. Fission events were characterized by a prompt relative time peak, large cathode amplitudes, and a back-to-back emission geometry in the center of mass.

Each PPAC has an entrance window made of a stretched polypropylene foil $\left(\sim 100 \mu \mathrm{g} / \mathrm{cm}^{2}\right)$ which separates the isobutane gas in the detector from the chamber vacuum. The PPACs were operated at a constant pressure of 3 torr of isobutane gas.

With lead targets, beam intensities varied from $1 \mathrm{nA}$ at the highest enerigies to $30 \mathrm{nA}$ at the lowest energies. The highest beam intensities were limited to minimize erosion of the lead targets. With tungsten targets, which were in the form of oxides, the beam intensities varied from $10 \mathrm{nA}$ to $350 \mathrm{nA}$. For these targets, the highest beam intensities were limited by the brittleness and the poor thermal 


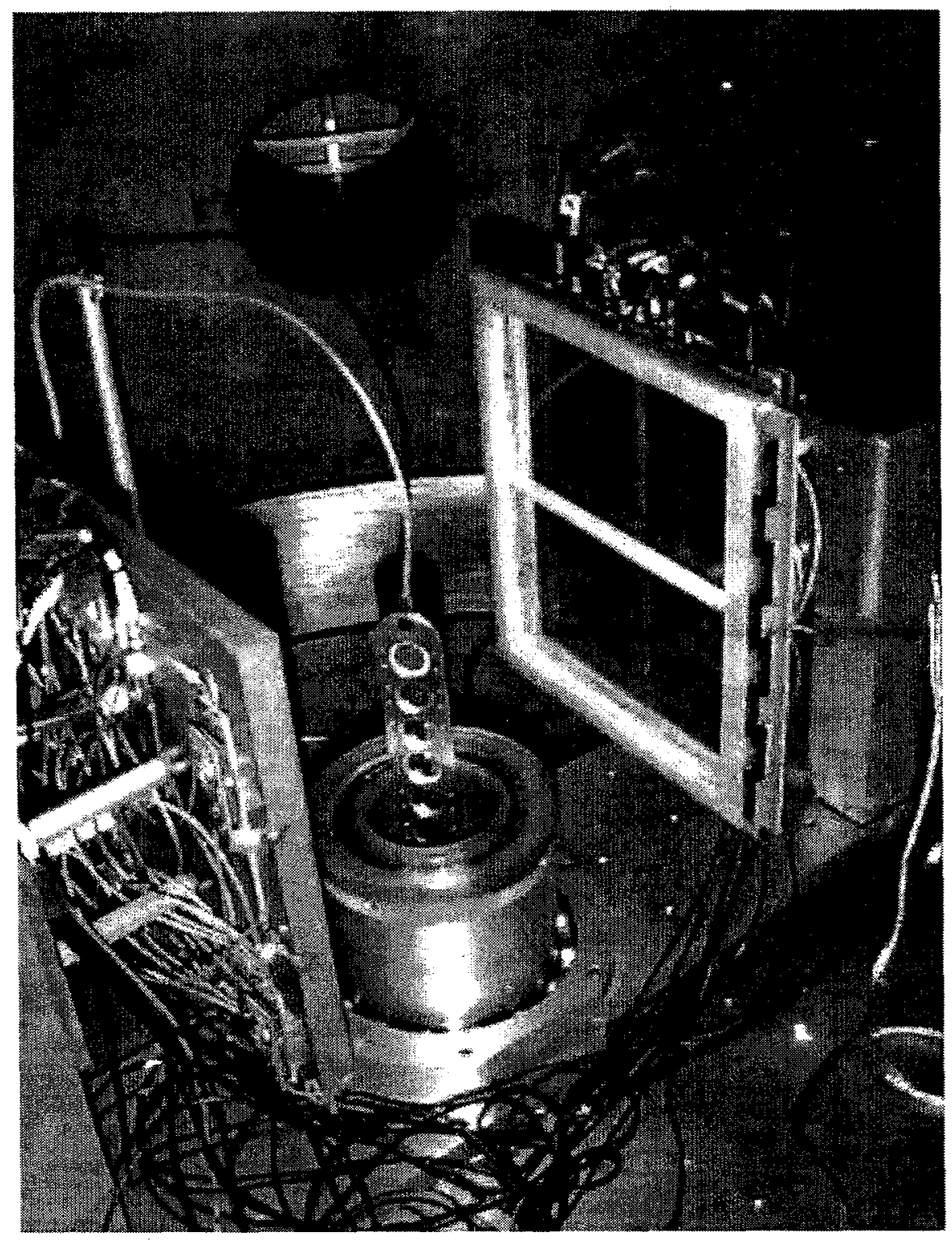

Figure 2.1: A photograph of the experimental setup. 
conductivity of the oxides.

In order to minimize the time needed for beam tuning when changing the beam species and the energies, both the beam species, ${ }^{3} \mathrm{He}$ and ${ }^{4} \mathrm{He}$, were accelerated at the same magnetic rigidity of the cyclotron. Thus, only the electrostatic elements had to be changed, and a significant decrease in cyclotron tuning time was achieved [Clar 90].

High voltage $(8 \mathrm{kV})$ was applied to the target ladder to suppress delta electrons from entering the PPACs, thus improving their signal/noise ratio.

For increasing beam energy, the velocity of the compound nucleus increases in the laboratory frame, resulting in a decreased folding angle between a fission fragment pair. Since our detectors were mounted at fixed angles, and the detection of both fission fragments in a fission event was required, the acceptance of our detector setup had a weak dependence on the beam energy. In our experiments, the geometric acceptance varied from $14 \%$ to $16 \%$ of $4 \pi$, for beam energies from 140 and $21 \mathrm{MeV}$, respectively.

To minimize systematic errors in our measurements, we required a precise determination of the thickness and homogeneity of our targets, which were isotopically enriched and commercially made [Micr 97]. Rutherford backscattering measurements were thus performed. The thickness of the target foils was determined from the widths of the energy spectra of backscattered ${ }^{4} \mathrm{He}$ ions. To determine the overall homogeneity of the targets, we measured the target thickness at different points on each target foil. The thickness fluctuations within the central $10 \mathrm{~mm}$ were found to 
be small $(1 \%)$. In our experiments, the diameter of the beam spot on the target was approximately $5 \mathrm{~mm}$ and the accuracy of the center focus was $\sim 1 \mathrm{~mm}$. The target thicknesses determined by Rutherford backscattering agree well with the thicknesses determined by weighing [Rube 97].

Contamination from heavier elements is potentially another source of systematic uncertainties, especially at the lowest energies where even a very small quantities of heavy contaminants could contribute substantially to the fission yield, because of their lower fission barriers. Fortunately, with targets isotopically enriched by mass separation, this source of contaminant was practically eliminated. No evidence for heavier contaminants was apparent in our measured fission excitation functions which are presented in the next section. Measurement of particle-induced x-ray emission also confirmed that the contaminant level was negligible [Rube 97].

\subsection{Fission Cross Sections}

Fission cross sections $\sigma_{f}$ were determined by using the expression

$$
\sigma_{f}=\frac{n_{f}}{n_{p} n_{t}} \eta
$$

where $n_{f}$ is the number of the detected fission events; $n_{p}$ is the number of incident ${ }^{3} \mathrm{He} /{ }^{4} \mathrm{He}$ particles; and $n_{t}$ is the target thickness (atoms $/ \mathrm{cm}^{2}$ ). The quantity $\eta$ is the inverse of the efficiency of detecting the fission fragment pairs, and is determined by both the geometrical acceptance of the PPACs and the angular distribution of fission fragments. $\eta$ depends not only on the angles of both the PPACs, due to 
anisotropic angular distribution of the fission fragments, but also depends weakly on the beam energy, due to the decreasing folding angle between the pair of fission fragments with increasing beam energy. The angular distribution $\left(\frac{d \sigma}{d \Omega}\right)_{\theta} /\left(\frac{d \sigma}{d \Omega}\right)_{90^{\circ}}$ of the fission fragments was taken to be $1 / \sin \theta$ [Vand 73$]$ in our determination of the factor $\eta$. This should be reasonable, since the deviation of the angular distribution from $1 / \sin \theta$ for non-zero projection of angular momentum on the symmetry axis occurs mostly at angles near $0^{\circ}$ or $180^{\circ}$, where the geometrical weighting factor $d \Omega$ $=\sin \theta d \theta d \phi$ is very small.

The experimental fission excitation functions measured in this work for eight different compound nuclei formed in ten different reactions, are shown in Figs. $2.2 \&$ 2.3. These excitation functions are also tabulated in Tables $2.2,2.3$, and 2.4). The errors given both in the figures and the tables are statistical errors only. The statistical errors are smaller than $1 \%$ for the Po compound nuclei at excitation energies above $40 \mathrm{MeV}$, and the statistical errors are smaller than $2 \%$ for the Os compound nuclei at excitation energies above $50 \mathrm{MeV}$, as can be seen in the Figs. $2.2 \& 2.3$. Systematic errors arise mainly from the uncertainties in the target thicknesses, the beam current integration, and the uncertainties associated with the PPAC acceptance. Overall, the systematic error, which is essentially the same for all reactions forming the neighboring compound nuclei, except for the uncertainties in the target thicknesses, is estimated to be $\sim 4 \%$. The excitation energy was calculated assuming a full momentum and mass transfer of the helium ions to the compound nucleus.

Our measurements of the fission cross sections for several different isotopes 


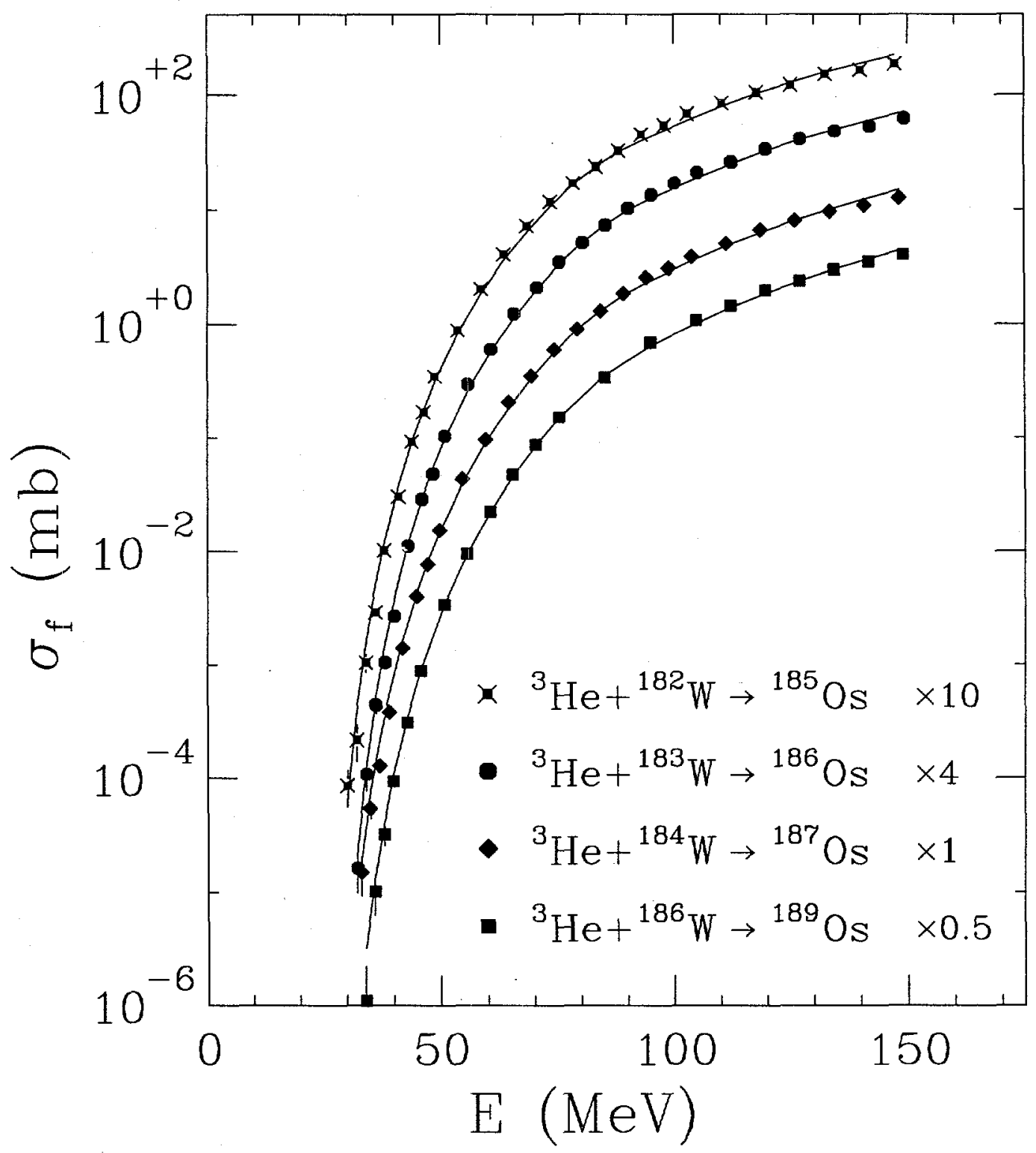

Figure 2.2: The fission excitation functions for osmium isotopes ${ }^{185,186,187,189} \mathrm{O}$ s produced in the reactions ${ }^{3} \mathrm{He}+{ }^{182,183,184,186} \mathrm{~W}$. The lines are to guide the eyes. The statistical errors are shown when they exceed the size of the symbols. The number to the right of each legend indicates the factor by which the corresponding excitation function was multiplied, in order to separate it from its neighboring excitation functions for visual clarity. 


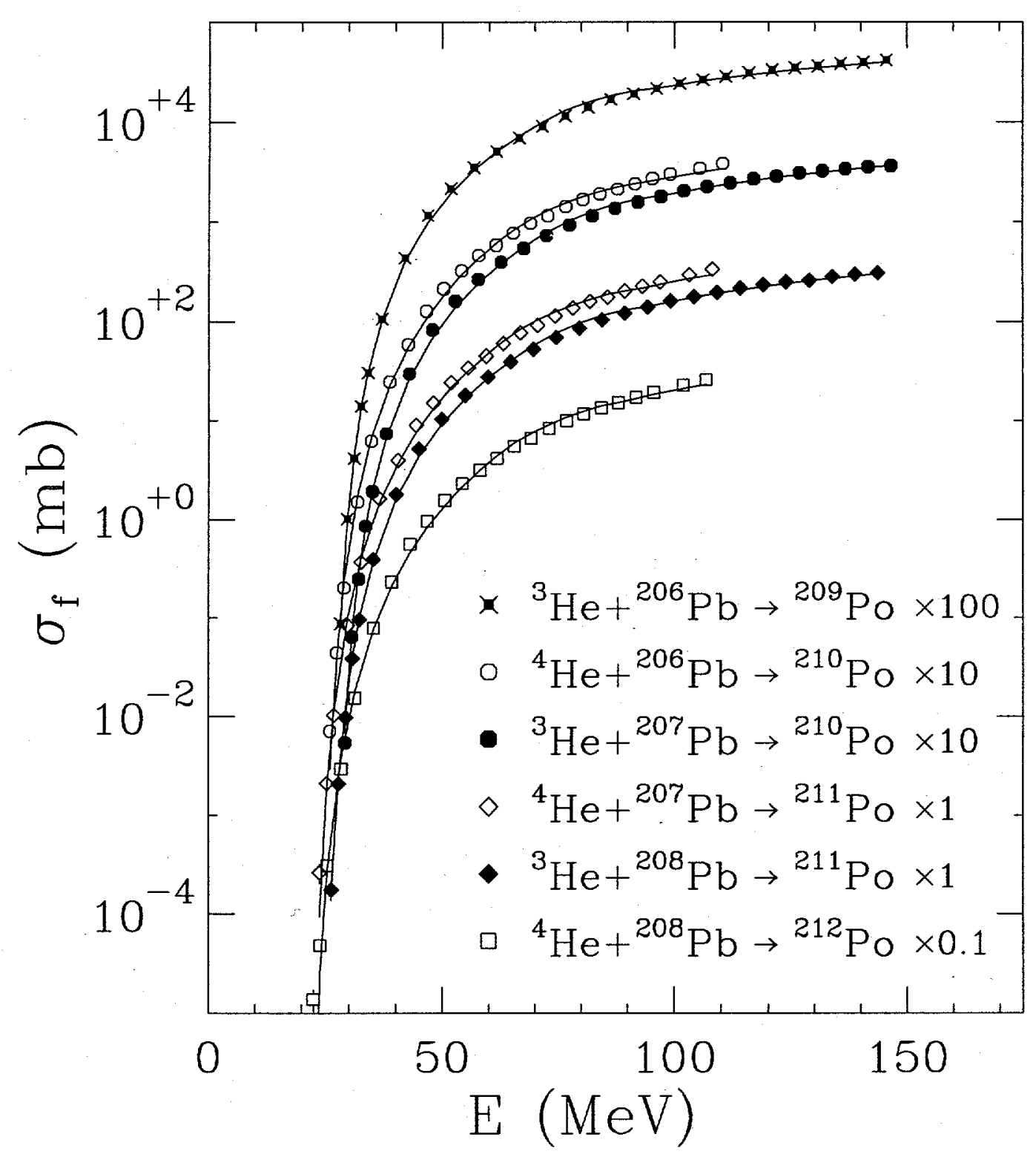

Figure 2.3: Same as Fig. 2.2 for the fission excitation functions of the polonium isotopes $209,210,211,212 \mathrm{Po}$ produced in the reactions ${ }^{3} \mathrm{He}$ (full symbols) and ${ }^{4} \mathrm{He}$ (open symbols) + $206,207,208 \mathrm{~Pb}$. 
Table 2.2: The measured fission cross sections $\sigma_{f}$ of the compound nuclei ${ }^{185,186,187,189} \mathrm{Os}$ and nat $\mathrm{Os}$ produced in ${ }^{3} \mathrm{He}+{ }^{182,183,184,186} \mathrm{~W} \&{ }^{\text {nat }} \mathrm{W}$ reactions. The errors given in the table include the statistical error only.

\begin{tabular}{|c|c|c|c|c|c|}
\hline \multirow{2}{*}{$\begin{array}{c}E_{\text {beam }} \\
(\mathrm{MeV})\end{array}$} & \multicolumn{5}{|c|}{$\sigma_{f}(\mathrm{mb})$} \\
\hline & ${ }^{185} \mathrm{Os}$ & ${ }^{186} \mathrm{Os}$ & ${ }^{187} \mathrm{Os}$ & ${ }^{189} \mathrm{Os}_{\mathrm{s}}$ & ${ }^{\text {nat }} \mathrm{Os}_{\mathrm{s}}$ \\
\hline 21.0 & $(8.6 \pm 3.1) 10^{-6}$ & $(4.1 \pm 1.6) 10^{-6}$ & & & $(6.0 \pm 3.5) 10^{-6}$ \\
\hline 23.0 & $(2.2 \pm 0.8) 10^{-5}$ & $(2.7 \pm 0.8) 10^{-5}$ & $(1.5 \pm 0.6) 10^{-5}$ & $(2.2 \pm 2.2) 10^{-6}$ & $(2.0 \pm 0.6) 10^{-5}$ \\
\hline 25.0 & $(1.0 \pm 0.2) 10^{-4}$ & $(1.1 \pm 0.2) 10^{-4}$ & $(5.4 \pm 1.0) 10^{-5}$ & $(2.0 \pm 0.8) 10^{-5}$ & $(1.0 \pm 0.2) 10^{-4}$ \\
\hline 27.0 & $(2.9 \pm 0.3) 10^{-4}$ & $(2.6 \pm 0.3) 10^{-4}$ & $(1.3 \pm 0.2) 10^{-4}$ & $(6.3 \pm 1.0) 10^{-4}$ & $(2.2 \pm 0.3) 10^{-4}$ \\
\hline 29.0 & $(1.0 \pm 0.1) 10^{-3}$ & $(6.7 \pm 0.5) 10^{-4}$ & $(3.8 \pm 0.4) 10^{-4}$ & $(1.9 \pm 0.2) 10^{-4}$ & $(5.4 \pm 0.4) 10^{-4}$ \\
\hline 32.0 & $(3.0 \pm 0.1) 10^{-3}$ & $(2.8 \pm 0.1) 10^{-3}$ & $(1.4 \pm 0.1) 10^{-3}$ & $(6.2 \pm 0.5) 10^{-4}$ & $(1.9 \pm 0.1) 10^{-3}$ \\
\hline 35.0 & $(9.2 \pm 0.3) 10^{-3}$ & $(7.2 \pm 0.2) 10^{-3}$ & $(4.0 \pm 0.2) 10^{-3}$ & $(1.8 \pm 0.1) 10^{-3}$ & $(5.0 \pm 0.2) 10^{-3}$ \\
\hline 37.5 & $(1.7 \pm 0.2) 10^{-2}$ & $(1.2 \pm 0.1) 10^{-2}$ & $(7.6 \pm 0.6) 10^{-3}$ & & $(9.7 \pm 1.8) 10^{-3}$ \\
\hline 40.0 & $(3.5 \pm 0.1) 10^{-2}$ & $(2.6 \pm 0.1) 10^{-2}$ & $(1.5 \pm .04) 10^{-2}$ & $(6.8 \pm 0.2) 10^{-3}$ & $(2.0 \pm .06) 10^{-2}$ \\
\hline 45.0 & $(8.9 \pm 0.2) 10^{-2}$ & $(7.3 \pm 0.2) 10^{-2}$ & $(4.3 \pm 0.1) 10^{-2}$ & $(1.9 \pm .05) 10^{-2}$ & $(5.4 \pm 0.1) 10^{-2}$ \\
\hline 50.0 & $0.204 \pm 0.004$ & $0.151 \pm 0.004$ & $(9.7 \pm 0.3) 10^{-2}$ & $(4.5 \pm 0.1) 10^{-2}$ & $0.115 \pm 0.003$ \\
\hline 55.0 & $0.407 \pm 0.008$ & $0.311 \pm 0.006$ & $0.206 \pm 0.004$ & $(9.5 \pm 0.3) 10^{-2}$ & $0.244 \pm 0.005$ \\
\hline 60.0 & $0.725 \pm 0.013$ & $0.525 \pm 0.009$ & $0.352 \pm 0.007$ & $0.174 \pm 0.004$ & $0.401 \pm 0.008$ \\
\hline 65.0 & $1.17 \pm 0.02$ & $0.880 \pm 0.018$ & $0.605 \pm 0.006$ & $0.306 \pm 0.006$ & $0.684 \pm 0.014$ \\
\hline 70.0 & $1.69 \pm 0.02$ & $1.29 \pm 0.02$ & $0.901 \pm 0.012$ & & $1.05 \pm 0.01$ \\
\hline 75.0 & $2.38 \pm 0.03$ & $1.83 \pm 0.03$ & $1.30 \pm 0.02$ & $0.678 \pm 0.010$ & $1.51 \pm 0.02$ \\
\hline 80.0 & $3.26 \pm 0.03$ & $2.58 \pm 0.03$ & $1.85 \pm 0.02$ & & $2.10 \pm 0.03$ \\
\hline 85.0 & $4.51 \pm 0.05$ & $3.36 \pm 0.05$ & $2.56 \pm 0.02$ & $1.38 \pm 0.02$ & $2.84 \pm 0.03$ \\
\hline 90.0 & $5.41 \pm 0.05$ & $4.24 \pm 0.04$ & $3.10 \pm 0.03$ & & $3.43 \pm 0.03$ \\
\hline 95.0 & $6.79 \pm 0.07$ & $5.26 \pm 0.05$ & $3.89 \pm 0.04$ & $2.18 \pm 0.03$ & $4.32 \pm 0.04$ \\
\hline 102.5 & $8.39 \pm 0.10$ & $6.51 \pm 0.08$ & $5.07 \pm 0.07$ & $2.92 \pm 0.04$ & $5.35 \pm 0.07$ \\
\hline 110.0 & $10.39 \pm 0.10$ & $8.40 \pm 0.08$ & $6.66 \pm 0.06$ & $3.95 \pm 0.04$ & $6.97 \pm 0.07$ \\
\hline 117.5 & $12.08 \pm 0.17$ & $10.27 \pm 0.14$ & $8.01 \pm 0.12$ & $4.78 \pm 0.08$ & $8.50 \pm 0.12$ \\
\hline 125.0 & $14.91 \pm 0.15$ & $12.02 \pm 0.12$ & $9.60 \pm 0.10$ & $5.99 \pm 0.06$ & $10.12 \pm 0.10$ \\
\hline 132.5 & $16.28 \pm 0.20$ & $13.26 \pm 0.17$ & $10.88 \pm 0.16$ & $7.06 \pm 0.12$ & $11.19 \pm 0.15$ \\
\hline 140.0 & $18.46 \pm 0.18$ & $15.49 \pm 0.15$ & $12.60 \pm 0.13$ & $8.09 \pm 0.08$ & $12.94 \pm 0.13$ \\
\hline
\end{tabular}


Table 2.3: The measured fission cross sections $\sigma_{f}$ of the compound nuclei ${ }^{209,210,211}$ Po and ${ }^{\text {nat }} \mathrm{Po}$ produced in ${ }^{3} \mathrm{He}+{ }^{206,207,208} \mathrm{~Pb} \&{ }^{\text {nat }} \mathrm{Pb}$ reactions. The errors given in the table include the statistical error only.

\begin{tabular}{|c|c|c|c|c|}
\hline \multirow{2}{*}{$\begin{array}{l}E_{\text {beam }} \\
(\mathrm{MeV})\end{array}$} & \multicolumn{4}{|c|}{$\sigma_{f}(\mathrm{mb})$} \\
\hline & ${ }^{209} \mathrm{Po}$ & ${ }^{210} \mathrm{Po}$ & ${ }^{211} \mathrm{Po}$ & ${ }^{\text {nat }} \mathrm{Po}$ \\
\hline 21.0 & $(8.7 \pm 0.9) 10^{-4}$ & $(5.4 \pm 0.6) 10^{-4}$ & $(1.8 \pm 0.4) 10^{-4}$ & $(5.6 \pm 0.9) 10^{-4}$ \\
\hline 22.5 & $(1.0 \pm .07) 10^{-2}$ & $(6.4 \pm 0.5) 10^{-3}$ & $(2.1 \pm 0.2) 10^{-3}$ & $(5.9 \pm 0.4) 10^{-3}$ \\
\hline 24.0 & $(4.2 \pm 0.1) 10^{-2}$ & $(2.5 \pm 0.1) 10^{-2}$ & $(9.8 \pm 0.5) 10^{-3}$ & $(2.2 \pm 0.1) 10^{-2}$ \\
\hline 25.5 & $0.141 \pm 0.004$ & $(8.6 \pm 0.3) 10^{-2}$ & $(3.9 \pm 0.1) 10^{-2}$ & $(8.0 \pm 0.3) 10^{-2}$ \\
\hline 27.0 & $0.307 \pm 0.006$ & $0.194 \pm 0.004$ & $(9.6 \pm 0.2) 10^{-2}$ & $0.188 \pm 0.004$ \\
\hline 30.0 & $1.06 \pm 0.01$ & $0.741 \pm 0.011$ & $0.393 \pm 0.007$ & $0.719 \pm 0.011$ \\
\hline 35.0 & $4.32 \pm 0.04$ & $2.99 \pm 0.03$ & $1.80 \pm 0.02$ & $2.87 \pm 0.03$ \\
\hline 40.0 & $11.6 \pm 0.1$ & $8.20 \pm 0.08$ & $5.19 \pm 0.05$ & $7.89 \pm 0.08$ \\
\hline 45.0 & $21.7 \pm 0.2$ & $16.0 \pm 0.2$ & $10.4 \pm 0.1$ & $15.4 \pm 0.2$ \\
\hline 50.0 & $35.1 \pm 0.3$ & $26.4 \pm 0.2$ & $18.1 \pm 0.2$ & $25.4 \pm 0.2$ \\
\hline 55.0 & $50.8 \pm 0.4$ & $39.3 \pm 0.3$ & $27.5 \pm 0.3$ & $37.7 \pm 0.3$ \\
\hline 60.0 & $69.4 \pm 0.5$ & $53.9 \pm 0.4$ & $39.7 \pm 0.3$ & $52.8 \pm 0.4$ \\
\hline 65.0 & $91.0 \pm 0.6$ & $72.7 \pm 0.5$ & $52.5 \pm 0.4$ & $69.4 \pm 0.5$ \\
\hline 70.0 & $115.9 \pm 0.8$ & $93.4 \pm 0.8$ & $68.7 \pm 0.7$ & $88.9 \pm 0.8$ \\
\hline 75.0 & $141.6 \pm 0.9$ & $115.7 \pm 0.8$ & $85.2 \pm 0.8$ & $110.4 \pm 0.8$ \\
\hline 80.0 & $168.7 \pm 1.2$ & $137.4 \pm 1.1$ & $104.3 \pm 1.0$ & $132.5 \pm 1.1$ \\
\hline 85.0 & $192.5 \pm 1.3$ & $158.4 \pm 1.1$ & $121.0 \pm 1.1$ & $152.3 \pm 1.1$ \\
\hline 90.0 & $216.7 \pm 1.3$ & $180.9 \pm 1.2$ & $139.0 \pm 1.1$ & $176.0 \pm 1.2$ \\
\hline 95.0 & $244.0 \pm 1.8$ & $205.1 \pm 1.6$ & $161.3 \pm 1.5$ & $197.8 \pm 1.6$ \\
\hline 100.0 & $266.2 \pm 1.8$ & $226.5 \pm 1.7$ & $176.6 \pm 1.6$ & $216.3 \pm 1.7$ \\
\hline 105.0 & $286.7 \pm 1.9$ & $247.9 \pm 1.8$ & $196.2 \pm 1.7$ & $237.7 \pm 1.7$ \\
\hline 110.0 & $311.1 \pm 2.0$ & $270.5 \pm 1.8$ & $214.4 \pm 1.7$ & $260.9 \pm 1.8$ \\
\hline 115.0 & $332.7 \pm 2.0$ & $286.9 \pm 1.9$ & $231.7 \pm 1.8$ & $281.0 \pm 1.9$ \\
\hline 120.0 & $350.1 \pm 2.1$ & $309.7 \pm 2.0$ & $249.6 \pm 1.9$ & $295.0 \pm 2.0$ \\
\hline 125.0 & $363.4 \pm 2.2$ & $323.6 \pm 2.0$ & $256.8 \pm 1.9$ & $310.2 \pm 2.0$ \\
\hline 130.0 & $384.3 \pm 1.8$ & $338.8 \pm 1.7$ & $280.7 \pm 1.6$ & $330.0 \pm 1.7$ \\
\hline 135.0 & $397.0 \pm 2.3$ & $355.0 \pm 2.1$ & $294.0 \pm 2.1$ & $345.4 \pm 2.1$ \\
\hline 140.0 & $416.0 \pm 2.3$ & $363.2 \pm 2.2$ & $304.1 \pm 2.1$ & $352.1 \pm 2.1$ \\
\hline
\end{tabular}


Table 2.4: The measured fission cross sections $\sigma_{f}$ of the compound nuclei ${ }^{210,211,212}$ Po and ${ }^{\text {nat }} \mathrm{Po}$ produced in ${ }^{4} \mathrm{He}+{ }^{206,207,208} \mathrm{~Pb} \&{ }^{\text {nat }} \mathrm{Pb}$ reactions. The errors given in the table include the statistical error only.

\begin{tabular}{c|cccc}
\hline \hline \multirow{2}{*}{$E_{\text {beam }}$} & \multicolumn{4}{c}{$\sigma_{f}(\mathrm{mb})$} \\
\cline { 2 - 5 }$(\mathrm{MeV})$ & ${ }^{210} \mathrm{Po}$ & ${ }^{211} \mathrm{Po}$ & ${ }^{212} \mathrm{Po}$ & ${ }^{\text {nat }} \mathrm{Po}$ \\
\hline 32.0 & $(7.1 \pm 0.8) 10^{-4}$ & $(2.6 \pm 0.6) 10^{-4}$ & $(1.3 \pm 0.3) 10^{-4}$ & $(6.5 \pm 0.8) 10^{-4}$ \\
33.5 & $(4.4 \pm 0.2) 10^{-3}$ & $(2.1 \pm 0.1) 10^{-3}$ & $(4.8 \pm 0.6) 10^{-4}$ & $(2.6 \pm 0.2) 10^{-3}$ \\
35.0 & $(2.0 \pm 0.1) 10^{-2}$ & $(1.0 \pm .05) 10^{-2}$ & $(3.1 \pm 0.3) 10^{-3}$ & $(1.1 \pm .06) 10^{-2}$ \\
38.0 & $0.151 \pm 0.004$ & $(8.4 \pm 0.2) 10^{-2}$ & $(3.0 \pm 0.1) 10^{-2}$ & $(8.7 \pm 0.2) 10^{-2}$ \\
41.0 & $0.624 \pm 0.010$ & $0.368 \pm 0.006$ & $0.155 \pm 0.003$ & $0.344 \pm 0.006$ \\
45.0 & $2.45 \pm 0.026$ & $1.63 \pm 0.02$ & $0.786 \pm 0.011$ & $1.49 \pm 0.02$ \\
49.0 & $5.83 \pm 0.059$ & $3.97 \pm 0.04$ & $2.32 \pm 0.03$ & $3.82 \pm 0.04$ \\
53.0 & $12.7 \pm 0.1$ & $8.99 \pm 0.08$ & $5.63 \pm 0.06$ & $8.66 \pm 0.09$ \\
56.8 & $21.2 \pm 0.2$ & $15.2 \pm 0.2$ & $9.66 \pm 0.10$ & $14.4 \pm 0.2$ \\
60.7 & $32.1 \pm 0.3$ & $24.1 \pm 0.2$ & $15.7 \pm 0.2$ & $23.0 \pm 0.2$ \\
64.4 & $45.8 \pm 0.3$ & $34.2 \pm 0.3$ & $23.2 \pm 0.3$ & $32.8 \pm 0.3$ \\
68.3 & $58.5 \pm 0.4$ & $45.0 \pm 0.3$ & $31.6 \pm 0.3$ & $44.0 \pm 0.3$ \\
72.1 & $77.0 \pm 0.5$ & $59.6 \pm 0.4$ & $41.7 \pm 0.4$ & $57.4 \pm 0.4$ \\
75.9 & $97.6 \pm 0.7$ & $76.5 \pm 0.6$ & $55.2 \pm 0.6$ & $74.5 \pm 0.6$ \\
79.7 & $115.7 \pm 0.9$ & $91.8 \pm 0.8$ & $66.9 \pm 0.7$ & $89.8 \pm 0.8$ \\
83.6 & $143.3 \pm 1.3$ & $113.6 \pm 1.0$ & $83.9 \pm 0.8$ & $110.2 \pm 1.0$ \\
87.4 & $168.5 \pm 1.5$ & $136.7 \pm 1.3$ & $99.7 \pm 1.0$ & $128.7 \pm 1.2$ \\
91.2 & $191.7 \pm 1.3$ & $158.9 \pm 1.1$ & $117.8 \pm 1.0$ & $151.0 \pm 1.2$ \\
95.1 & $214.0 \pm 1.6$ & $175.3 \pm 1.5$ & $135.0 \pm 1.4$ & $171.4 \pm 1.5$ \\
98.9 & $241.7 \pm 1.8$ & $200.9 \pm 1.5$ & $153.0 \pm 1.2$ & $191.7 \pm 1.6$ \\
102.7 & $273.9 \pm 1.9$ & $226.1 \pm 1.7$ & $171.8 \pm 1.6$ & $219.0 \pm 1.7$ \\
106.6 & $302.5 \pm 2.0$ & $248.2 \pm 1.8$ & $194.4 \pm 1.7$ & $238.8 \pm 1.8$ \\
113.0 & $344.7 \pm 2.1$ & $290.9 \pm 1.9$ & $228.8 \pm 1.8$ & $280.5 \pm 1.9$ \\
118.0 & $384.6 \pm 2.3$ & $333.4 \pm 2.1$ & $259.8 \pm 1.9$ & $319.9 \pm 2.1$ \\
\hline \hline & & & &
\end{tabular}


of the same element, and for a target of that element containing isotopes of known abundances (natural, for example), allow us to examine the self-consistency of the measurements. Shown in Fig. 2.4 are the ratios of the helium-induced fission cross section of nat $\mathrm{Pb}$, as synthesized with the fission cross sections measured for the individual lead isotopes, to the helium-induced fission cross section for nat $\mathrm{Pb}$, as measured using a natural lead target. In synthesizing the helium-induced fission cross section of ${ }^{\text {nat }} \mathrm{Pb}$ from the helium-induced fission cross sections of the individual $\mathrm{Pb}$ isotopes, the cross section for ${ }^{204} \mathrm{~Pb}$, which was not measured in our experiments, was estimated from the cross sections for the ${ }^{208} \mathrm{~Pb}$ and ${ }^{206} \mathrm{~Pb}$ by extrapolation. This estimation should be sufficient for the purpose of synthesizing the overall cross section, since the abundance of ${ }^{204} \mathrm{~Pb}$ in natural lead is only $1.42 \%$. The fact that the ratios are equal to unity within $3 \%$ over the entire energy range, verifies the high precision of our measurements. This ratio is very sensitive to isotopic purities of the targets, particularly the isotopic purity of ${ }^{208} \mathrm{~Pb}$ target, at low energies where the fission cross sections decreases substantially with increasing neutron numbers (see Tables 2.3 and 2.4). Thus the observed ratios close to unity also verified that our ${ }^{208} \mathrm{~Pb}$ target was indeed isotopically very pure.

The same consistency was also verified for the measured ${ }^{3} \mathrm{He}$-induced fission cross sections of tungsten isotopes (see Fig. 2.5).

For a given compound nucleus $\left({ }^{210} \mathrm{Po}\right.$ or $\left.{ }^{211} \mathrm{Po}\right)$, the ${ }^{4} \mathrm{He}-\mathrm{ind}$ uced fission cross section at a given excitation energy is higher than the ${ }^{3} \mathrm{He}$-induced fission cross section at the same excitation energy, as can be seen in Fig. 2.3. This is mainly 


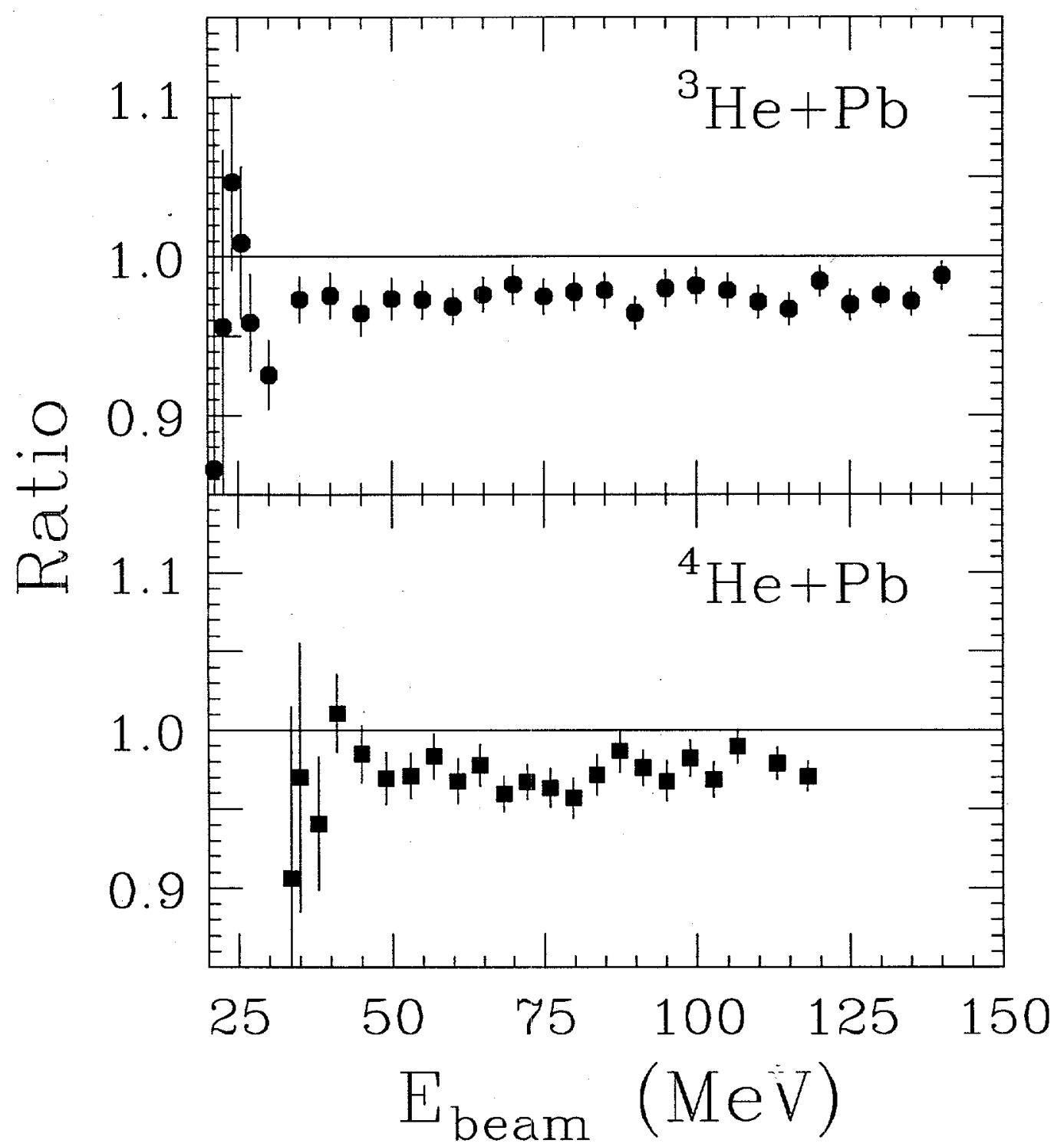

Figure 2.4: The ratios of the ${ }^{3} \mathrm{He} /{ }^{4} \mathrm{He}$-induced fission cross sections of natural lead, as calculated from the fission cross sections measured for the individual lead isotopes, and the ${ }^{3} \mathrm{He} /{ }^{4} \mathrm{He}$-induced fission cross sections of natural lead, as measured using a natural lead target. Shown in the upper panel is the result for the ${ }^{3} \mathrm{He}$-induced fission of lead $\left({ }^{3} \mathrm{He}+\right.$ nat,206,207,208 $\mathrm{Pb}$ ); and shown in the lower panel is the result for the ${ }^{4} \mathrm{He}$-induced fission of lead $\left({ }^{4} \mathrm{H}\left[\mathrm{e}+{ }^{\text {nat, } 206,207,208} \mathrm{~Pb}\right)\right.$. 


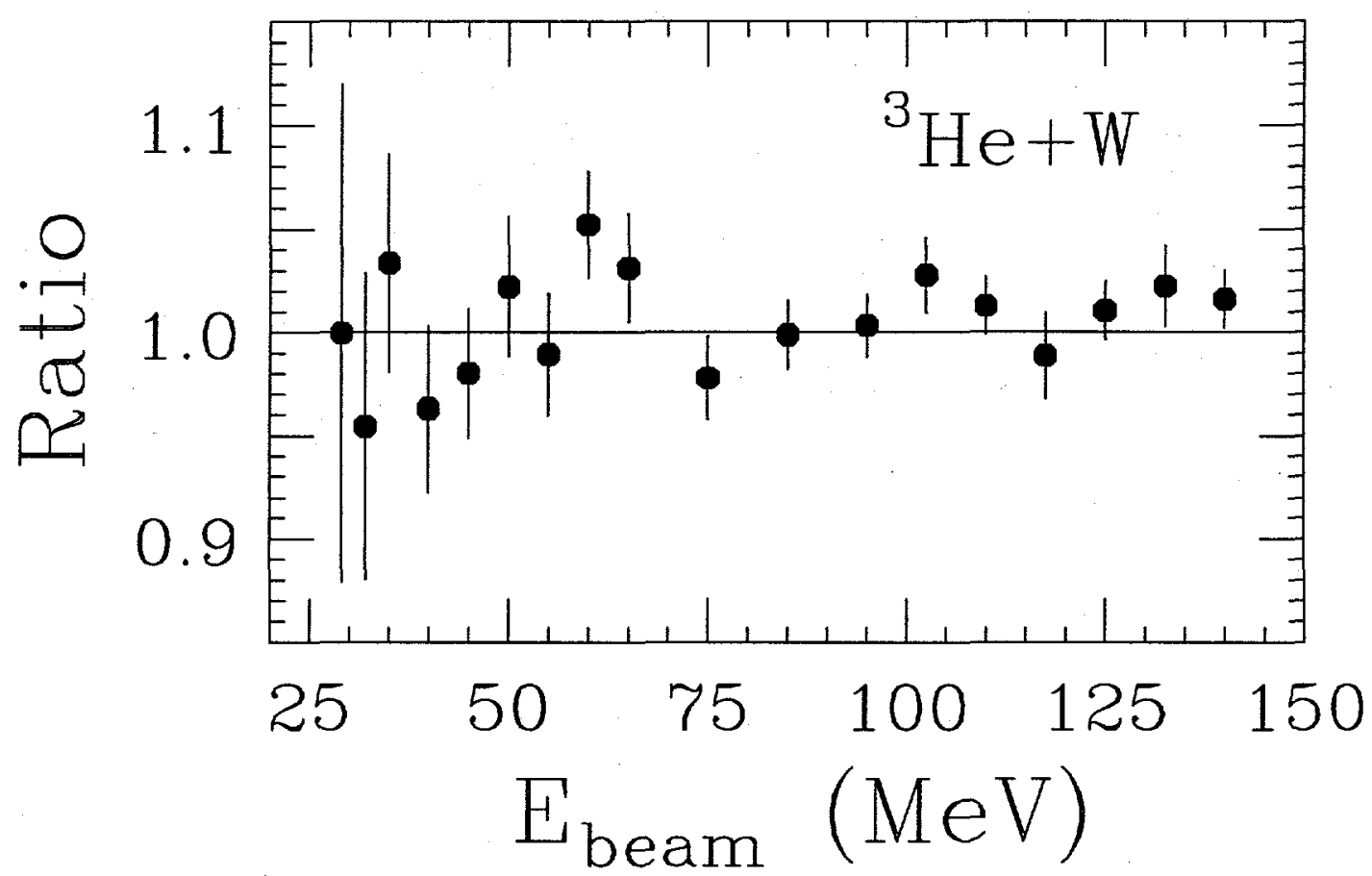

Figure 2.5: The ratios of the ${ }^{3} \mathrm{He}$-induced fission cross sections of natural tungsten, as calculated from the fission cross sections measured for the individual tungsten isotopes $\left({ }^{3} \mathrm{He}+{ }^{182,183,184,186} \mathrm{~W}\right)$, and the ${ }^{3} \mathrm{He}$-induced fission cross sections of natural tungsten, as measured using a natural tungsten target $\left({ }^{3} \mathrm{He}+{ }^{\text {nat }} \mathrm{W}\right)$.

due to the higher fusion cross sections of the ${ }^{4} \mathrm{He}$-induced reactions (see Figs. 3.2 and 3.3 in next chapter). The difference in the angular momentum of the compound nuclei formed by different entrance channels also plays a role, resulting in different branching ratios for fission due to differences in rotational energies. However, since the angular momenta of the compound nuclei formed in ${ }^{3} \mathrm{He} /{ }^{4} \mathrm{He}$-induced reactions are generally small, the angular momentum effects on the fission probabilities are not expected to be significant.

In an earlier experiment [Rube 96] we measured the fission excitation functions for the compound nuclei ${ }^{200} \mathrm{Tl},{ }^{211} \mathrm{Po}$, and ${ }^{212} \mathrm{At}$ formed in the ${ }^{3} \mathrm{He}$-induced 
reactions on ${ }^{197} \mathrm{Au},{ }^{208} \mathrm{~Pb}$, and ${ }^{209} \mathrm{Bi}$, respectively. The data from these measurements are in excellent agreement with our current ones.

Even earlier, during the period 1960-1970, the fission excitation functions of ${ }^{208,210,211,212} \mathrm{Po}$ produced in ${ }^{4} \mathrm{He}$-induced reactions on ${ }^{204,206,207,208} \mathrm{~Pb}$, and of other nuclei, were measured over the excitation energy range from $120 \mathrm{MeV}$ down to an energy very close to the fission barriers [Khod 66]. These earlier data deviate from the current measurements by up to $20 \%$ depending on the targets. The discrepancies are not well understood. However, the deviations are not significant. 


\section{Chapter 3}

\section{Fission Barriers and Shell Effects}

The fission excitation functions presented in Chapter 2 can be interpreted and analyzed according to the statistical formalism illustrated in Chapter 1.2. Several physical parameters, such as fission barriers, shell effects, etc., of the corresponding nuclei are contained in the fission excitation functions, and can therefore be determined from detailed analyses of these excitation functions. The standard analysis procedure is to fit the measured fission excitation functions with the statistical formalism, using the fission barriers, shell effects, etc., as free parameters in the fits.

In this chapter, the measured fission excitation functions are analyzed and fitted with the statistical formalism, under various assumptions for the compound nucleus formation cross sections. The fission barriers and shell effects, etc., extracted from these fits are presented and discussed in the following. 


\subsection{Formation Cross Sections of the Fissioning Nuclei}

The fission probability of a compound nucleus can be calculated with the statistical formalism. However, to calculate the fission cross section, one needs to know the formation cross section of the fissioning compound nucleus (see Eq. 1.20). In. the ${ }^{3} \mathrm{He} /{ }^{4} \mathrm{He}-$ induced fission, the formation cross section of the fissioning compound nucleus is the fusion cross section of the projectile and the target nucleus. The fusion process occurs over a range of impact parameters from head-on collision to the maximum impact parameter. The compound nucleus is, therefore, formed with a distribution of angular momenta. The maximum impact parameter, and thus the maximum angular momentum $\ell_{\max }$, can be related directly to the total fusion cross section. Assuming a sharp spin cut-off at the maximum angular momentum $\ell_{\max }$, the angular momentum distribution of the fusion cross section $\sigma_{\ell}$ becomes

$$
\sigma_{\ell}=\pi \lambda^{2}(2 \ell+1), \quad \ell=0,1,2, \cdots, \ell_{\max },
$$

where $\lambda$ is the reduced wave length $(\lambda=\hbar / p$ with $p$ being the momentum of the incident particle in the center-of-mass frame). The total fusion cross section $\sigma_{0}$ is

$$
\sigma_{0}=\sum_{0}^{\ell_{\max }} \sigma_{\ell}=\pi \lambda^{2} \sum_{0}^{\ell_{\max }}(2 \ell+1)=\pi \star^{2}\left(\ell_{\max }+1\right)^{2} .
$$

The angular momentum distribution of the fusion cross section $\sigma_{\ell}$ can also be parameterized using a Fermi distribution [Plic 83, Hass 85], to take into account a more realistic diffuseness of the distribution near $\ell_{\max }$

$$
\sigma_{\ell}=\frac{\pi \lambda^{2}(2 \ell+1)}{1+\exp \left[\left(\ell-\ell_{\max }\right) / \delta \ell\right]},
$$


where $\delta \ell$ determines the diffuseness of the angular momentum distribution.

The fusion cross sections for these reaction systems are, however, not well known, and have to be estimated with theoretical models such as the Bass model [Bass 74], the Extra-push model [Swia 82], etc..

The Bass model [Bass 74] assumes a frozen shape of the colliding nuclei during their approach, and takes a conservative two-body potential which includes the effects of the finite range of nuclear forces. The Bass model assumes that fusion, or the transition from the entrance channel to the compound nucleus, occurs at the point of contact where the projectile and target densities in the overlap region add up to saturation density of nuclear matter. Energy and angular momentum dissipation by friction are considered in the limit of strong localization at the point of contact.

The Extra-push model [Swia 82] considers the further time evolution of the system (either towards fusion or reseparation) after two nuclei have been brought into contact. The evolution is governed by the relative magnitudes of the repulsive electric forces and the cohesive nuclear forces. For light systems, for which the electric forces are small, the configuration of tangent nuclei is expected to evolve automatically towards fusion. For heavier nuclei, the electric repulsion may become so large that, starting from rest at contact, the system will reseparate. In this case, an additional energy - an extra push - is thus needed to achieve fusion.

The fusion cross sections calculated with the Bass model and the Extrapush model for the reactions ${ }^{3} \mathrm{He}+{ }^{184} \mathrm{~W},{ }^{3} \mathrm{He}+{ }^{207} \mathrm{~Pb}$ and ${ }^{4} \mathrm{He}+{ }^{207} \mathrm{~Pb}$ are shown in Figs. 3.1, 3.2 and 3.3. Also plotted in these figures are the reaction cross sections 


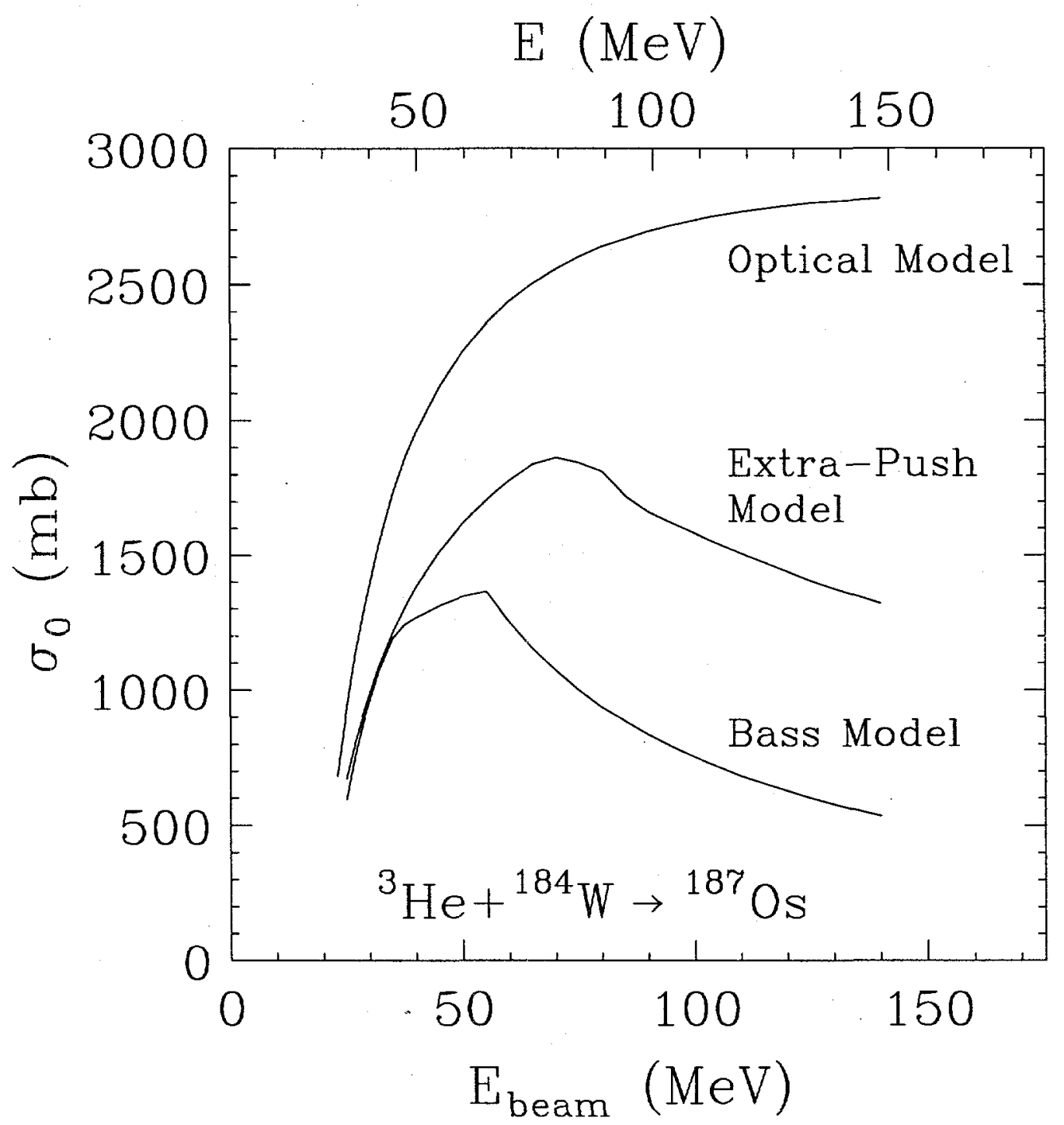

Figure 3.1: The fusion cross sections $\sigma_{0}$ predicted for the reaction ${ }^{3} \mathrm{He}+{ }^{184} \mathrm{~W}$ by the Bass model [Bass 74] and the Extra-push model [Swia 82], plotted as a function of the ${ }^{3} \mathrm{He}$ beam energy $E_{\text {beam. }}$. The excitation energy $E$ of the resulting compound nucleus ${ }^{187} \mathrm{Os}$ is indicated on the top. The reaction cross section calculated with the Optical model [Macf $78]$ is also given. 


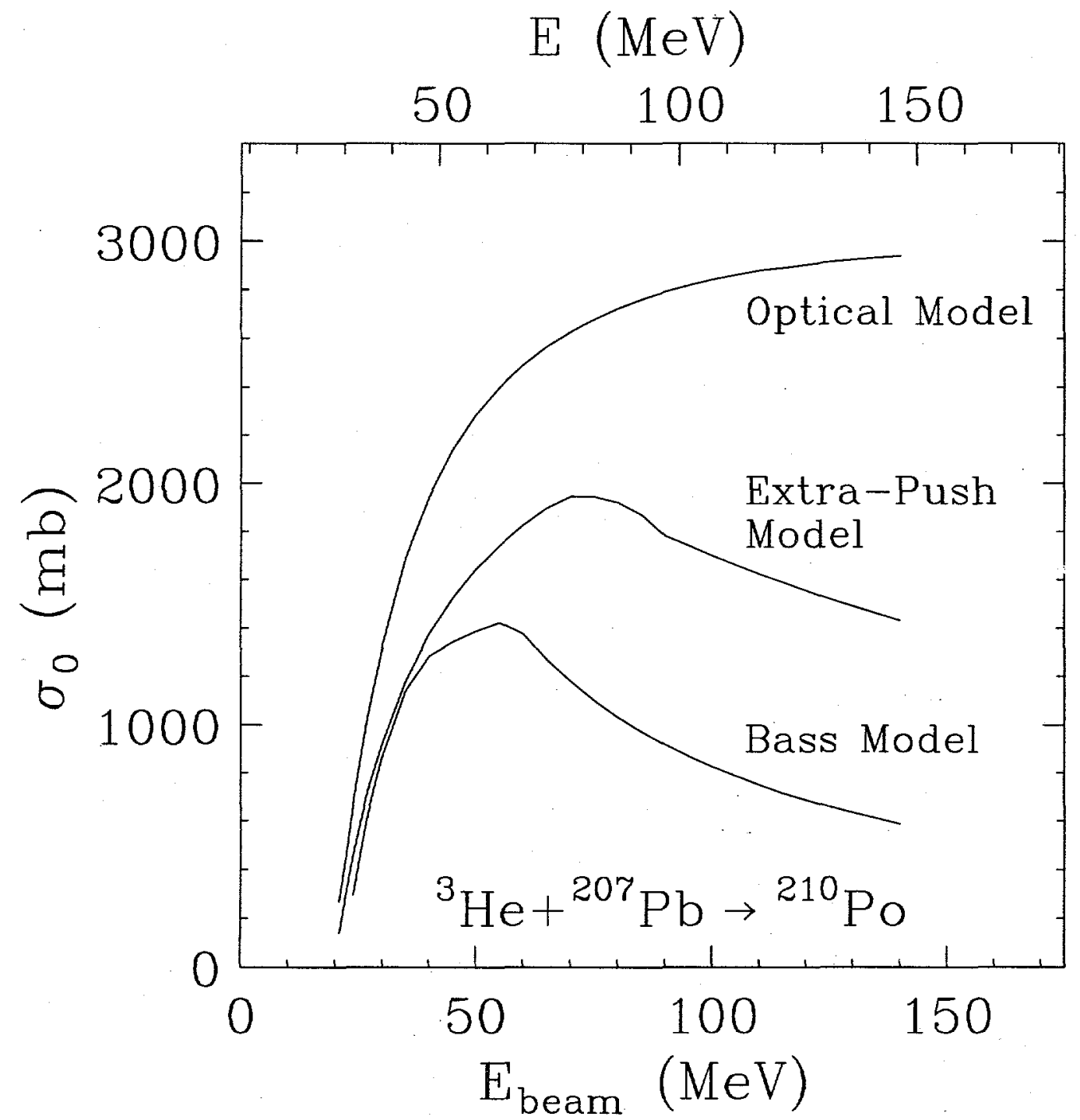

Figure 3.2: The fusion cross sections $\sigma_{0}$ predicted for the reaction ${ }^{3} \mathrm{He}+{ }^{207} \mathrm{~Pb}$ by the Bass model [Bass 74] and the Extra-push model [Swia 82], plotted as a function of the ${ }^{3} \mathrm{He}$ beam energy $E_{\text {beam. }}$. The excitation energy $E$ of the resulting compound nucleus ${ }^{210} \mathrm{Po}_{\mathrm{o}}$ indicated on the top. The reaction cross section calculated with the Optical model [Macf $78]$ is also given. 


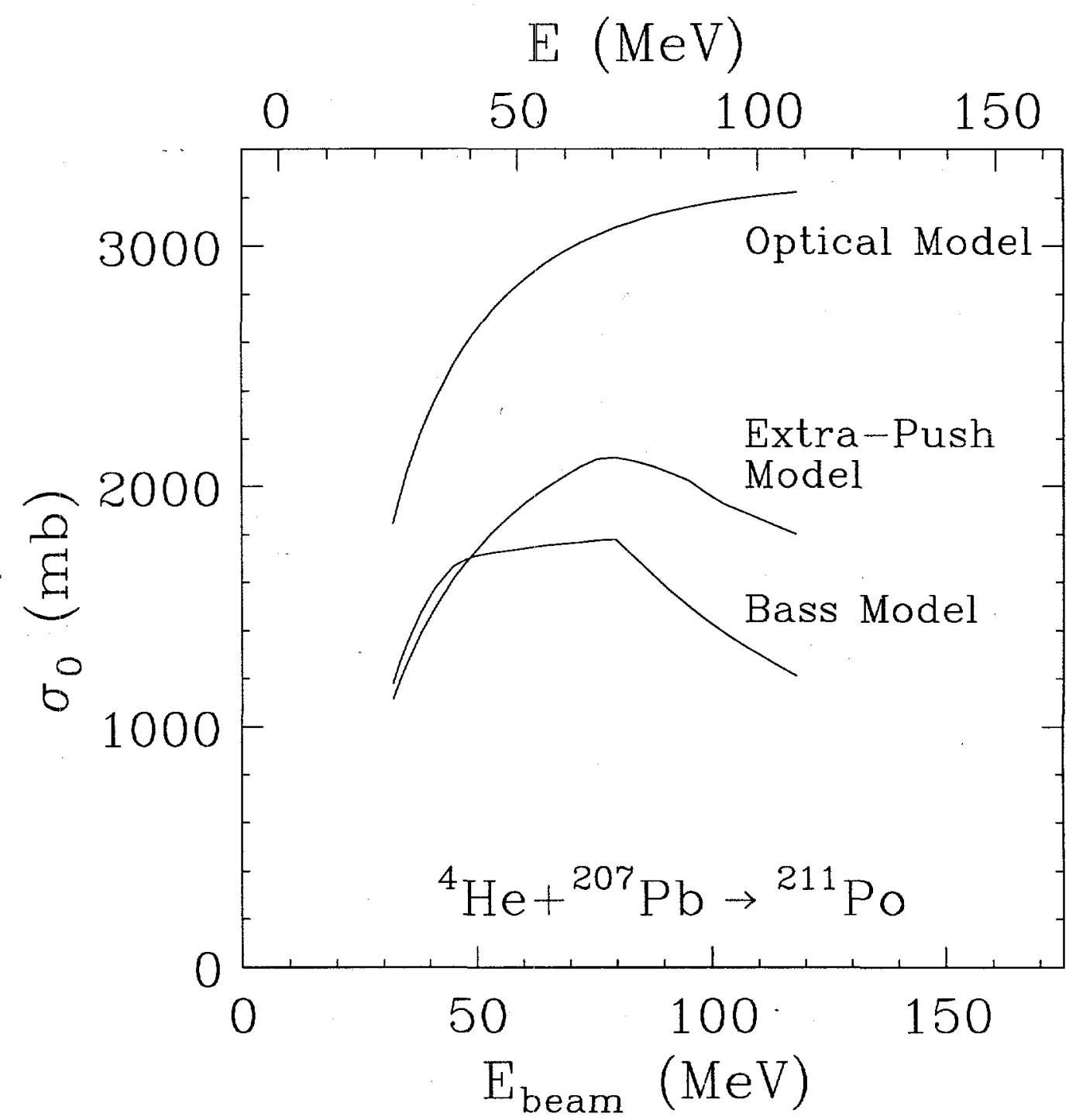

Figure 3.3: The fusion cross sections $\sigma_{0}$ predicted for the reaction ${ }^{4} \mathrm{He}+{ }^{207} \mathrm{~Pb}$ by the Bass model [Bass 74] and the Extra-push model [Swia 82], plotted as a function of the ${ }^{3} \mathrm{He}$ beam energy $E_{\text {beam. }}$. The excitation energy $E$ of the resulting compound nucleus ${ }^{211}$ Po is indicated on the top. The reaction cross section calculated with the Optical model [Macf $78]$ is also given. 
predicted by the Optical model [Fesh 54, Macf 78]. The reaction cross section from the Optical model includes both the fusion cross section and the cross sections for direct reactions, and may thus be considered as an upper limit of the formation cross section of compound nucleus.

Having estimated the fusion cross sections of the fissioning nuclei, we can now calculate the fission cross sections with the statistical formalism. A fit of the calculated fission cross sections as a function of energy to the measured fission excitation function should allow us to determine the effective fission barrier $B_{f}^{*}$, the ratio of the level density parameter at the saddle point to that of the ground state $\left(a_{f} / a_{n}\right)$, and the shell effect of the daughter nucleus after neutron emission $\Delta_{\text {shell }}^{n-1}$, by using these physical quantities as free parameters in the fitting procedure.

As shown above, the uncertainty in the calculated fusion cross sections is quite substantial (see Figs. 3.1, 3.2 and 3.3). Presumably, the exponential dependence of the fission probability on the square root of the internal excitation energy at the saddle point should be so dominant a factor that it may make this uncertainty rather irrelevant, if the fusion cross sections, however uncertain, depend on energy only weakly. However, in the cases where the fusion barriers of the entrance channel reactions are comparable to the fission barriers, such as in the He-induced fission in the lead region, the fusion cross sections may play a larger role in the determination of the fission cross sections. In these cases, some difficulties may be expected due to the uncertainty in the fusion cross sections. To find out what difficulties the uncertainty may cause, and to determine its effects on the extracted parameters, 
we will proceed with our analysis in the following order: 1) fitting the excitation functions with $\Delta_{\text {shell }}^{n-1}$ fixed with its nominal value from ref. [Möll 93], leaving $B_{f}^{*}$ and $a_{f} / a_{n}$ as free parameters; 2) fitting the excitation functions with $B_{f}^{*}$ fixed, leaving $\Delta_{\text {shell }}^{n-1}$ and $a_{f} / a_{n}$ as free parameters; 3 ) fitting the excitation functions with all three as free parameters.

\subsection{Fission Barriers}

As shown in Eqs. 1.9 and 1.10, the angular momentum dependence of $\Gamma_{f}$ and $\Gamma_{n}$ is taken into account by the addition of the rotational energy at the saddle $E_{r}^{s}$ and the energy of the rotating ground state $E_{r}^{g s}$ (which are not available for the internal excitation), to the fission barrier $B_{f}$ and the neutron separation energy $B_{n}$, respectively. The rotational energy of the nucleus at the saddle point is $E_{r}^{s}=$ $\hbar^{2} \ell(\ell+1) / 2 \Im_{\text {saddle }}$ where $\Im_{\text {saddle }}$ is the moment of inertia about the axis perpendicular to the symmetry axis of the nucleus at the saddle point. In this analysis $\Im_{\text {saddle }}$ is calculated assuming a saddle configuration of two spheres separated by $2 \mathrm{fm}$. Since nuclei in the lead region are spherical or very nearly so, the energy of the rotating ground state $E_{r}^{g s}$ is thus equal to the rotational energy of the ground state, $E_{r}^{g s}=$ $\hbar^{2} \ell(\ell+1) / 2 \Im_{\mathrm{gs}}$ where $\Im_{\mathrm{gs}}$ is the moment of inertia of the ground state. In calculating $\Im_{\text {saddle }}$ and $\Im_{\mathrm{gs}}$, the radius of a nuclear sphere is taken to be $R=1.2 A^{1 / 3} \mathrm{fm}$.

The angular momentum $\ell$ of a fissioning nucleus is determined by the product of the projectile momentum and the impact parameter. The maximum angular 
momentum $\ell_{\max }$, or the maximum impact parameter at which a projectile and a target nucleus can be brought to form a compound nucleus, are calculated with three different models - the Bass model [Bass 74], the Extra-push model [Swia 82], and the Optical model [Macf 78]. $\ell_{\max }$ is related to the fusion cross section $\sigma_{0}$ by Eq. 3.3, or by Eq. 3.2 assuming a sharp cut-off at $\ell_{\max }$ in $\ell$ distribution. In this analysis, the diffuseness parameter $\delta \ell$ is taken to be 1 , implying a nearly sharp spin cut-off. The fitting is insensitive to the specific value of $\delta \ell$, since, for $\ell<\ell_{\max }$, variations of $\delta \ell$ from 0 to $\infty$ result only in changes of $\sigma_{\ell}$ by at most a factor of 2 .

The statistical formalism requires the use of a specific level-density expression in the widths $\Gamma_{f}$ and $\Gamma_{n}$. It is mainly in the level densities that all of the physical information concerning the nucleus at the saddle point and the residual nucleus after neutron emission is contained. For the level density, we have used the approximate Fermi gas expression $\rho(E) \propto \exp (2 \sqrt{a E})$, where $E$ is the thermal excitation energy of the system and $a$ is the level density parameter: In the calculation of the fission cross section with Eq. 1.20, the branching ratio for fission $P_{f}(\ell)$ is $\Gamma_{f} /\left(\Gamma_{n}+\Gamma_{f}\right)$, where $\Gamma_{f} / \Gamma_{n}$ can be evaluated with Eq. 1.12. The level densities $\rho\left(E-B_{n}-E_{r}^{g s}\right)$ and $\rho_{s}\left(E-B_{f}-E_{r}^{s}\right)$ can in turn be evaluated with Eqs. 1.14 and 1.17, respectively.

Multichance fission is expected to occur at high energies. To avoid complications from possible multichance fission, we shall, for now, limit ourselves to fit only the low-energy portion of the excitation functions where the fission probability is most sensitive to the fission barrier $B_{f}$ and first chance fission is dominant.

The low-energy portion of the fission excitation functions for the nuclei 
${ }^{185,186,187,189} \mathrm{Os}$ produced in ${ }^{3} \mathrm{He}$-induced reactions on the targets of ${ }^{182,183,184,186} \mathrm{~W}$ (see Table 2.2 and Fig. 2.2), and of the fission excitation functions for the nuclei ${ }^{209,210,211,212} \mathrm{Po}$ produced in ${ }^{3} \mathrm{He} /{ }^{4} \mathrm{He}$-induced reactions on the targets of ${ }^{206,207,208} \mathrm{~Pb}$ (see Table 2.3, 2.4 and Fig. 2.3), were therefore fitted with two free parameters: $B_{f}^{*}$ - the effective fission barrier, and $a_{f} / a_{n}$ - the ratio of the level density parameter at the saddle point to that of the compound nucleus at the ground state. A level density parameter for the compound nucleus $a=a_{n}=A / 8\left(\mathrm{MeV}^{-1}\right)$ was assumed in the fitting. For the residual nucleus after neutron evaporation, $a_{n}=(A-1) / 8$ $\left(\mathrm{MeV}^{-1}\right)$. The nominal value of the shell effect for the residual nucleus after neutron emission $\Delta_{\text {shell }}^{n-1}$, taken to be $E_{\text {mic }}+\left(M_{\text {exp }}-M_{\text {th }}\right)$ from ref. [Möll 93], and listed in Table 3.1, was used as a fixed input parameter. (In ref. [Möll 93], $E_{\text {mic }}$ denotes $\left(M_{\mathrm{th}}\right.$ - $\left.M_{\text {L.D.sph }}\right)$ - the difference between the theoretical mass $M_{\text {th }}$ and the mass of the liquid-drop sphere $M_{\text {L.D.sph. }}$ Thus, $E_{\text {mic }}+\left(M_{\exp }-M_{\mathrm{th}}\right)=\left(M_{\text {exp }}-M_{\text {L.D.sph }}\right)$ where $M_{\text {exp }}$ is the experimental mass. Throughout this thesis, the shell effects $\Delta_{\text {shell }}$ take the opposite sign of those in ref. [Möll 93], i.e., $\Delta_{\text {shell }}=\left(M_{\text {L.D.sph }}-M_{\text {exp }}\right)$.) The energy range of the data points used in the fitting is indicated by $E_{H}$, the highest excitation energy of the data points in the range, which was listed in Table 3.1. $E_{H}$ was chosen to be around $70 \mathrm{MeV}$, below which the sheer steepness of the excitation function implies undoubtedly the dominance of the first chance fission.

Shown in Fig. 3.4 are the fission excitations of the nuclei ${ }^{185,186,187,189} \mathrm{Os}$ produced in the ${ }^{3} \mathrm{He}+{ }^{182,183,184,186} \mathrm{~W}$ reactions, and the corresponding fits with three different assumptions, i.e., the Bass model [Bass 74], the Extra-push model [Swia 82] 
and the Optical model [Macf 78], for the formation cross sections of the fissioning nuclei. These formation cross sections of ${ }^{187} \mathrm{Os}$ from the reaction ${ }^{3} \mathrm{He}+{ }^{184} \mathrm{~W}$ are presented in Fig. 3.1 in Section 3.1. The formation cross sections of ${ }^{185,186,189} \mathrm{Os}$ from the reactions ${ }^{3} \mathrm{He}+{ }^{182,183,186} \mathrm{~W}$ are taken to be the same as those of ${ }^{187} \mathrm{Os}$ from the reaction ${ }^{3} \mathrm{He}+{ }^{184} \mathrm{~W}$ at the same incident energy. In the data range where the fitting was attempted, all the fits are excellent, despite the substantial difference in the formation cross sections predicted by the different models. In Fig. 3.4, all the fitted lines were extended to the highest energy data point, using the parameters $B_{f}^{*}$ and $a_{f} / a_{n}$ extracted from the fits.

The extracted fission barriers $B_{f}^{*}$ and $a_{f} / a_{n}$ are listed in Table 3.1. The extracted barriers are remarkably consistent, and very little affected by the large uncertainties in the formation cross sections predicted by the different models. The fission barriers extracted using the formation cross sections from the different models agree with each other within $0.5 \mathrm{MeV}$. For example, the extracted fission barriers for ${ }^{187} \mathrm{Os}$ are $22.33,21.86$, and $21.99 \mathrm{MeV}$ by using the formation cross sections from the Bass model [Bass 74], the Extra-push model [Swia 82] and the Optical model [Macf $78]$, respectively. For other nuclei $\left({ }^{185} \mathrm{Os},{ }^{186} \mathrm{Os}\right.$ and $\left.{ }^{189} \mathrm{Os}\right)$, one sees the same, or better, agreement between the barriers extracted using different model calculations for the formation cross sections. Thus, given the shell effect of the residual nucleus after neutron emission, one is able to determine the fission barriers $B_{f}^{*}$ to an uncertainty of about $0.5 \mathrm{MeV}$, despite the large uncertainties in the formation cross sections of the fissioning nuclei. 


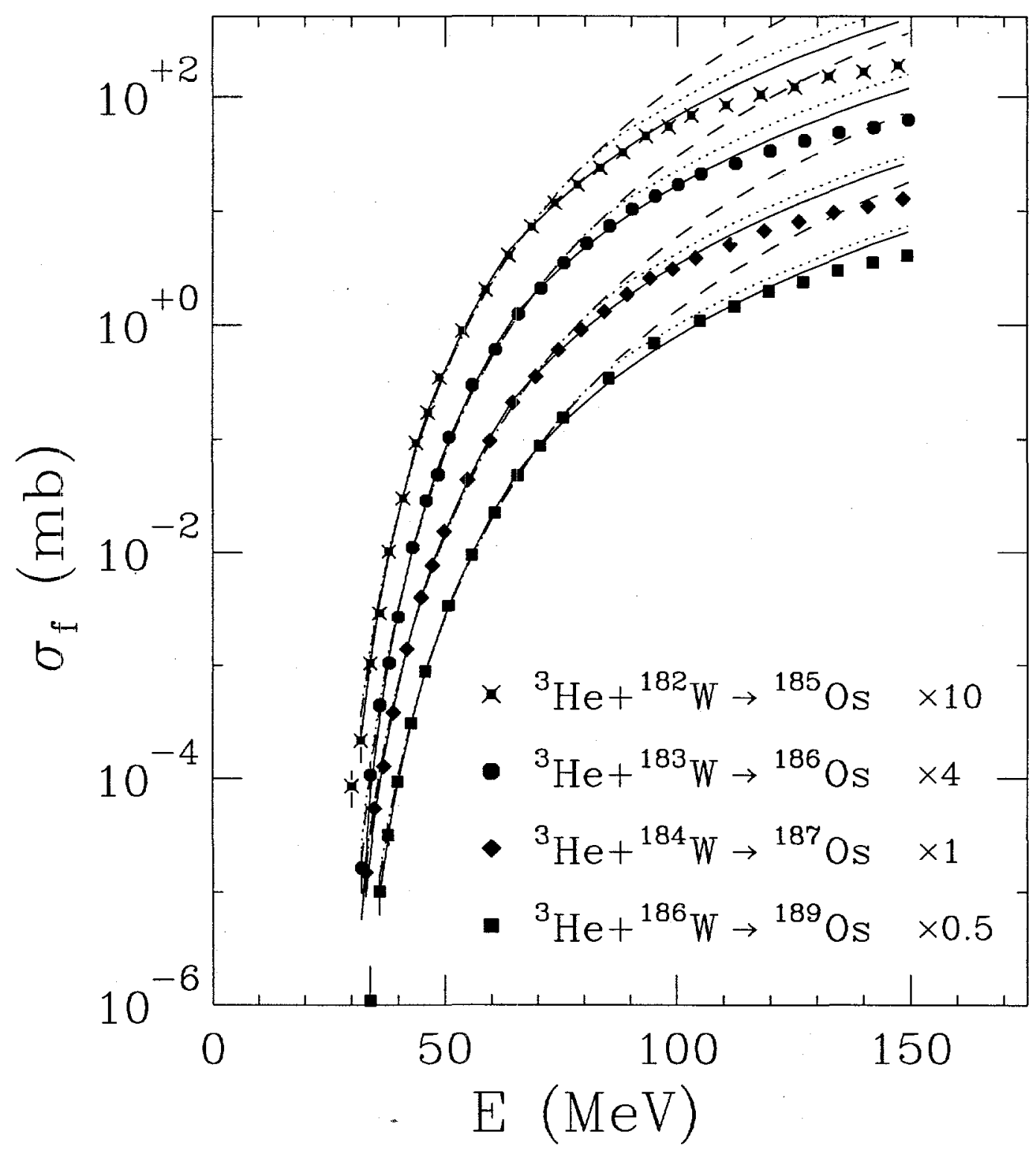

Figure 3.4: The fission excitation functions for the nuclei ${ }^{185,186,187,189} \mathrm{Os}$ produced in the reactions ${ }^{3} \mathrm{He}+{ }^{182,183,184,186} \mathrm{~W}$, were fitted with the statistical formalism, with $B_{f}^{*}$ and $a_{f} / a_{n}$ as two free parameters. The solid lines, the dotted lines and the dashed lines represent fits using the formation cross sections of the fissioning nuclei calculated by the Bass, the Extra-push and the Optical models, respectively. The fits were attempted for only the low energy portion of the fission excitation functions. The highest energy of the data segment used in the fitting is indicated by $E_{H}$ in Table 3.1. The fits were extended to the full range of the experimental data, using the extracted parameters ( $B_{f}^{*}$ and $a_{f} / a_{n}$ ) which are listed in Table 3.1. The number to the right of each legend indicates the factor by which the corresponding excitation function is multiplied for visual clarity. 


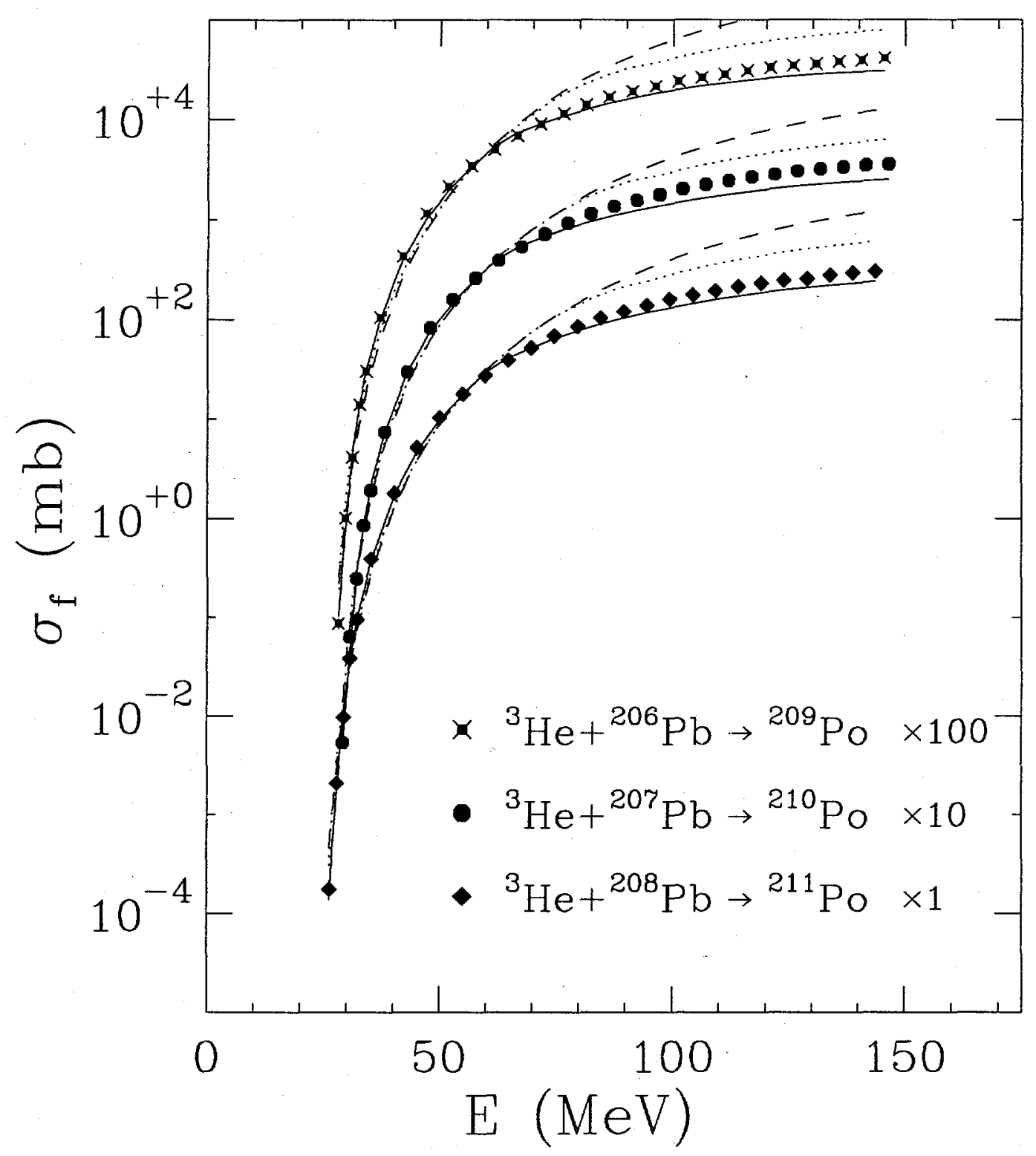

Figure 3.5: The fission excitation functions for the nuclei ${ }^{209,210,211}$ Po produced in the reactions ${ }^{3} \mathrm{He}+{ }^{206,207,208} \mathrm{~Pb}$, were fitted with the statistical formalism, with $B_{f}^{*}$ and $a_{f} / a_{n}$ as two free parameters. The solid lines, the dotted lines and the dashed lines represent fits using the formation cross sections of the fissioning nuclei calculated by the Bass, the Extra-push and the Optical models, respectively. The fits were attempted for only the low energy portion of the fission excitation functions. The highest energy of the data segment used in the fitting is indicated by $E_{H}$ in Table 3.1. The fits were extended to the full range of the experimental data, using the extracted parameters $\left(B_{f}^{*}\right.$ and $\left.a_{f} / a_{n}\right)$ which are listed in Table 3.1. The number to the right of each legend indicates the factor by which the corresponding excitation function is multiplied for visual clarity. 


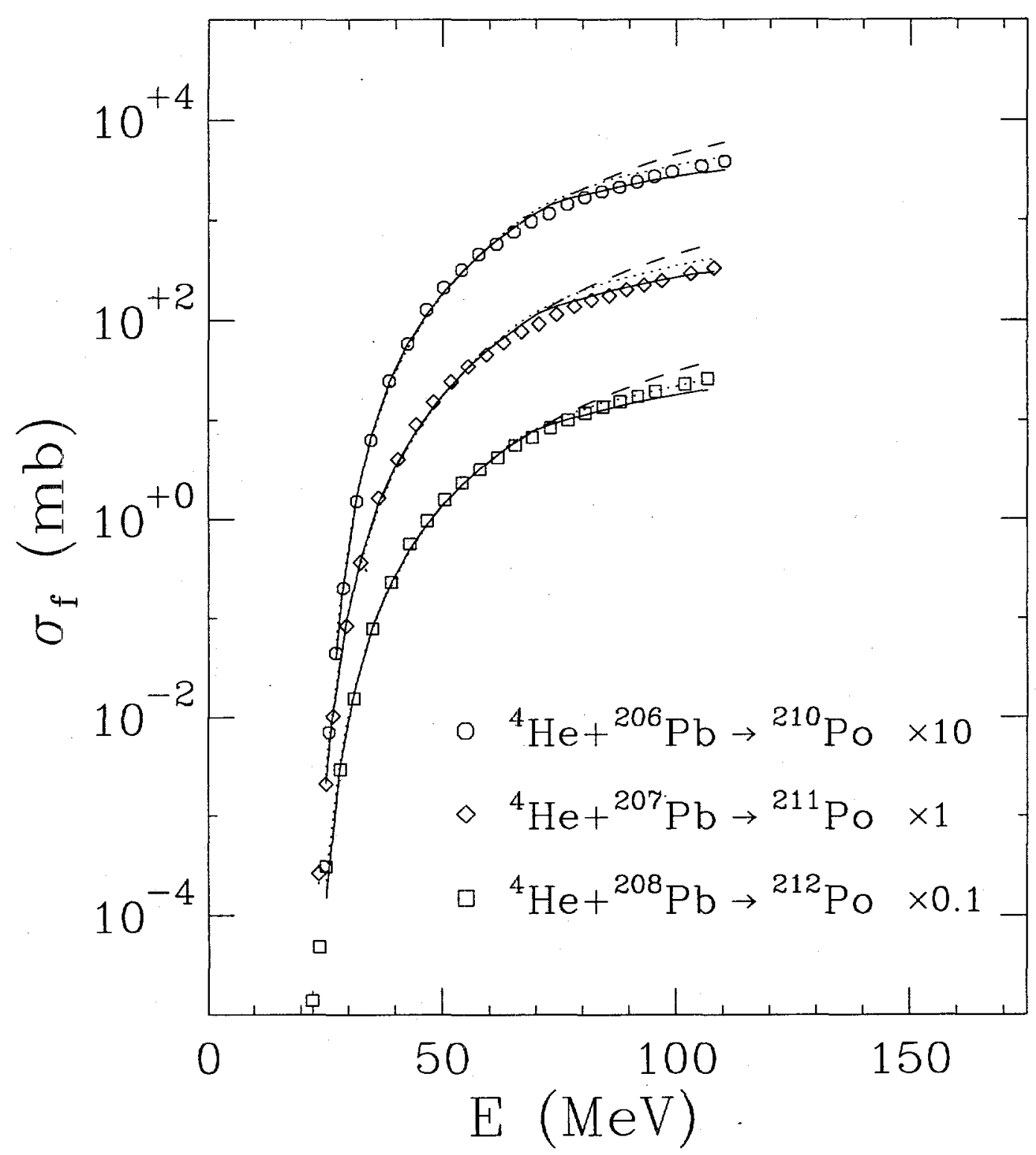

Figure 3.6: The fission excitation functions for the nuclei ${ }^{210,211,212}$ Po produced in the reactions ${ }^{4} \mathrm{He}+{ }^{206,207,208} \mathrm{~Pb}$, were fitted with the statistical formalism, with $B_{f}^{*}$ and $a_{f} / a_{n}$ as two free parameters. The solid lines, the dotted lines and the dashed lines represent fits using the formation cross sections of the fissioning nuclei calculated by the Bass, the Extra-push and the Optical models, respectively. The fits were attempted for only the low energy portion of the fission excitation functions. The highest energy of the data segment used in the fitting is indicated by $E_{H}$ in Table 3.1. The fits were extended to the full range of the experimental data, using the extracted parameters $\left(B_{f}^{*}\right.$ and $\left.a_{f} / a_{n}\right)$ which are listed in Table 3.1. The number to the right of each legend indicates the factor by which the corresponding excitation function is multiplied for visual clarity. 
Table 3.1: The values of $B_{f}^{*}$ and $a_{f} / a_{n}$ extracted by fitting the low energy portion of the measured fission excitation functions. The data and the corresponding fits are shown in Figs. 3.4, 3.5 and 3.6. The fits were performed with three different assumptions for the formation cross sections of the fissioning compound nuclei (CN): the Bass, the Extra-push and the Optical models. $a_{n}=A / 8\left(\mathrm{MeV}^{-1}\right)$ was assumed. $\Delta_{\text {FRDM }}^{n-1}(\mathrm{MeV})$ is the nominal shell effects of the daughter nucleus after neutron emission taken to be $E_{\text {mic }}+\left(M_{\exp }-M_{\mathrm{th}}\right)$ in ref. [Möll 93] and used as a fixed parameter in the fitting. $E_{H}(\mathrm{MeV})$ indicates the highest excitation energy of the data points up to which the fits were attempted.

\begin{tabular}{|c|c|c|c|c|c|c|c|}
\hline Reaction & $\mathrm{CN}$ & $E_{H}$ & $\Delta_{\text {FRDM }}^{n-1}$ & $B_{f}^{*}(\mathrm{MeV})$ & $a_{f} / a_{n}$ & $\chi^{2}$ & $\sigma_{0}$ \\
\hline${ }^{3} \mathrm{He}+{ }^{182} \mathrm{~W}$ & ${ }^{185} \mathrm{Os}$ & 73.4 & 0.85 & $\begin{array}{l}21.43 \pm 0.05 \\
21.05 \pm 0.04 \\
21.17 \pm 0.02\end{array}$ & $\begin{array}{l}1.103 \pm 0.002 \\
1.078 \pm 0.002 \\
1.063 \pm 0.001\end{array}$ & $\begin{array}{r}8.4 \\
39.1 \\
30.2\end{array}$ & $\begin{array}{l}\text { Bass } \\
\text { Extra-push } \\
\text { Optical }\end{array}$ \\
\hline${ }^{3} \mathrm{He}+{ }^{183} \mathrm{~W}$ & ${ }^{186} \mathrm{Os}$ & 75.5 & 1.18 & $\begin{array}{l}23.42 \pm 0.05 \\
23.04 \pm 0.05 \\
23.15 \pm 0.05\end{array}$ & $\begin{array}{l}1.095 \pm 0.002 \\
1.070 \pm 0.002 \\
1.054 \pm 0.002\end{array}$ & $\begin{array}{l}13.5 \\
32.9 \\
24.9\end{array}$ & $\begin{array}{l}\text { Bass } \\
\text { Extra-push } \\
\text { Optical }\end{array}$ \\
\hline${ }^{3} \mathrm{He}+{ }^{184} \mathrm{~W}$ & ${ }^{187} \mathrm{Os}$ & 74.4 & 1.32 & $\begin{array}{l}22.33 \pm 0.05 \\
21.86 \pm 0.05 \\
21.99 \pm 0.05\end{array}$ & $\begin{array}{l}1.098 \pm 0.002 \\
1.070 \pm 0.002 \\
1.056 \pm 0.002\end{array}$ & $\begin{array}{r}6.1 \\
22.9 \\
17.5\end{array}$ & $\begin{array}{l}\text { Bass } \\
\text { Extra-push } \\
\text { Optical }\end{array}$ \\
\hline${ }^{3} \mathrm{He}+{ }^{186} \mathrm{~W}$ & ${ }^{189} \mathrm{Os}$ & 75.4 & 1.87 & $\begin{array}{l}23.23 \pm 0.06 \\
22.72 \pm 0.06 \\
22.86 \pm 0.06\end{array}$ & $\begin{array}{l}1.090 \pm 0.002 \\
1.062 \pm 0.002 \\
1.048 \pm 0.002\end{array}$ & $\begin{array}{l}4.6 \\
6.3 \\
4.1\end{array}$ & $\begin{array}{l}\text { Bass } \\
\text { Extra-push } \\
\text { Optical }\end{array}$ \\
\hline${ }^{3} \mathrm{He}+{ }^{206} \mathrm{~Pb}$ & ${ }^{209} \mathrm{Po}$ & 66.6 & 9.06 & $\begin{array}{l}21.80 \pm 0.02 \\
22.00 \pm 0.01 \\
22.38 \pm 0.02\end{array}$ & $\begin{array}{l}1.086 \pm 0.001 \\
1.082 \pm 0.001 \\
1.075 \pm 0.001\end{array}$ & $\begin{array}{r}24.3 \\
209.1 \\
399.3\end{array}$ & $\begin{array}{l}\text { Bass } \\
\text { Extra-push } \\
\text { Optical }\end{array}$ \\
\hline${ }^{3} \mathrm{He}+{ }^{207} \mathrm{~Pb}$ & ${ }^{210} \mathrm{Po}$ & 67.6 & 10.31 & $\begin{array}{l}23.72 \pm 0.02 \\
23.88 \pm 0.02 \\
24.10 \pm 0.02\end{array}$ & $\begin{array}{l}1.079 \pm 0.001 \\
1.074 \pm 0.001 \\
1.062 \pm 0.001\end{array}$ & $\begin{array}{r}19.2 \\
132.6 \\
183.9\end{array}$ & $\begin{array}{l}\text { Bass } \\
\text { Extra-push } \\
\text { Optical }\end{array}$ \\
\hline${ }^{4} \mathrm{He}+{ }^{206} \mathrm{~Pb}$ & ${ }^{210} \mathrm{Po}$ & 65.3 & 10.31 & $\begin{array}{l}23.91 \pm 0.02 \\
23.77 \pm 0.01 \\
24.04 \pm 0.02\end{array}$ & $\begin{array}{l}1.077 \pm 0.001 \\
1.063 \pm 0.001 \\
1.035 \pm 0.001\end{array}$ & $\begin{array}{l}23.2 \\
54.6 \\
27.3\end{array}$ & $\begin{array}{l}\text { Bass } \\
\text { Extra-push } \\
\text { Optical }\end{array}$ \\
\hline${ }^{3} \mathrm{He}+{ }^{208} \mathrm{~Pb}$ & ${ }^{211} \mathrm{Po}$ & 64.8 & 10.49 & $\begin{array}{l}21.28 \pm 0.02 \\
21.45 \pm 0.02 \\
21.65 \pm 0.02\end{array}$ & $\begin{array}{l}1.081 \pm 0.001 \\
1.077 \pm 0.001 \\
1.064 \pm 0.001\end{array}$ & $\begin{array}{r}16.3 \\
158.9 \\
185.1\end{array}$ & $\begin{array}{l}\text { Bass } \\
\text { Extra-push } \\
\text { Optical }\end{array}$ \\
\hline${ }^{4} \mathrm{He}+{ }^{207} \mathrm{~Pb}$ & ${ }^{211} \mathrm{Po}$ & 63.1 & 10.49 & $\begin{array}{l}21.49 \pm 0.02 \\
21.36 \pm 0.01 \\
21.61 \pm 0.02\end{array}$ & $\begin{array}{l}1.082 \pm 0.001 \\
1.068 \pm 0.001 \\
1.041 \pm 0.001\end{array}$ & $\begin{array}{l}35.4 \\
84.9 \\
41.9\end{array}$ & $\begin{array}{l}\text { Bass } \\
\text { Extra-push } \\
\text { Optical }\end{array}$ \\
\hline${ }^{4} \mathrm{He}+{ }^{208} \mathrm{~Pb}$ & ${ }^{212} \mathrm{Po}$ & 65.5 & 9.61 & $\begin{array}{l}21.97 \pm 0.01 \\
21.78 \pm 0.02 \\
22.11 \pm 0.02\end{array}$ & $\begin{array}{l}1.070 \pm 0.001 \\
1.053 \pm 0.001 \\
1.028 \pm 0.001\end{array}$ & $\begin{array}{l}39.7 \\
48.6 \\
39.2\end{array}$ & $\begin{array}{l}\text { Bass } \\
\text { Extra-push } \\
\text { Optical }\end{array}$ \\
\hline
\end{tabular}


The extracted values of $a_{f} / a_{n}$ lie between 1.0 and 1.1 in all cases, and appear to be the same for different isotopes. The large uncertainties in the formation cross sections seem to be well accommodated by slight variations in the extracted values of $a_{f} / a_{n}$. Other than these observations, it may be premature, at this stage, to attach much more physical significance to the extracted values of $a_{f} / a_{n}$.

For all the systems studied, the $\chi^{2}$ of the fit using the formation cross sections $\left(\sigma_{0}\right)$ from the Bass model [Bass 74$]$ is somewhat smaller than the fits to the same fission excitation function using $\sigma_{0}$ from the Extra-push model [Swia 82] or the Optical model [Macf 78] (see Table 3.1). This alone, of course, is not sufficient to indicate that the Bass model prediction for the fusion cross sections is better than the other models. However, there are indications that even the Bass model may overestimate the fusion cross sections [Stic 74], while the fusion cross sections predicted by the Bass model are already smaller than those predicted by the Extrapush model and the Optical model.

The fission excitation functions of the nuclei ${ }^{209,210,211}$ Po produced in the ${ }^{3} \mathrm{He}+{ }^{206,207,208} \mathrm{~Pb}$ reactions, and the corresponding fits with the three different assumptions for the formation cross sections of the fissioning nuclei, are shown in Fig. 3.5. The fission barriers $B_{f}$ and the ratio $a_{f} / a_{n}$ extracted from the fits are listed in Table 3.1. The formation cross sections of ${ }^{210} \mathrm{Po}$ from the reaction ${ }^{3} \mathrm{He}+{ }^{207} \mathrm{~Pb}$ which are used in the fits and predicted by the Bass, the Extra-push and the Optical models, are shown in Fig. 3.2. As in the case of osmium isotopes, the formation cross sections of ${ }^{209,211} \mathrm{Po}$ from the reactions ${ }^{3} \mathrm{He}+{ }^{206,208} \mathrm{~Pb}$ are taken to be the same 
as those of ${ }^{210} \mathrm{Po}$ from the reaction ${ }^{3} \mathrm{He}+{ }^{207} \mathrm{~Pb}$ at the same incident energy. In the data range where the fitting was attempted, the fits using the formation cross sections from the Bass model are excellent for all three polonium nuclei $\left({ }^{209,210,211} \mathrm{Po}\right)$. The quality of the fits using the formation cross sections $\left(\sigma_{0}\right)$ from the Extra-push model and the Optical model are, however, not as good as the quality found in the fit with $\sigma_{0}$ from the Bass model. For all the three nuclei, the $\chi^{2}$ of the fits using the formation cross sections from the Bass model [Bass 74] is substantially smaller than that of the fits using $\sigma_{0}$ from the Extra-push [Swia 82] or the Optical models [Macf 78] (see Table 3.1). Nevertheless, the fission barriers $\left(B_{f}^{*}\right)$ extracted using the different formation cross sections are remarkably consistent, despite the large uncertainties in the formations cross sections, as seen in the cases of osmium isotopes. For different polonium isotopes, the extracted $a_{f} / a_{n}$ appears to be the same.

The fission excitations of the nuclei ${ }^{210,211,212} \mathrm{Po}$ produced in the ${ }^{4} \mathrm{He}+$ ${ }^{206,207,208} \mathrm{~Pb}$ reactions, and the corresponding fits with the same three assumptions for the formation cross sections of the fissioning nuclei, are shown in Fig. 3.6. The fission barriers $B_{f}^{*}$ and the ratio $a_{f} / a_{n}$ extracted from the fits are also listed in Table 3.1 . The formation cross sections of ${ }^{211} \mathrm{Po}$ from the reaction ${ }^{4} \mathrm{He}+{ }^{207} \mathrm{~Pb}$ which are used in the fits and predicted by the Bass, the Extra-push and the Optical models, are shown in Fig. 3.3. The formation cross sections of ${ }^{210,212} \mathrm{Po}$ from the reactions ${ }^{4} \mathrm{He}$ $+{ }^{206,208} \mathrm{~Pb}$ are taken to be the same as those of ${ }^{211} \mathrm{Po}$ from the reaction ${ }^{4} \mathrm{He}+{ }^{207} \mathrm{~Pb}$ at the same incident energy. In the data range where the fitting was attempted, all the fits are excellent, despite the large differences in the formation cross sections 
predicted by the different models. For all three nuclei $\left({ }^{210,211,212} \mathrm{Po}\right)$, the $\chi^{2}$ values of the fits using the formation cross sections $\left(\sigma_{0}\right)$ from the different models are about the sarne (see Table 3.1).

As in the cases of Os and Po nuclei produced in ${ }^{3} \mathrm{He}$-induced reactions, the fission barriers $\left(B_{f}^{*}\right)$ for Po nuclei produced in ${ }^{4} \mathrm{He}$-induced reactions extracted using the different formation cross sections are consistent to within $0.3 \mathrm{MeV}$, despite the large differences in the formations cross sections. More remarkably (and to one's own satisfaction), the extracted fission barriers for the same nuclei ( ${ }^{210} \mathrm{Po}$ or ${ }^{211} \mathrm{Po}$ ) produced in different entrance channels $\left({ }^{3} \mathrm{He} /{ }^{4} \mathrm{He}\right.$-induced) are consistent within 0.2 $\mathrm{MeV}$. Thus, we have found a simple and robust method to determine accurately the fission barriers from the fission excitation functions, given the shell effects of the nuclei concerned.

\subsection{Shell Effects}

The shell effect of a nucleus is, conventionally, defined as the difference between its ground state mass and the corresponding macroscopic liquid-drop value. The standard procedure to determine the shell effect of a nucleus is, therefore, to develop a liquid-drop model of nuclei, and to take the difference between the ground state mass of the nucleus and the liquid-drop baseline. It is difficult, however, to establish a good liquid-drop baseline. Great efforts have been made to develop and improve the various liquid-drop models. As the models evolved, so did the derived 
shell effects. Over the years, the values for the shell effects have changed quite substantially [Myer 65, Möll 93, Myer 94]. The shell effects given by the Finite Range Droplet Model (FRDM) by Möller et al [Möll 93, Myer 94] represent the culmination of over 30 years of effort.

The standard procedure to determine shell effects is necessarily a global one, i.e., the shell effect of a nucleus depends on the properties of all the other nuclei. The parameters in a liquid-drop model are determined by fitting the liquid-drop model to the ground state masses of all the known nuclei across the nuclidic chart. The parameters are adjusted to achieve a best fit on a global basis, but the same fit may not be the best fit for a local mass region. This may account, in part, for changes in the derived shell effects over the years:

The shell effects affect the available phase space of an excited nucleus [Rose 57, Huiz 72] (see discussion in Section 1.2). In particular, the shell effect $\Delta_{\text {shell }}^{n-1}$ of the daughter nucleus produced by neutron evaporation goes, asymptotically, into the argument of the level density expression when the excitation energy is a few $\mathrm{MeV}$ above the shell effects (see Eq. 1.14). Consequently, the shell effect $\Delta_{\text {shell }}^{n-1}$ exerts great influence on the competition between fission and neutron emission, and together with the fission barrier $B_{f}$ determines the branching ratio of fission. Using $\Delta_{\text {shell }}^{n-1}$ as a free parameter, the fit of the statistical formalism for the fission cross sections to the experimental fission excitation function should allow us to extract, independently and locally, the shell effect of the daughter nucleus produced by evaporation of a neutron from the fissioning nucleus. 
In Section 3.2, it was shown that, using $\Delta_{\text {shell }}^{n-1}$ taken from ref. [Möll 93] as a fixed parameter, the fits to the low-energy portion of the fission excitation function produced remarkably consistent fission barriers $\left(B_{f}^{*}\right)$ of a fissioning nucleus, regardless of the substantial uncertainties in the formation cross sections of the nucleus.

In this section, it will be demonstrated that, given the effective fission barrier $B_{f}^{*}$, one can extract, to a very small uncertainty, the shell effect of the residual nucleus produced by neutron evaporation, by fitting the fission excitation function with the statistical formalism, using $\Delta_{\text {shell }}^{n-1}$ and $a_{f} / a_{n}$ as free parameters. For this purpose, the fits should also be limited to only the low-energy portion of the excitation function, in order to avoid complications from the possible involvement of multichance fission.

The fission barrier $B_{f}^{*}$ to be used as a fixed parameter in the fitting can be either the nominal values calculated with the liquid drop models [Möll 93, Myer 94], or the experimental values. The experimental determination of the fission barriers is possible by measuring the fission excitation functions to very low energies. When the fission excitation functions are measured very close to the fission barriers, the true fission barriers may be determined, regardless of the uncertainties in the formation cross sections of the fissioning nuclei, and regardless of the fact that the relevant shell effects are yet to be determined. To measure the fission excitation functions very close to the fission barriers is difficult, due to the extremely small cross sections $\left(<10^{-7} \mathrm{mb}\right)$, and requires a large amount of beam time. For quite a few nuclei produced in ${ }^{4} \mathrm{He}$-induced reactions on the targets of ${ }^{197} \mathrm{Au},{ }^{206,207,208} \mathrm{~Pb}$, and ${ }^{209} \mathrm{Bi}$, great efforts have been undertaken to measure their fission excitation functions from 
hundreds of $\mathrm{mb}$ (above $100 \mathrm{MeV}$ in excitation energy) all the way down to fission cross sections of $10^{-8} \mathrm{mb}$, and from the measured fission excitation functions, their true fission barriers $B_{f}$ have been determined [Khod 66, More 72a, More 74].

For the purpose of demonstrating the method by which the shell effect can be determined from the fission excitation function, the effective barriers $B_{f}^{*}$ presented in the previous section are used instead as a fixed parameter. The values of $B_{f}^{*}$ chosen for the fits are those extracted using the formation cross sections predicted by the Bass model. If the fissioning nuclei $\left({ }^{210} \mathrm{Po}\right.$ or $\left.{ }^{211} \mathrm{Po}\right)$ are produced by the different entrance channels ( ${ }^{3} \mathrm{He}$ - and ${ }^{4} \mathrm{He}$-induced), the barriers determined from the ${ }^{4} \mathrm{He}-$ induced fission excitation functions are then used. The $B_{f}^{*}$ values used in the fitting are given in Table 3.2 .

Now a fit can be carried out readily with two free parameters: $\Delta_{\text {shell }}^{n-1}-$ the shell effect of the residual nucleus produced by neutron emission, and $a_{f} / a_{n}-$ the ratio of the level density parameter at the saddle point to that of the compound nucleus at its ground state configurations. As in Section 3.2, a level density parameter $a_{n}=A / 8\left(\mathrm{MeV}^{-1}\right)$ was assumed for the compound nuclei. The fits were also limited to the low-energy segment of the measured fission excitation function, to ensure the dominance of the first-chance fission. The energy range of the data points used in the fitting was chosen to be the same as that used in the fits in Section 3.2. The fission excitation functions and the corresponding fits for osmium isotopes produced in ${ }^{3} \mathrm{He}$-induced reactions, and for polonium isotopes produced in ${ }^{3} \mathrm{He} /{ }^{4} \mathrm{He}$-induced reactions, are shown in Figs. 3.7, 3.8 and 3.9, respectively. 
These fits with $\Delta_{\text {shell }}^{n-1}$ and $a_{f} / a_{n}$ as free parameters look similar to the fits using $B_{f}^{*}$ and $a_{f} / a_{n}$ as free parameters. For nuclei ${ }^{185,186,187,189}$ Os produced in ${ }^{3} \mathrm{He}$ $+{ }^{182,183,184,186} \mathrm{~W}$ reactions, in the data range where the fits were attempted, the fits shown in Fig. 3.7 and the fits in Fig. 3.4 look almost identical. Only in the extensions of the fits to higher energies, can one discern some small differences. The same can be said for the nuclei ${ }^{209,210,211} \mathrm{Po}$ produced in ${ }^{3} \mathrm{He}+{ }^{206,207,208} \mathrm{~Pb}$ reactions (see Fig. 3.8 and Fig. 3.5), and for the nuclei ${ }^{210,211,212} \mathrm{Fo}$ produced in ${ }^{4} \mathrm{He}+{ }^{206,207,208} \mathrm{~Pb}$ reactions (see Fig. 3.9 and Fig. 3.6).

The shell effects $\Delta_{\text {shell }}^{n-1}$ and the $a_{f} / a_{n}$ values, extracted from the fits shown in Figs. 3.7, 3.8 and 3.9, are listed in Table 3.2. The shell effects $\Delta_{\text {shell }}^{n-1}$, extracted using the different formation cross sections, show excellent consistency, despite the large differences in the formation cross sections used in the fitting (see Figs. 3.1, 3.2 and 3.3i). The consistency is retained remarkably well for the same nuclei produced in different entrance channel reactions. For example, the shell effect for the nucleus ${ }^{209} \mathrm{Po}$, extracted from the fission excitation function of the fissioning nucleus ${ }^{210} \mathrm{Po}$ produced in ${ }^{3} \mathrm{He}+{ }^{207} \mathrm{~Pb}$ reaction, are $10.56,10.38$, and $10.06 \mathrm{MeV}$ with use of the formation cross sections from the Bass [Bass 74], the Extra-push [Swia 82], and the Optical models [Macf 78], respectively. The shell effect for the same nucleus $\left({ }^{209} \mathrm{Po}\right)$, extracted from the fission excitation function of the fissioning nucleus ${ }^{210} \mathrm{Po}$ produced in ${ }^{4} \mathrm{He}+{ }^{206} \mathrm{~Pb}$ reaction, are $10.31,10.53$, and $10.12 \mathrm{MeV}$ by using the different formation cross sections, respectively. These values are consistent, to within 0.5 $\mathrm{MeV}$, which must be considered very good indeed, since they were extracted from the 


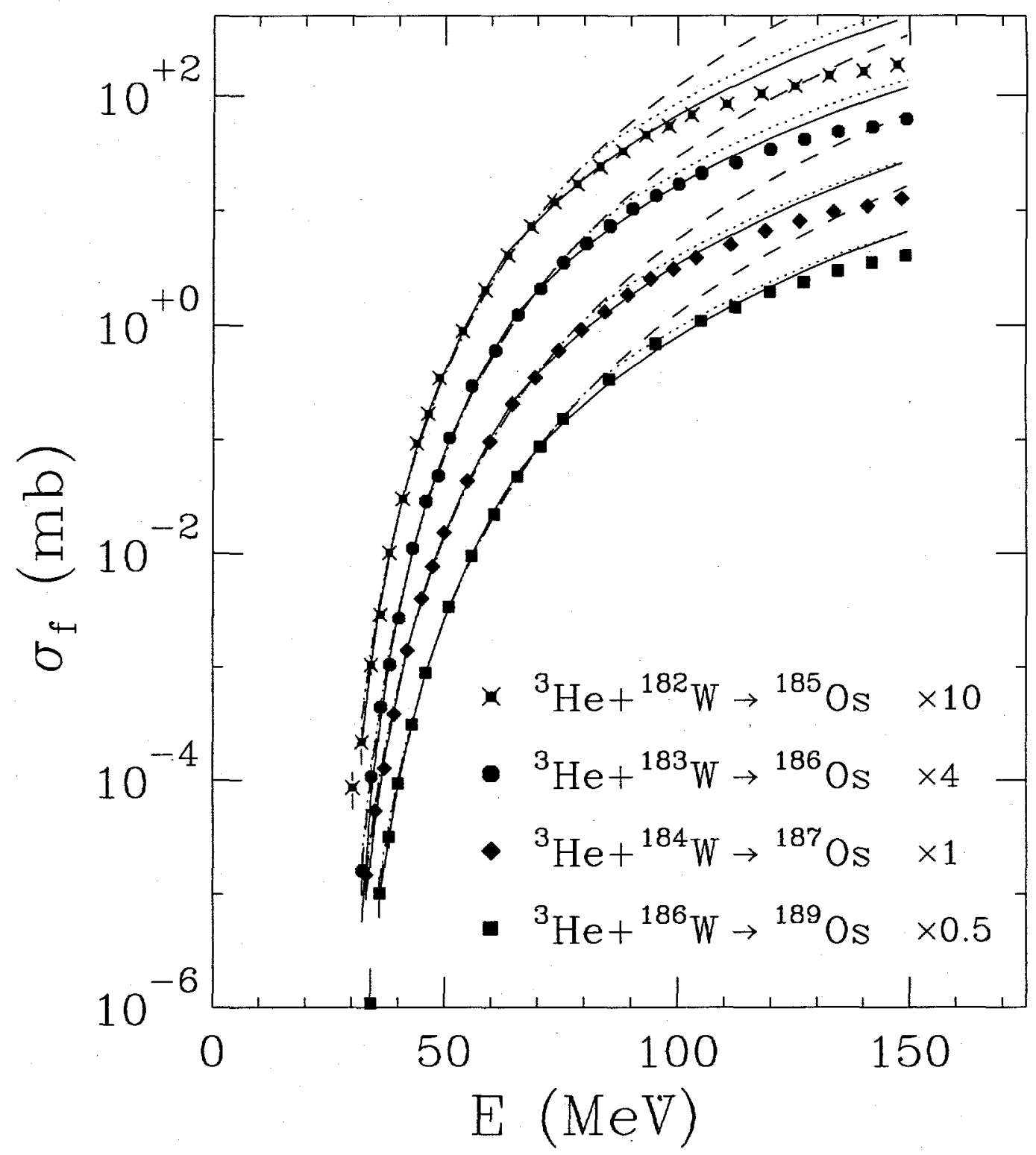

Figure 3.7: The fission excitation functions for the nuclei ${ }^{185,186,187,189} \mathrm{Os}$ produced in the reactions ${ }^{3} \mathrm{He}+{ }^{182,183,184,186} \mathrm{~W}$, were fitted with the statistical formalism, with two free parameters: $\Delta_{\text {shell }}^{n-1}$ and $a_{f} / a_{n}$. The fission Barrier $B_{f}^{*}$ listed in Table 3.2 was used as a fixed parameter. The solid lines, the dotted lines and the dashed lines represent fits using the formation cross sections of the fissioning nuclei calculated by the Bass, the Extra-push and the Optical models, respectively. The fits were attempted for only the low energy portion of the fission excitation functions. The highest energy of the data segment used in the fitting is indicated by $E_{H}$ in Table 3.2. The fits were extended to the full range of the experimental data, using the extracted parameters listed in Table 3.2. The number to the right of each legend indicates the factor by which the corresponding excitation function is multiplied for visual clarity. 


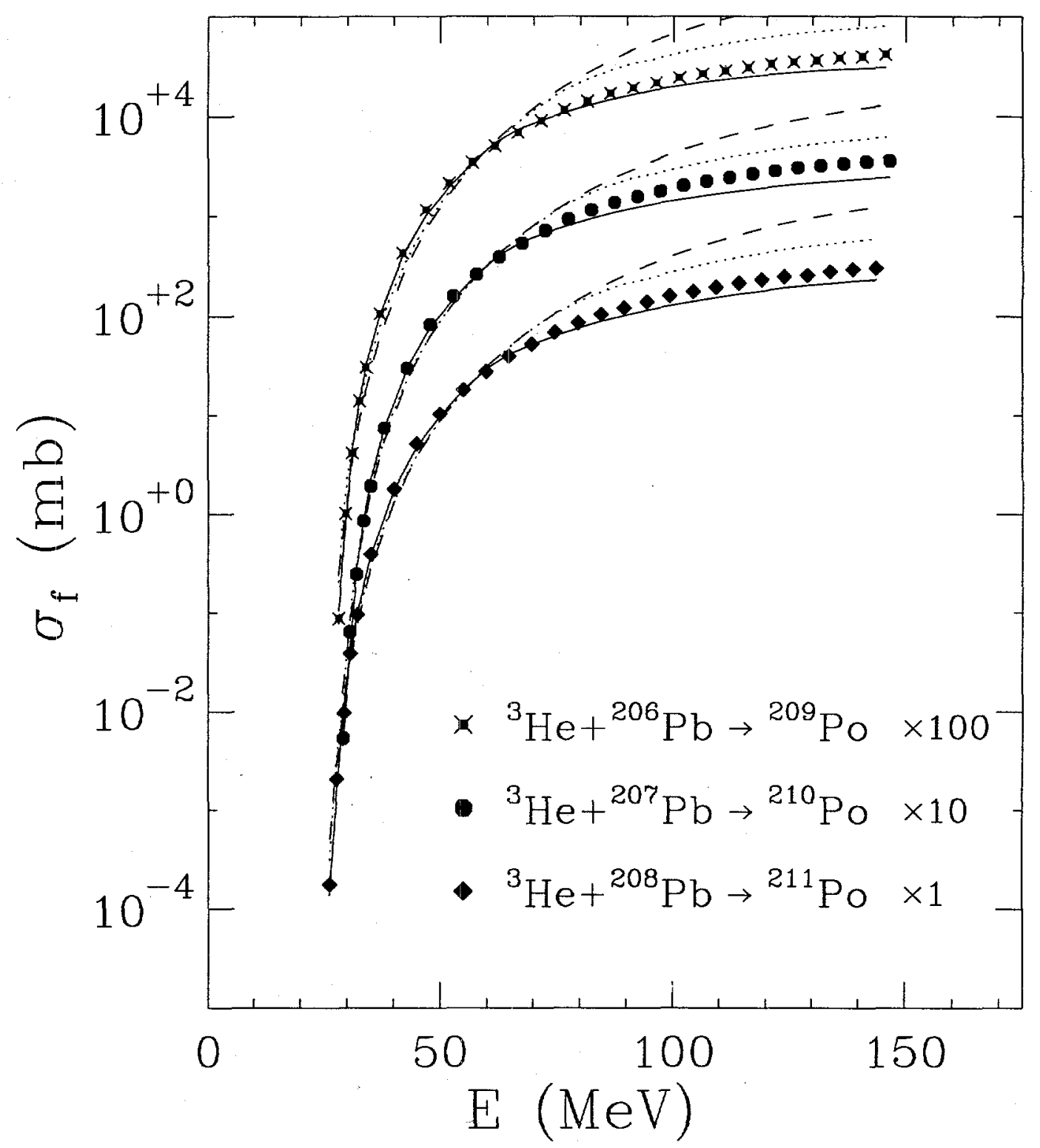

Figure 3.8: The fission excitation functions for the nuclei ${ }^{209,210,211} \mathrm{Po}$ produced in the reactions ${ }^{3} \mathrm{He}+{ }^{206,207,208} \mathrm{~Pb}$, were fitted with the statistical formalism, with two free parameters: $\Delta_{\text {shell }}^{n-1}$ and $a_{f} / a_{n}$. The fission Barrier $B_{f}^{*}$ listed in Table 3.2 was used as a fixed parameter. The solid lines, the dotted lines and the dashed lines represent fits using the formation cross sections of the fissioning nuclei calculated by the Bass, the Extra-push and the Optical models, respectively. The fits were attempted for only the low energy portion of the fission excitation functions. The highest energy of the data segment used in the fitting is indicated by $E_{H}$ in Table 3.2. The fits were extended to the full range of the experimental data, using the extracted parameters listed in Table 3.2. The number to the right of each legend indicates the factor by which the corresponding excitation function is multiplied for visual clarity. 


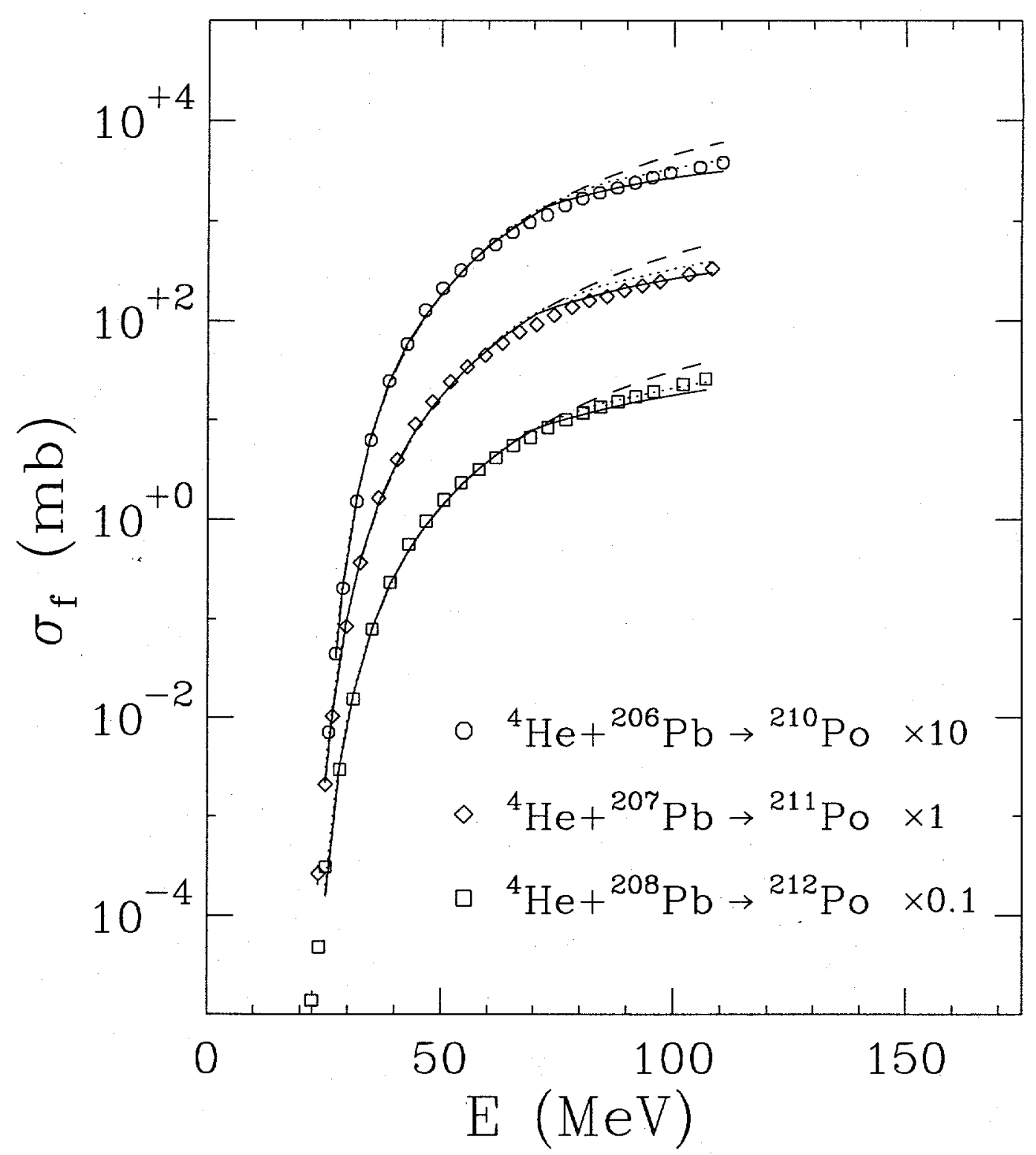

Figure 3.9: The fission excitation functions for the nuclei ${ }^{210,211,212} \mathrm{Po}$ produced in the reactions ${ }^{4} \mathrm{He}+{ }^{206,207,208} \mathrm{~Pb}$, were fitted with the statistical formalism, with two free parameters: $\Delta_{\text {shell }}^{n-1}$ and $a_{f} / a_{n}$. The fission Barrier $B_{f}^{*}$ listed in Table 3.2 was used as a fixed parameter. The solid lines, the dotted lines and the dashed lines represent fits using the formation cross sections of the fissioning nuclei calculated by the Bass, the Extra-push and the Optical models, respectively. The fits were attempted for only the low energy portion of the fission excitation functions. The highest energy of the data segment used in the fitting is indicated by $E_{H}$ in Table 3.2. The fits were extended to the full range of the experimental data, using the extracted parameters listed in Table 3.2. The number to the right of each legend indicates the factor by which the corresponding excitation function is multiplied for visual clarity. 
Table 3.2: The shell effects $\Delta_{\text {shell }}^{n-1}$ of daughter nuclei produced by neutron emission from the fissioning nuclei, and the values of $a_{f} / a_{n}$, were extracted by fitting the low energy portion of the measured excitation functions. For comparison, the nominal shell effects $\Delta_{\text {FRDM }}^{n-1}$ given in ref. [Möll 93] are also listed. The data and the corresponding fits are shown in Figs. 3.7, 3.8 and 3.9. The fits were performed with three different assumptions for the formation cross sections of the fissioning compound nuclei (CN): the Bass, the Extra-push and the Optical models. $a_{n}=A / 8\left(\mathrm{MeV}^{-1}\right)$ was assumed. $B_{f}^{*}(\mathrm{MeV})$ are the effective fission barriers taken from Table 3.1 and used as a fixed parameter in the fitting. $E_{H}$ $(\mathrm{MeV})$ indicates the highest excitation energy of the data points up to which the fits were attempted.

\begin{tabular}{|c|c|c|c|c|c|c|c|c|}
\hline Reaction & $\mathrm{CN}$ & $E_{H}$ & $B_{f}^{*}$ & $\Delta_{\text {FRDM }}^{n-1}$ & $\Delta_{\text {shell }}^{n-1}(\mathrm{MeV})$ & $a_{f} / a_{n}$ & $\chi^{2}$ & $\sigma_{0}$ \\
\hline${ }^{3} \mathrm{He}+{ }^{182} \mathrm{~W}$ & ${ }^{185} \mathrm{Os}$ & 73.4 & 21.43 & 0.85 & $\begin{array}{l}0.86 \pm 0.02 \\
1.46 \pm 0.02 \\
1.28 \pm 0.07\end{array}$ & $\begin{array}{l}1.103 \pm 0.001 \\
1.075 \pm 0.001 \\
1.060 \pm 0.002\end{array}$ & $\begin{array}{r}8.4 \\
34.0 \\
27.1\end{array}$ & $\begin{array}{l}\text { Bass } \\
\text { Extra-push } \\
\text { Optical }\end{array}$ \\
\hline${ }^{3} \mathrm{He}+{ }^{183} \mathrm{~W}$ & ${ }^{186} \mathrm{Os}$ & 75.5 & 23.42 & 1.18 & $\begin{array}{l}1.18 \pm 0.02 \\
1.79 \pm 0.07 \\
1.61 \pm 0.06\end{array}$ & $\begin{array}{l}1.095 \pm 0.001 \\
1.066 \pm 0.002 \\
1.052 \pm 0.002\end{array}$ & $\begin{array}{l}13.5 \\
28.6 \\
22.5\end{array}$ & $\begin{array}{l}\text { Bass } \\
\text { Extra-push } \\
\text { Optical }\end{array}$ \\
\hline${ }^{3} \mathrm{He}+{ }^{184} \mathrm{~W}$ & ${ }^{187} \mathrm{Os}$ & 74.4 & 22.33 & 1.32 & $\begin{array}{l}1.32 \pm 0.02 \\
2.07 \pm 0.07 \\
1.86 \pm 0.02\end{array}$ & $\begin{array}{l}1.098 \pm 0.001 \\
1.066 \pm 0.002 \\
1.053 \pm 0.001\end{array}$ & $\begin{array}{r}6.0 \\
19.0 \\
15.1\end{array}$ & $\begin{array}{l}\text { Bass } \\
\text { Extra-push } \\
\text { Optical }\end{array}$ \\
\hline${ }^{3} \mathrm{He}+{ }^{186} \mathrm{~W}$ & ${ }^{189} \mathrm{Os}$ & 75.4 & 23.23 & 1.87 & $\begin{array}{l}1.86 \pm 0.03 \\
2.66 \pm 0.02 \\
2.44 \pm 0.02\end{array}$ & $\begin{array}{l}1.091 \pm 0.001 \\
1.058 \pm 0.001 \\
1.045 \pm 0.001\end{array}$ & $\begin{array}{l}4.6 \\
4.6 \\
3.1\end{array}$ & $\begin{array}{l}\text { Bass } \\
\text { Extra-push } \\
\text { Optical }\end{array}$ \\
\hline${ }^{3} \mathrm{He}+{ }^{206} \mathrm{~Pb}$ & ${ }^{209} \mathrm{Po}$ & 66.6 & 21.80 & 9.06 & $\begin{array}{l}9.06 \pm 0.03 \\
8.80 \pm 0.03 \\
8.23 \pm 0.03\end{array}$ & $\begin{array}{l}1.086 \pm 0.001 \\
1.083 \pm 0.001 \\
1.080 \pm 0.001\end{array}$ & $\begin{array}{r}24.3 \\
222.9 \\
481.4\end{array}$ & $\begin{array}{l}\text { Bass } \\
\text { Extra-push } \\
\text { Optical }\end{array}$ \\
\hline${ }^{3} \mathrm{He}+{ }^{207} \mathrm{~Pb}$ & ${ }^{210} \mathrm{Po}$ & 67.6 & 23.91 & 10.31 & $\begin{array}{l}10.56 \pm 0.03 \\
10.38 \pm 0.03 \\
10.06 \pm 0.03\end{array}$ & $\begin{array}{l}1.077 \pm 0.001 \\
1.073 \pm 0.001 \\
1.063 \pm 0.001\end{array}$ & $\begin{array}{r}18.9 \\
130.2 \\
203.8\end{array}$ & $\begin{array}{l}\text { Bass } \\
\text { Extra-push } \\
\text { Optical }\end{array}$ \\
\hline${ }^{4} \mathrm{He}+{ }^{206} \mathrm{~Pb}$ & ${ }^{210} \mathrm{Po}$ & 65.3 & 23.91 & 10.31 & $\begin{array}{l}10.31 \pm 0.02 \\
10.53 \pm 0.02 \\
10.12 \pm 0.02\end{array}$ & $\begin{array}{l}1.077 \pm 0.001 \\
1.061 \pm 0.001 \\
1.036 \pm 0.001\end{array}$ & $\begin{array}{l}23.2 \\
46.4 \\
29.5\end{array}$ & $\begin{array}{l}\text { Bass } \\
\text { Extra-push } \\
\text { Optical }\end{array}$ \\
\hline${ }^{3} \mathrm{He}+{ }^{208} \mathrm{~Pb}$ & ${ }^{211} \mathrm{Po}$ & 64.8 & 21.49 & 10.49 & $\begin{array}{l}10.79 \pm 0.03 \\
10.57 \pm 0.03 \\
10.28 \pm 0.03\end{array}$ & $\begin{array}{l}1.080 \pm 0.001 \\
1.076 \pm 0.001 \\
1.065 \pm 0.001\end{array}$ & $\begin{array}{r}14.4 \\
155.4 \\
202.1\end{array}$ & $\begin{array}{l}\text { Bass } \\
\text { Extra-push } \\
\text { Optical }\end{array}$ \\
\hline${ }^{4} \mathrm{He}+{ }^{207} \mathrm{~Pb}$ & ${ }^{211} \mathrm{Po}$ & 63.1 & 21.49 & 10.49 & $\begin{array}{l}10.49 \pm 0.02 \\
10.71 \pm 0.02 \\
10.32 \pm 0.02\end{array}$ & $\begin{array}{l}1.082 \pm 0.001 \\
1.066 \pm 0.001 \\
1.042 \pm 0.001\end{array}$ & $\begin{array}{l}35.4 \\
73.0 \\
48.0\end{array}$ & $\begin{array}{l}\text { Bass } \\
\text { Extra-push } \\
\text { Optical }\end{array}$ \\
\hline${ }^{4} \mathrm{He}+{ }^{208} \mathrm{~Pb}$ & ${ }^{212} \mathrm{Po}$ & 65.5 & 21.97 & 9.61 & $\begin{array}{l}9.61 \pm 0.03 \\
9.87 \pm 0.03 \\
9.42 \pm 0.03\end{array}$ & $\begin{array}{l}1.070 \pm 0.001 \\
1.051 \pm 0.001 \\
1.029 \pm 0.001\end{array}$ & $\begin{array}{l}39.7 \\
46.2 \\
38.4\end{array}$ & $\begin{array}{l}\text { Bass } \\
\text { Extra-push } \\
\text { Optical }\end{array}$ \\
\hline
\end{tabular}


fission excitation functions for different reaction channels, and were extracted using substantially different formation cross section estimates. The same consistency, to within $0.8 \mathrm{MeV}$ in general, is observed for all the other nuclei studied in this thesis (see Table 3.2).

Thus, a new method has been established to determine, with very small uncertainty, the shell effect of the daughter nucleus produced by neutron emission from a fissioning nucleus, given the fission barrier. This new method, based on detailed analyses of the fission excitation functions, is totally independent of the standard procedure, and is completely local. It depends only on the properties of the nucleus under consideration.

As observed in the previous section, the extracted values of $a_{f} / a_{n}$ lie between 1.0 and 1.1, and $a_{f} / a_{n}$ appears to be the same for different isotopes. The large differences in the formation cross sections seem to be well accommodated by slight variations in the extracted values of $a_{f} / a_{n}$.

\subsection{Simultaneous Determination of the Fission Barrier and}

\section{the Shell Effect}

In the previous sections, it has been shown that the fission barrier $B_{f}^{*}$ can be very well determined given the shell effect $\Delta_{\text {shell }}^{n-1}$, and that the shell effect $\Delta_{\text {shell }}^{n-1}$ can be equally well determined given the fission barrier $B_{f}^{*}$, by fitting the experimental fission excitation function, regardless of the current inadequacy in the knowledge of 
the formation cross sections of the fissioning nucleus.

Naturally, one would like to determine the fission barrier $B_{f}^{*}$ and the shell effect $\Delta_{\text {shell }}^{n-1}$ simultaneously, by using both of them and $a_{f} / a_{n}$ as free parameters in the fit. Previously, we have fitted the fission excitation functions measured in $1970 \mathrm{~s}$ for fourteen nuclei produced in ${ }^{4} \mathrm{He}$-induced reactions [More 95b], with these three free parameters. The shell effects extracted from the fits correlate remarkably well with those determined from the ground state masses. The extracted effective fission barriers $B_{f}^{*}$ are consistent with the relationship $B_{f}^{*}=B_{f}+\frac{1}{2} g \Delta_{0}^{2}$ for even-even nuclei and $B_{f}^{*}=B_{f}+\frac{1}{2} g \Delta_{0}^{2}-\Delta_{0}$ for odd $A$ nuclei for a value of $\Delta_{0} \sim 0.7 \mathrm{MeV}$.

These fits were performed not just for the low-energy portion of the measured fission excitation functions, but for the excitation functions from a few $\mathrm{MeV}$ above the fission barriers to the highest energy data point measured $(\sim 120 \mathrm{MeV})$. Although multichance fission is expected to occur at high energies, these fits, which included only first chance fission, were excellent across the whole range of energy where the fits were attempted. The scaling law, predicted by the transition state rate for first chance fission, was found to be well observed by the total fission probabilities. This puzzling aspect has created much concern and anticipation regarding how first chance fission probabilities should behave. The argument was thus made that first chance fission may be substantially suppressed by a transient time and that the effects of the transient time were somehow compensated by fission probabilities of higher chances [Back 97].

Here, as was done earlier in the two free parameter fits, the three free pa- 
rameter fit was performed for only the low-energy portion of the fission excitation functions, to ensure the dominance of first chance fission, thus avoiding the difficulties associated with multichance fission. The three parameter fit, in principle, should allow us to determine $B_{f}^{*}, \Delta_{\text {shell }}^{n-1}$, and $a_{f} / a_{n}$, simultaneously, and perhaps, with good consistency as well.

Shown in Fig. 3.10 are the fission excitation functions and the corresponding fits for polonium isotopes ${ }^{210,211,212} \mathrm{Po}$ produced in ${ }^{4} \mathrm{He}+{ }^{206,207,208} \mathrm{~Pb}$ reactions. The fits were performed with the same three different assumptions for the formation cross sections of the fissoning nuclei: the Bass, the Extra-push and the Optical models. The formation cross sections given by these models were shown previously in Fig. 3.3. The energy range of the excitation functions in which the three parameter fits were attempted was chosen to be the same as in the two parameter fits (see Figs. 3.6 \& 3.9 , and Tables $3.1 \& 3.2$ ).

All fits shown in Fig. 3.10 are excellent in the energy range where the data were used in the fitting. Notice that the extensions to higher energies of the fits using the formation cross sections from the Bass and the Extra-push models agree well with the data points. The extensions of the fits using the formation cross sections from the Optical model are all higher than the experimental data, as was the case in the two parameter fits.

The fission barrier $B_{f}^{*}$, the shell effect of the daughter nucleus after neutron emission $\Delta_{\text {shell }}^{n-1}$, and $a_{f} / a_{n}$, determined from these fits for the fissioning nuclei ${ }^{210,211,212} \mathrm{Po}$, are listed in Table 3.3. Both the $B_{f}^{*}$ and the $\Delta_{\text {shell }}^{n-1}$, extracted using 


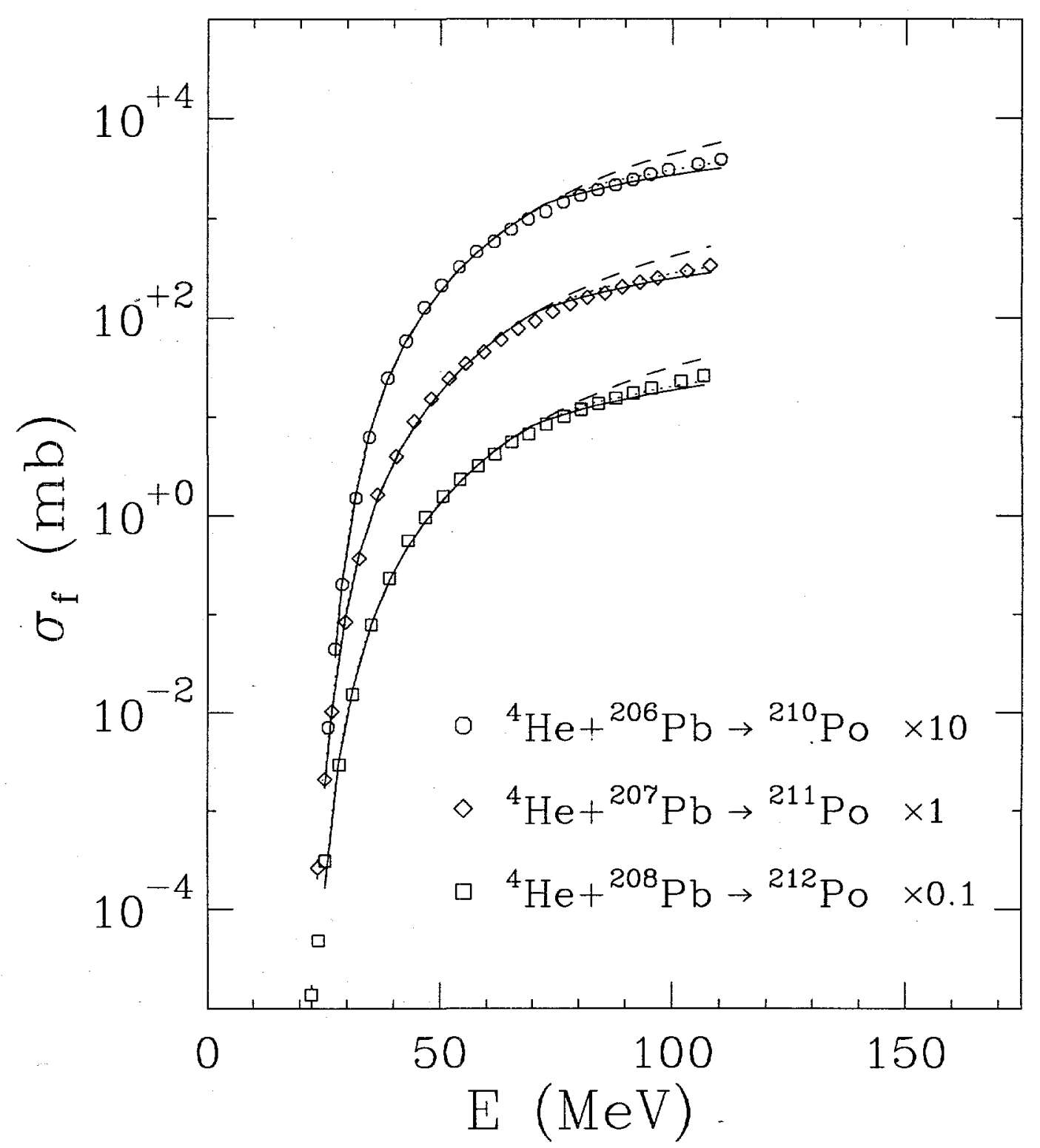

Figure 3.10: The fission excitation functions for the nuclei ${ }^{210,211,212}$ Po produced in the reactions ${ }^{4} \mathrm{He}+{ }^{206,207,208} \mathrm{~Pb}$, were fitted with the statistical formalism, with $B_{f}^{*}, \Delta_{\text {shell }}^{n-1}$, and $a_{f} / a_{n}$ as the three free parameters. The solid lines, the dotted lines and the dashed lines represent fits using the formation cross sections of the fissioning nuclei calculated by the Bass, the Extra-push and the Optical models, respectively. The fits were attempted for only the low energy portion of the fission excitation functions. The highest energy of the data segment used in the fitting is indicated by $E_{H}$ in Table 3.3. The fits were extended to the full range of the experimental data, using the extracted parameters $\left(B_{f}^{*}, \Delta_{\text {shell }}^{n-1}\right.$, and $a_{f} / a_{n}$ ) which are listed in Table 3.3. The number to the right of each legend indicates the factor by which the corresponding excitation function is multiplied for visual clarity. 
Table 3.3: The effective fission barriers $B_{f}^{*}$, the shell effects $\Delta_{\text {shell }}^{n-1}$ of the residual nuclei after neutron emission, and the ratios $a_{f} / a_{n}$, extracted for polonium isotopes produced in the ${ }^{4} \mathrm{He}$-induced reactions by fitting the low energy portion of the measured fission excitation functions. The data and the corresponding fits are shown in Fig. 3.10. The fits were performed with three different assumptions for the formation cross sections $\sigma_{0}$ of the fissioning compound nuclei $(\mathrm{CN})$ : the Bass, the Extra-push and the Optical models. $a_{n}=A / 8\left(\mathrm{MeV}^{-1}\right)$ was assumed. $E_{H}(\mathrm{MeV})$ indicates the highest excitation energy of the data points up to which the fits were attempted.

\begin{tabular}{lcc|ccccc|l}
\hline \hline \multirow{2}{*}{ Reaction } & \multirow{2}{*}{${ }^{2}$} & $E_{H}$ & $B_{f}^{*}$ & $\Delta_{\text {shell }}^{n-1}$ & $B_{f}^{*}-\Delta_{\text {shell }}^{n-1}$ & $a_{f} / a_{n}$ & $\chi^{2}$ & \multicolumn{1}{|c}{$\sigma_{0}$} \\
\hline \multirow{3}{*}{${ }^{4} \mathrm{He}+{ }^{206} \mathrm{~Pb}$} & ${ }^{210} \mathrm{Po}$ & 65.3 & 23.99 & 10.42 & 13.57 & 1.076 & 23.1 & Bass \\
& & & 24.43 & 11.28 & 13.15 & 1.054 & 32.6 & Extra-push \\
& & & 24.16 & 10.49 & 13.67 & 1.033 & 26.8 & Optical \\
\hline \multirow{3}{*}{${ }^{4} \mathrm{He}+{ }^{207} \mathrm{~Pb}$} & \multirow{2}{*}{${ }^{211} \mathrm{Po}$} & 63.1 & 21.87 & 11.06 & 10.81 & 1.077 & 28.8 & Bass \\
& & & 22.28 & 11.89 & 10.39 & 1.055 & 39.3 & Extra-push \\
& & 22.02 & 11.09 & 10.93 & 1.035 & 33.1 & Optical \\
\hline \multirow{3}{*}{${ }^{4} \mathrm{He}+{ }^{208} \mathrm{~Pb}$} & \multirow{2}{*}{${ }^{212} \mathrm{Po}$} & \multirow{2}{*}{65.5} & 21.77 & 9.33 & 12.44 & 1.071 & 39.0 & Bass \\
& & & 22.24 & 10.24 & 12.00 & 1.049 & 45.0 & Extra-push \\
\hline \hline
\end{tabular}

different formation cross sections, show remarkable consistency, regardless of the substantial difference in the formation cross sections. The extracted barriers $B_{f}^{*}$ are consistent within $0.5 \mathrm{MeV}$, and the extracted shell effects are consistent within 1.0 $\mathrm{MeV}$. For instance, for the nucleus ${ }^{211} \mathrm{Po}$ produced in the reaction ${ }^{4} \mathrm{He}+{ }^{207} \mathrm{~Pb}$, the values for $B_{f}^{*}$ are $21.87,22.28$, and $22.02 \mathrm{MeV}$, and the values for $\Delta_{\text {shell }}^{n-1}$ are 11.06, 11.89, 11.09 MeV, when extracted from the fits using formation cross sections predicted by the Bass, Extra-push, and Optical models, respectively. The large uncertainties in the formation cross sections are found to be well accommodated with slight variations of $a_{f} / a_{n}$.

For nuclei ${ }^{209,210,211} \mathrm{Po}$ produced in the ${ }^{3} \mathrm{He}$-induced reactions on ${ }^{206,207,208} \mathrm{~Pb}$, 
however, the fission barriers $B_{f}^{*}$ and the shell effects $\Delta_{\text {shell }}^{n-1}$ extracted from the three parameter fits are less consistent. The $B_{f}^{*}, \Delta_{\text {shell }}^{n-1}$ and $a_{f} / a_{n}$ extracted from the fits shown in Fig. 3.11, are listed in Table 3.4. The $B_{f}^{*}$ and $\Delta_{\text {shell }}^{n-1}$ values extracted using the formation cross sections from the Bass model are consistent with those obtained in the two parameter fits, and also consistent with those obtained from data for ${ }^{4} \mathrm{He}-$ induced fission. However, the corresponding values extracted using the formation cross sections from the Extra-push and Optical models are about $3 \mathrm{MeV}$ higher than those obtained using the Bass model predictions. The origin of this discrepancy will become evident in the following discussion.

The excitation functions for these ${ }^{3} \mathrm{He}$-induced fission reactions and the corresponding fits using the three different assumptions for the formation cross sections, are shown in Fig. 3.11. The fits are all excellent in the energy range where the data points were used in the fitting. In comparison with the previous two parameter fits (shown in Figs. 3.5 and 3.8), the quality of the three parameter fits (shown in Fig. 3.11) using the formation cross sections from the Extra-push and Optical models improves considerably. This is more evident in the substantially smaller $\chi^{2}$ than the corresponding $\chi^{2}$ listed Tables 3.1 and 3.2. This improvement in the fitting quality is, of course, very natural, since the three parameter fits are more flexible than the two parameter fits. On the other hand, the improvement in the quality of the fit seems to come at the expense of the consistency in the extracted parameters.

The fission excitation functions for the nuclei ${ }^{185,186,187,189} \mathrm{Os}$ produced in the ${ }^{3} \mathrm{He}$-induced reactions on $\mathrm{W}$ targets, and the corresponding three parameter 


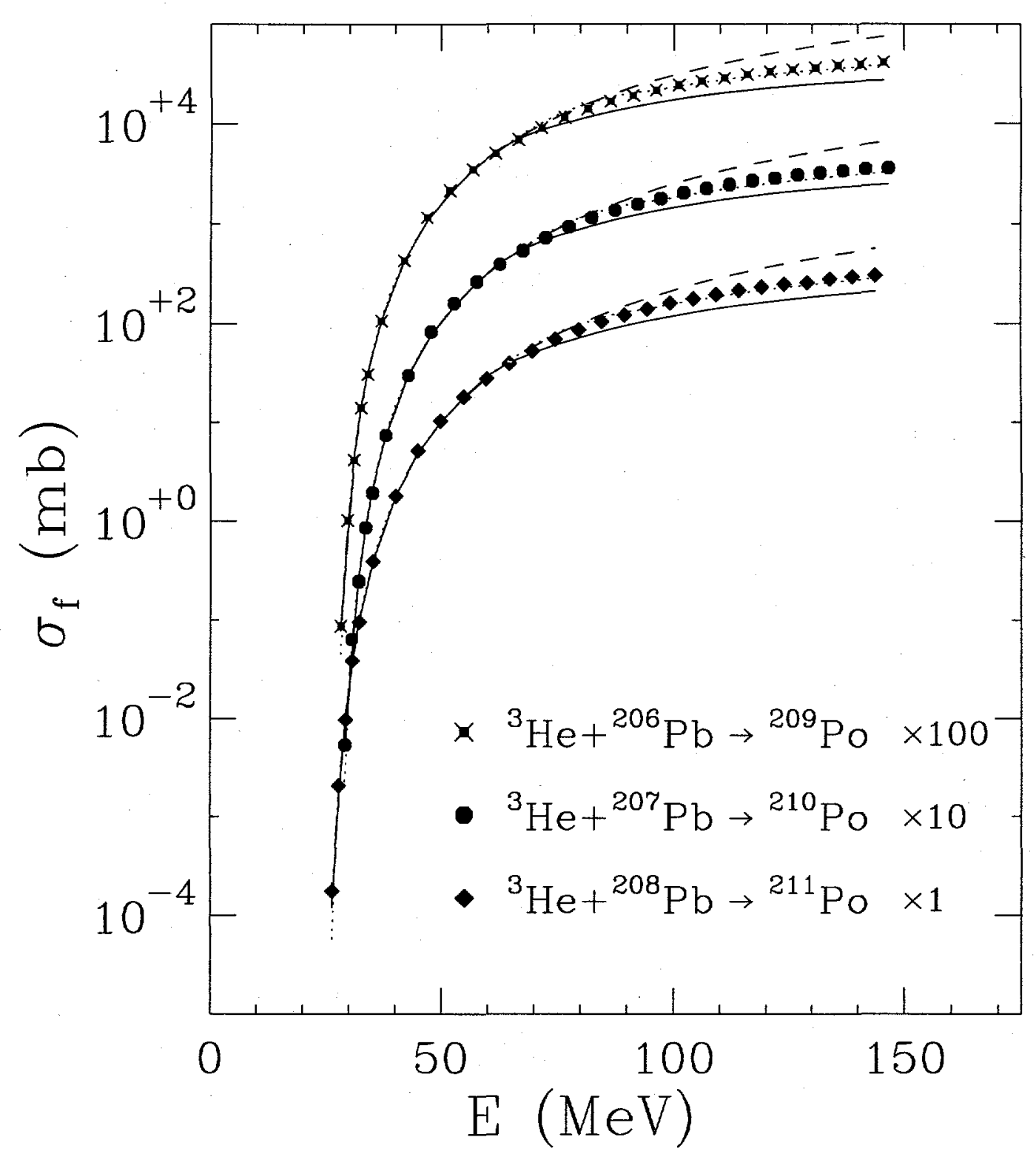

Figure 3.11: The fission excitation functions for the nuclei ${ }^{209,210,211}$ Po produced in the reactions ${ }^{3} \mathrm{He}+{ }^{206,207,208} \mathrm{~Pb}$, were fitted with the statistical formalism, with $B_{f}^{*}, \Delta_{\text {shell }}^{n-1}$, and $a_{f} / a_{n}$ as the three free parameters. The solid lines, the dotted lines and the dashed lines represent fits using the formation cross sections of the fissioning nuclei calculated by the Bass, the Extra-push and the Optical models, respectively. The fits were attempted for only the low energy portion of the fission excitation functions. The highest energy of the data segment used in the fitting is indicated by $E_{H}$ in Table 3.4. The fits were extended to the full range of the experimental data, using the extracted parameters $\left(B_{f}^{*}, \Delta_{\text {shell }}^{n-1}\right.$, and $\left.a_{f} / a_{n}\right)$ which are listed in "Table 3.4. The number to the right of each legend indicates the factor by which the corresponding excitation function is multiplied for visual clarity. 
Table 3.4: The effective fission barriers $B_{f}^{*}$, the shell effects $\Delta_{\text {shell }}^{n-1}$ of the residual nuclei after neutron emission, and the ratios $a_{f} / a_{n}$, extracted for polonium isotopes produced in the ${ }^{3} \mathrm{He}$-induced reactions by fitting the low energy portion of the measured fission excitation functions. The data and the corresponding fits are shown in Fig. 3.11. The fits were performed with three different assumptions for the formation cross sections $\sigma_{0}$ of the fissioning compound nuclei $(\mathrm{CN})$ : the Bass, the Extra-push and the Optical models. $a_{n}=A / 8\left(\mathrm{MeV}^{-1}\right)$ was assumed. $E_{H}(\mathrm{MeV})$ indicates the highest excitation energy of the data points up to which the fits were attempted.

\begin{tabular}{lcc|ccccc|l}
\hline \hline Reaction & \multirow{2}{*}{$\mathrm{CN}$} & $E_{H}$ & $B_{f}^{*}$ & $\Delta_{\text {shell }}^{n-1}$ & $B_{f}^{*}-\Delta_{\text {shell }}^{n-1}$ & $a_{f} / a_{n}$ & \multicolumn{1}{c}{$\chi^{2}$} & \multicolumn{1}{|l}{$\sigma_{0}$} \\
\hline \multirow{3}{*}{${ }^{3} \mathrm{He}+{ }^{206} \mathrm{~Pb}$} & ${ }^{209} \mathrm{Po}$ & 66.6 & 22.89 & 10.53 & 12.36 & 1.078 & 16.4 & Bass \\
& & & 25.90 & 14.45 & 11.45 & 1.047 & 15.5 & Extra-push \\
& & & 25.87 & 14.23 & 11.64 & 1.032 & 9.2 & Optical \\
\hline \multirow{3}{*}{${ }^{3} \mathrm{He}+{ }^{207} \mathrm{~Pb}$} & \multirow{2}{*}{${ }^{210} \mathrm{Po}$} & 67.6 & 23.82 & 10.44 & 13.38 & 1.078 & 19.0 & Bass \\
& & & 26.91 & 14.54 & 12.37 & 1.045 & 14.0 & Extra-push \\
& & & 26.69 & 14.06 & 12.63 & 1.032 & 9.0 & Optical \\
\hline \multirow{3}{*}{${ }^{3} \mathrm{He}+{ }^{208} \mathrm{~Pb}$} & \multirow{2}{*}{${ }^{211} \mathrm{Po}$} & 64.8 & 22.05 & 11.56 & 10.49 & 1.075 & 12.1 & Bass \\
& & & 24.40 & 14.75 & 9.65 & 1.045 & 14.7 & Extra-push \\
\hline \hline
\end{tabular}

fits, are shown in Fig. 3.12. The fits, still using the same three assumptions for the formation cross sections, are all excellent in the energy range fitted. The $B_{f}^{*}$ and $\Delta_{\text {shell }}^{n-1}$ values extracted using the different formation cross sections, however, lose their consistency. The values for $B_{f}^{*}, \Delta_{\text {shell }}^{n-1}$, and $a_{f} / a_{n}$ extracted from the three parameter fits, are listed in Table 3.5. One observes again that, the $B_{f}^{*}$ and $\Delta_{\text {shell }}^{n-1}$ extracted using the formation cross sections from the Bass model are consistent with those obtained in the two parameter fits. The $B_{f}^{*}$ and $\Delta_{\text {shell }}^{n-1}$ extracted using the formation cross sections from the Extra-push and Optical models are, however, several $\mathrm{MeV}$ higher than those obtained using the formation cross sections from the Bass model. As observed previously in the two parameter fits, the $a_{f} / a_{n}$ values extracted 
from the three parameter fits seem to be the same for all the nuclei under consideration (see Tables 3.3, 3.4 and 3.5).

Now the question is: why, in the case of the ${ }^{4} \mathrm{He}$-induced fission, the three parameter fits allow one to determine the fission barrier $B_{f}^{*}$ and the shell effect $\Delta_{\text {shell }}^{n-1}$, simultaneously and accurately, regardless of the large uncertainties in the formation cross sections of the fissioning nuclei, whereas in the case of the ${ }^{3} \mathrm{He}$-induced fission the three parameter fits seem to be too flexible to give consistent values for $B_{f}^{*}$ and $\Delta_{\text {shell }}^{n-1} ?$

The answer probably lies in the difference in the energy dependence of the formation cross sections of the ${ }^{3} \mathrm{He}$-induced fission and the ${ }^{4} \mathrm{He}$-induced fission. To form a fissioning nucleus, the entrance channel reaction must first overcome a fusion (Coulomb) barrier. The fusion barriers which both the entrance channel with ${ }^{3} \mathrm{He}$ and the entrance channel with ${ }^{4} \mathrm{He}$ must overcome to form the same fissioning nuclei, are of the same magnitude, since the Coulomb repulsion depends only on the charge and geometry of the approaching projectile and the target. For helium induced reactions; the fusion barrier $B_{\text {fus }}(\mathrm{MeV})$ can be estimated with the semi-empirical formula [Park 91, Vaz 84]:

$$
B_{\text {fus }}=\frac{2.88 Z_{\text {target }}}{1.18 A_{\text {target }}^{1 / 3}+4.642}
$$

where $Z_{\text {target }}$ and $A_{\text {target }}$ are the atomic number and the mass of the target nucleus, respectively. With this equation, the fusion barrier for $\mathrm{He}+\mathrm{Pb}$ is calculated to be $20.3 \mathrm{MeV}$, and the fusion barrier for $\mathrm{He}+\mathrm{W}$ to be $18.8 \mathrm{MeV}$. These fusion barriers 
are similar to the fission barriers, which are to be determined for the nuclei formed in these reactions, and which are to be determined from the fission excitation functions measured in these reactions! This must be the origin of the difficulties.

For ${ }^{4} \mathrm{He}$-induced fusion reactions in the lead region, the $Q$ values are generally smaller than zero by several $\mathrm{MeV}$. For the compound nucleus to have an excitation energy above the fission barrier, the minimum energy of the incident ${ }^{4} \mathrm{He}$ particle must be at least $|Q| \mathrm{MeV}$ greater than the fission barrier. Thus the minimum ${ }^{4} \mathrm{He}$ energy must be substantially larger than the fusion barrier. In this case, the energy dependence of the formation cross sections of the fissioning nuclei becomes insignificant, and the exact knowledge of the formation cross sections is less critical in determining the fission barriers from the measured fission excitation functions.

For ${ }^{4} \mathrm{He}+{ }^{206,207,207} \mathrm{~Pb} \rightarrow{ }^{210,211,212} \mathrm{Po}$ reactions, the $Q$ values are -5.41 , -7.59 , and $-8.95 \mathrm{MeV}$, respectively. The minimum beam energy for which the fission cross sections were measured in this work, is $32.0 \mathrm{MeV}$ (see Table 2.4), far above the fusion barrier of $20.3 \mathrm{MeV}$. At a ${ }^{4} \mathrm{He}$ incident energy of $32.0 \mathrm{MeV}$ the fissioning nuclei ${ }^{210,211,212} \mathrm{Po}$ have excitation energies of $25.98,23.80$, and $22.44 \mathrm{MeV}$, respectively, which are very close to the fission barriers of these nuclei. In fact, these lowest excitation energies are so close to the fission barriers, that one or two of the lowest energy data points had to be excluded in the fitting in order for the asymptotic expressions of the level densities (Eqs. 1.14 and 1.17) to be applicable. For ${ }^{4} \mathrm{He}$ $+{ }^{206,207,207} \mathrm{~Pb} \rightarrow{ }^{210,211,212} \mathrm{Po} \rightarrow$ fission, since the energies of the entrance channel were far above the fusion barriers in the whole range of the measured excitation 


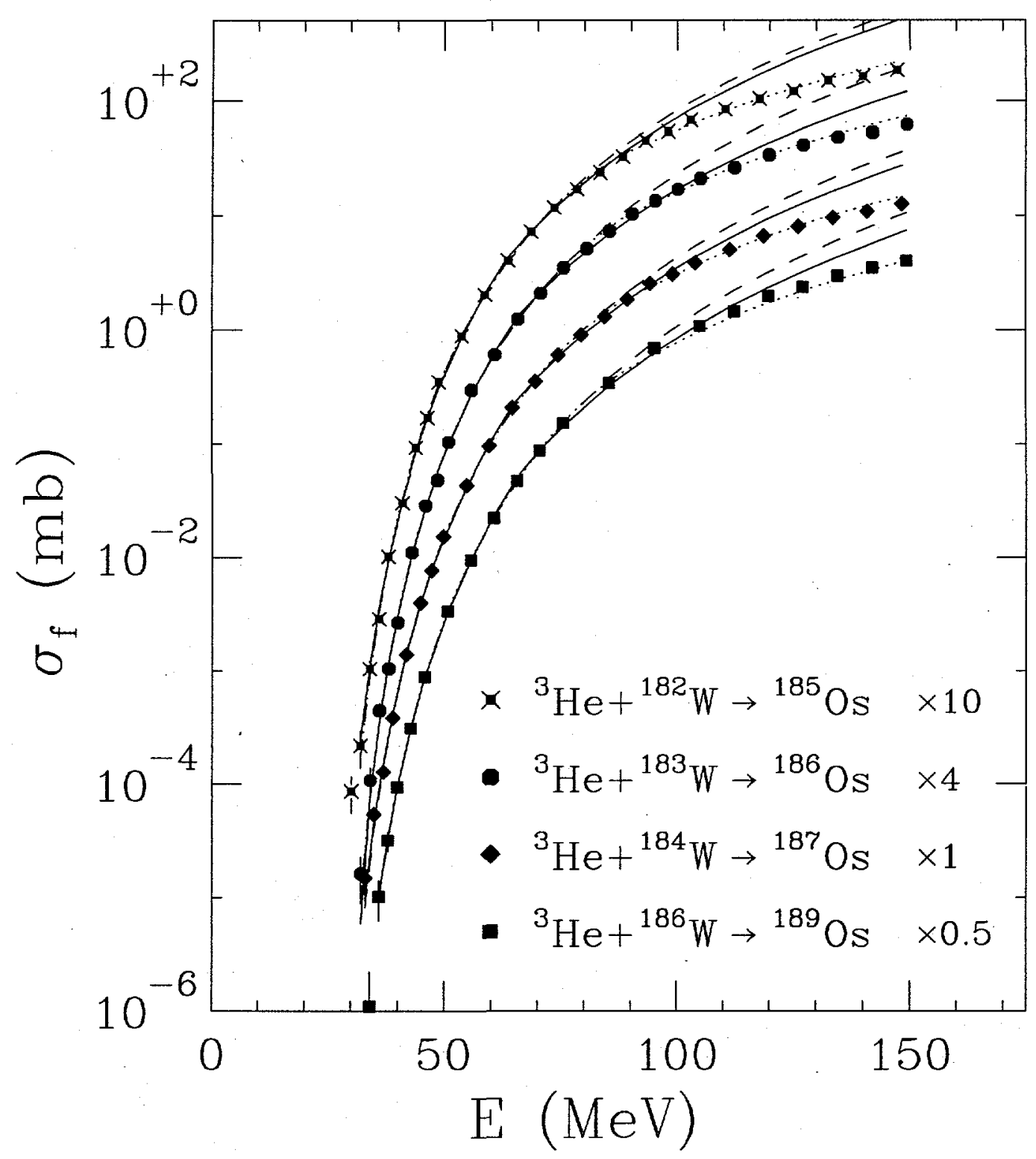

Figure 3.12: The fission excitation functions for the nuclei ${ }^{185,186,187,189}$ Os produced in the reactions ${ }^{3} \mathrm{He}+{ }^{182,183,184,186} \mathrm{~W}$, were fitted with the statistical formalism, with $B_{f}^{*}, \Delta_{\text {shell }}^{n-1}$, and $a_{f} / a_{n}$ as the three free parameters. The solid lines, the dotted lines and the dashed lines represent fits using the formation cross sections of the fissioning nuclei calculated by the Bass, the Extra-push and the Optical models, respectively. The fits were attempted for only the low energy portion of the fission excitation functions. The highest energy of the data segment used in the fitting is indicated by $E_{H}$ in Table 3.5. The fits were extended to the full range of the experimental data, using the extracted parameters $\left(B_{f}^{*}, \Delta_{\text {shell }}^{n-1}\right.$, and $\left.a_{f} / a_{n}\right)$ which are listed in Table 3.5. The number to the right of each legend indicates the factor by which the corresponding excitation function is multiplied for visual clarity. 
Table 3.5: The effective fission barriers $B_{f}^{*}$, the shell effects $\Delta_{\text {shell }}^{n-1}$ of the residual nuclei after neutron emission, and the ratios $a_{f} / a_{n}$, extracted for osmium isotopes produced in the ${ }^{3} \mathrm{He}$-induced reactions by fitting the low energy portion of the measured fission excitation functions. The data and the corresponding fits are shown in Fig. 3.12. The fits were performed with three different assumptions for the formation cross sections $\sigma_{0}$ of the fissioning compound nuclei $(\mathrm{CN})$ : the Bass, the Extra-push and the Optical models. $a_{n}=A / 8\left(\mathrm{MeV}^{-1}\right)$ was assumed. $E_{H}(\mathrm{MeV})$ indicates the highest excitation energy of the data points up to which the fits were attempted.

\begin{tabular}{|c|c|c|c|c|c|c|c|c|}
\hline Reaction & $\mathrm{CN}$ & $E_{H}$ & $B_{f}^{*}$ & $\Delta_{\text {shell }}^{n-1}$ & $B_{f}^{*}-\Delta_{\text {shell }}^{n-1}$ & $a_{f} / a_{n}$ & $\chi^{2}$ & $\sigma_{0}$ \\
\hline \multirow{3}{*}{${ }^{3} \mathrm{He}+{ }^{182} \mathrm{~W}$} & \multirow{3}{*}{${ }^{185} \mathrm{Os}$} & \multirow{3}{*}{73.4} & 20.91 & 0.04 & 20.87 & 1.108 & 9.7 & Bass \\
\hline & & & 25.38 & 7.42 & 17.96 & 1.042 & 4.5 & Extra-push \\
\hline & & & 25.00 & 6.64 & 18.36 & 1.031 & 4.2 & Optical \\
\hline \multirow{3}{*}{${ }^{3} \mathrm{He}+{ }^{183} \mathrm{~W}$} & \multirow{3}{*}{${ }^{186} \mathrm{Os}$} & \multirow{3}{*}{75.5} & 23.27 & 0.95 & 22.32 & 1.096 & 13.4 & Bass \\
\hline & & & 26.22 & 6.00 & 20.22 & 1.044 & 10.7 & Extra-push \\
\hline & & & 25.82 & 5.20 & 20.62 & 1.033 & 10.3 & Optical \\
\hline \multirow{3}{*}{${ }^{3} \mathrm{He}+{ }^{184} \mathrm{~W}$} & \multirow{3}{*}{${ }^{187} \mathrm{Os}$} & \multirow{3}{*}{74.4} & 21.96 & 0.76 & 21.20 & 1.101 & 6.0 & Bass \\
\hline & & & 25.18 & 6.33 & 18.85 & 1.045 & 4.9 & Extra-push \\
\hline & & & 25.28 & 6.26 & 19.02 & 1.030 & 4.3 & Optical \\
\hline \multirow{3}{*}{${ }^{3} \mathrm{He}+{ }^{186} \mathrm{~W}$} & \multirow{3}{*}{${ }^{189} \mathrm{Os}$} & \multirow{3}{*}{75.4} & 22.52 & 0.77 & 21.75 & 1.096 & 3.7 & Bass \\
\hline & & & 25.65 & 6.27 & 19.38 & 1.041 & 0.4 & Extra-push \\
\hline & & & 25.46 & 5.75 & 19.71 & 1.029 & 0.5 & Optical \\
\hline
\end{tabular}

functions, the energy dependence of the formation cross sections of ${ }^{210,211,212} \mathrm{Po}$ becomes insignificant. The fission barriers $B_{f}^{*}$ and the shell effects $\left.\Delta_{\text {shell }}^{n-1}\right)$ are therefore expected to be well determined from the fission excitation functions, even given the large uncertainties in the formation cross sections. It is indeed so, as shown in this and the previous sections.

For ${ }^{3} \mathrm{He}$-induced fusion reactions in the lead region, the $Q$ values are, however, greater than zero by several $\mathrm{MeV}$ or more. Since the fusion barriers for these reactions are close to the fission barriers, the excitation energies of the fissioning nuclei, formed with ${ }^{3} \mathrm{He}$ energies near the fusion barrier, may still be far above the 
corresponding fission barriers. In this case, on the one hand, it is not feasible, to measure the fission excitation functions very close to the fission barriers, and on the other hand, the fission cross sections depend increasingly on the formation cross sections as the incident energy approaches the fusion barriers. To determine fission barriers and shell effects accurately by fitting the ${ }^{3} \mathrm{He}$-induced fission excitation functions, better knowledge of the formation cross sections is thus required.

For reactions ${ }^{3} \mathrm{He}+{ }^{206,207,208} \mathrm{~Pb} \rightarrow{ }^{209,210,211} \mathrm{Po}$, the $Q$ values are +7.51 , +8.43 , and $+5.62 \mathrm{MeV}$, respectively. The minimum ${ }^{3} \mathrm{He}$ energy for which the fission cross sections were measured in this work, was $21.0 \mathrm{MeV}$ (see Table 2.3), barely enough to overcome the fusion barrier of $20.3 \mathrm{MeV}$. The incident ${ }^{3} \mathrm{He}$ particles of 21.0 $\mathrm{MeV}$ resulted in excitation energies of the formed fissioning nuclei ${ }^{209,210,211} \mathrm{Po}$ of $28.21,29.13$, and $26.31 \mathrm{MeV}$, respectively, which are substantially above the fission barriers of these nuclei. At the lowest bombarding energies near the fusion barriers, the energy dependence of the fusion cross sections becomes significant. Thus both the formation cross sections and the properties $\left(B_{f}^{*}, \Delta_{\text {shell }}^{n-1}, a_{f} / a_{n}\right)$ of these fissioning nuclei are important in determining the fission cross sections. Since the formation cross sections are not well known, the fission barriers $B_{f}^{*}$ and the shell effects $\Delta_{\text {shell }}^{n-1}$ extracted from the fits using the different assumptions for the fusion cross sections may suffer, not surprisingly, from significant variations originating from uncertainties in the fusion cross sections. This explains the discrepancy in the values of $B_{f}^{*}$ and $\Delta_{\text {shell }}^{n-1}$ extracted using the different fusion cross sections (see Table 3.4). The fact that the $B_{f}^{*}$ and $\Delta_{\text {shell }}^{n-1}$ extracted using the fusion cross sections from the Bass model 
are consistent with those obtained from the two parameter fits, may suggest that the fusion cross sections predicted by the Bass model are closer to the correct values.

For ${ }^{3} \mathrm{He}+{ }^{182,183,184,186} \mathrm{~W} \rightarrow{ }^{185,186,187,189} \mathrm{Os}$ reactions, the $Q$ values are even larger: $+9.49,+11.57,+10.45$, and $+11.41 \mathrm{MeV}$, respectively. The minimum ${ }^{3} \mathrm{He}$ energy for which the fission cross sections were measured for these systems, was 21.0 $\mathrm{MeV}$ (see Table 2.2), which is just above the fusion barrier of $18.8 \mathrm{MeV}$. At an incident energy of $21.0 \mathrm{MeV}$, the fissioning nuclei ${ }^{185,186,187,189} \mathrm{Os}$ were formed with excitation energies of $30.15,32.22,31.11$, and $32.07 \mathrm{MeV}$, respectively, which are far above the fission barriers of these nuclei. It is thus expected that the formation cross sections at the lowest energies play even a larger role in determining the fission cross sections than seen in the ${ }^{3} \mathrm{He}+\mathrm{Pb}$ reactions. The fission barriers $B_{f}^{*}$ and the shell effects $\Delta_{\text {shell }}^{n-1}$ extracted from the fits using the different assumptions for the formation cross section may suffer, as in the case of the ${ }^{3} \mathrm{He}$-induced fission on $\mathrm{Pb}$ targets, substantial variations resulting from the lack of knowledge of the formation cross sections. This is evident in the large discrepancies in $B_{f}^{*}$ and $\Delta_{\text {shell }}^{n-1}$ extracted using the three different assumptions for the formation cross sections (see Table 3.5). Here again, the extracted $B_{f}^{*}$ and $\Delta_{\text {shell }}^{n-1}$ using the formation cross sections from the Bass model are consistent with those obtained from the two parameter fits.

At high energies, the following correlation presents itself (see Eqs. 1.12, 1.14 and 1.17):

$$
\frac{\Gamma_{f}}{\Gamma_{\text {total }}} \approx \frac{\Gamma_{f}}{\Gamma_{n}} \sim \frac{\rho_{s}\left(E-B_{f}^{*}-E_{r}^{s}\right)}{\rho_{n}\left(E-B_{n}-\Delta_{\text {shell }}^{n-1}-E_{r}^{g s}\right)}
$$




$$
\begin{aligned}
& \approx \frac{\rho_{s}\left(E-\left(B_{f}^{*}-\frac{T_{s}}{T_{n}} \Delta_{\text {shell }}^{n-1}\right)-E_{r}^{s}\right)}{\rho_{n}\left(E-B_{n}-E_{r}^{g s}\right)} \\
& \approx \frac{\rho_{s}\left(E-\left(B_{f}^{*}-\Delta_{\text {shell }}^{n-1}\right)-E_{r}^{s}\right)}{\rho_{n}\left(E-B_{n}-E_{r}^{g s}\right)} .
\end{aligned}
$$

In other words, at sufficiently high excitation energies $\left(T_{s} \approx T_{n}\right)$, it is $\left(B_{f}^{*}-\Delta_{\text {shell }}^{n-1}\right)$, the difference between the fission barrier and the shell effect, that determines the fission probabilities. In the cases where $B_{f}^{*}$ is similar to $\left(B_{n}+\Delta_{\text {shell }}^{n-1}\right)$, such as for the Po isotopes, $T_{s} \approx T_{n}$ at moderate or even lower energies, and $\left(B_{f}^{*}-\Delta_{\text {shell }}^{n-1}\right)$ is thus expected to be the relevant quantity in determining the fission probabilities over a relatively wide range of the excitation energies. The correlation presented in Eq. 3.5 may cause problems when one tries to extract both the fission barriers and shell effects simultaneously from the fission excitation functions which are not measured sufficiently close to the fission barriers.

As seen earlier, the minimum excitation energies of Os and Po nuclei produced in the ${ }^{3} \mathrm{He}$-induced reactions are substantially greater than the corresponding fission barriers, due to the high fusion barriers and the large positive $Q$ values. In such cases where the fission excitation functions were not measured sufficiently close to the fission barriers, the simultaneous extraction of the fission barriers $B_{f}^{*}$ and the shell effects $\Delta_{\text {shell }}^{n-1}$ may be difficult, since the fission probabilities are more sensitive to $\left(B_{f}^{*}-\Delta_{\text {shell }}^{n-1}\right)$ rather than $B_{f}^{*}$ and $\Delta_{\text {shell }}^{n-1}$ individually. This implies that, the $\chi^{2}$ of the three parameter fits is rather flat in the three parameter space $\left(B_{f}^{*}, \Delta_{\text {shell }}^{n-1}\right.$ and $\left.a_{f} / a_{n}\right)$ along a valley of roughly constant $\left(B_{f}^{*}-\Delta_{\text {shell }}^{n-1}\right)$. In fact, if one takes the $B_{f}^{*}$ and $\Delta_{\text {shell }}^{n-1}$ from the best fit and shifts both parameters up or down by $1-2 \mathrm{MeV}$, the 
fission excitation functions re-calculated with the shifted parameters look roughly the same as the original fit. It should not be surprising, then, that the process of minimization of $\chi^{2}$ in the three parameter fits experiences some difficulties when trying to converge.

It is instructive to examine the values of $\left(B_{f}^{*}-\Delta_{\text {shell }}^{n-1}\right)$ obtained by taking the difference of $B_{f}^{*}$ and $\Delta_{\text {shell }}^{n-1}$ extracted from the three parameter fits. These values are listed in Tables 3.3, 3.4 and 3.5. Both the $B_{f}^{*}$ and the $\Delta_{\text {shell }}^{n-1}$ values extracted using the different formation cross sections deviate quite substantially for the Os and Po isotopes produced in the ${ }^{3} \mathrm{He}$-induced reactions. The $\left(B_{f}^{*}-\Delta_{\text {shell }}^{n-1}\right)$ values, however, are more or less consistent, in spite of the large difference in the formation cross sections used in the fitting (see Table 3.4 and 3.5$)$. These $\left(B_{f}^{*}-\Delta_{\text {shell }}^{n-1}\right)$ values are also consistent with the corresponding values obtained from the two parameter fits.

The problem presented in Eq. 3.5 can be ameliorated, or may even be eliminated, by measuring the fission excitation functions closer to the fission barriers. This can be achieved for the ${ }^{4} \mathrm{He}$-induced fission, taking advantage of the negative $Q$ values of the entrance channel reactions. The large negative $Q$ values of the ${ }^{4} \mathrm{He}$-induced reactions on $\mathrm{Pb}$ targets not only allow the measurements of the fission excitation functions very close to the fission barriers, but also elevate the minimum ${ }^{4} \mathrm{He}$ energy required to substantially above the fusion barriers, thus diminishing the role of the fusion cross sections. This is why we were able to determine, simultaneously and accurately, both $B_{f}^{*}$ and $\Delta_{\text {shell }}^{n-1}$ for Po isotopes from the excitation functions of the ${ }^{4} \mathrm{He}$-induced fission, without good knowledge of the fusion cross sections (see 
Table 3.3).

When it becomes unachievable to measure the fission excitation functions very close to the fission barriers due to physical or technical reasons, the simultaneous determination of both $B_{f}^{*}$ and $\Delta_{\text {shell }}^{n-1}$ is difficult, and it may require good knowledge of the fusion cross sections which are not yet available. If one of the two parameters $B_{f}^{*}$ and $\Delta_{\text {shell }}^{n-1}$ can be fixed somehow, the ambiguity presented by the difference $\left(B_{f}^{*}-\right.$ $\left.\Delta_{\text {shell }}^{n-1}\right)$ in Eq. 3.5 is thus eliminated, and the other parameter can then be determined unequivocally. This has been shown in Sections 3.2 and 3.3 .

\subsection{From Fission Cross Sections to Fusion Cross Sections}

The fission probabilities of the compound nuclei of the same isotope but produced in different entrance channel reactions are expected to be, with a small correction for angular momentum effects, the same at the same excitation energies. This provides a mean to compare the fusion cross sections of different entrance channels, given the fission cross sections. In particular, if both the fission excitation functions of the same nucleus but produced in two different entrance channels are known, then the fusion cross section of one entrance channel can be determined relative to the fusion cross section of the other channel.

For nuclei ${ }^{210} \mathrm{Po}$ and ${ }^{211} \mathrm{Po}$, we have measured their fission excitation functions with both the ${ }^{3} \mathrm{He}$ - and ${ }^{4} \mathrm{He}$-induced fission. The fusion cross sections of the ${ }^{3} \mathrm{He}$-induced reactions can thus be extracted relative to the fusion cross sections of 
the ${ }^{4} \mathrm{He}$-induced reactions. The method is as follows. Let $\sigma_{f}, \sigma_{0}$ and $P_{f}$ be the fission cross section, the fusion cross section and the fission probability, respectively, for the same fissioning nucleus, then

$$
\frac{\sigma_{f}\left({ }^{3} \mathrm{He}, E\right)}{\sigma_{f}\left({ }^{4} \mathrm{He}, E^{\prime}\right)} \approx \frac{\sigma_{0}\left({ }^{3} \mathrm{He}, E\right)}{\sigma_{0}\left({ }^{4} \mathrm{He}, E^{\prime}\right)} \frac{P_{f}\left({ }^{3} \mathrm{He}, E-\left\langle E_{r}\right\rangle\right)}{P_{f}\left({ }^{4} \mathrm{He}, E^{\prime}-\left\langle E_{r}^{\prime}\right\rangle\right)} \approx \frac{\sigma_{0}\left({ }^{3} \mathrm{He}, E\right)}{\sigma_{0}\left({ }^{4} \mathrm{He}, E^{\prime}\right)},
$$

where $E-\left\langle E_{r}\right\rangle=E^{\prime}-\left\langle E_{r}^{\prime}\right\rangle ; E$ and $E^{\prime}$ are the excitation energies; $\left\langle E_{r}\right\rangle$ and $\left\langle E_{r}^{\prime}\right\rangle$ denote the average rotational energies. In Eq. 3.6, the different entrance channels ( ${ }^{3} \mathrm{He}$ - and ${ }^{4} \mathrm{He}-$ induced) leading to the same fissioning nucleus are denoted explicitly.

As seen in Section 3.4, for the compound nucleus $\left({ }^{210} \mathrm{Po}\right.$ or $\left.{ }^{211} \mathrm{Po}\right)$ to be formed with the same excitation energy, the fusion reaction with ${ }^{4} \mathrm{He}$ requires beam energy greater by $\sim 13.5 \mathrm{MeV}$ than that the fusion reaction with ${ }^{3} \mathrm{He}$ requires, due to the difference in the $Q$-values. For the excitation energy range over which the fission excitation functions were measured, the fusion reaction with ${ }^{4} \mathrm{He}$ always occurs at energies far above the fusion barrier, whereas the fusion reaction with ${ }^{3} \mathrm{He}$ may occur at energies very close to the fusion barrier. Therefore, the fusion cross section $\sigma_{0}$ for the ${ }^{3} \mathrm{He}$-induced reaction near the fusion barrier where $\sigma_{0}$ changes rapidly with energy, can thus be deterimined relative to the fusion cross section for the ${ }^{4} \mathrm{He}$ induced reaction in the region where $\sigma_{0}$ depends on energy rather weakly. This makes it a worthwhile exercise to obtain in the prescribed manner the fusion cross sections for the ${ }^{3} \mathrm{He}$-induced reactions, even though the reference cross sections - the fusion cross sections of the ${ }^{4} \mathrm{He}-$ induced reactions - are also not well known.

Taking the fusion cross section for the ${ }^{4} \mathrm{He}$-induced reaction to be the Bass 


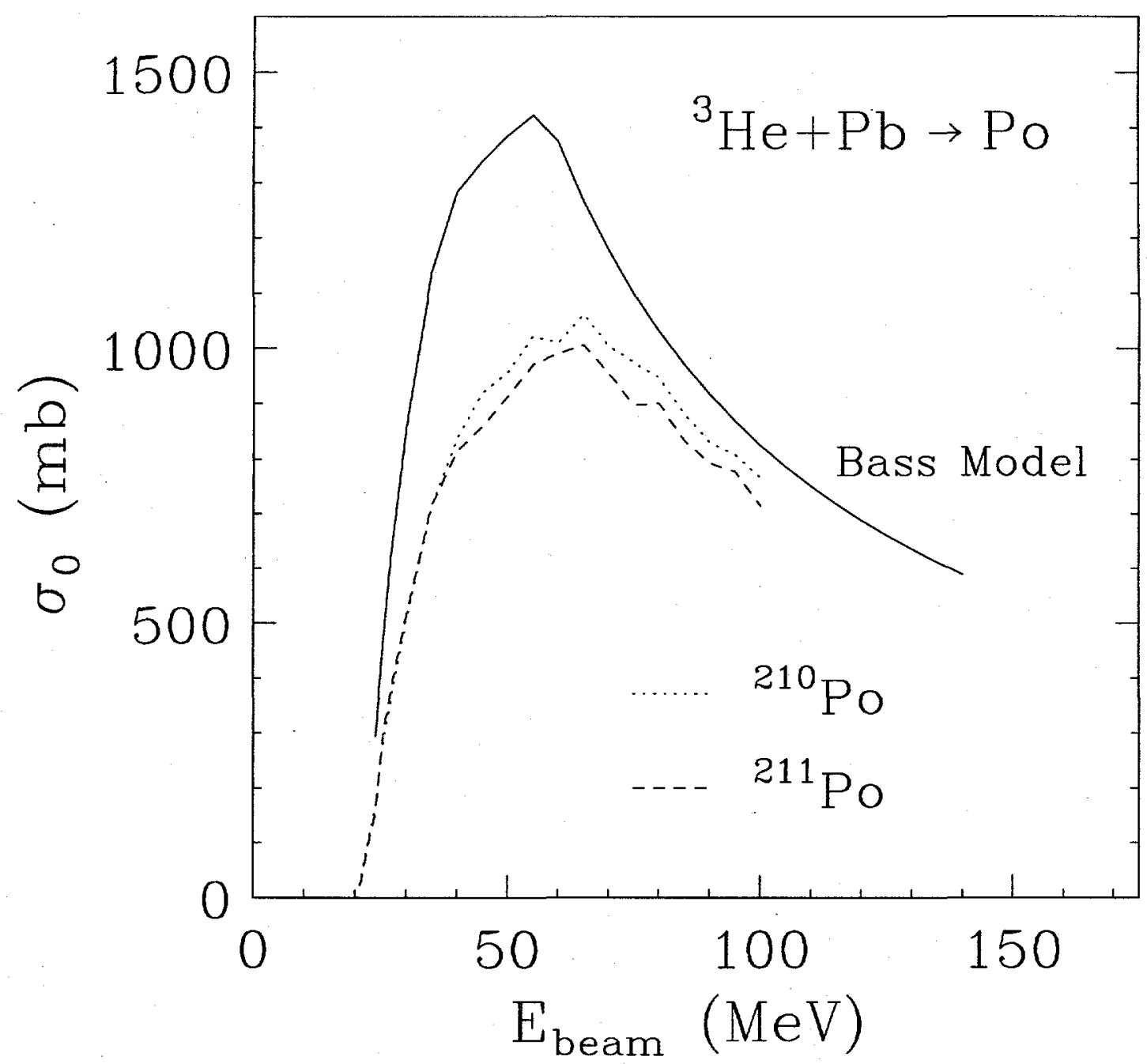

Figure 3.13: The fusion cross sections for the ${ }^{3} \mathrm{He}+\mathrm{Pb}$ reactions obtained by comparing the fission cross sections of the same compound nuclei $\left({ }^{210} \mathrm{Po}\right.$ or $\left.{ }^{211} \mathrm{Po}\right)$ produced in both the ${ }^{3} \mathrm{He}$ - and ${ }^{4} \mathrm{He}$-induced reactions, given the fusion cross sections for the ${ }^{4} \mathrm{He}$ entrance channels as the Bass model prediction. For comparison, the fusion cross section calculated with the Bass model for the ${ }^{3} \mathrm{He}+{ }^{207} \mathrm{~Pb}$ reaction is also shown. 
model prediction (see Fig. 3.3), the fusion cross section for the ${ }^{3} \mathrm{He}$-induced reaction which leads to the same compound nucleus $\left({ }^{210} \mathrm{Po}\right.$ or $\left.{ }^{211} \mathrm{Po}\right)$ can be obtained with Eq. 3.6. The results are shown in Fig. 3.13. For comparison, the fusion cross section calculated with the Bass model for the ${ }^{3} \mathrm{He}+{ }^{207} \mathrm{~Pb}$ reaction is also shown. It is interesting to note that the fusion cross sections obtained this way are even lower than the Bass model prediction.

\subsection{Summary}

In this chapter, the fission excitation functions for the nuclei ${ }^{185,186,187,189} \mathrm{Os}$ produced in the ${ }^{3} \mathrm{He}+{ }^{182,183,184,186} \mathrm{~W}$ reactions, for ${ }^{209,210,211} \mathrm{Po}$ produced in the ${ }^{3} \mathrm{He}+{ }^{206,207,208} \mathrm{~Pb}$ reactions, and for ${ }^{210,211,212} \mathrm{Po}$ produced in the ${ }^{4} \mathrm{He}+{ }^{206,207,208} \mathrm{~Pb}$ reactions, have been analyzed in detail based on the statistical formalism. The analysis aimed at an accurate determination of the effective fission barriers $B_{f}^{*}$, the shell effects $\Delta_{\text {shell }}^{n-1}$ of the residual nuclei after evaporation of a neutron, and the ratio $a_{f} / a_{n}$ of the level density at the saddle point to that of the ground state.

It was demonstrated that, in the case of the ${ }^{210,211,212}$ Po isotopes formed in the ${ }^{4} \mathrm{He}$-induced reactions, $B_{f}^{*}$ and $\Delta_{\text {shell }}^{n-1}$ can be determined from the fission excitation functions, simultaneously and with high accuracy, regardless of the large uncertainties in the formation cross sections of these nuclei. This is possible because the large negative $Q$-values of the ${ }^{4} \mathrm{He}$-induced reactions allow the fission excitation functions to be measured very close to the fission barriers, with ${ }^{4} \mathrm{He}$ energies substan- 
tially above the fusion barriers, thus diminishing the importance of the formation cross sections in the fitting process.

For nuclei formed in the ${ }^{3} \mathrm{He}$-induced reactions, however, the simultaneous determination of $B_{f}^{*}$ and $\Delta_{\text {shell }}^{n-1}$ requires better knowledge of the formation cross sections of the fissioning nuclei than is now available. The large fusion barriers and the large positive $Q$ values of the ${ }^{3} \mathrm{He}$-induced reactions on $\mathrm{Pb}$ and $\mathrm{W}$ targets make it impossible to measure the fission excitation functions sufficiently close to the fission barriers. This allows, on the one hand, for the correlation represented by Eq. 3.5 to set in, and on the other hand, pushes the minimum ${ }^{3} \mathrm{He}$ energy very close to the fusion barriers, thus leaving way for the fusion cross sections to play a larger role in determining the fission cross sections. The attempt to simultaneously determine $B_{f}^{*}$ and $\Delta_{\text {shell }}^{n-1}$ therefore produced inconsistent results. However, if one of the parameters $\left(B_{f}^{*}\right.$ or $\Delta_{\text {shell }}^{n-1}$ ) can be fixed somehow, the other parameter can then be determined consistently from the fission excitation functions, even in the face of large uncertainties in the formation cross sections.

The extracted $a_{f} / a_{n}$ values, which lie between 1.0 and 1.1 , seem to be the same for all the nuclei under study. This result, if confirmed with a larger set of experimental data, may be of fundamental significance to statistical physics. Its theoretical implications are yet to be explored. 


\section{Chapter 4}

\section{Dynamic Fission Time Scale}

The precisely measured fission excitation functions for neighboring Os and Po isotopes presented in the previous chapter should contain information on the effect of a possible transient time on the fission process, since a transient time longer than the characteristic time for fission should necessarily reduce the fission probabilities. A detailed comparison of the fission probabilities of neighboring isotopes should reveal the transient time effect and may allow us to determine its magnitude. In this chapter, a new and straight-forward way to determine the upper limit of the transient time will be developed. This novel approach bypasses all of the difficulties associated with the separation of the presaddle and postsaddle particle emission. The upper limits for the transient time set with this new method are $15 \times 10^{-21}$ sec and $25 \times 10^{-21}$ sec for, respectively, the neighboring Os and Po compound nuclei produced in ${ }^{3} \mathrm{He}$-induced reactions. 


\subsection{Method}

We begin by taking the simplest assumption for transient time effects on the fission decay width, as shown in Fig. 4.1: that no fission occurs during a transient time $\tau_{D}$, and that fission assumes the transition state width beyond $\tau_{D}$. Then, the fission decay width $\Gamma_{f}^{\mathrm{tr}}$ with transient time effects can be written as

$$
\Gamma_{f}^{\mathrm{tr}}=\Gamma_{f}^{(\infty)} \int_{0}^{\infty} \zeta(t) N(t) \lambda_{C N} d t=\Gamma_{f}^{(\infty)} \int_{\tau_{D}}^{\infty} N(t) d\left(\frac{t}{\tau_{C N}}\right)
$$

where $\zeta(t)=0\left(t<\tau_{D}\right)$ and $\zeta(t)=1\left(t \geq \tau_{D}\right) ; \tau_{D}$ is the transient time; $N(t)$ is the number of the remaining compound nuclei at time $t$ (starting with 1 compound nucleus at $t=0) ; \lambda_{C N}$ is the total decay constant of the compound nucleus and $\tau_{C N}=1 / \lambda_{C N}$ is the compound nucleus lifetime, without transient time effects; $\Gamma_{f}^{(\infty)}$ denotes the expected fission width without the transient time effects, or the transition state fission width. In the following we will use $\lambda$ 's $(\tau$ 's) to refer implicitly the corresponding decay (time) constants without transient time effects unless indicated otherwise, since the transient time effects on fission rates have been nicely taken care of by the step function $\zeta(t)$.

Now consider a decay chain starting from the compound nucleus $(Z, A)$ with excitation energy $E$ :

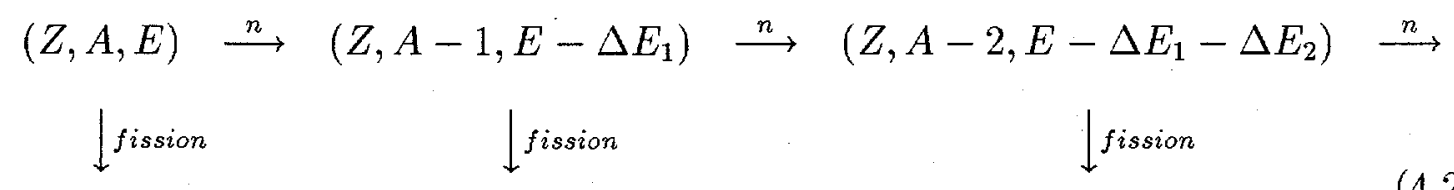

where $\Delta E_{i}(i=1,2, \cdots)$ denotes the average energy loss through the evaporation of the $i$ th neutron. $\Delta E_{i}$ can be estimated as $\left(B_{n}+2 T\right)_{i}$ with $T_{i}$ being the temperature 


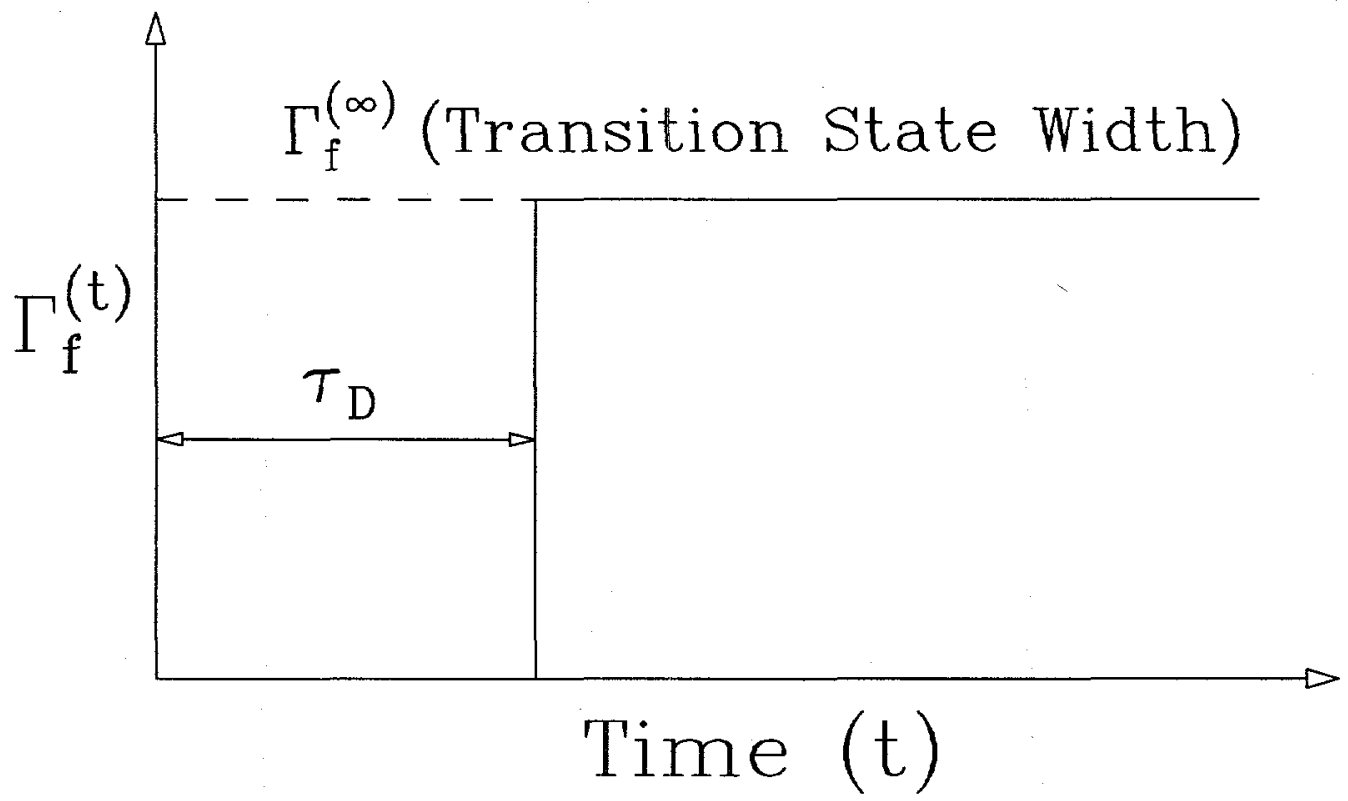

Figure 4.1: Transient time effects on the fission decay rate are taken to be a step function. $\tau_{D}$ denotes an assumed transient time.

of the residual nucleus after $i$ th neutron emission and $B_{n}$ being the corresponding neutron separation energy. $2 T_{i}$ is the average energy of evaporated neutrons of a Maxwellian energy distribution. To simplify the picture, angular momentum effects are not included. This neglect may be justified in the case of light charged particle induced fission where the angular momentum involved is generally small. Let $\lambda_{n}$, $\lambda_{f}, \lambda_{C N}\left(=\lambda_{n}+\lambda_{f}\right)$ be the neutron, fission and total decay constants of the nucleus $(Z, A, E)$, respectively; $\lambda_{n}^{\prime}, \lambda_{f}^{\prime}, \lambda_{C N}^{\prime}$ be the neutron, fission and total decay constants of the nucleus $\left(Z, A-1, E-\Delta E_{1}\right)$, respectively; and $\lambda_{n}^{\prime \prime}, \lambda_{f}^{\prime \prime} \lambda_{C N}^{\prime \prime}$ be the neutron, fission and total decay constants of the nucleus $\left(Z, A-2, E-\Delta E_{1}-\Delta E_{2}\right)$, respectively. The inverse of these decay constants defines the corresponding characteristic times: $\tau_{n}, \tau_{f}$, $\tau_{C N}, \tau_{n}^{\prime}, \tau_{f}^{\prime}, \tau_{C N}^{\prime}, \tau_{n}^{\prime \prime}, \tau_{f}^{\prime \prime}, \tau_{C N}^{\prime \prime}$. Let $P_{f}(A, Z, E)$ be the expected fission probability for the compound nucleus $(Z, A)$ with excitation energy $E$ in the limit that no transient 
time effects are present. Let us further assume that the decay clock is not reset at each step in the decay chain. Given a transient time $\tau_{D}$, the observed total fission probability $P_{f}^{t}(Z, A, E)$ can be written as

$$
P_{f}^{t}(Z, A, E)=P_{f}^{1 s t}+P_{f}^{2 n d}+P_{f}^{3 r d}+\cdots
$$

where

$$
\begin{aligned}
& P_{f}^{1 s t}=P_{f}(Z, A, E) e^{-\tau_{D} / \tau_{n}}, \\
& P_{f}^{2 n d}=P_{f}\left(Z, A-1, E-\Delta E_{1}\right)\left[\frac{\lambda_{n}}{\lambda_{C N}} e^{-\tau_{D} / \tau_{n}}+\frac{\lambda_{n}}{\lambda_{n}-\lambda_{n}^{\prime}}\left(e^{-\tau_{D} / \tau_{n}^{\prime}}-e^{-\tau_{D} / \tau_{n}}\right)\right], \text { etc. }
\end{aligned}
$$

In this equation, $P_{f}^{1 s t}, P_{f}^{2 n d}$ and $P_{f}^{3 r d}$ represent the first, second, and third chance fission probabilities expected for the nucleus $(Z, A, E)$, with a transient time $\tau_{D}$, respectively. The neutron and fission decay constants can be estimated with Eqs. 1.9 and 1.10. $P_{f}(Z, A, E), P_{f}\left(Z, A-1, E-\Delta E_{1}\right), P_{f}^{3 r d}$, and $\tau_{D}$ are, however, unknown. The non-reset of the decay clock after neutron emission should be a reasonable assumption, as can be seen in the following. Let $V(s)$ be the potential energy surface with $s$ designating the shape or deformation coordinates of a nucleus. The probability distribution $p(s)$ of the compound nucleus as a function of shape $s$ can be written as

$$
p(s) \propto \rho(E-V(s)),
$$

where $\rho(E-V(s))$ is the level density at the corresponding shape $s$. The probability distribution $p^{\prime}$ of the residual nucleus after neutron emission should be

$$
\begin{aligned}
p^{\prime} & \propto p(s) \cdot \Gamma_{n}(s) \propto \rho(E-V(s)) \cdot \frac{\rho\left(E-V(s)-B_{n}\right)}{\rho(E-V(s))} \\
& \approx \rho(E-V(s)) \cdot e^{-B_{n} / T} .
\end{aligned}
$$


In other words, the residual nucleus retains the same probability distribution profile as the mother nucleus has (Eq. 4.4). The assumption that the decay clock is not reset after neutron emission is thus more or less justified.

While Eq. 4.3 looks hopelessly insoluble, it does provide clues to an upper limit of fission transient time $\tau_{D}$. When $P_{f}^{t}(Z, A, E)$ is specified, there exists a maximum value of $\tau_{D}$ for which Eq. 4.3 can be satisified. Taking the experimental value $P_{f}^{t}\left(Z, A-2, E-\Delta E_{1}-\Delta E_{2}\right)$ as the upper limit of $P_{f}^{3 r d}$, and replacing $P_{f}^{3 r d}$ in Eq. 4.3 with this upper limit, and replacing $P_{f}\left(Z, A-1, E-\Delta E_{1}\right)$ with the the experimental value $P_{f}^{t}\left(Z, A-1, E-\Delta E_{1}\right)$, the resulting equation can then be solved for an upper limit of $\tau_{D}$. One must keep in mind, of course, that $P_{f} \in[0,1]$.

\subsection{Upper Limits of Fission Transient Times for Os and Po Isotopes}

The experimental fission probability can be determined as the ratio of the fission cross section $\sigma_{f}$ and the compound nucleus formation cross section $\sigma_{0}$. We have measured with high precision the fission cross sections for several Os and Po isotopes, and presented in chapter 2.2 the experimental data in Figs. $2.2 \& 2.3$ and Tables $2.2,2.3 \& 2.4$

The compound nucleus formation cross sections or the fusion cross sections for these reaction systems are, however, not known, and have to be estimated with certain theoretical models such as the Bass model [Bass 74], the Extra-Push model 
[Swia 82], etc.. The fusion cross sections calculated with the Bass model and the Extra-push model for the reactions ${ }^{3} \mathrm{He}+{ }^{184} \mathrm{~W},{ }^{3} \mathrm{He}+{ }^{207} \mathrm{~Pb}$ and ${ }^{4} \mathrm{He}+{ }^{207} \mathrm{~Pb}$ are shown in Figs. 3.1, 3.2 and 3.3. Also plotted in these figures are the reaction cross sections predicted by the optical model [Macf 78].

In the following analysis, we choose the fusion cross sections predicted by the Bass model to determine the fission probabilities. The reason for this choice will be discussed later on. The fission probabilities $P_{f}^{t}(Z, A, E)$ of different isotopes at different excitation energies can then be calculated accordingly as $\sigma_{f} / \sigma_{0}$.

We now try to solve for upper limits for the transient time $\tau_{D}$, for both the Os isotopes and the Po isotopes, in two ways: 1) by setting $P_{f}(Z, A, E)=1$. This will obviously result in very relaxed upper limits of $\left.\tau_{D} ; 2\right)$ by setting $P_{f}(Z, A, E)=$ $P_{f}^{t}(Z, A, E)$. This should result in more stringent, and perhaps more proper, upper limits of $\tau_{D}$.

In Fig. 4.2 we show the presaddle transient time upper limits derived for Os isotopes in the prescribed ways plotted against the excitation energy of ${ }^{187}$ Os. One sees that the upper limit obtained by setting $P_{f}(Z, A, E)=P_{f}^{t}(Z, A, E)$ does not change substantially as the energy changes. The very relaxed upper limit derived by setting $P_{f}(Z, A, E)=1$, on the other hand, increases as the lifetime of the compound nucleus increases (decreasing in energy). However, the upper limits derived both ways converge at the highest excitation energies where the compound nucleus lifetime is short. The upper limit of the transient time can therefore be set at $15 \times 10^{-21}$ sec.

The upper limits for the transient times for Po isotopes are plotted against 


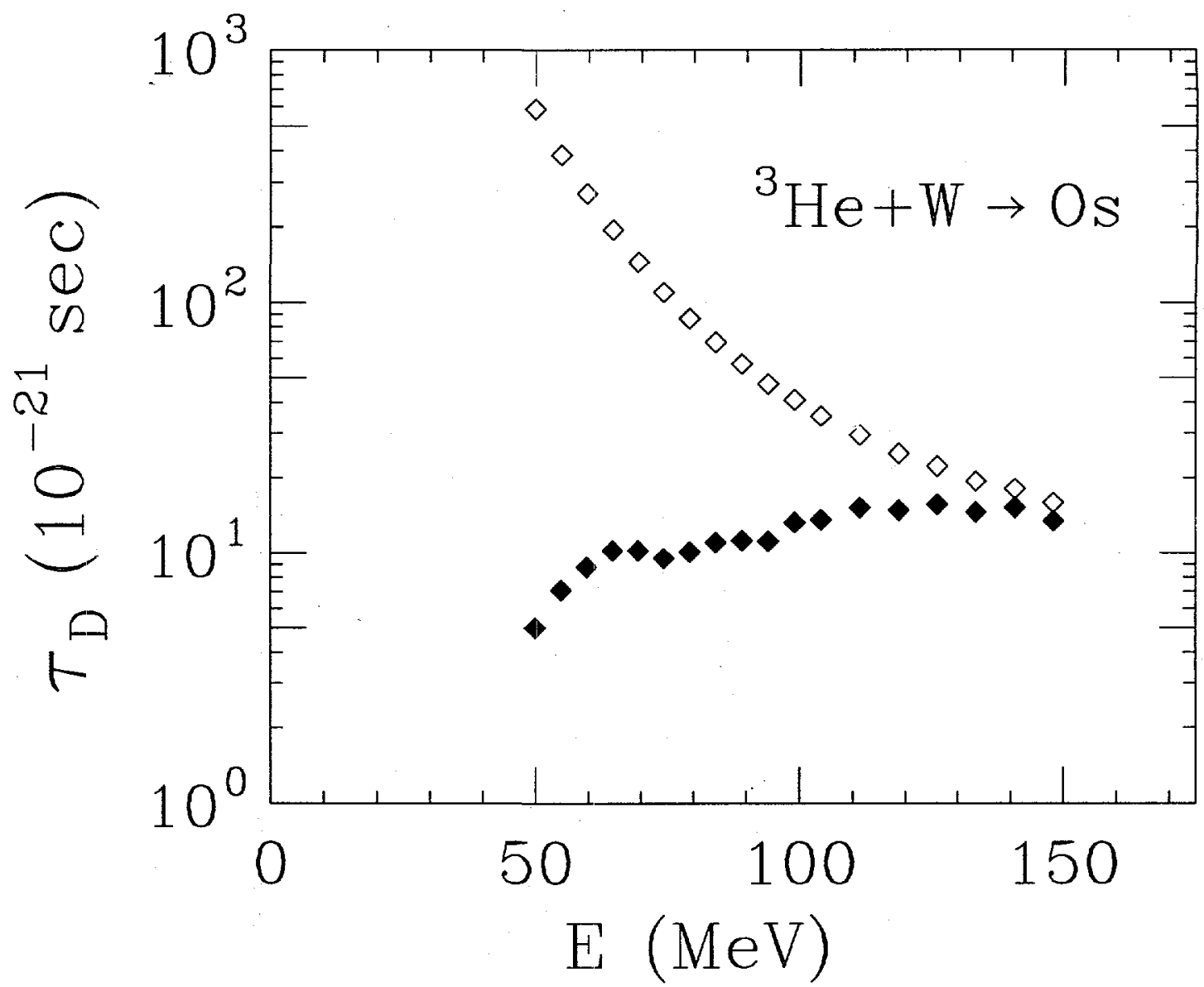

Figure 4.2: The upper limits of the transient times determined from the fission probabilities of neighboring isotopes ${ }^{185,186,187} \mathrm{Os}$ produced in ${ }^{3} \mathrm{He}+\mathrm{W}$ reactions are plotted at different excitation energies. The abscissa represents the excitation energy of ${ }^{187} \mathrm{Os}$. The very relaxed upper limits (open symbol) are obtained by assuming $P_{f}(Z, A, E)=1$, and the more proper upper limits (solid symbol) are obtained by assuming $P_{f}(Z, A, E)=P_{f}^{t}(Z, A, E)$. See text. 


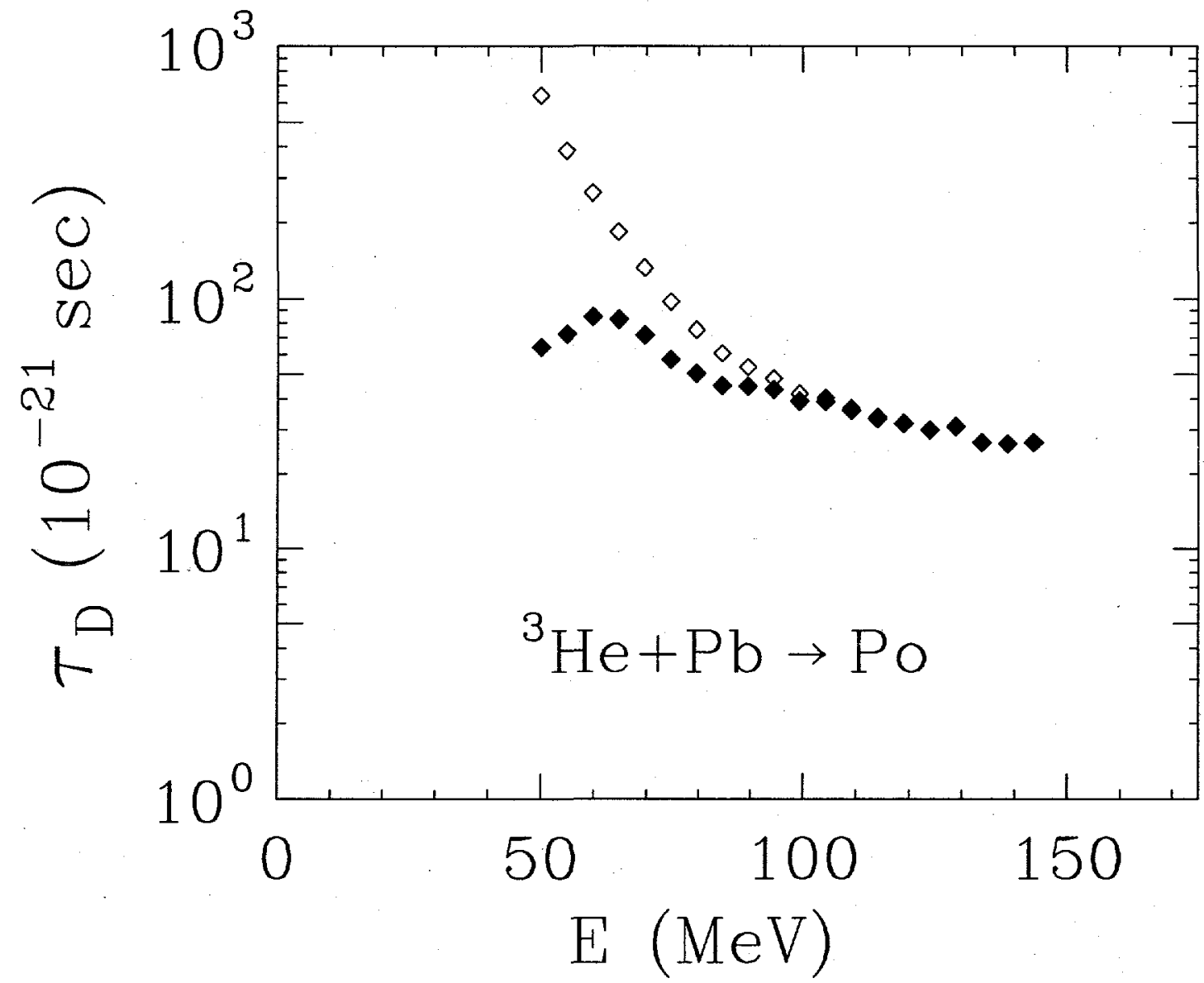

Figure 4.3: The upper limits of the transient times determined from the fission probabilities of neighboring isotopes ${ }^{209,210,211} \mathrm{Po}$ produced in ${ }^{3} \mathrm{He}+\mathrm{Pb}$ reactions are plotted at different excitation energies. The abscissa represents the excitation energy of ${ }^{211} \mathrm{Po}$. The very relaxed upper limits (open symbol) are obtained by assuming $P_{f}(A, E)=1$, and the more proper upper limits (solid symbol) are obtained by assuming $P_{f}(A, E)=P_{f}^{t}(A, E)$. See text. 
the excitation energy of ${ }^{211} \mathrm{Po}$ in Fig. 4.3. As in the case of Os, the very relaxed upper limit derived by setting $P_{f}(Z, A, E)=1$ and the upper limit obtained by setting $P_{f}(Z, A, E)=P_{f}^{t}(Z, A, E)$ converge at about $25 \times 10^{-21} \mathrm{sec}$ at the highest excitation energies. For Po isotopes, the transient upper limit can then be set at $25 \times 10^{-21} \mathrm{sec}$.

The approach presented above is sensitive when the lifetimes of the compound nuclei under consideration are sufficiently short. If the lifetimes of the nuclei are too long, the upper limit set this way may become so relaxed as to be irrelevant, as can be seen in Fig. 4.3 for Po.

This approach also hinges on the assumption that the observed total fission probability (all chances included) $P_{f}^{t}\left(Z, A-2, E-\Delta E_{1}-\Delta E_{2}\right)$ can be taken as the upper limit of $P_{f}^{3 r d}+\cdots$. While this assumption may not be logically sound, it is most likely the case, and justifiable at the energy regime where transient time effects on fission probability are expected to be very small for nucleus $\left(Z, A-2, E-\Delta E_{1}-\Delta E_{2}\right)$. For example, when $E\left({ }^{187} \mathrm{Os}\right)<80 \mathrm{MeV}, \tau_{C N}\left({ }^{185} \mathrm{Os}, E-\Delta E_{1}-\Delta E_{2}\right)>38 \times 10^{-21}$ sec and $\tau_{D}<10 \times 10^{-21} \sec$ (see Fig. 4.2), whereas $P_{f}^{t}\left({ }^{185} \mathrm{Os}, E-\Delta E_{1}-\Delta E_{2}\right)<16 \%$ of $P_{f}^{t}\left({ }^{187} \mathrm{Os}, E\right)$. In this case the assumption is clearly justified.

The fusion cross sections $\sigma_{0}$ (see Fig. 3.1, 3.2 and 3.3), which are calculated with the Bass model [Bass 74] and used to determine the total fission probability $P_{f}^{t}$ in the current analysis, represent a major uncertainty. There is little direct experimental evidence in the energy regime of particular interest to this work $(>70$ $\mathrm{MeV}$ ) that can be used for us to judge the correctness of the Bass Model calculations. 
If the actual fusion cross sections are lower than the Bass predictions, which is likely [Stic 74], the resulting upper limit of $\tau_{D}$ would become smaller. If the actual fusion cross sections are higher than the Bass predictions, the resulting upper limit of $\tau_{D}$ would be more relaxed. In the low-energy regime $(<60 \mathrm{MeV})$, however, the given upper limits, which remain very small in the case of Os (see Fig. 4.2), hold regardless of the uncertainties in the fusion cross sections $\sigma_{0}$.

\subsection{Simulation of Transient Time Effects on Total Fission Probabilities}

It may be instructive to calculate the total fission probabilities of all chances (up to the point where all of the excitation energy has been exhausted) as a function of both the initial excitation energy $E$ and an assumed transient time $\tau_{D}$, and compare the calculated total fission probabilities with the experimental values. A calculation of this nature should help either to further our insight on possible transient times or to justify some of the previous assumptions.

To perform such a calculation, a scheme to follow the decay process and calculate multichance fission probabilities must be developed. It turns out that such a scheme can be implemented rather simply, as follows:

Consider the decay chain Eq. 4.2. Let $N_{0}(t), N_{1}(t), N_{2}(i), \cdots, N_{i}(t), \cdots$, be the numbers of nuclei $(Z, A),(Z, A-1),(Z, A-2), \cdots,(Z, A-i), \cdots$, respectively, at time $t$ (starting with 1 compound nucleus at $t=0: N_{0}(0)=1, N_{1}(0)=0, N_{2}(0)$ 
$=0, \cdots)$. Assuming a step function for the transient time effect, for an assumed transient time $\tau_{D}$, the number of nuclei $(Z, A-i)$ must satisfy the balance equations:

$$
\begin{aligned}
& \frac{d N_{i}(t)}{d t}=\lambda_{n}^{(i-1)} N_{i-1}(t)-\lambda_{n}^{(i)} N_{i}(t), \quad\left(t \leq \tau_{D}\right) \\
& \frac{d N_{i}(t)}{d t}=\lambda_{n}^{(i-1)} N_{i-1}(t)-\lambda_{C N}^{(i)} N_{i}(t), \quad\left(t \geq \tau_{D}\right)
\end{aligned}
$$

where $\lambda_{n}^{(i-1)}$ and $\lambda_{n}^{(i)}$ are the neutron decay constants of the nuclei $(Z, A-i+1)$ and $(Z, A-i)$, respectively; $\lambda_{C N}^{(i)}$ is the total decay constant of the nucleus $(Z, A-i)$, and $\lambda_{C N}^{(i)}=\lambda_{n}^{(i)}+\lambda_{f}^{(i)}$ with $\lambda_{f}^{(i)}$ being the fission decay constant.

The solution of the above equations is straightforward:

$$
\begin{aligned}
N_{i}(t)=\sum_{j=0}^{j=i} a_{i, j} \exp \left(-\lambda_{n}^{(j)} t\right), \quad j=0,1,2, \cdots, i, \quad\left(t \leq \tau_{D}\right) \\
a_{i, j}=\frac{\lambda_{n}^{(i-1)} a_{i-1, j}}{\lambda_{n}^{(i)}-\lambda_{n}^{(j)}}, \quad j=0,1,2, \cdots, i-1, \\
a_{i, i}=-\sum_{j=0}^{j=i-1} a_{i, j}, \\
a_{0,0}=1.0 ; \\
N_{i}(t)=\sum_{j=0}^{j=i} b_{i, j} \exp \left(-\lambda_{C N}^{(j)} t\right), \quad j=0,1,2, \cdots, i, \quad\left(t \geq \tau_{D}\right) \\
b_{i, j}=\frac{\lambda_{n}^{(i-1)} b_{i-1, j}}{\lambda_{C N}^{(i)}-\lambda_{C N}^{(j)}}, \quad j=0,1,2, \cdots, i-1, \\
b_{i, i}=\exp \left(\lambda_{C N}^{(i)} \tau_{D}\right)\left[N_{i}\left(\tau_{D}\right)-\sum_{j=0}^{j=i-1} b_{i, j} \exp \left(-\lambda_{C N}^{(j)} \tau_{D}\right)\right] \\
b_{0,0}=\exp \left(\left(\lambda_{C N}^{(0)}-\lambda_{n}^{(0)}\right) \tau_{D}\right) .
\end{aligned}
$$

This solution, so written as above, also provides the algorithm to follow the decay chain 4.2 until all the excitation energy is exhausted.

With the solution $N_{i}(t)(\mathrm{i}=0,1,2, \cdots)$ in hand, the total fission probabilities 
$P_{f}^{t}$ can be simply calculated as

$$
P_{f}^{t}=\sum_{i=0} P_{f}^{i \text { th }}=\sum_{i=0} \int_{\tau_{D}}^{\infty} \lambda_{f}^{(i)} N_{i}(t) d t=\sum_{i=0}\left(\frac{\lambda_{f}^{(i)}}{\lambda_{\mathrm{CN}}^{(i)}}\right) \int_{\tau_{D}}^{\infty} N_{i}(t) d\left(\frac{t}{\tau_{\mathrm{CN}}^{(i)}}\right)
$$

where $\tau_{\mathrm{CN}}^{(i)}=1 / \lambda_{\mathrm{CN}}^{(i)}$; the fission decay constants $\lambda_{f}^{(i)}$ and the total decay constants $\lambda_{\mathrm{CN}}^{(i)}$ can be estimated with Eqs. 1.9 and 1.10 .

Now we calculate the total fission probability for the nucleus ${ }^{187} \mathrm{Os}$, given a transient time $\tau_{D}$. The fission barriers $B_{f}^{*}$ and the level density parameters of the nuclei ${ }^{187} \mathrm{Os},{ }^{186} \mathrm{Os},{ }^{185} \mathrm{Os}$ used to estimate the corresponding $\lambda_{f}$ 's, are from the fits to the low-energy portion ( $\leq 75 \mathrm{MeV}$ ) of the corresponding fission excitation functions using the fusion cross sections from the Bass model. Both the fission excitation functions and the corresponding fits are shown in Fig. 3.4. The $B_{f}^{*}$ and $a_{f} / a_{n}$ values extracted from the fits are listed in Table 3.1. $a_{n}$ is taken to be $A / 8\left(\mathrm{MeV}^{-1}\right)$. For isotopes lighter than ${ }^{185} \mathrm{Os}$ in the decay chain Eq. $4.2, a_{f} / a_{n}$ was taken to be the same as that for ${ }^{185} \mathrm{Os}$, and $B_{f}^{*}$ was taken to be the corresponding liquid drop barrier corrected for shell effects. The shell effects of all the nuclei involved are taken to be the nominal values from Möller et al. [Möll 93], some of which are listed in the column $\Delta_{\text {FRDM }}^{n-1}$ of Table 3.1. The results of this calculation for a s€iies of assumed transient times are shown in Fig. 4.4. A comparison of the calculated total fission probability with the corresponding experimental value indicates that the transient time $\tau_{D}$ is not larger than $30 \times 10^{-21}$ sec.

There are two major deficiencies in this calculation. First, the decay scheme does not include decay channels such as $p, \alpha, \cdots$, emissions. This over simplistic 


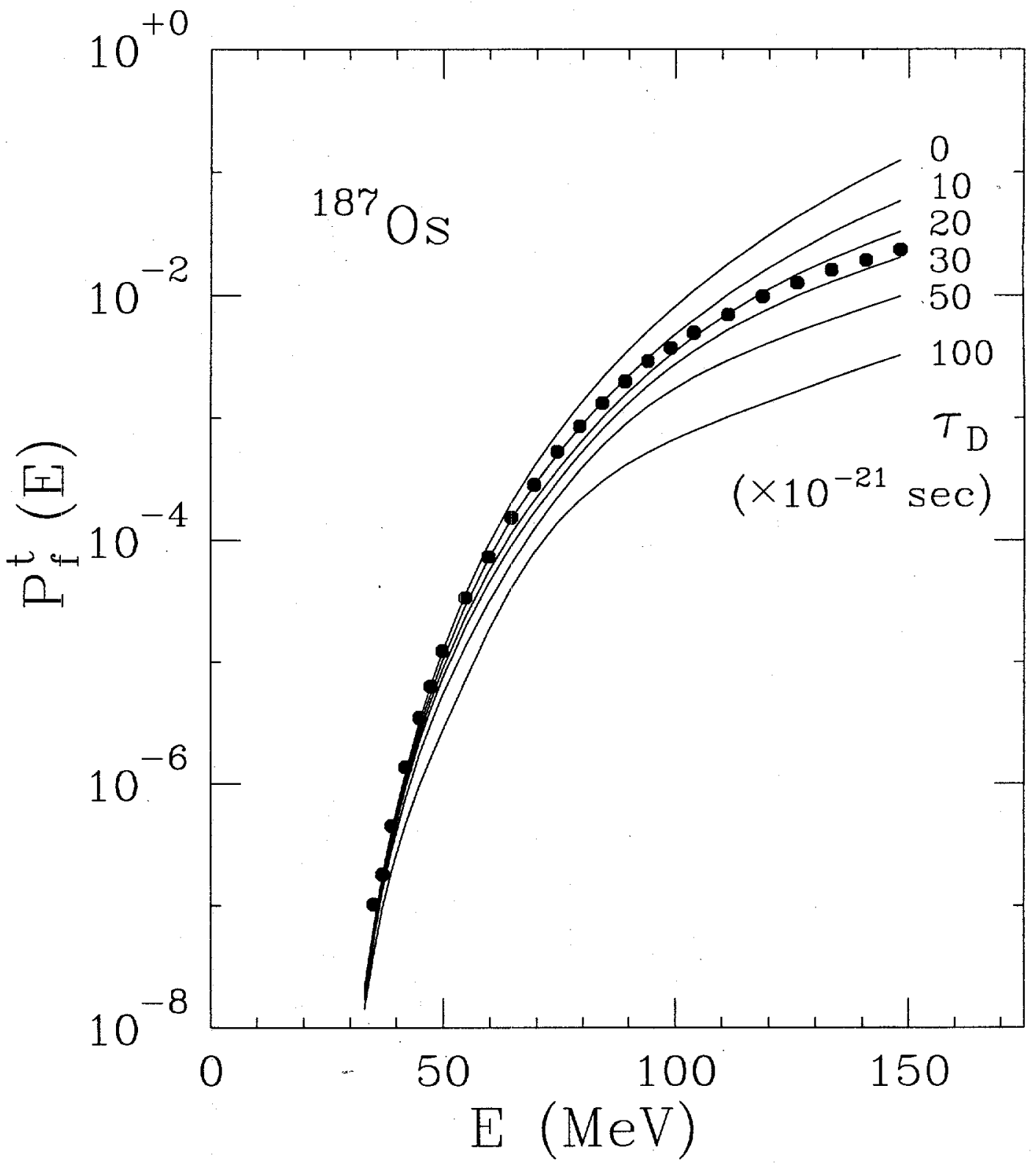

Figure 4.4: Calculated total fission probabilities (solid lines) as a function of excitation energy for a series of assumed transient time $\tau_{D}$ ranging $(0-100) \times 10^{-21} \mathrm{sec}$, for compound nucleus ${ }^{187}$ Os. The experimental total fission probabilities (solid symbols), determined as the ratios of the fission cross sections to the fusion cross sections predicted with the Bass Model, are also shown. A comparison of the calculated total fission probabilities with the corresponding experimental values indicates that $\tau_{\mathcal{D}}$ is smaller than $30 \times 10^{-21} \mathrm{sec}$. See text. 
decay scheme tends to result in a substantial overestimation of the fission probabilities for higher chance fission, and consequently requires a larger transient time to lower the calculated total fission probability to its corresponding experimental value. Second, while the fit to only the low-energy portion of the excitation function results in an accurate fission barrier $B_{f}$, the same fit tends to give too large a value of $a_{f} / a_{n}\left(\sim 1.09\right.$ in the cases of $\left.{ }^{187} \mathrm{Os},{ }^{186} \mathrm{Os},{ }^{185} \mathrm{Os}\right)$. This very large value of $a_{f} / a_{n}$ tends to greatly overestimate the fission probabilities, and consequently requires a significantly larger transient time to lower the calculated total fission probability. Both of the deficiencies reinforce the conclusion that the result from the above simple calculation, i.e., the transient time can not be larger than $30 \times 10^{-21} \mathrm{sec}$, is very conservative.

\subsection{Resetting the Decay Clock at Each Step in the Decay}

\section{Chain}

If the decay clock is assumed to reset at each step in the decay chain, Eq. 4.3 should be rewritten as

$$
P_{f}^{t}(Z, A, E)=P_{f}^{1 s t}+\left(1-P_{f}^{1 s t}\right) P_{f}^{\prime}+\left(1-P_{f}^{1 s t}\right)\left(1-P_{f}^{\prime}\right) P_{f}^{\prime \prime}+\cdots
$$

where

$$
\begin{aligned}
& P_{f}^{\prime}=P_{f}\left(Z, A-1, E-\Delta E_{1}\right) e^{-\tau_{D} / \tau_{n}^{\prime}}, \\
& P_{f}^{\prime \prime}=P_{f}\left(Z, A-2, E-\Delta E_{1}-\Delta E_{2}\right) e^{-\tau_{D} / \tau_{n}^{\prime \prime}}
\end{aligned}
$$


Solving this equation in the same ways as discussed in Section 4.1 gives more stringent upper limits for the transient time, for both Os and Po isotopes. For example, for osmium isotopes with ${ }^{187} \mathrm{Os}$ at the highest excitation energy of $148 \mathrm{MeV}$, taking $P_{f}(Z, A, E)$ and $P_{f}\left(Z, A-1, E-\Delta E_{1}\right)$ to be the measured values $P_{f}^{t}\left({ }^{187} \mathrm{Os}, E\right)$ and $P_{f}^{t}\left({ }^{186} \mathrm{Os}, E-\Delta E_{1}\right)$, repectively, and taking $\left(1-P_{f}^{1 s t}\right)\left(1-P_{f}^{\prime}\right) P_{f}^{\prime \prime}+\cdots$ to be the measured value $P_{f}^{t}\left({ }^{185} \mathrm{Os}, E-\Delta E_{1}-\Delta E_{2}\right)$, solving Eq. 4.11 gives an upper limit of $11 \times 10^{-21} \mathrm{sec}$ for the transient time $\tau_{D}$. In the same way, for polonium produced in ${ }^{3} \mathrm{He}$-induced fission with ${ }^{211} \mathrm{Po}$ at the highest excitation energy of $144 \mathrm{MeV}$, assuming the "resetting" of decay clock leads to an upper limit of $23 \times 10^{-21} \sec$ for $\tau_{D}$.

\subsection{First Chance Fission Probabilities}

Assuming that either the decay clock is reset after each step in the decay chain, or that the fission transient time is zero, Equation 4.3 can be rewritten as

$$
\begin{aligned}
& P_{f}^{t}(A, Z, E)=P_{f}^{1 s t}+\left(1-P_{f}^{1 s t}\right) P_{f}^{t}\left(A-1, Z, E-\Delta E_{1}\right), \\
& P_{f}^{t}\left(A-1, Z, E-\Delta E_{1}\right)=P_{f}^{2 n d}+\left(1-P_{f}^{2 n d}\right) P_{f}^{3 r d}+\cdots,
\end{aligned}
$$

where $P_{f}^{t}\left(A-1, Z, E-\Delta E_{1}\right)$ is the total fission probability of the nucleus $(A-1, Z)$ with excitation energy $E-\Delta E_{1}$. Since both $P_{f}^{t}(A, Z, E)$ and $P_{f}^{t}\left(A-1, Z, E-\Delta E_{1}\right)$ can be determined experimentally, the first chance fission probabilities $P_{f}^{1 s t}$ can thus be obtained, given the above assumptions. The assumption of the zero transient time or resetting of the decay clock, is rather subjective, however, since the justification may be otherwise, as seen in Section 4.1. 
Nevertheless, it is of great interest to determine empirically the first chance fission probabilities, since the first chance fission probabilities are expected to be very sensitive to, and therefore may reveal, transient time effects. Rearranging Eq. 4.12, one obtains:

$$
P_{f}^{1 s t}=\frac{P_{f}^{t}(A, Z, E)-P_{f}^{t}\left(A-1, Z, E-\Delta E_{1}\right)}{1-P_{f}^{t}\left(A-1, Z, E-\Delta E_{1}\right)} .
$$

This is the recipe used by Natowitz et al. [Nato 90] to extract the first chance fission probabilities.

The first chance fission probability obtained with Eq. 4.13 is, unfortunately, sensitive to the errors in both $P_{f}^{t}(A, Z, E)$ and $P_{f}^{t}\left(A-1, Z, E-\Delta E_{1}\right)$, since the errors get amplified by taking the difference of the total fission probabilities of neighboring isotopes. The total fission probabilities $P_{f}^{t}(A, Z, E)$ and $P_{f}^{t}\left(A-1, Z, E-\Delta E_{1}\right)$ can be determined as the ratio of the fission cross section and the formation cross section of the fissioning nucleus. While the fission cross sections presented in this work were measured very precisely, the formation cross sections are much less well known. One has to resort to the theoretical models to calculate the formation cross sections for the fissioning nuclei. As shown in Section 3.1, the formation cross sections calculated. with the Bass model [Bass 74], the Extra-push model [Swia 82], and the Optical model [Macf 78], differ quite substantially. The uncertainties in these formation cross sections result in large errors for the total fission probabilities.

However, an examination of where the errors for the calculated fusion cross sections may lie, should indicate where the resulting first chance fission probabilities may err, and therefore provide guidance on how the first chance probabilities 
extracted with Eq. 4.13 are to be interpreted. In Fig. 3.1, the reaction cross sections calculated with the Optical model [Macf 78], and the fusion cross sections predicted by both the Extra-push model [Swia 82] and the Bass model [Bass74], for the reaction ${ }^{3} \mathrm{He}+{ }^{184} \mathrm{~W} \rightarrow{ }^{187} \mathrm{Os}$, were shown. The large differences in the formation cross sections predicted by different models are an example of how uncertain these formation cross sections can be. Furthermore, there are few experimental data on the fusion cross sections at energies above $70 \mathrm{MeV}$. Notice that the Bass model predicts the lowest fusion cross section among the above mentioned models at energies above $70 \mathrm{MeV}$. There are indications, however, that even the Bass model may overestimate the fusion cross sections at high energy [Stic 74]. The fusion cross sections from the Bass model were chosen in the analysis presented in the previous sections of this chapter. At high energies, the resulting total fission probabilities are most likely underestimated, and underestimated even more as the energy gets higher. The first chance fission probabilities obtained with these underestimated total fission probabilities are, therefore, likely to be the lower limits of the true first chance fission probabilities.

Although it may still be worthwhile to extract the first chance fission probabilities, keeping in mind that the extracted first chance fission probabilities would be the lower limits, due to the large uncertainties in the experimental total fission probabilities, it is better to defer this effort until more accurate formation cross sections of the fissioning nuclei become available. 


\subsection{Summary}

In summary, we have found a new and straight forward way to determine the upper limit of the transient time of fissioning systems, set by the fission probabilities of neighboring isotopes. The upper limit of the transient time is set at $15 \times 10^{-21} \mathrm{sec}$ for Os isotopes, and at $25 \times 10^{-21} \mathrm{sec}$ for Po isotopes. We then conclude that most, if not all, of the transient time as determined from excess amounts of prescission particle emissions, is, therefore, postsaddle. 
Part II

\section{Particle Structure Functions}




\section{Chapter 5}

\section{Introduction}

In Part I, we have studied the nuclear decay process at one extreme - nuclear fission. Through the detailed analysis of the experimental fission excitation functions, we have obtained with good accuracy some of the nuclear properties such as fission barriers, shell effects, etc.. The fission probabilities, even at the highest excitation energies studied, were found to be interpretable based on the transition state formalism, without invoking some of the heavily advertised theoretical effects such as nuclear viscosity. A novel approach has also been developed to determine an upper limit of the fission transient time.

It's only natural to look next at the nuclear decay process at the other extreme - light particle evaporation. An experiment was thus proposed and performed to study the excitation functions of light charged particles emitted from compound nuclei formed in ${ }^{3} \mathrm{He}$-induced reactions. A comparison of the measured excitation functions against the transition state predictions should provide us another check on 
the validity of the transition state formalism. Of particular interest are the ratios of the emission cross sections of the particles of the same charge but of different mass, such as $p / d / t,{ }^{3} \mathrm{He} /{ }^{4} \mathrm{He} /{ }^{6} \mathrm{He}$, etc., since, by taking the ratio, some common factors, such as the formation cross sections which are not well known, can be divided out.

Soon after the experiment was carried out, however, all our attention was drawn to the study of new physics - the existence of complex particles as independent particles inside the nucleus, and their stuctures. The particle spectra obtained from this experiment lent themselves to our search in them for structural modulations similar to those expected from optical potentials. The original physics goal has thus been put aside, and the attention will not return to it as far as this thesis is concerned.

I now turn to this new subject.

\subsection{Particle Structure Functions}

The existence of complex particles in a nucleus may be compared to that of a solute molecule in a solvent. The effect of the solvent on a solute molecule varies from a modest modification of its properties to full dissociation. Particles $(\alpha, d, t$, etc.) inside a nucleus can be seen as solutes in the nuclear solvent. Their existence as independent particles in the nuclear medium relates directly to the question of the extent to which the total nuclear wave function is factorizable into the product of the wave function of the particle and that of the residual nucleus. Qualitatively one can expect that tightly bound particles such as an $\alpha$ particle may have more than a 
fleeting existence in the nucleus, certainly much longer than that of weakly bound particles such as the deuteron.

The interaction of the particles with the nuclear medium can be studied through their evaporation from compound nuclei. Suppose that a complex particle pre-exists in a compound nucleus before it is emitted. As the particle is segregated from a compound nucleus state and prepares to exit, it senses its environment. This environment could be a mean field, like the shell/optical model potential, or a local polarization field. This should result in states which acquire a width through their coupling with the continuum and the remaining many-body degrees of freedom. A strength function should arise which modulates the spectrum of the emitted particles. This is illustrated qualitatively in Fig. 5.1 [More 97b]. The states inside and above the
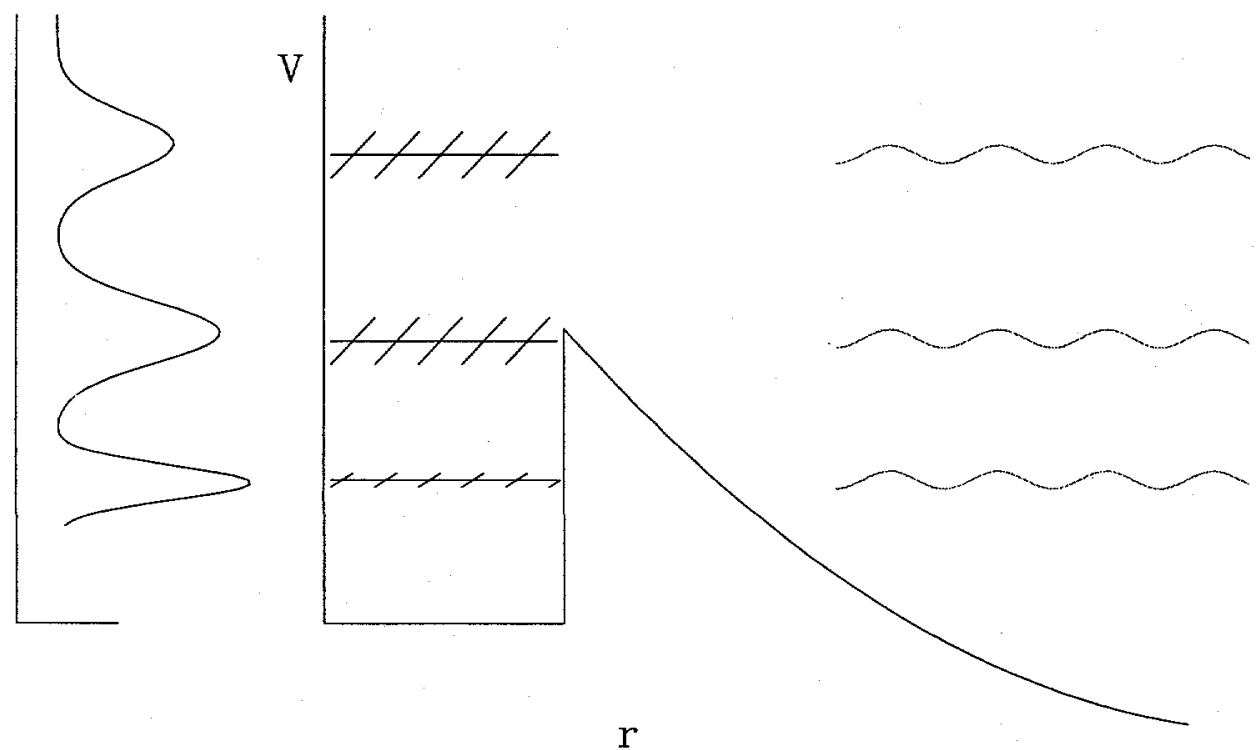

Figure 5.1: Schematic drawing of the states of a particle in potential well [More 97b]. 
well are the states of the particle in the nucleus, which, in the case of a proton, tend to be the shell model states well below the barrier and the optical model resonances in the continuum above the barrier.

An optical resonance is illustrated in Fig. 5.2, where the reaction cross section (top panel) calculated with an optical model [Fesh 54, Macf 78] using an optical potential for ${ }^{4} \mathrm{He}+{ }^{208} \mathrm{~Pb}$ reaction is shown. In this calculation the potential has been modified so that the imaginary part is only $30 \%$ of its normal value in order to enhance the resonance structure. In an effort to isolate this structure the cross section has been fitted with a smooth "background" which has been substracted out (bottom panel).

The optical modulations illustrated in Figs. 5.1 and 5.2 should superimpose on the bulk of the evaporation spectrum determined by phase space, and may become observable in evaporation spectra of very high statistical quality. Since the particle to be emitted is in a hot nucleus whose excitation energy is under experimental control, - the strength function obtained from the modulation of the spectrum refers to that specific excitation energy or temperature. It is thus possible to study the modulation not only for a variety of particles, but also for different temperatures.

In this part, I will present some tentative evidence for the optical modulations in spectra of $\alpha$ particles evaporated from indium compound nuclei produced in the reaction ${ }^{3} \mathrm{He}+{ }^{\text {nat }} \mathrm{Ag}$ at bombarding energies ranging from 55 to $110 \mathrm{MeV} . \mathrm{I}$ will also present the orthogonal polynomial analysis, an approach which has been developed to represent the modulations in the measured $\alpha$-spectra as a combination 


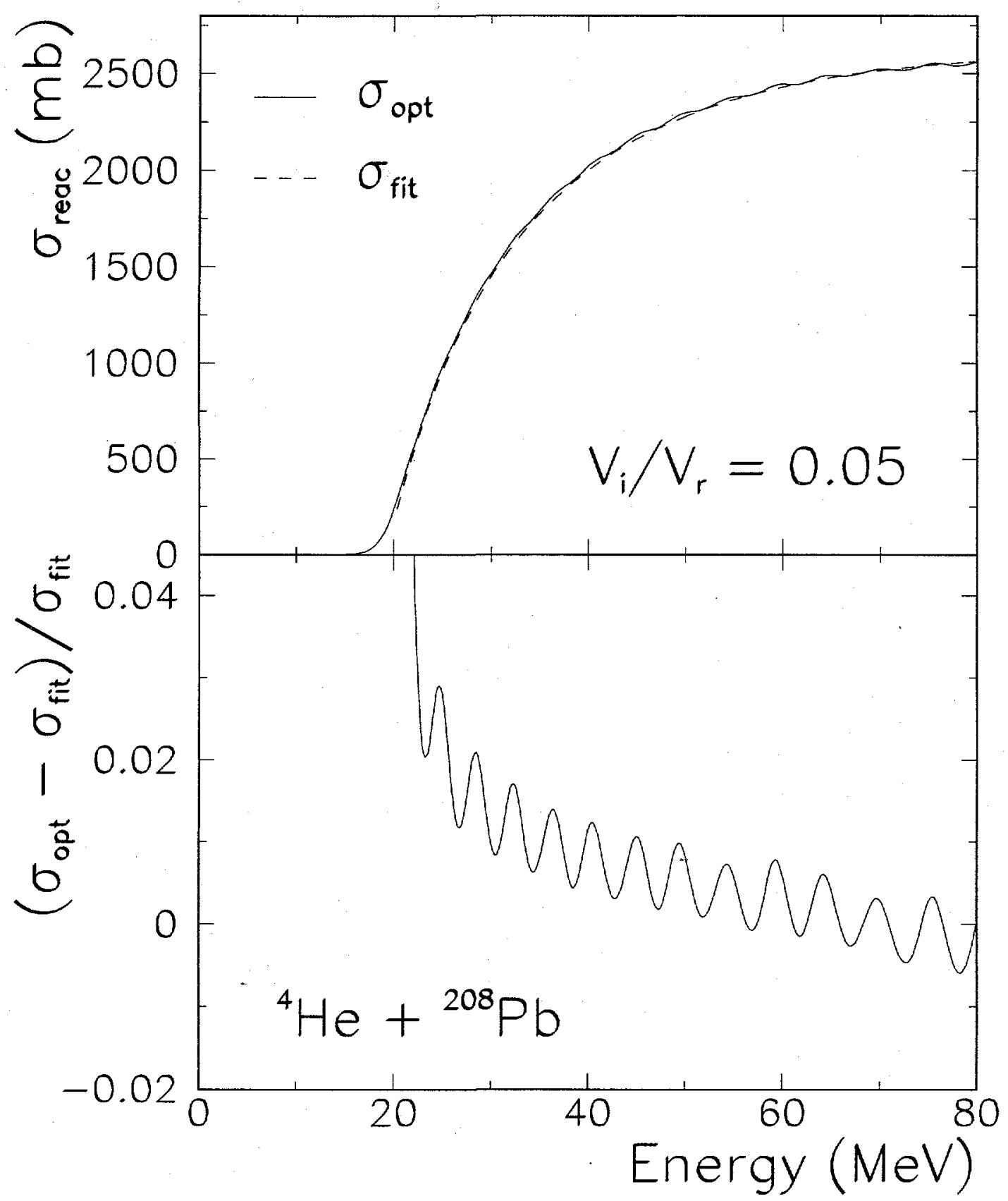

Figure 5.2: Top panel: The reaction cross section as a function of energy calculated for ${ }^{4} \mathrm{He}+{ }^{208} \mathrm{~Pb}$ using an optical model (solid line). In order to enhance the optical resonance, the imaginary part of the optical potential has been reduced in the calculation from $16 \%$ to $5 \%$ of the real potential. The dashed line is a smooth fit to reproduce the general trends of the cross section. Bottom panel: Fractional deviation of the optical model calculation from the smooth fit. 
of orthogonal modes. This approach is potentially applicable to a wide range of problems.

\subsection{Inverse Cross Section}

Another way of looking at the presumed particle structures is to consider the standard expression for the evaporation spectrum. Detailed balance between the compound nucleus states $a$ and the final states $b$ requires that

$$
\rho_{a} \Gamma_{a \rightarrow b}=\rho_{b} \Gamma_{b \rightarrow a}
$$

where $\Gamma_{a \rightarrow b}$ and $\Gamma_{b \rightarrow a}$ are the direct and the inverse decay widths, and $\rho_{a}, \rho_{b}$ are the corresponding phase space volumes. The inverse width is formally expressed in terms of the "inverse" cross section $\left(\sigma_{\text {inv }}\right)$ :

$$
\Gamma_{b \rightarrow a}=\hbar \frac{\sigma_{\mathrm{inv}} v}{\mathrm{~V}}
$$

where $v$ is the velocity of the particle, and $\mathrm{V}$ is a normalization space volume.

The combination of Eq. 5.1 and Eq. 5.2 gives the decay width differential in the particle kinetic energy $(\epsilon)$ :

$$
\Gamma(\epsilon) d \epsilon \propto \sigma_{\mathrm{inv}} \epsilon \rho(E-B-\epsilon) d \epsilon
$$

where $B$ is the particle binding energy. A first order expansion of the log of the level density in the kinetic energy of the particle gives the transparent form:

$$
\Gamma(\epsilon) d \epsilon \propto \sigma_{\text {inv }} \epsilon e^{-\epsilon / T} d \epsilon
$$


Thus the spectrum is the product of a structure factor, namely the "inverse" cross section, and of a phase space factor. Removal of the latter should leave the former with its potentially interesting modulations.

\subsection{Shape Polarization and Evaporation Spectra}

In order to exit from a nucleus, a charged particle must overcome the Coulomb barrier. If the nucleus deforms in the direction of emission, the Coulomb barrier decreases. Let us consider a configuration formed by the emitted particle just in contact with the residual nucleus. Now we deform the residual nucleus always keeping the light particle in contact, and we plot the total energy as a function of deformation. The total energy has a minimum at some finite prolate deformation. This is the location of the saddle point, as shown in Fig. 5.3 [More 75, More 87].

The unbound mode, or reaction coordinate, is the distance between centroids. A particle crossing over the saddle point with zero kinetic energy acquires a kinetic energy at infinity smaller than the Coulomb barrier associated with a spherical configuration. This is not subbarrier emission, in the sense that $t$ is not associated with quantum barrier penetration.

Thermal fluctuations along this deformation coordinate $Z$ lead to large fluctuations in the Coulomb interaction energy, as shown in Fig. 5.3. While the total potential energy $V_{T}$ has a minimum at some prolate deformation, the fragmentfragment Coulomb interaction $V_{\text {Coul }}$ is a monotonically decreasing function of defor- 


\section{Saddle Point and Normal Modes}

i) decay mode:

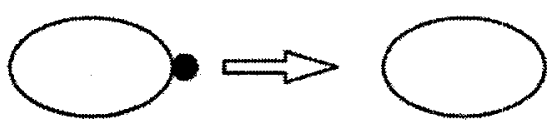

ii) non-amplifying mode:
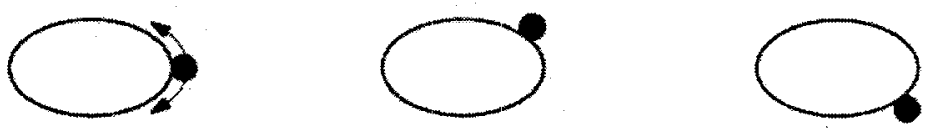

iii) amplifying mode:
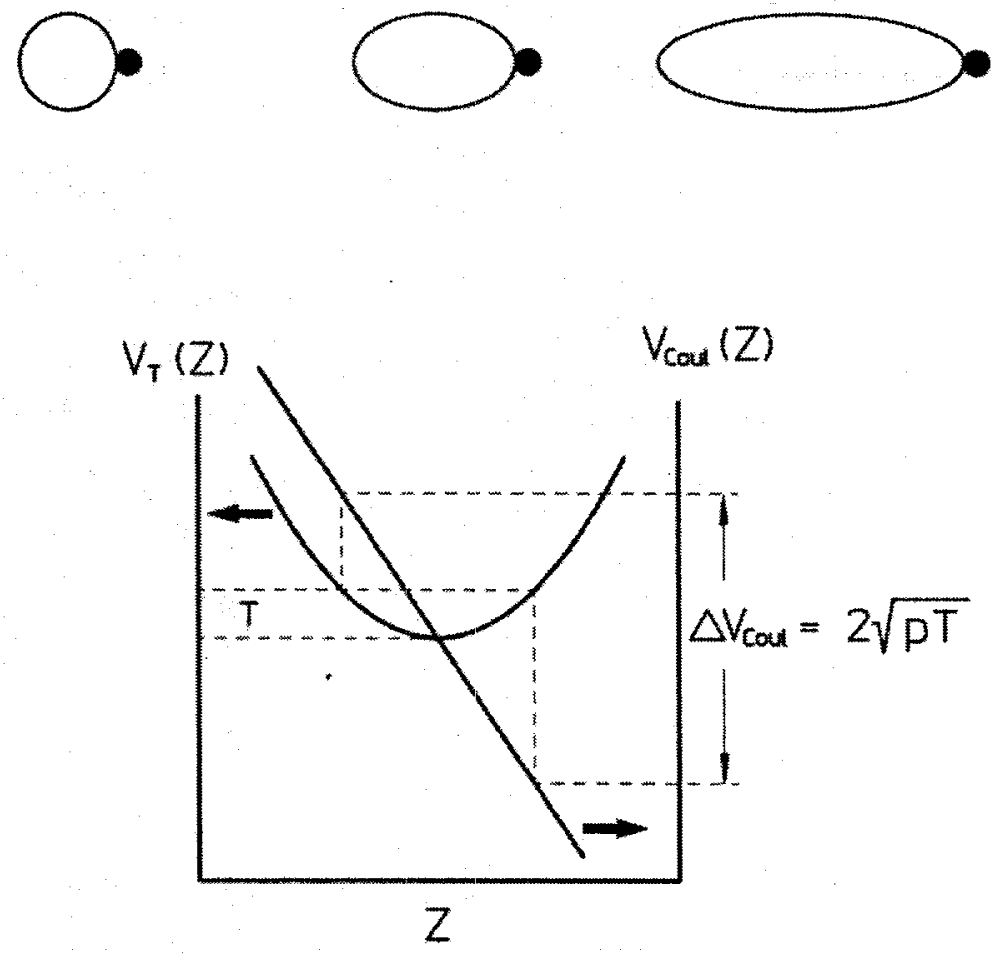

Figure 5.3: Top: Normal modes at the saddle point. Bottom: Total potential energy $V_{\mathrm{T}}$ and Coulomb energy $V_{\text {Coul }}$ as a function of the deformation coordinate $Z$ [More $97 \mathrm{~b}$ ]. 
mation coordinate.

Therefore one can expand the total potential energy and the Coulomb interaction energy about the saddle point along the deformation coordinate $Z(Z=0$ at the saddle):

$$
V_{T}=V_{T}^{0}+k Z^{2}, \quad V_{\text {Coul }}=V_{\text {Coul }}^{0}-c Z
$$

Now, if the shape is allowed to fluctuate involving an energy of the order of the temperature $T$ (see Fig. 5.3), one obtains a corresponding fluctuation of the Coulomb energy:

$$
\Delta V_{\text {Coul }}=2 \sqrt{\frac{c^{2}}{k} T}=2 \sqrt{p T}
$$

The parameter $p\left(=c^{2} / k\right)$ is called the amplification parameter. A degree of freedom with such a general structure is called an amplifying mode.

When the potential energies vary almost exclusively from the Coulomb energy, we have non-amplifying modes. For instance, the oscillation of a particle about - the tip of the prolate core can be considered a non-amplifying modes (see Fig. 5.3). As the particle rolls away from the tip, the Coulomb energy increases because of the decreasing distance between the particle and the residual, while the surface energy of the system changes only in higher order and can be considered approximately constant [More 75].

More quantitative considerations lead to an expression for the kinetic energy spectrum of the particle [More 75]:

$$
P(\epsilon) \propto e^{-x / T} \operatorname{erfc}\left(\frac{p-2 x}{2 \sqrt{p T}}\right)
$$


where $x=\epsilon-V_{\text {Coul }}^{0}$.

We see immediately that, by using a formula like Eq. 5.7 to fit experimental spectra, we can obtain information on the shape polarization associated with particle emission.

Equation 5.7 can be easily generalized to include a number of amplifying and non-amplifying degrees of freedom at the saddle [More 75], and to include the quantum barrier penetration [More 87]. A slightly more sophisticated formula, which incorporates one decay mode, one amplifying mode, and the barrier penetration, has been developed by Moretto and Bowman [More 87]. The formula will be given in Chapter 7 (see Eq. 7.1). It will be shown that this formula can represent alpha evaporation spectra to a remarkable precision. 


\section{Chapter 6}

\section{Measurement of Particle Spectra}

In this chapter, the experiment to measure the energy spectra of the light charged particles $\left(p, d, t,{ }^{3} \mathrm{He}, \alpha\right.$, etc.) emitted from the reactions ${ }^{3} \mathrm{He}+{ }^{197} \mathrm{~A} u$, ${ }^{181} \mathrm{Ta}$, ${ }^{\text {nat }} \mathrm{Ag}$, ${ }^{\text {nat }} \mathrm{Cu},{ }^{27} \mathrm{Al}$ and ${ }^{12} \mathrm{C}$, will be described. The experiment was orginally proposed to study the excitation functions of the light charged particles emitted from the compound nuclei formed in these ${ }^{3} \mathrm{He}$-induced reactions. The particle spectra obtained from this experiment with high statistical quality, however, lent themselves to our search for evidence of the existence of complex particles as independent particles inside a nucleus, and their associated structures.

The experiment was carried out at the 88-Inch Cyclotron of the Lawrence Berkeley National Laboratory. ${ }^{3} \mathrm{He}$ beams of energies 55.0, 65.0, 75.0, 85.0, 95.0, 110.0, 125.0, $140.0 \mathrm{MeV}$ were used to bombard targets made of ${ }^{197} \mathrm{Au},{ }^{181} \mathrm{Ta}$, ${ }^{\text {nat }} \mathrm{Ag}$,

${ }^{\text {nat }} \mathrm{Cu},{ }^{27} \mathrm{Al}$ and ${ }^{12} \mathrm{C}$. The thicknesses of the targets were $1.99,2.10,1.55,1.55,1.56$ and $0.413 \mathrm{mg} / \mathrm{cm}^{2}$, respectively. The uncertainty in the beam energies was $0.3 \%$. 
Two position-sensitive $\Delta E-E$ quad telescopes were used to detect the particles emitted in the reactions. Each quad unit consisted of four separate gas-silicon telescopes, and covered $25^{\circ}$ in plane and $5^{\circ}$ out-of-plane. The active area of each telescope subtended $5^{\circ} \times 5^{\circ}$ and the separation between adjacent telescopes was $1.8^{\circ}$. The gas ionization detectors served as $\Delta E$ detectors, and were operated at a pressure of 51 torr of isobutane gas. The $E$-detector in each telescope unit was a $45 \times 45 \mathrm{~mm}^{2}$ square ( $5 \mathrm{~mm}$ thick) silicon detector with, on the front, strips of low-resistivity material separated by gaps of high-resistivity material to determine and self-calibrate the position [Keho 92, Walt 90]. Using these telescopes, the energy, the atomic number (and the mass number for light charged particles), the in-plane and out-of-plane angles could all be determined for each particle that traversed the $\Delta E$ detector and stopped in the $E$-detector. The out-of-plane angle of the incident particle was determined from the ion drift time in the gas ionization detector. The in-plane angle was determined from a resistive division of the energy sigial from the silicon detector.

Operating at different pressure or with different gas, these same quad units of telescopes have been used previously to detect intermediate mass fragments from boron all the way up to fragments as heavy as the projectiles in the reverse kinematics reactions [Jing 99, Deli 91, Char 90, Han 89].

In this experiment, the detectors were placed at backward angles, with one quad unit on each side of the beam. Relative to the direction of beam, the centers of the eight telescopes were at $116.1^{\circ}, 122.9^{\circ}, 129.8^{\circ}, 136.6^{\circ}, 143.9^{\circ}, 150.7^{\circ}, 157.5^{\circ}$, $164.4^{\circ}$, respectively. 
The particle species were identified from the measured $\Delta E$ and $E$-values. The particle species up to beryllium were resolved both in charge and mass.

The energy calibration of the $E$ and $\Delta E$ detectors was performed using the method illustrated in ref. [McMa 86]. Calibration points were obtained with beams of $20.0 \mathrm{MeV}$ alpha, and $20.6 \mathrm{MeV} \mathrm{H}_{2}^{+1}$ (equivalent of $10.3 \mathrm{MeV}$ proton). These beams were back scattered into the detectors by the Au target. Alpha-particles of energy $6.118 \mathrm{MeV}$ from ${ }^{252} \mathrm{Cf}$ were also used to provide a calibration point at low energy. The energy loss of the calibration particles in the gas section was measured from the difference between the energies deposited in the $E$ detector with and without gas in the ion chamber. Corrections were made for energy losses in the mylar entrance window of the ionization chamber and in a Au absorber used for suppressing electrons and X-rays. Corrections were also made for the energy loss in the Au target. The energy calibrations are accurate to $\pm 1 \%$.

The out-of-plane position was calibrated with a mask, consisting of a matrix of $2 \mathrm{~mm}$ holes separated by $4.73 \mathrm{~mm}$, which could be lowered into position remotely. The in-plane position was self-calibrated [Keho 92]. The typical position resolution obtained was $\pm 0.2^{\circ}$.

The beam charge was collected in a Faraday cup and integrated with a charge-integration module. The integrated charge gives the number of the incident projectiles. Although this information was not used in the current search for the optical modulations in the measured particle spectra, it will allow for the determination of the absolute cross sections of the particles later on. 
For complete fusion reactions, the velocity of the formed compound nucleus $V_{\mathrm{CN}}$ is equal to the center-of-mass (C.M.) velocity. In this case, the velocity and the kinetic energy in the lab frame of the measured particles can be readily transformed into the velocity and the kinetic energy in the source frame (i.e., the center-of-mass frame in complete fusion). The velocity and energy spectra of the particles both in the source frame and in the lab frame can be accumulated at the same time, event-by-event, in the offline analysis.

At high beam energies, incomplete fusion may set in and contribute substantially. The velocities of the nuclei formed in incomplete fusion depend on momentum transfer, and are different from those of the nuclei formed in complete fusion where complete momentum transfer occurs. In light particle $\left({ }^{3} \mathrm{He}\right)$ induced reactions on heavy targets $\left({ }^{\text {nat }} \mathrm{Ag}, \ldots\right.$ ), the velocity of the source from which a particle is emitted is smaller for incomplete fusion than for complete fusion. Thus, in the presence of incomplete fusion, using the center-of-mass velocity to transform the velocity and energy of emitted particles from the lab frame to the source frame may cause distortion of the spectra in the source frame. At $90^{\circ}$, the velocity of a particle in the source frame is the same as the velocity in the lab frame. The tranformation does nothing and therefore no distortion is expected. As a particle is emitted increasingly backwards, the source velocity plays an increasing role in the transformation of the velocity and energy between the two reference frames. At the most backward angle $\left(180^{\circ}\right)$, the velocity of a particle in the source frame is equal to the sum of the particle's velocity in the lab frame and the source velocity. Thus, at angle $180^{\circ}$, the 


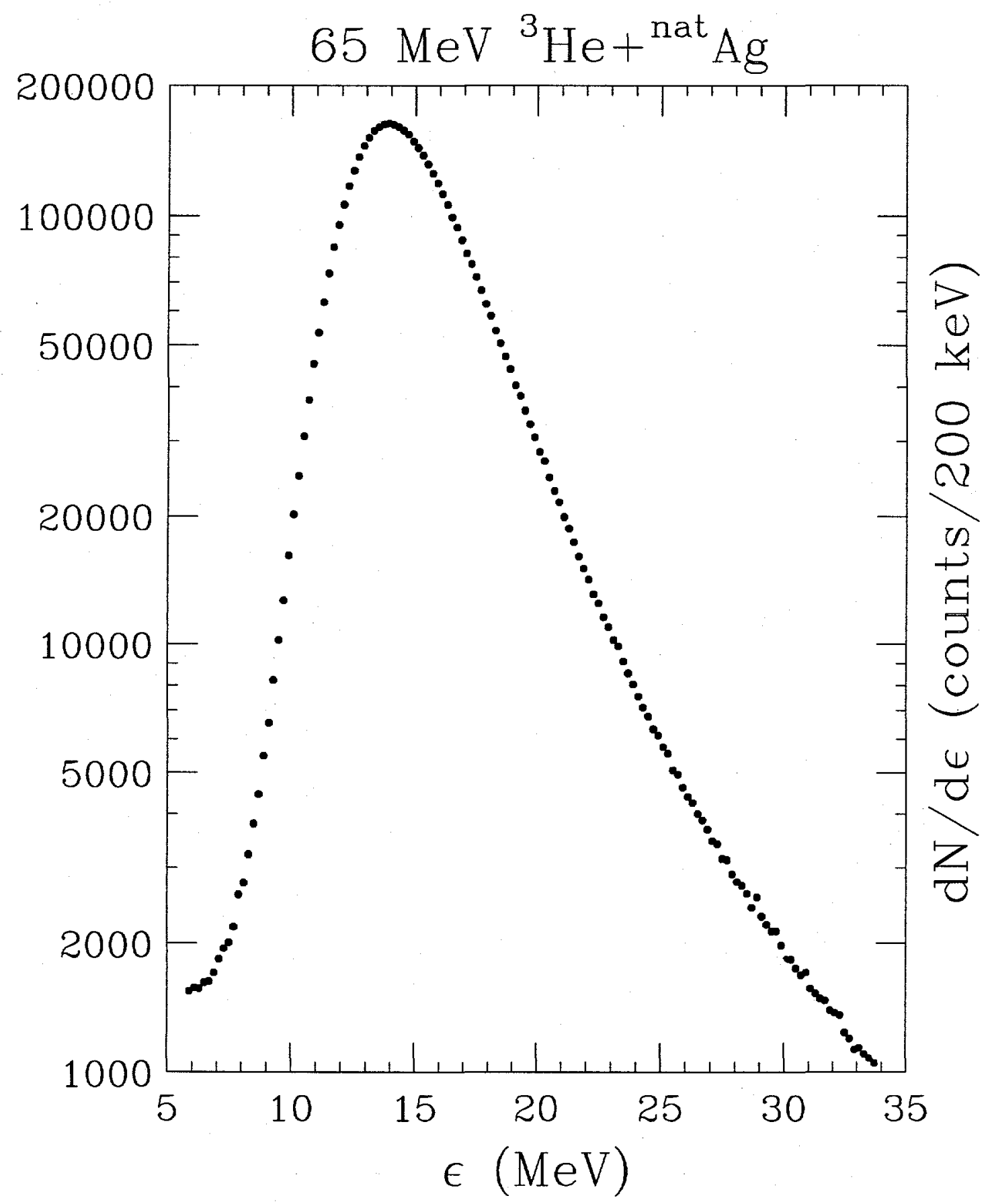

Figure 6.1: The center-of-mass energy spectrum of alpha particles emitted from the ${ }^{3} \mathrm{He}+$ ${ }^{\text {nat }} \mathrm{Ag}$ reaction at $65 \mathrm{MeV}$ beam energy. 
velocity and kinetic energy spectra should appear harder due to the distortion by the transformation from the lab frame to the source frame using the center-of-mass velocity as the source velocity, if incomplete fusion contributes substantially. In other words, in the presence of incomplete fusion, the spectra in the C.M. frame should show a gradual (apparent) hardening from $90^{\circ}$ to $180^{\circ}$. Conversely, an apparent hardening of the spectra with increasing angle in the C.M. frame should indicate the presence of incomplete fusion.

For the ${ }^{3} \mathrm{He}+{ }^{\text {nat }} \mathrm{Ag}$ reaction at energies of $110 \mathrm{MeV}$ or lower, no such apparent hardening of the C.M. spectra was evident as observing angle increased, indicating a dominance of complete fusion. At energies of $125 \mathrm{MeV}$ or higher, however, an apparent hardening of the C.M. spectra with increasing observing angle became discernible, indicating a presence of incomplete fusion at these higher energies.

In this thesis, only the C.M. energy spectra of $\alpha$-particles emitted from nuclei formed in these ${ }^{3} \mathrm{He}$-induced reactions at beam energies below $110 \mathrm{MeV}$ are presented. These spectra were measured with high statistical quality. As an example, the C.M. energy spectrum of $\alpha$-particles emitted from ${ }^{3} \mathrm{He}+{ }^{\text {nat }} \mathrm{Ag}$ reaction at 65 $\mathrm{MeV}$ beam energy is shown in Fig. 6.1. This composite spectrum was generated by summing together the C.M. energy spectra from several individual telescopes. Other spectra will be shown in the next chapter as the analysis proceeds. 


\section{Chapter 7}

\section{Tentative Evidence for Particle}

\section{Structures in Alpha Spectra}

In this chapter, the high-statistics evaporation spectra of alpha particles emitted from the reactions ${ }^{3} \mathrm{He}+{ }^{\text {nat }} \mathrm{Ag}$ and ${ }^{197} \mathrm{Au}$ at bombarding energies of 55 to $110 \mathrm{MeV}$ are analyzed, in order to determine whether physical modulations similar to those expected from optical potentials are present.

The optical modulations can not only arise from a volume potential well, as illustrated in Chapter 5.1, but may also arise from a potential barrier which a particle on its way out encounters near the nuclear surface. Although the transmission coefficient for an inverted real parabolic potential is smooth by Hill-Wheeler formula [Hill 53], the transmission coefficient in general, for a square barrier for example, oscillates with the energy of a penetrating particle.

We will search for evidence of these optical modulations in the measured 
alpha energy spectra. The plan is to fit the alpha spectra with a smooth function, and to search the residuals for modulations. The functional form chosen for the fit should be smooth, and represent adequately the bulk evaporation spectra that arises solely from the available phase space plus the barrier penetration, thus leaving in the residuals the structural modulations intact.

Standard expressions for the evaporation spectra do not provide adequate fits since they do not incorporate thermal shape fluctuations, quantum barrier penetration, etc.. A transition state formalism, which incorporates both the shape polarizations and the barrier penetration, however, is found to fit the measured spectra remarkably well. This formalism, developed by Moretto and Bowman [More 87], is given in the following.

\subsection{A Transition State Formalism for Evaporation Spectra}

Moretto has developed a transition state formalism, which includes shape polarizations (see Eq. 5.7) [More 75], for kinetic energy spectra of evaporated particles. Eণ. 5.7 can be generalized to include a number of amplifying and non-amplifying degrees of freedom (modes) at the saddle. He has also generalized the formalism to include quantum barrier penetration. With inclusion of one decay mode, one amplifying mode, and the barrier penetration, his formula for evaporation spectra $P(\epsilon)$ is written as follows [More 87]:

$$
P(\epsilon) \propto e^{-x / T}\left\{\operatorname{erf}\left(\left(2 V_{\text {Coul }}^{0}+p\right) / 2 \sqrt{p T}\right)-\operatorname{erf}((p-2 x) / 2 \sqrt{p T})\right.
$$




$$
\begin{gathered}
+\frac{1}{2} e^{-(p-2 x)^{2} / 4 p T}\left[e^{(p-2 x-\gamma p T)^{2} / 4 p T}(1+\operatorname{erf}((p-2 x-\gamma p T) / 2 \sqrt{p T}))\right. \\
-e^{(p-2 x+\gamma p T)^{2} / 4 p T}\left(\operatorname{erf}\left(\left(2 V_{\text {Coul }}^{0}+p+\gamma p T\right) / 2 \sqrt{p T}\right)\right. \\
-\operatorname{erf}((p-2 x+\gamma p T) / 2 \sqrt{p T}))]\}
\end{gathered}
$$

where $x=\epsilon-V_{\text {Coul }}^{0}$ and $\epsilon$ is the kinetic energy of evaporated particle; $V_{\text {Coul }}^{0}$ is the Coulomb barrier; $T$ is the temporature of the residual nucleus; $p$ the amplification parameter, and $\gamma$ a parameter representing the barrier penetrability. In developing the above formula, the barrier penetration probability $F_{\mathrm{BP}}$ has been chosen for simplicity as the form:

$$
F_{\mathrm{BP}}(\epsilon)=\frac{1}{2} e^{\gamma x}, \quad(x<0) ; \quad F_{\mathrm{BP}}(\epsilon)=\frac{1}{2}\left(2-e^{-\gamma x}\right), \quad(x \geq 0)
$$

This function and its first derivative are continuous at $x=0$, and has the qualitative features of penetration and reflection expected for a penetrability function.

In the case where the temperature $T$ is low and the Coulomb barrier $V_{\text {Coul }}^{0}$ is large (for $\alpha$ particles, for example), $\operatorname{erf}\left(\left(2 V_{\text {Coul }}^{0}+p\right) / 2 \sqrt{p T}\right)=1$, and erf $\left(\left(2 V_{\text {Coul }}^{0}+\right.\right.$ $p+\gamma p T)(2 \sqrt{p T})=1$. Taking advantage of these facts, Eq. 7.1 can be rewritten as:

$$
\begin{aligned}
P(\epsilon) \propto & e^{-x / T}\{\operatorname{erfc}((p-2 x) / 2 \sqrt{p T}) \\
& +\frac{1}{2} e^{-(p-2 x)^{2} / 4 p T}\left[e^{(p-2 x-\gamma p T)^{2} / 4 p T} \operatorname{erfc}(-(p-2 x-\gamma p T) / 2 \sqrt{p T})\right. \\
& \left.\left.-e^{(p-2 x+\gamma p T)^{2} / 4 p T} \operatorname{erfc}((p-2 x+\gamma p T) / 2 \sqrt{p T})\right]\right\}
\end{aligned}
$$

Notice that Equations 7.1 and 7.3 do not contain any polynomial of $2^{\text {nd }}$ order or higher in $\epsilon$, and the (complementary) error functions and the exponentials are all smooth functions. 
The complementary error function erfc in Eq. 7.3 can be readily calculated with fractional error everywhere less than $1.2 \times 10^{-7}$ [Pres 92]:

$$
\begin{aligned}
\ln (\operatorname{erfc}(|x|))= & \ln (t)-x^{2}-1.26551223+t \times(1.00002368 \\
& +t \times(0.37409196+t \times(0.09678418+t \times(-0.18628806 \\
& +t \times(0.27886807+t \times(-1.13520398+t \times(1.48851587 \\
& +t \times(-0.82215223+t \times 0.17087277))))))))
\end{aligned}
$$

where $t=\frac{1.0}{1.0+0.5|x|} ;$ and

$$
\operatorname{erfc}(x)= \begin{cases}\exp (\ln (\operatorname{erfc}(x))), & x \geq 0 \\ 2.0-\exp (\ln (\operatorname{erfc}(-x))), & x<0\end{cases}
$$

Equation 7.3 is found to be able to fit the measured alpha spectra to a remarkable precision. This is shown in the following section.

\subsection{Smooth Fit of Alpha Spectra, and Modulation in Residuals}

Now we fit the energy spectra of alpha particles evaporated from nuclei formed in ${ }^{3} \mathrm{He}+{ }^{\text {nat }} \mathrm{Ag}$ reactions with Equation 7.3. The fit will be performed for the spectra in the energy range from 10 to $24 \mathrm{MeV}$. The high-energy tail above $24 \mathrm{MeV}$ is excluded in the fitting to avoid the involvement of pre-equilibrium particle emission, which is not considered in Eq. 7.3. The cut-off point at $24 \mathrm{MeV}$ was determined empirically. The spectra below $10 \mathrm{MeV}$ is also cut off due to possible contributions 
from light contaminants on the surface of the target. The shape of $\alpha$-particle energy spectra for possible light contaminants such as carbon and oxygen should look similar to those for ${ }^{3} \mathrm{He}+{ }^{\text {nat }} \mathrm{Ag}$, but are narrower and peaked at lower energies. The relative contributions from possible light contaminants may be estimated by the relative counts at energies below the evaporation peak $\left(\sim 6 \mathrm{MeV}\right.$ for ${ }^{3} \mathrm{He}+{ }^{\text {nat }} \mathrm{Ag}$ reactions, see Fig. 6.1) of the measured energy spectra.

Shown in the lower panel of Fig. 7.1 are the measured alpha-particle spectrum and the fit for ${ }^{3} \mathrm{He}+{ }^{\text {nat }} \mathrm{Ag}$ reaction at the beam energy of $65 \mathrm{MeV}$. The quality of the fit is remarkable. The exceedingly good quality of the fit indicates that, on the one hand, (the bulk of) the evaporation spectrum is indeed statistical, and that, on the other hand, the shape fluctuations at the saddle point are very well accounted for by Eq. 7.3. The high quality of the fit should also allow for an accurate determination of the amplification parameter $p$, and the Coulomb barrier $V_{\text {Coul }}^{0}$ for $\alpha$-particle evaporation.

The percentage difference between the experimental data and the fit is shown in the upper panel of Fig. 7.1. The residual of the fit is of the order of $1 \%$ throughout the energy range, which shows the goodness of the fitting function. The residual shows a statistically significant modulation with an amplitude of about $1.5 \%$.

The alpha-particle spectra and the corresponding fits with Eq. 7.3 for ${ }^{3} \mathrm{He}$ $+{ }^{\text {nat }} \mathrm{Ag}$ reactions at other bombarding energies ranging from 55 to $110 \mathrm{MeV}$ are shown in the lower panel of each sextant in Fig. 7.2. The percentage residuals of the fits are also shown in Fig. 7.2, in the upper panel of each sextant. All the fits are 


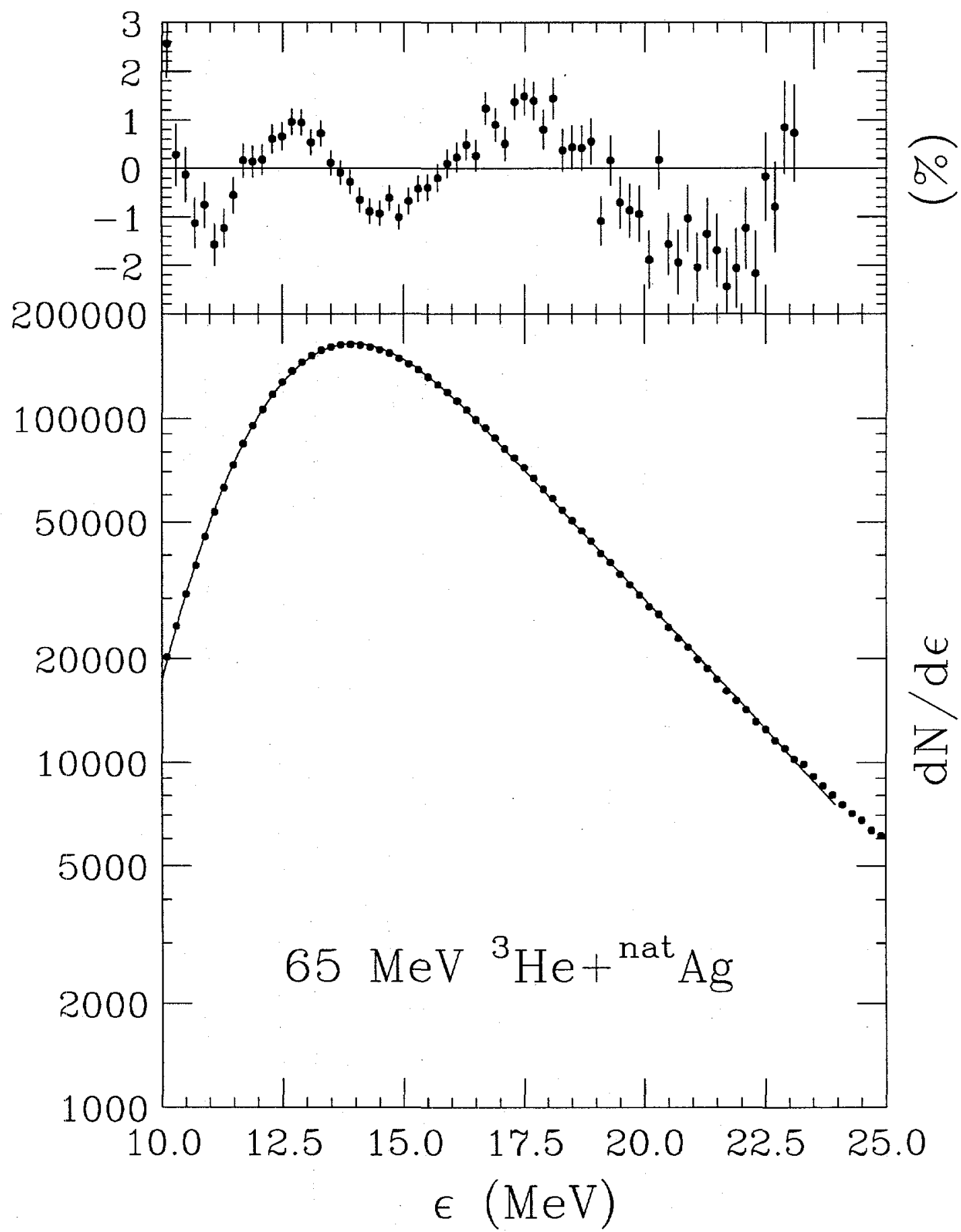

Figure 7.1: Lower panel: The experimentally measured C.M. $\alpha$ energy spectrum $(\bullet)$ from ${ }^{3} \mathrm{He}+{ }^{\text {nat }} \mathrm{Ag}$ reaction at $65 \mathrm{MeV}$ beam energy, and the fit $(-)$ with Eq. 7.3. Upper panel: The percentage difference between the experimental data and the fit shown in the lower panel. The error bars represent the statistical errors of the experimental data. 
excellent, with residuals on the order of only $1 \%$. All the residuals show a statistically significant modulation with an amplitude of about $1.5 \%$ which repeats itself both in amplitude and phase at all bombarding energies.

Several questions immediately come to mind. Is the modulation physical, as those expected for optical potentials? Could it be the results of instrumental effects, like modulations introduced by departures from linearity of ADCs, amplification electronics, detectors, etc.? Or could it be a fitting problem associated with the rigidity or modulations in the fitting function?

Instrumental effects have been ruled out. The modulation is observed in several independent detector-electronics chains. The modulation and the alpha-spectra themselves shown in Fig. 7.2 have also been confirmed in follow-up experiments using different detectors, ADCs, different gains, and different chains of amplification electronics.

The question regarding the fitting function is a more difficult one. The fitting function Eq. 7.3 is non polynomial, thus it does not introduce the modulation we see. From a close inspection it is clear that it is the data that wrap themselves around the fitting function, not vice-versa.

But what if there is a slight mismatch between a smooth fitting function and a true statistical evaporation spectrum which is also smooth? There seems no easy and clear-cut answer to this question.

Before we can answer this question, let us look now at the spectra of alphaparticles emitted from nuclei formed in another ${ }^{3} \mathrm{He}$-induced reaction, ${ }^{3} \mathrm{He}+{ }^{197} \mathrm{Au}$. 


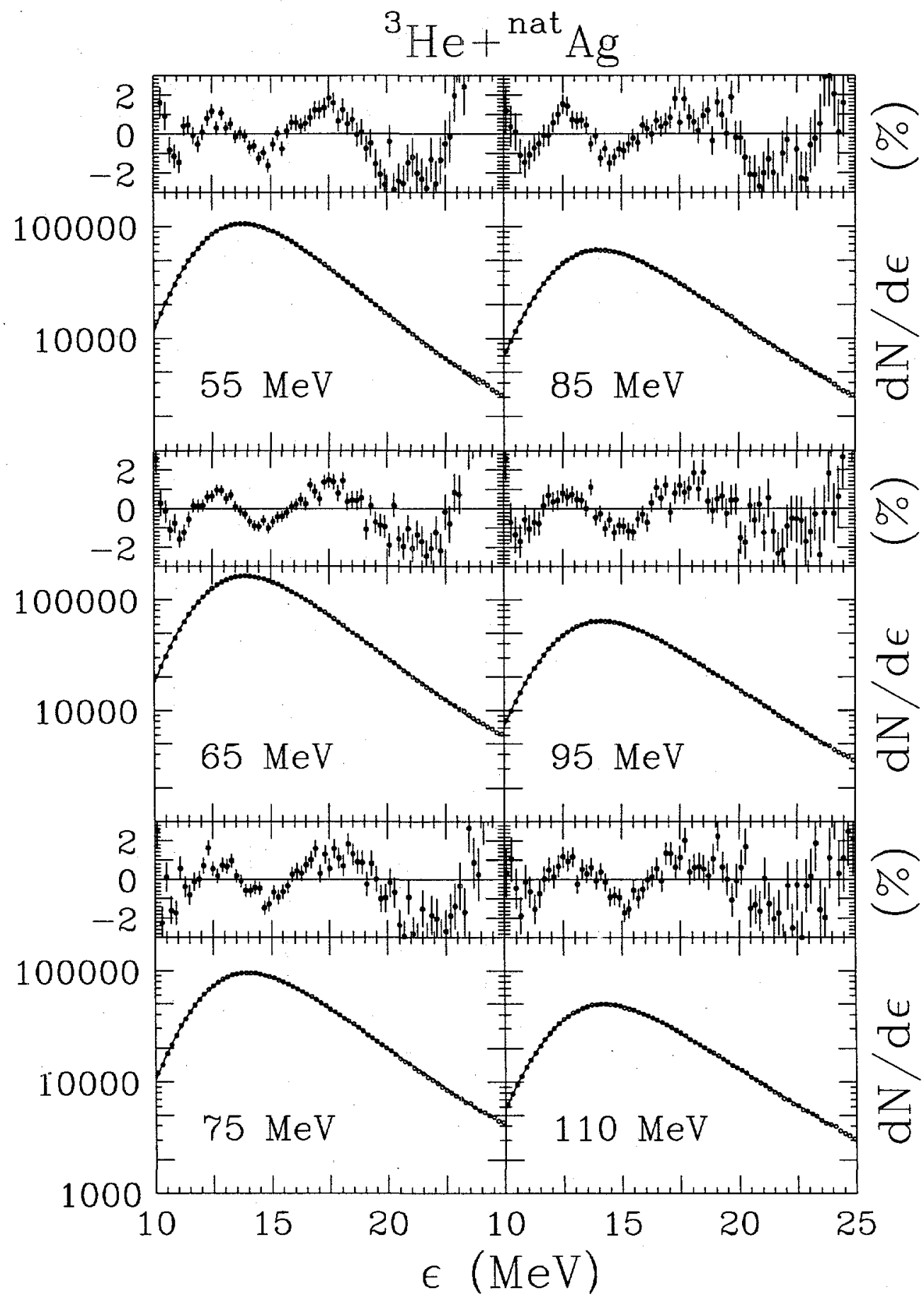

Figure 7.2: Lower panels: The experimentally measured C.M. $\alpha$ energy spectra $(\circ)$ from the ${ }^{3} \mathrm{He}+{ }^{\text {nat }} \mathrm{Ag}$ reactions at $55,65,75,85,95,110 \mathrm{MeV}$ beam energies, and the corresponding fits (-) with Eq. 7.3. Upper panels: The percentage differences between the experimental data and the fits with Eq. 7.3 shown in the lower panels. The error bars represent the statistical errors of the experimental data. 


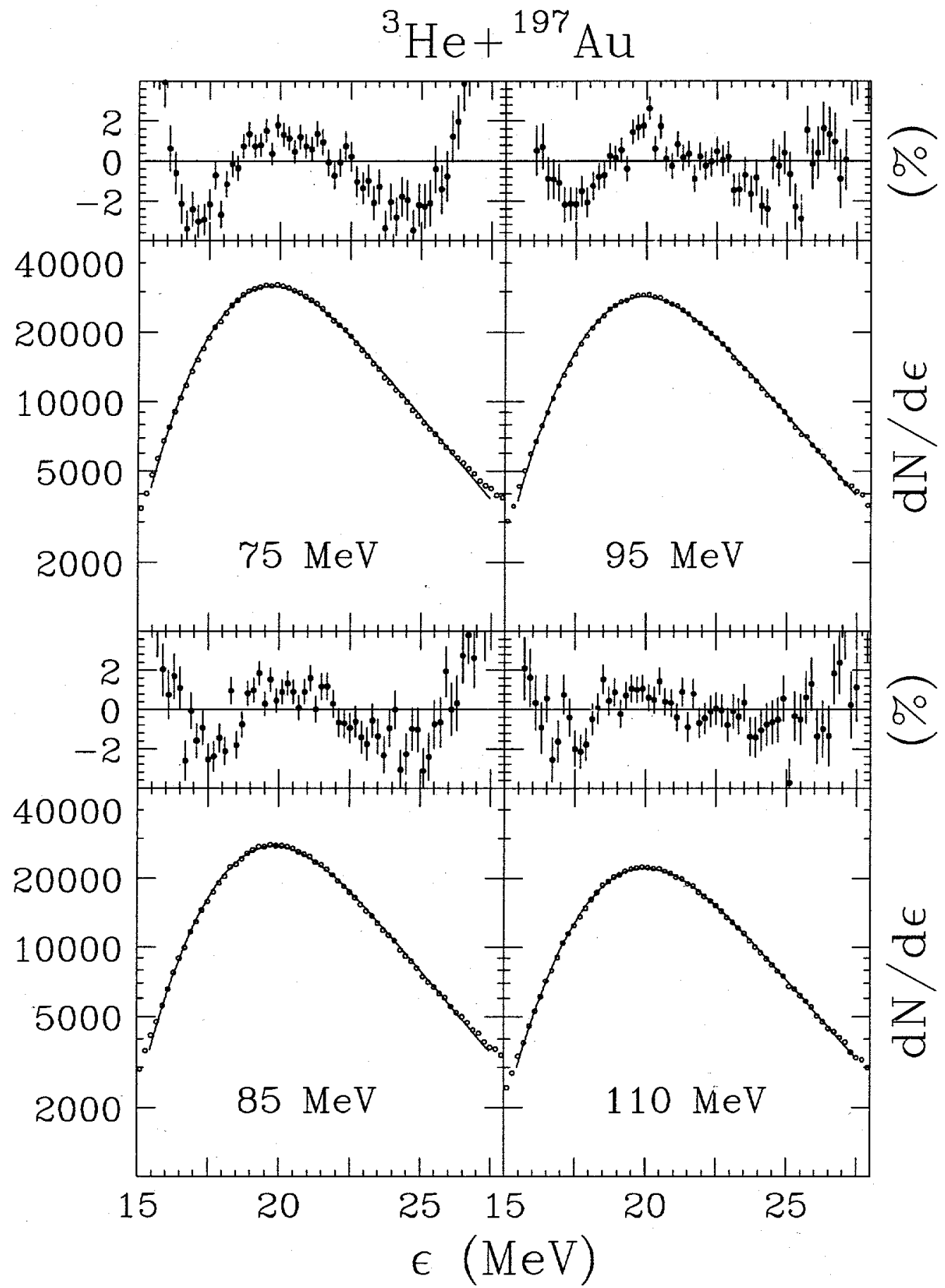

Figure 7.3: Lower panels: The experimentally measured C.M. $\alpha$ energy spectra $(0)$ from the ${ }^{3} \mathrm{He}+{ }^{197} \mathrm{Au}$ reactions at $75,85,95,110 \mathrm{MeV}$ beam energies, and the corresponding fits (-) with Eq. 7.3. Upper panels: The percentage differences between the experimental data and the fits with Eq. 7.3 shown in the lower panels. The error bars represent the statistical errors of the experimental data. 
Shown in the lower panels of sextants in Fig. 7.3 are the alpha-spectra and the corresponding fits with Eq. 7.3 for ${ }^{3} \mathrm{He}+{ }^{197} \mathrm{Au}$ reactions at bombarding energies of $75,85,95,110 \mathrm{MeV}$, respectively. As in the case of ${ }^{3} \mathrm{He}+{ }^{\text {nat }} \mathrm{Ag}$ reaction, the low energy part of the spectra was excluded in the fits because of possible contributions from light surface contaminants, and the high-energy tail of the measured spectra was also excluded because Eq. 7.3 does not consider pre-equilibrium emission. Due to the substantially higher Coulomb barrier for $\alpha$-particle emission, the $\alpha$-particle emission probability is much smaller in the ${ }^{3} \mathrm{He}+{ }^{197} \mathrm{Au}$ reaction than in the ${ }^{3} \mathrm{He}$ $+{ }^{\text {nat }} \mathrm{Ag}$ reaction. The low emission probability, on the one hand, leaves way for pre-equilibrium emission to play a larger role in the high-energy tail of the measured spectra, and on the other hand, elevates the relative importance of the contributions to the low-energy part of the spectra by light surface contaminants. Thus the data range used in the fits was even more limited than in the case of ${ }^{3} \mathrm{He}+{ }^{\text {nat }} \mathrm{Ag}$ reaction.

The quality of the fits is excellent. The residuals of the fits are of the order of $1 \%$. The percentage residuals of the fits are shown in Fig. 7.3 , in the upper panels of sextants. The residuals also show a modulation somewhat similar to that observed in Fig. 7.2 for ${ }^{3} \mathrm{He}+{ }^{\text {nat }} \mathrm{Ag}$ reaction. But, the periodicity of the modulation seen in the spectra from the ${ }^{3} \mathrm{He}+{ }^{\text {nat }} \mathrm{Ag}$ reaction, seems lost, and the modulation seems to decrease in magnitude as energy increases, perhaps due to the more limited data range.

The alpha-spectra from the ${ }^{3} \mathrm{He}+{ }^{181} \mathrm{Ta}$ and ${ }^{\text {nat }} \mathrm{Cu}$ reactions are not shown. The results for ${ }^{3} \mathrm{He}+{ }^{181} \mathrm{Ta}$ reaction look similar to those for the ${ }^{3} \mathrm{He}+{ }^{197} \mathrm{Au}$ 
reaction. Both $\mathrm{Ta}$ and $\mathrm{Cu}$ targets are more susceptible to surface contamination from oxidation. Thus the low-energy part of the alpha-spectra obtained for the reactions with these targets has contributions from light surface contaminants such as carbon and oxygen. For ${ }^{3} \mathrm{He}+{ }^{\text {nat }} \mathrm{Cu}$ reaction, the center-of-mass velocities are high except at the lowest bombarding energies. The presence of incomplete fusion at high bombarding energies therefore makes the C.M. energy spectra different from the energy spectra in the frame of emitting sources. However, the alpha-spectra from the ${ }^{3} \mathrm{He}+{ }^{\text {nat }} \mathrm{Cu}$ reaction at the lowest bombarding energies $(55,65 \& 75 \mathrm{MeV})$ should allow us to extract with good accuracy the Coulomb barrier $V_{\text {Coul }}^{0}$ and the amplification parameter $p$ for the Ga nucleus formed in the reaction. The $\alpha$-particle energy spectra from the ${ }^{3} \mathrm{He}+{ }^{181} \mathrm{Ta}$ reaction at intermediate bombarding energies $(75,85,95 \& 110 \mathrm{MeV})$, where the alpha-emission probability is relatively high, thus diminishing the contributions from light surface contaminants, should also allow us to extract the Coulomb barrier $V_{\text {Coul }}^{0}$ and the amplification parameter $p$ for the Re nucleus. The extracted values for these parameters are presented in the following section.

\subsubsection{Extracted parameters, and Systematics of Coulomb Barriers for} Alpha Evaporation

The high quality of the fits shown in Fig. 7.1, 7.2 and 7.3 indicates that the formalism represented by Eq. 7.3 can well account for the underlying physical process of alpha evaporation. That is: a) particle evaporation is governed by phase space; $b$ ) 
the thermal shape fluctuations at the saddle lead to a distribution of configurations - thus a distribution of Coulomb barriers - at which a particle is emitted. The latter can be well described by the Coulomb barrier $V_{\text {Coul }}^{0}$ at the saddle and the amplification parameter p. The high quality of the fits using Eq. 7.3 thus allows for an accurate determination of $V_{\text {Coul }}^{0}, p$, and the temperature $T$ of the residual nucleus produced by particle emission.

The values for $V_{\text {Coul }}^{0}, p$, and $T$ extracted from the fits shown in Fig. 7.2 for nuclei formed in the ${ }^{3} \mathrm{He}+{ }^{\text {nat }} \mathrm{Ag}$ reactions, are shown in Fig. 7.4, as a function of bombarding energies. In the energies range from $55^{\circ}$ to $110 \mathrm{MeV}$, the values for $V_{\text {Coul }}^{0}$ are constant, indicating virtually the same saddle shapes in this energy range. The amplification parameter $p$ also remains roughly constant. The temperature $T$ of the residual nucleus increases slowly as energy increases, as expected.

The values for $V_{\text {Coul }}^{0}, p$, and $T$ obtained from the fits shown in Fig. 7.3 for nuclei formed in the ${ }^{3} \mathrm{He}+{ }^{197} \mathrm{Au}$ reactions, are shown in Fig. 7.5. The extracted values for these parameters for nuclei formed in the ${ }^{3} \mathrm{He}+{ }^{181} \mathrm{Ta}$ and ${ }^{\text {nat }} \mathrm{Cu}$ are shown in Figs. 7.6 and 7.7, repectively. In the explored energy range, the Coulomb barriers $V_{\text {Coul }}^{0}$ for these systems do not change with energy, as observed in Fig. 7.4 for ${ }^{3} \mathrm{He}+{ }^{\text {nat }} \mathrm{Ag}$. One also observes approximately constant values for the amplification parameter $p$ over the energy range explored. The values for the temperature $T$ increase slowly with energy, but decrease, also slowly, with increasing size of the nucleus.

The Coulomb barriers $V_{\text {Coul }}^{0}$ at the saddle for alpha evaporation, obtained 


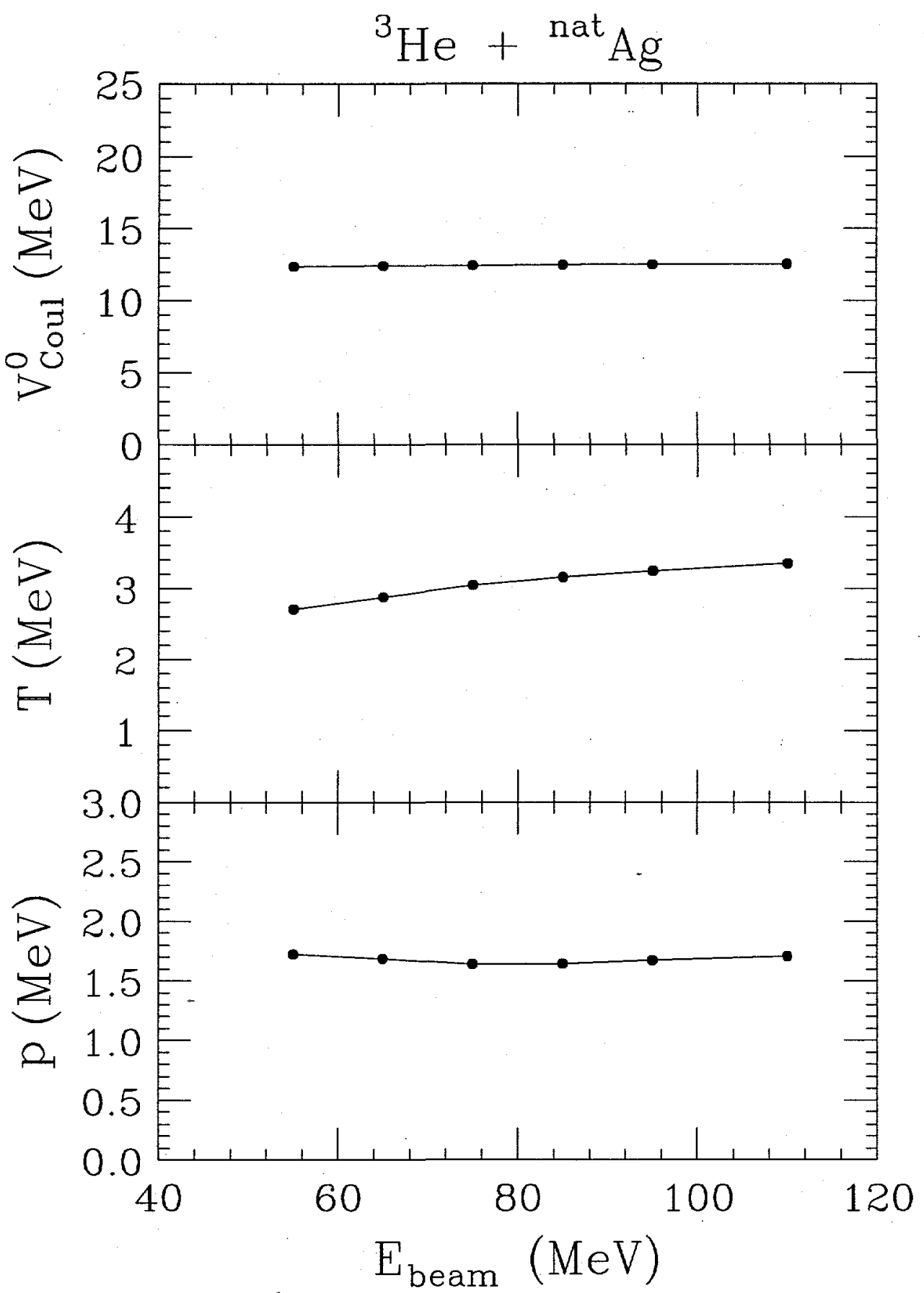

Figure 7.4: The values of the Coulomb barrier $V_{\text {Coul }}^{0}$, the temperature $T$ of the residual nucleus and the amplification parameter $p$, extracted from the fits shown in the lower panels of sextants in Fig. 7.2 for nuclei formed in the ${ }^{3} \mathrm{He}+{ }^{\text {nat }} \mathrm{Ag}$ reactions, are plotted against bombarding energy. The error bars are smaller than the size of symbols. 


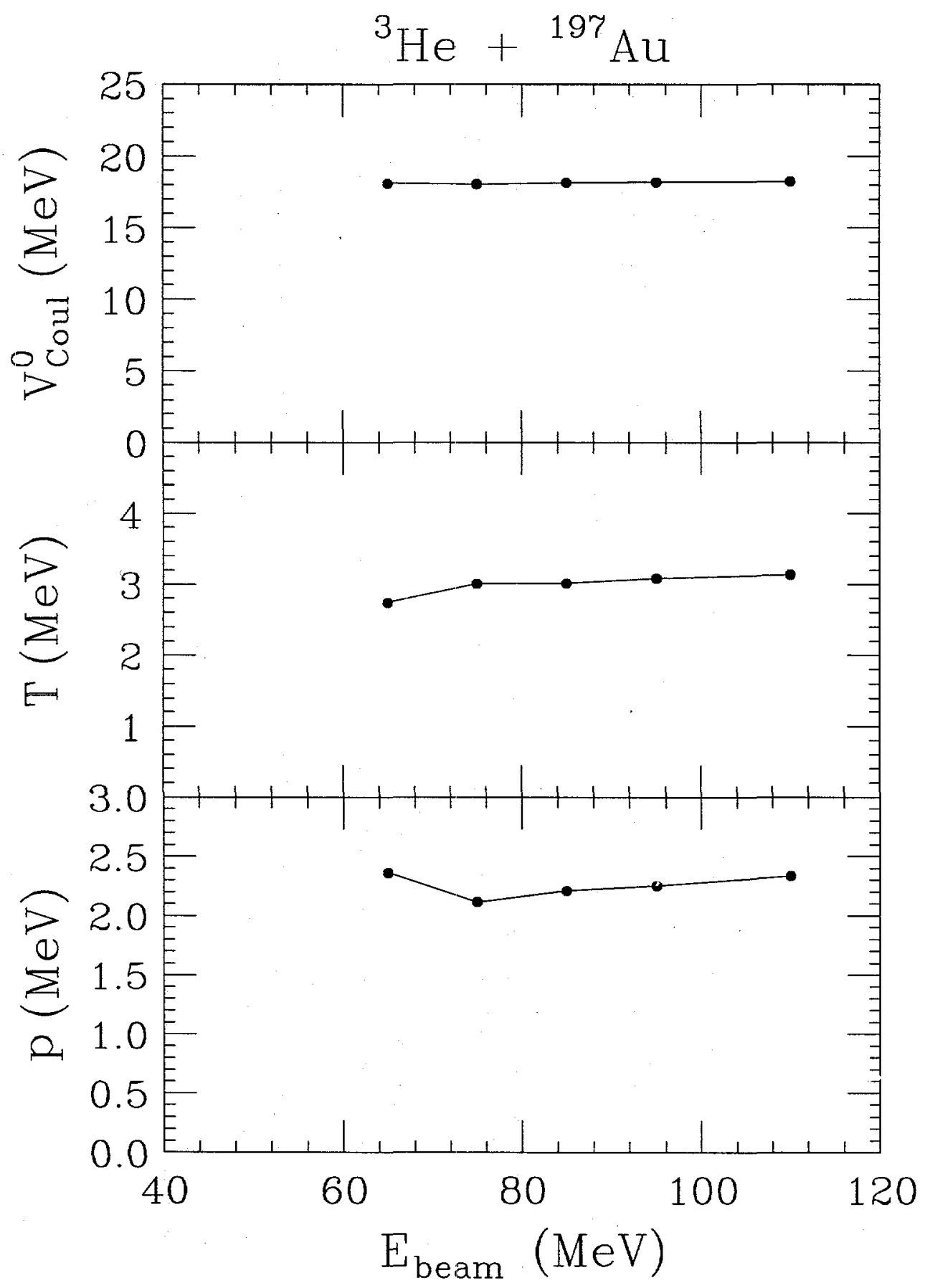

Figure 7.5: The values of the Coulomb barrier $V_{\text {Coul }}^{0}$, the temperature $T$ of the residual nucleus and the amplification parameter $p$, extracted from the fits shown in the lower panels of quadrants in Fig. 7.3 for nuclei formed in the ${ }^{3} \mathrm{He}+{ }^{197} \mathrm{Au}$ reactions, are plotted against bombarding energy. The error bars are smaller than the size of symbols. 


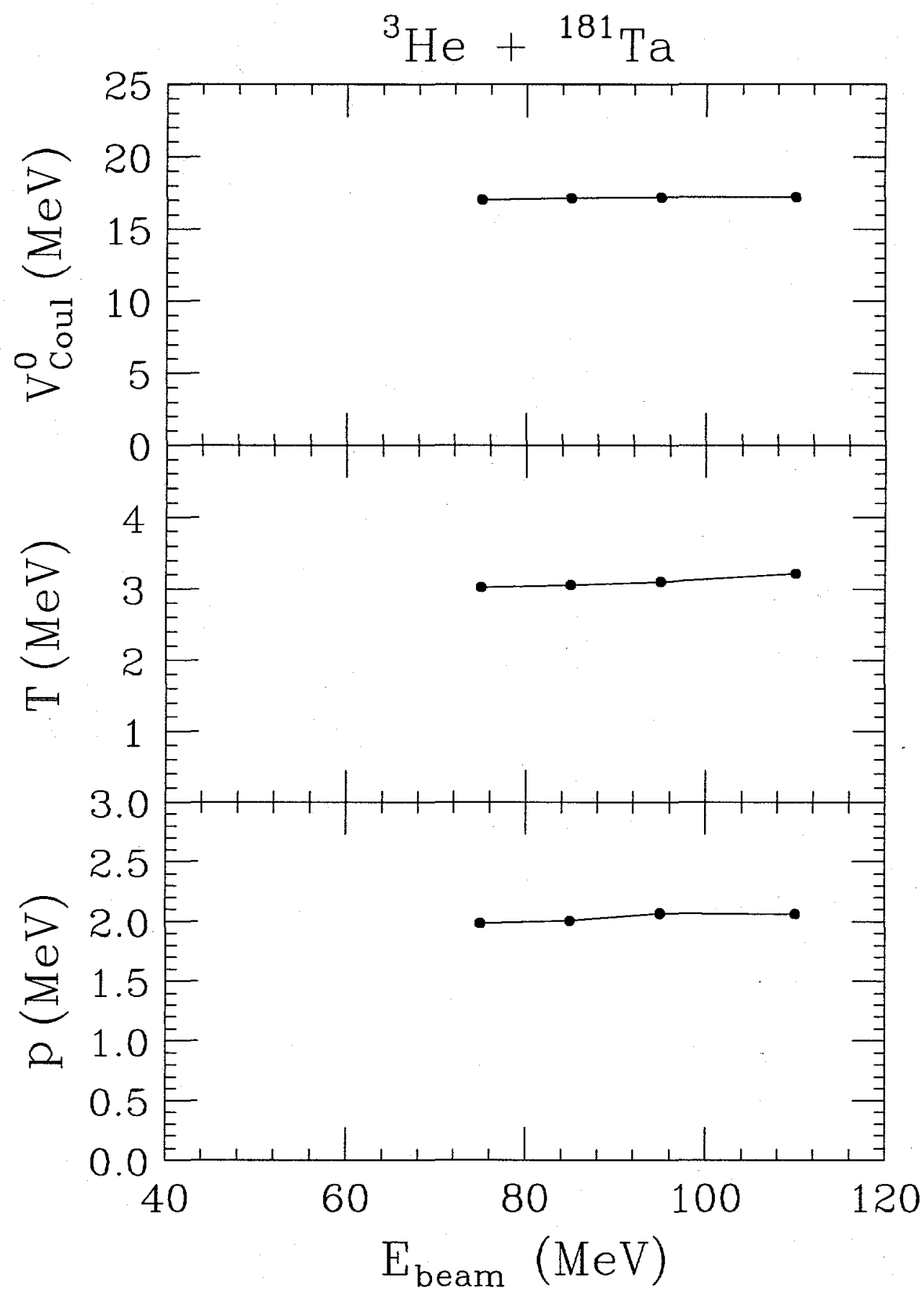

Figure 7.6: The values of the Coulomb barrier $V_{\text {Coul }}^{0}$, the temperature $T$ of the residual nucleus and the amplification parameter $p$, extracted from the fits using Eq. 7.3 to the measured $\alpha$-particle spectra for nuclei formed in the ${ }^{3} \mathrm{He}+{ }^{181} \mathrm{Ta}$ reactions, are plotted against bombarding energy. The error bars are smaller than the size of symbols. 


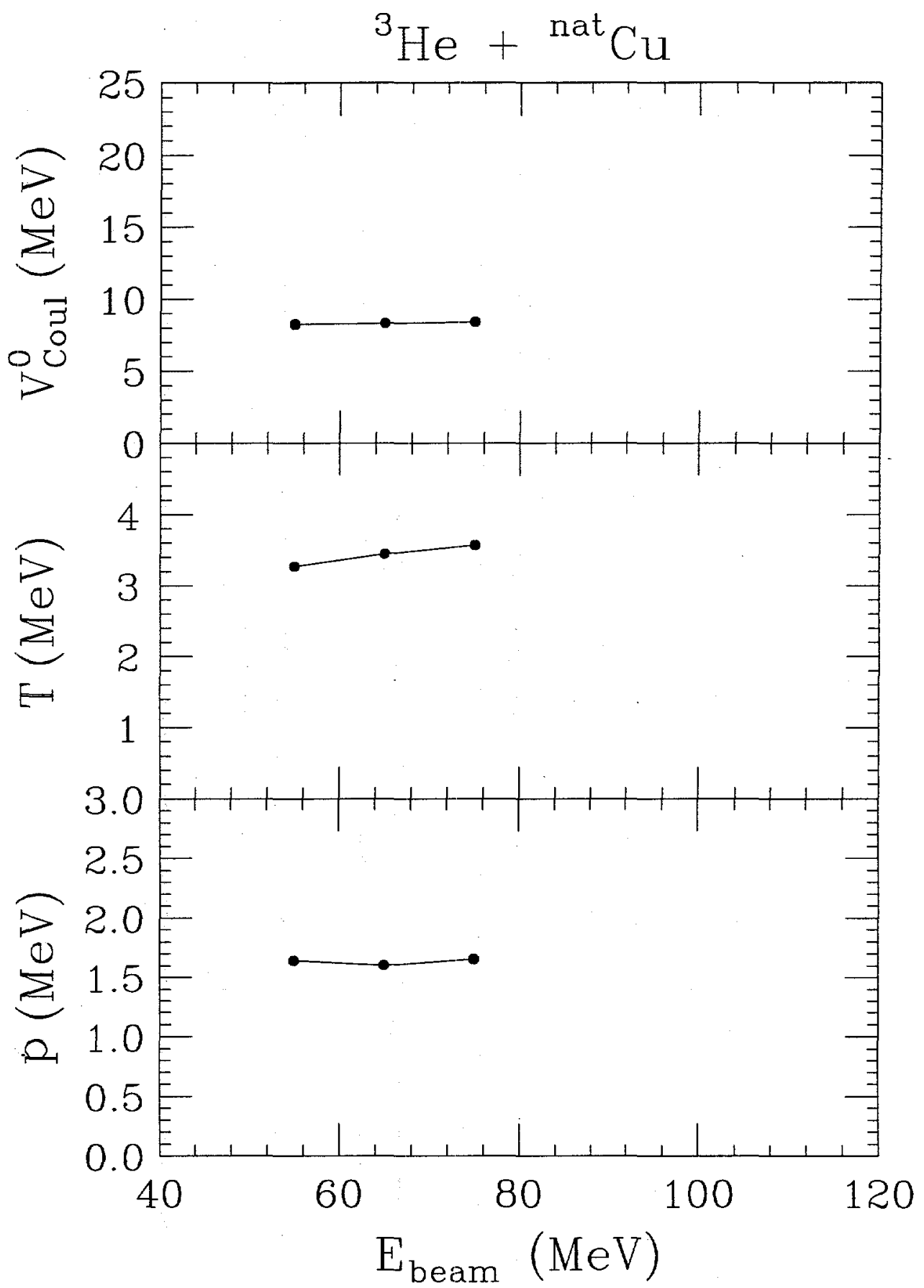

Figure 7.7: The values of the Coulomb barrier $V_{\text {Coul }}^{0}$, the temperature $T$ of the residual nucleus and the amplification parameter p, extracted from the fits using Eq. 7.3 to the measured $\alpha$-particle spectra for nuclei formed in the ${ }^{3} \mathrm{He}+{ }^{\text {nat }} \mathrm{Cu}$ reactions, are plotted against bombarding energy. The error bars are smaller than the size of symbols. 


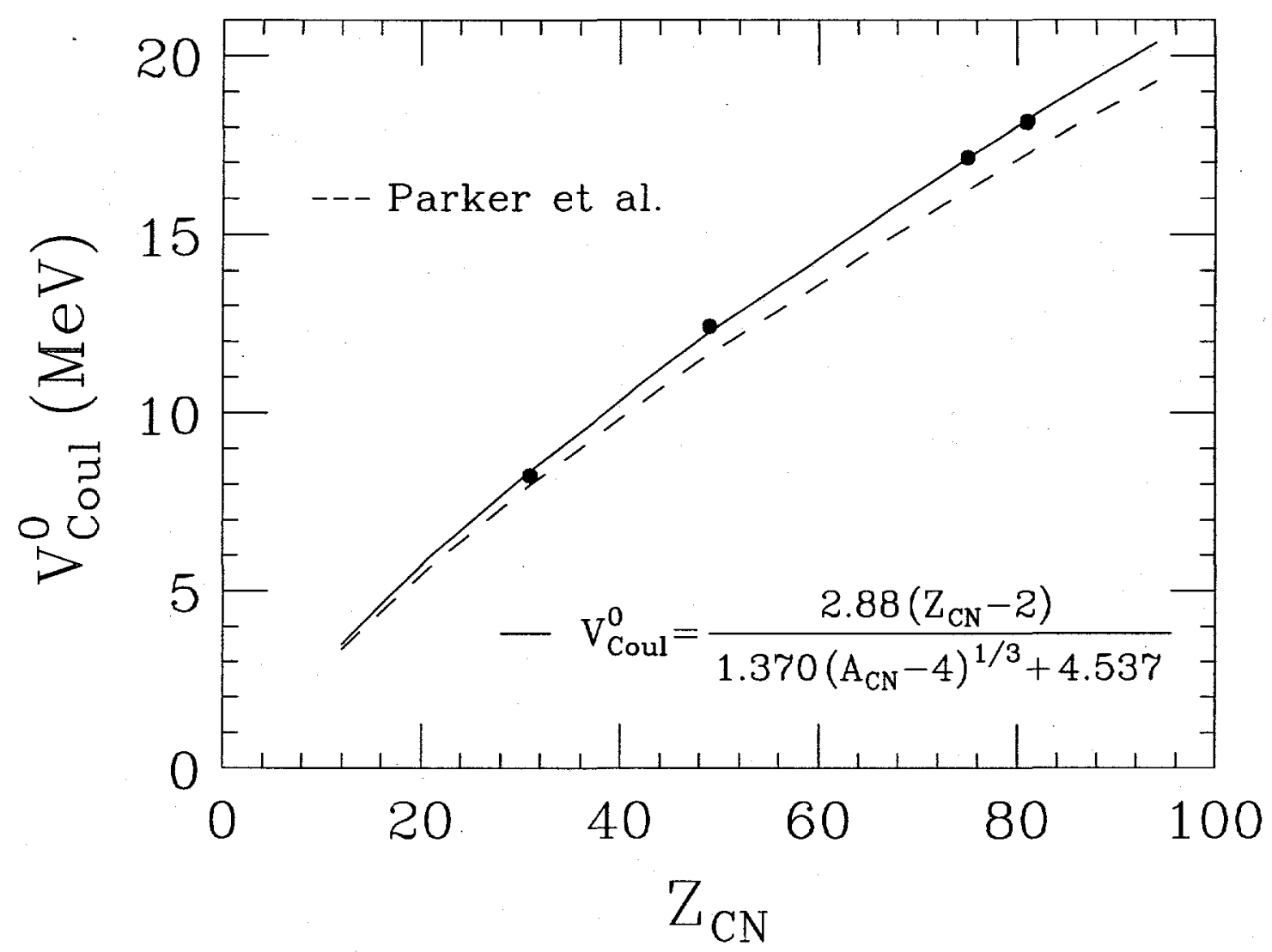

Figure 7.8: The extracted values of the Coulomb barriers $V_{\text {Coul }}^{0}$ for $\alpha$-particle evaporation are plotted versus the atomic number $Z_{\mathrm{CN}}$ of the nucleus from which $\alpha$-particle is emitted. The solid line is the systematic of $V_{\text {Coul }}^{0}$ established with the data points shown. The dashed line is the systematic of the Coulomb barriers for alpha evaporation given by Parker et al. [Park 91].

for a range of nuclei ( $\mathrm{Ga}, \mathrm{In}, \mathrm{Re}, \mathrm{Tl})$ produced in the ${ }^{3} \mathrm{He}+\mathrm{Cu}, \mathrm{Ag}, \mathrm{Ta}, \mathrm{Au}$ reactions, provide a systematic of $V_{\text {Coul }}^{0}$ as a function of the atomic number and the geometrical size of nucleus. This systematic is shown as the solid line in Fig. 7.8 where the Coulomb barrier $V_{\text {Coul }}^{o}$ for alpha evaporation is plotted against the atomic number $Z_{\mathrm{CN}}$ of the compound nucleus from which the alpha particle is emitted. The systematic is well represented by the equation:

$$
V_{\mathrm{Coul}}^{\mathrm{o}}=\frac{2.88\left(Z_{\mathrm{CN}}-2\right)}{1.370\left(A_{\mathrm{CN}}-4\right)^{1 / 3}+4.537}(\mathrm{MeV})
$$


where $A_{\mathrm{CN}}$ is the mass number of the compound nucleus. Also shown in Fig. 7.8 as the dashed line is the systematic of Parker et al. [Park 91] for Coulomb barriers for alpha-particle evaporation. The evaporation barriers from the Parker's systematic are slightly lower than ours. The compound nuclei, whose alpha evaporation barriers were determined and used to establish the Parker's systematic, were prepared in heavy-ion reactions, and have therefore average angular momenta substantially larger than for the compound nuclei produced in the ${ }^{3} \mathrm{He}$-induced reactions. Large angular momenta may result in deformed compound nuclei and thus reduced evaporation barriers. The difference between the Parker's systematic and ours may also lie in the fact that, while our Coulomb barrier is for the saddle configuration, Parker's is averaged over the thermal shape fluctuations.

It should be interesting to see how the amplification parameter $p$ changes with the size of compound nucleus. In Fig. 7.9, the values for $p$ are plotted against the atomic number of the nucleus from which the $\alpha$-particle is emitted. One observes a gradual increase of $p$ as an emitting nucleus becomes larger. This seems natural, since the larger the nucleus, the more Coulomb energy associated with it. No effort has been made yet, however, to understand the detailed systematic features of $p$.

The extracted values for the penetrability parameter $\gamma$ are $20.3,23.9,15.0$, $14.2,16.9,14.9$ for the ${ }^{3} \mathrm{He}+{ }^{\text {nat }} \mathrm{Ag}$ reactions at $55,65,75,85,95,110 \mathrm{MeV}$ bombarding energies, respectively. For ${ }^{3} \mathrm{He}+{ }^{197} \mathrm{Au}$ reactions at the beam energies 75 , $85,95,110 \mathrm{MeV}$, the extracted values for $\gamma$ are $35.1,14.4,15.4,14.4$, respectively. The $\gamma$ values obtained from the fits for alpha emission from the ${ }^{3} \mathrm{He}+{ }^{\text {nat }} \mathrm{Cu} \&{ }^{181} \mathrm{Ta}$ 


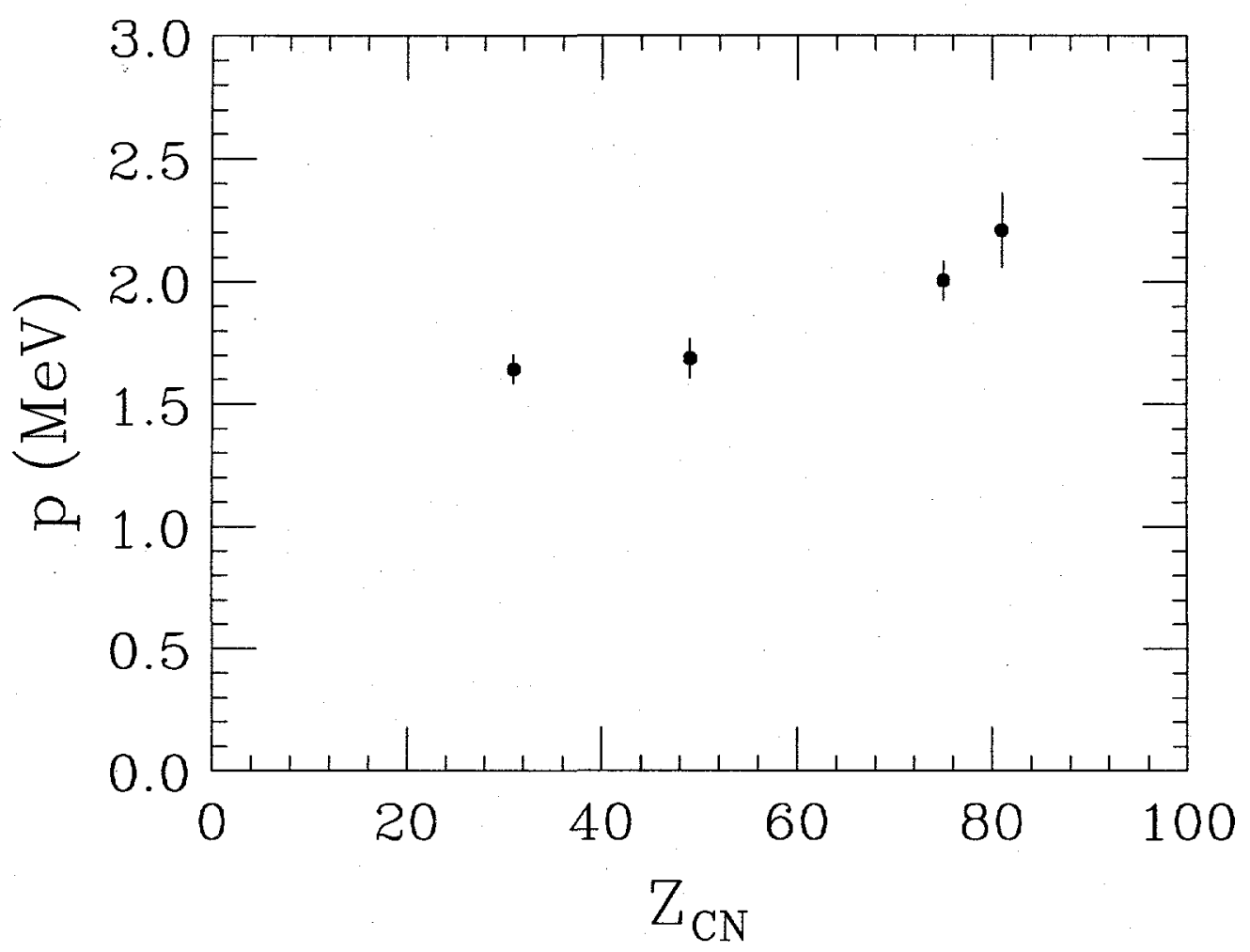

Figure 7.9: The extracted values of the amplification parameter $p$ are plotted versus the atomic number $Z_{\mathrm{CN}}$ of the nucleus from which $\alpha$-particle is emitted.

reactions are also high, all above 12.6. These large values for $\gamma$ indicate that contributions from the quantum barrier penetration are negligible at these excitation energies [More 87].

\subsection{Orthogonal Polynomials Representation of Modulations in Alpha Spectra}

As shown in Section 7.2, the fits with Eq. 7.3 indeed represent very well the spectral shape of the $\alpha$-energy spectra from reactions ${ }^{3} \mathrm{He}+{ }^{\text {nat }} \mathrm{Ag}$ and other reactions at the various excitation energies. Moreover, Eq. 7.3 is a smooth function. 
The residuals of the fits show statistically significant modulations which, in the case of ${ }^{3} \mathrm{He}+{ }^{\text {nat }} \mathrm{Ag}$ reaction in particular, repeat themselves both in amplitude and phase at all bombarding energies. The goodness of the fits with Eq. 7.3 is, however, a posteriori knowledge. We do not know a priori that Eq. 7.3 should work so well.

Therefore, one still needs to seek further assurance that the modulations observed in the residuals are indeed physical resonances. This assurance turns out to be quite difficult to obtain.

To a lesser extent, one can try to quantitatively describe and extract information on the modulations observed in the alpha spectra, perhaps in the hope that some clue may become evident. For this purpose, we have devised an analytical procedure based upon orthogonal polynomials. We write down the experimental spectrum $F(\epsilon)$ as a linear combination of orthogonal polynomials $P_{n}(\epsilon)$

$$
F(\epsilon)=\sum c_{n} S(\epsilon) P_{n}(\epsilon)
$$

where $S(\epsilon)$ is a suitably chosen weight function that generates the polynomials $P_{n}(\epsilon)$; $c_{n}$ is the coefficient which can be considered as the amplitude of a spectral mode corresponding to the $n^{\text {th }}$ order polynomial $P_{n}(\epsilon)$. The orthogonality condition is

$$
\int_{a}^{b} S^{2}(\epsilon) P_{n}(\epsilon) P_{m}(\epsilon) d \epsilon= \begin{cases}0, & n \neq m \\ 1, & n=m\end{cases}
$$

The choice of $S(\epsilon)$ is dictated by the desire to concentrate the bulk of the spectral shape into the single coefficient $c_{0}$. The modulations then appear in the higher-order coefficients, hopefully in only one or two. This goal can be achieved 
by choosing for $S(\epsilon)$ the form given by Eq. 7.3 with parameters obtained from the least squares fit. This guarantees that $c_{0}$ will take up the bulk of the spectrum. The amplitudes $c_{n}$ can be obtained from the dot product of the experimental spectrum with the $n^{\text {th }}$ polynomial

$$
c_{n}=\int_{a}^{b} F(\epsilon) S(\epsilon) P_{n}(\epsilon) d \epsilon
$$

and the corresponding strength $s_{n}$ can be defined as

$$
s_{n}=c_{n}^{2} / \int_{a}^{b} F^{2}(\epsilon) d \epsilon
$$

By definition we have $\sum s_{n}=1$.

For the given weight function $S(\epsilon)$, the existence of the polynomials $P_{n}(\epsilon)$ that satisfy the orthogonality condition Eq. 7.8 can be proved. The proof, and the procedure that is used to generate the polynomials $P_{n}(\epsilon)$, can be found in textbooks, ref. [Sans 59] for example.

$\mathrm{A} \mathrm{C}++$ computer code has thus been developed to implement this analytical procedure. The details of the implementation and the code are given in Appendix B. Now we can apply the procedure to the experimental spectra shown in Section 7.2. The results of this analysis for the alpha spectrum for the ${ }^{3} \mathrm{He}+{ }^{\text {nat }} \mathrm{Ag}$ reaction at $65 \mathrm{MeV}$ bombarding energy is shown in Fig. 7.10. The weight function $S(\epsilon)$ is chosen to be the fit shown in Fig. 7.1. Shown in the lower panel in Fig. 7.10 are the measured alpha spectrum and the linear combination of the orthogonal functions (Eq. 7.7) up to the $10^{\text {th }}$ order. This linear combination of the orthogonal functions indeed represents the spectrum in all its details. The percentage residuals of the 


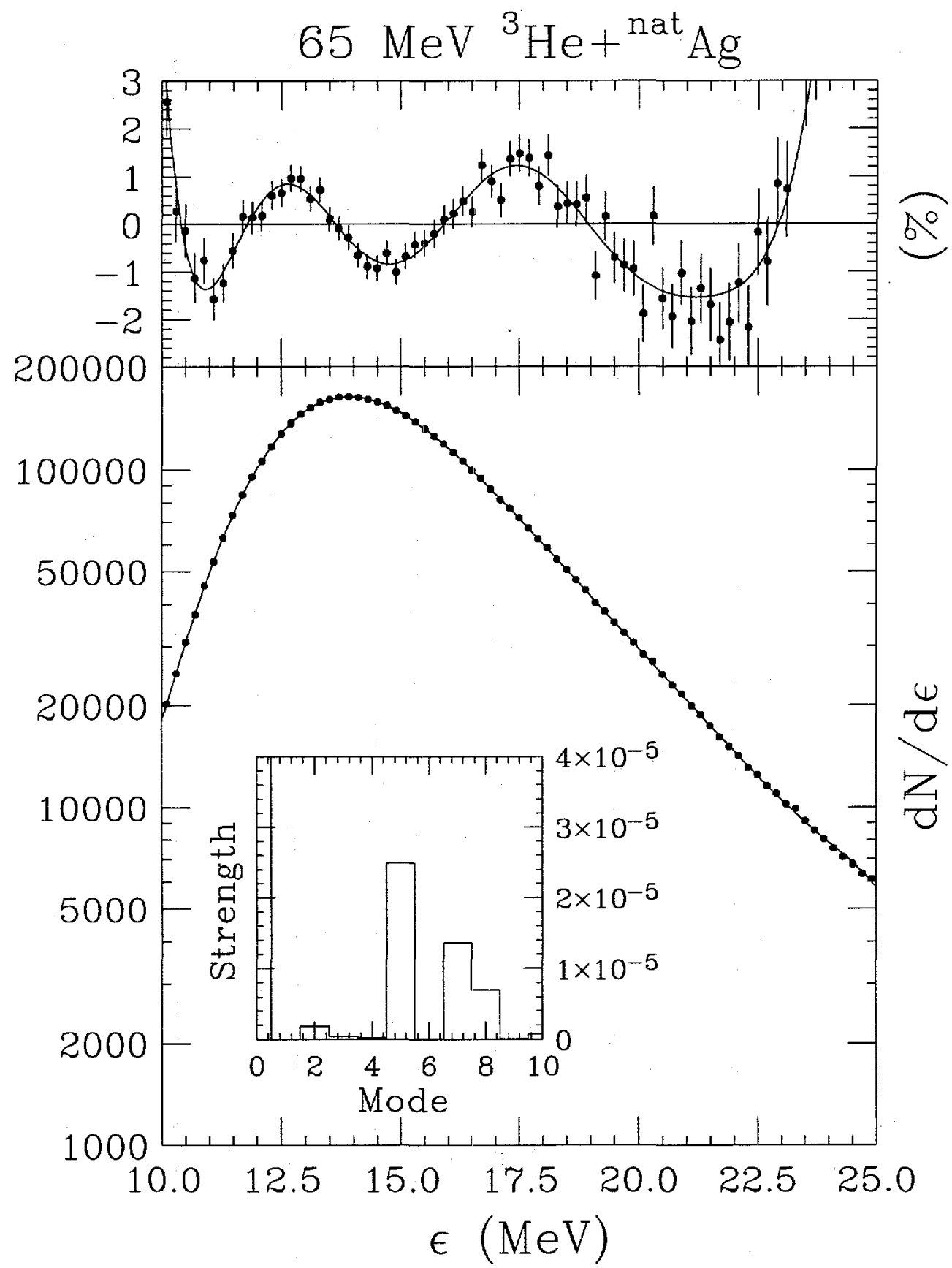

Figure 7.10: Lower panel: The measured C.M. $\alpha$-particle energy spectra $(0)$ and the linear combination of the orthogonal functions (Eq. 7.7) (solid lines), for the ${ }^{3} \mathrm{He}+{ }^{\text {nat }} \mathrm{Ag}$ reaction at $65 \mathrm{MeV}$ beam energy. The inserted figure shows the strength $s_{n}$ of the $n^{\text {th }}$ order as defined in Eq. 7.10 plotted against the order $n$. Upper panel: The dots are the percentage difference between the experimental data and the fit with Eq. 7.3. The error bars represent the statistical errors of the experimental data. The solid line is the percentage difference between the combination of the orthogonal functions and the fit. 
fit, and the percentage difference between the linear combination of the orthogonal functions and the fit, are plotted in the upper panel of the figure. The nearly perfect match between the two percentage differences shows also the excellent quality of the polynomials representation. The strength $s_{n}$ as defined in Eq. 7.10 is plotted against the order $n$ in the insert inside the lower panel. We see that indeed $c_{0}\left(s_{0} \approx 1\right)$ takes up the bulk of the spectrum, and that only a few of the coefficients $c_{n}$ of small magnitude suffice to exhaust the modulation.

Shown in the lower panels of sextants in Fig. 7.11 are the measured centerof-mass $\alpha$-energy spectra and the linear combination of the orthogonal functions, for the ${ }^{3} \mathrm{He}+{ }^{\text {nat }} \mathrm{Ag}$ reactions at bombarding energies of 55, 65, 75, 85, 95, and $110 \mathrm{MeV}$. The weight functions are chosen to be the corresponding fits shown in Fig. 7.2. Plotted in the upper panels of sextants are the percentage residuals of the fits, and the percentage differences between the linear combination of the orthogonal functions and the fits. We see that both the spectra and the residuals of the fits are excellently represented by a combination of the orthogonal functions. In Fig. 7.12 the strength $s_{n}$ is plotted against the order $n$. At all bomdarding energies, the $0^{\text {th }}$ order amplitudes $c_{0}$ take up the bulk of the spectra $\left(s_{n} \approx 1\right)$, and only a few coefficients $c_{n}$ of small magnitude suffice to exhaust the modulations. The amplitudes for the $5^{\text {th }}$ order $\left(c_{5}\right)$ seem to stand out and retain approximately the same magnitude for all bombarding energies.

For the C.M. $\alpha$-energy spectra from the ${ }^{3} \mathrm{He}+{ }^{197} \mathrm{Au}$ reactions at 75,85 , 95, and $110 \mathrm{MeV}$ bombarding energies, the linear combination of the orthogonal 


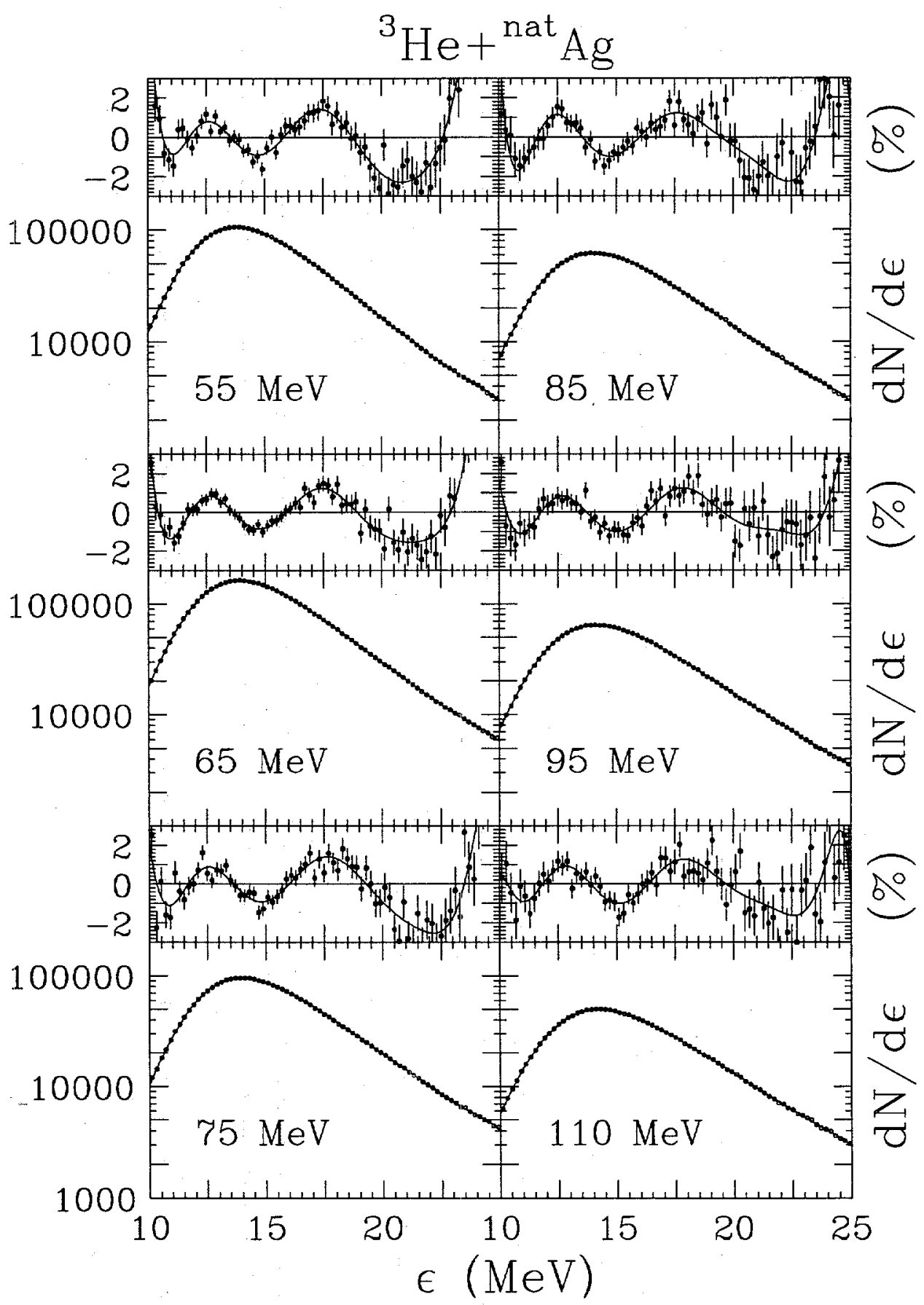

Figure 7.11: Lower panels: The measured C.M. $\alpha$-particle energy spectra $(0)$ and the linear combination of the orthogonal functions (Eq. 7.7) (solid lines), for the ${ }^{3} \mathrm{He}+{ }^{\text {nat }} \mathrm{Ag}$ at six different bombarding energies. Upper panels: The dots are the percentage differences between the experimental data and the fits with Eq. 7.3. The error bars represent the statistical errors of the experimental data. The solid lines are the percentage differences between the combination of the orthogonal functions and the fits. 


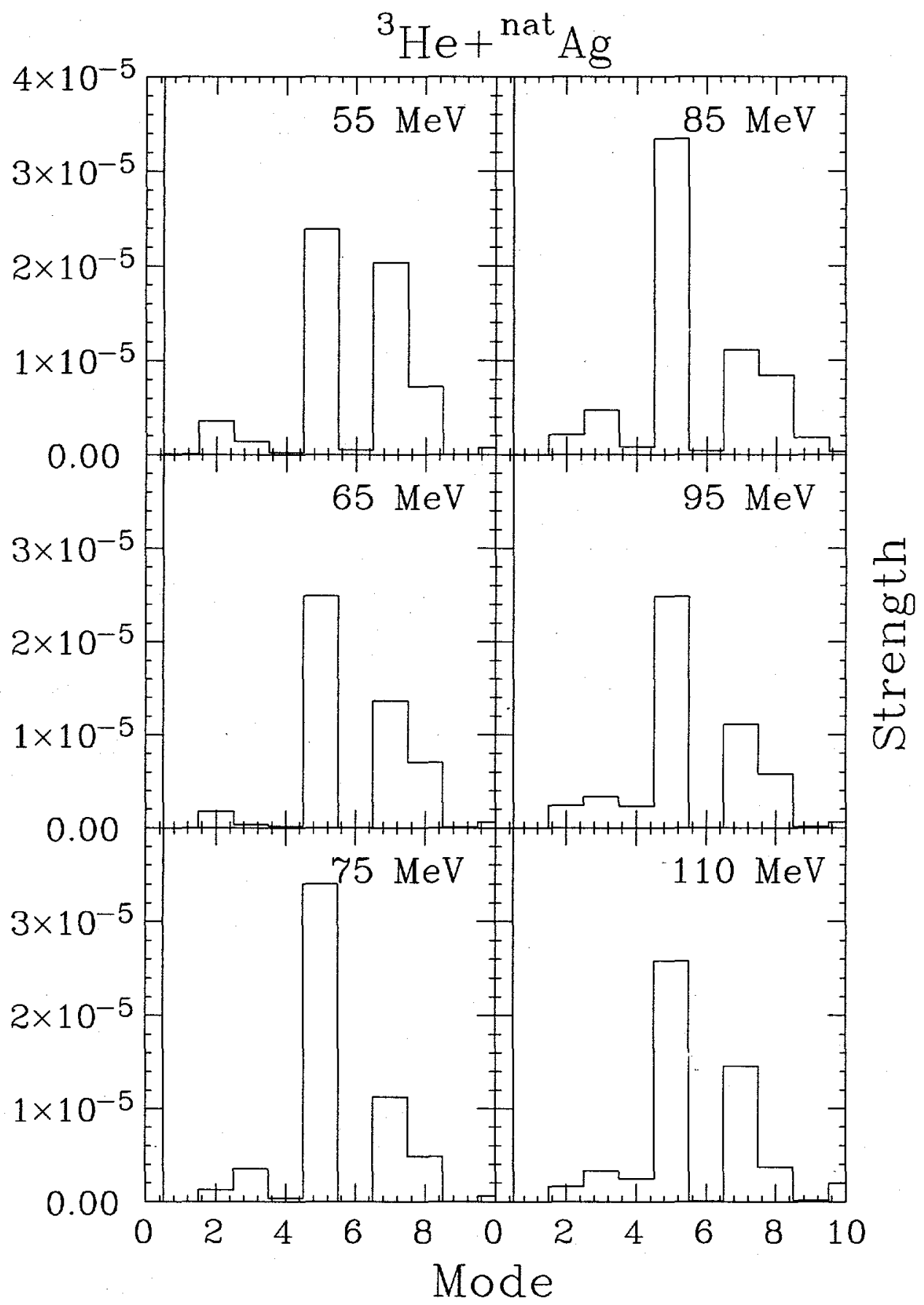

Figure 7.12: The strength $s_{n}$ of the $n^{\text {th }}$ order as defined in Eq. 7.10 is plotted against the order $n$, for the ${ }^{3} \mathrm{He}+{ }^{\text {nat }} \mathrm{Ag}$ reactions at $55,65,75,85,95$, and $110 \mathrm{MeV}$ bombarding energies. 


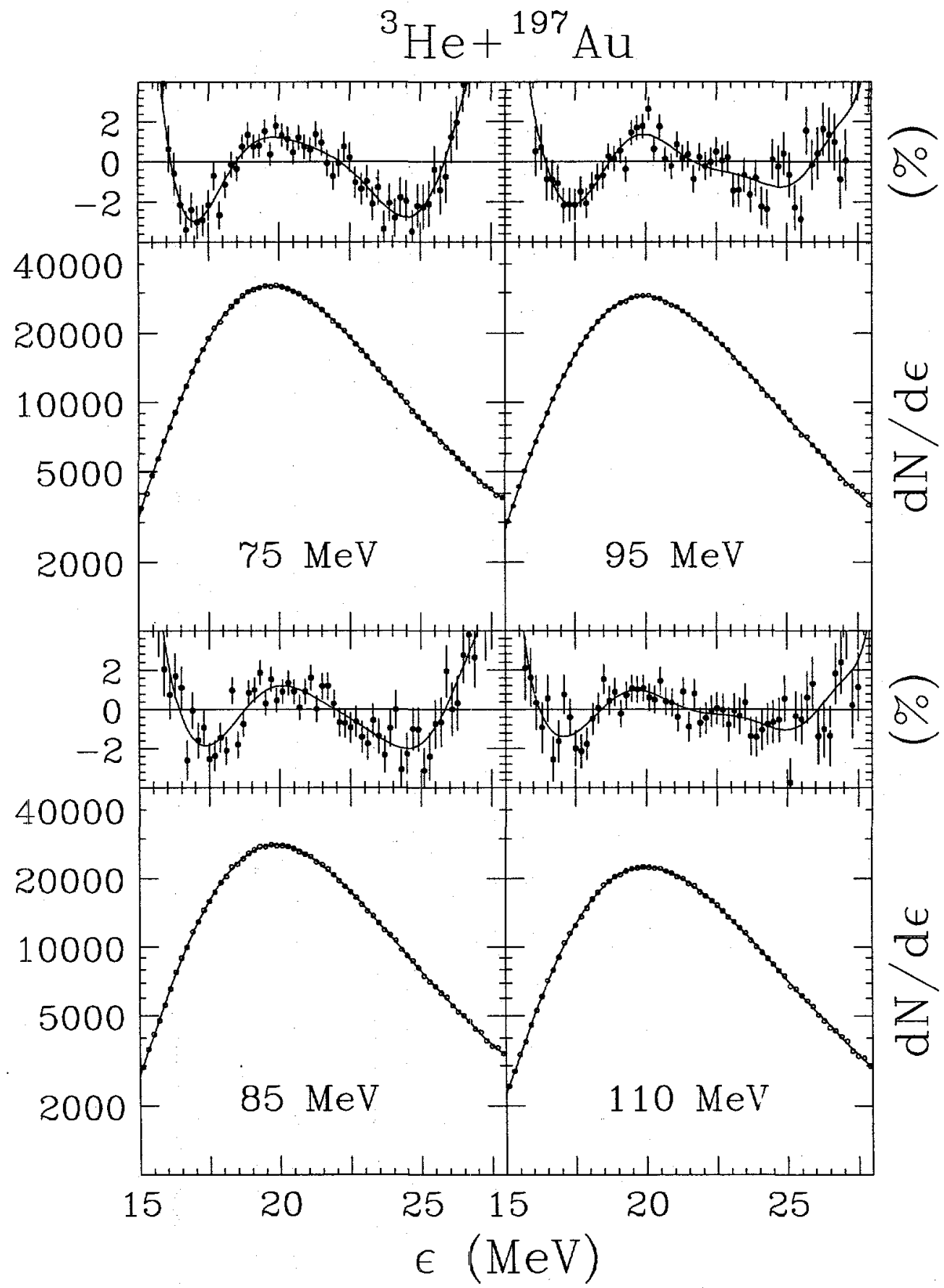

Figure 7.13: Lower panels: The measured C.M. $\alpha$-particle energy spectra ( 0 ) and the linear combination of the orthogonal functions (Eq. 7.7) (solid lines), for the ${ }^{3} \mathrm{He}+{ }^{197} \mathrm{Au}$ at four different bombarding energies. Upper panels: The dots are the percentage differences between the experimental data and the fits with Eq. 7.3. The error bars represent the statistical errors of the experimental data. The solid lines are the percentage differences between the combination of the orthogonal functions and the fits. 


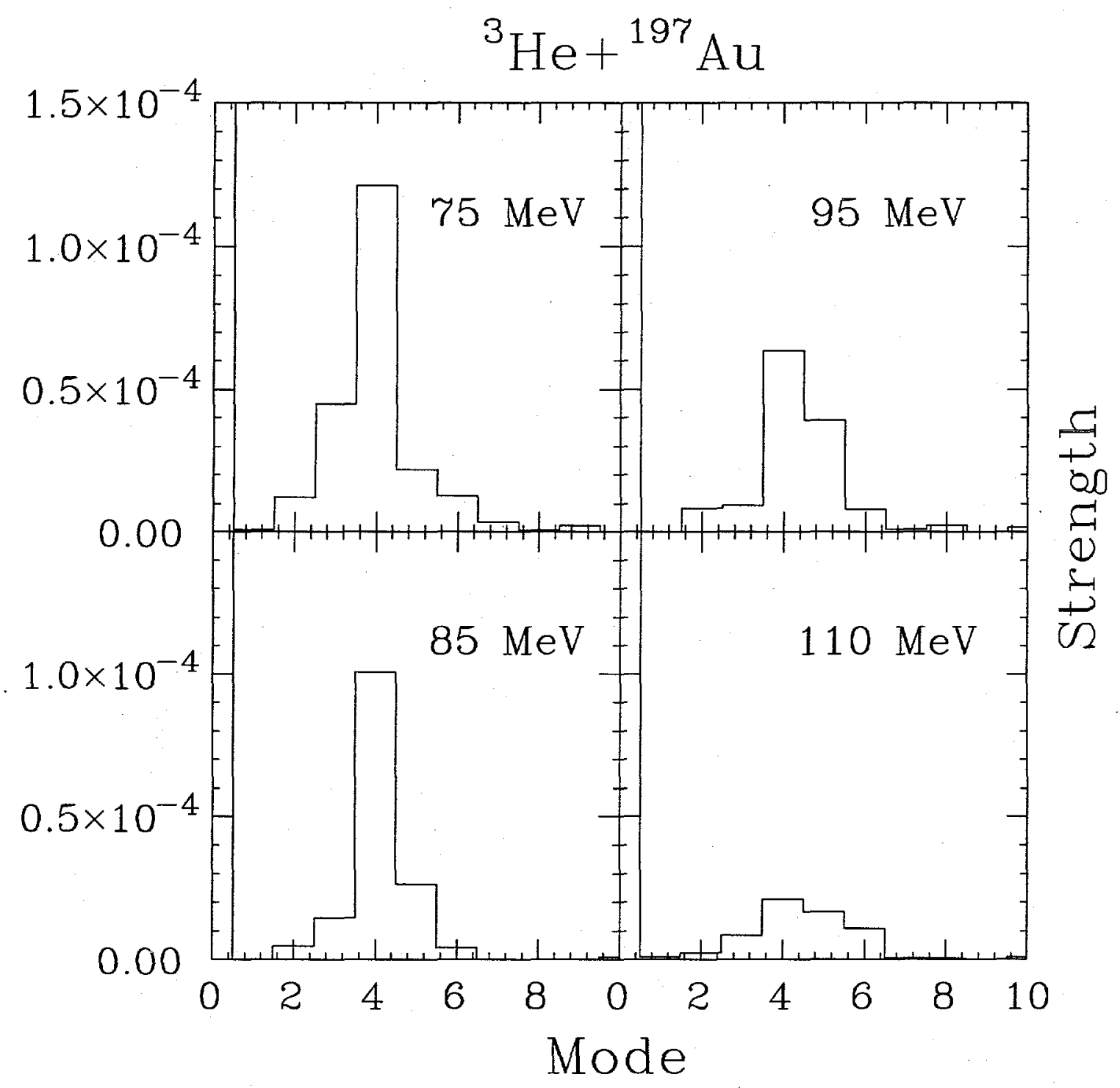

Figure 7.14: The strength $s_{n}$ of the $n^{\text {th }}$ order as defined in Eq. 7.10 is plotted against the order $n$, for the ${ }^{3} \mathrm{He}+{ }^{197} \mathrm{Au}$ reactions at $75,85,95$, and $110 \mathrm{MeV}$ bombarding energies. 
functions also represents very well both the spectra and the residuals of the fits, as shown in Fig. 7.13. The corresponding strength $s_{n}$ is plotted versus the order $n$ in Fig. 7.14. As expected, the strength $s_{0}$ for the $0^{\text {th }}$ order is approximately equal to 1 , and only a few coefficients $c_{n}$ of small magnitude suffice to exhaust the residuals of the fits.

The orthogonal polynomials analysis presented above provides us a way to quantitatively describe both the measured spectra and the observed modulations in the spectra (see Fig. 7.11). It also allows us to extract information on the strength of a mode represented by a polynomial of certain order $n$, although at this stage what physical meaning that may be attached to the polynomials is unclear. In general, analysis schemes of this sort should be useful to pick up signals in noisy environments.

\subsection{An Alternative Approach}

Although the orthogonal polynomials analysis works excellently in describing quantitatively both the measured spectra and the observed modulations, it falls short of convincing us that the observed modulations are really physical. To believe the modulations observed in the residuals of the fits still requires the assumption that the fits truthfully represent the unmodulated spectral shape.

It becomes clear that new ways to identify structrual modulations without relying on a fit with certain spectral shape must be developed.

One alternative approach is to use a smoothing procedure, which retains 
the long-range variations but removes the short-range modulations, to provide the unmodulated background spectral shape. Strutinski has developed a procedure based on a Gaussian smoothing function, to obtain smoothed shell model level densities [Stru 67]. The Strutinski procedure has also been generalized to retain higher-order (up to the $7^{\text {th }}$ order) variations in the smoothed shell model level densities [Nils 69, Tsan 69, More 72b]. Using the generalized Strutinski procedure, the smoothed spectrum $F_{s}(\epsilon)$ can be written as:

$$
F_{s}(\epsilon)=\frac{1}{\sqrt{\pi}} \int F\left(\epsilon^{\prime}\right) f_{k}\left(\frac{\epsilon^{\prime}-\epsilon}{\xi}\right) \exp \left[-\left(\frac{\epsilon^{\prime}-\epsilon}{\xi}\right)^{2}\right] d\left(\frac{\epsilon^{\prime}}{\xi}\right)
$$

where $F(\epsilon)$ is the original spectrum to be smoothed; $\xi$ is the width of the Gaussian smoothing function. The function $f_{k}$ is chosen in such a way that the smoothed spectrum retains the long-range variations up to a fixed order. Choosing the function $f_{k}$ as [Nils 69, Tsan 69, More 72b]:

$$
f_{k}(u)=\sum_{n=0}^{k} \frac{(-1)^{n}}{2^{2 n} n !} H_{2 n}(u)
$$

where $u=\frac{\epsilon^{\prime}-\epsilon}{\xi}$, and $H_{2 n}$ are the Hermite polynomials of even order, the smoothed spectrum then retains the long-range variations up to the $(2 k+1)^{\text {th }}$ order (see Appendix A).

This smoothing procedure works excellently to obtain long-range features. However, in the current case, this smoothing procedure does not help, since the width of the modulation is comparable to the feature scale of the spectra. The modulation either gets no chance to stand out (with small $\xi$ ), or entangles with spectral shape itself (with large $\xi$ ). 


\subsection{Conclusion}

The search for evidence for the existence of complex particles as independent particles inside a nucleus is still an ongoing effort. Tentative evidence is presented in this part for structural modulations of alpha particles similar to those expected for optical potentials. The presented structural modulations were observed in the residuals of the fits to the measured alpha spectra with a smooth spectral shape. The spectral shape used in the fitting is shown to represent alpha spectra to an excellent precision, although this is not sufficient to convince one that the modulations, which appear in the residuals of the fits, are a measure of $\alpha$-particle resonances in a potential well.

An analysis procedure based on orthogonal polynomials has been developed, to quantitatively describe the residuals of the fits. This procedure allows one to

extract information on the strength of a mode represented by a polynomial of a certain order. However, the physical meaning of the polynomials, which are generated by using the spectral shape as their weight function, is unclear.

It seems still a long way to reach definite conclusions regarding the physical reality of the observed modulations, thus the existence of complex particles as independent particles inside a nucleus. 


\section{Bibliography}

[Back 97] B. B. Back, D. J. Hofman, and V. Nanal, Phys. Rev. Lett. 79, 4294 (1997).

[Bass 74] R. Bass, Nucl. Phys. A231, 45 (1974); Phys. Lett. 47B, 139 (1973). Phys. Rev. Lett. 39, 265 (1977).

[Bohr 39] N. Bohr and J. A. Wheeler, Phys. Rev. 56, 426 (1939).

[Char 90] R. J. Charity, K. X. Jing, D. R. Bowman, M. A. McMahan, G. J. Wozniak, L. G. Moretto, N. Colonna, G. Guarino, A. Pantaleo, L. Fiore, A. Gobbi and K. D. Hildenbrand, Nucl. Phys. A511, 59 (1990).

[Char 95] R. J. Charity, Phys. Rev. C 51, 217 (1995).

[Char 97] R. J. Charity, M. Korolija, D. G. Sarantites, and L. G. Sobotka, Phys. Rev. C 56, 873 (1997).

[Clar 90] D. J. Clark and G. J. Wozniak, Nucl. Instr. and Meth. A295, 34 (1990).

[Deli 91] D. N. Delis, Y. Blumenfeld, D. R. Bowman, N. Colonna, K. Hanold, K. Jing, M. C. Meng, G. F. Peaslee, G. J. Wozniak and L. G. Moretto, Nucl. Phys. A534, 403 (1991). 
[Eric 60] T. Ericson, Adv. in Phys. 9, 425 (1960); and references therein.

[Fesh 54] H. Feshbach, C. Porter, and V. F. Weisskopf, Phys. Rev. 96, 448 (1954).

[Frob 93] P. Fröbrich, I. I. Gontchar, and N. D. Mavlitov, Nucl. Phys. A556, 281 (1993).

[Gran 83] P. Grangé and H. A. Weidenmüller, Phys. Lett. 96B, 26 (1980).

[Han 89] H. Y. Han, K. X. Jing, E. Plagnol, D. R. Bowman, R. J. Charity, L. Vinet, G. J. Wozniak and L. G. Moretto, Nucl. Phys. A492, 138 (1989).

[Haus 52] W. Hauser and H. Feshbach, Phys. Rev. 87, 366 (1952).

[Hill 53] D. L. Hill and J. A. Wheeler, Phys. Rev. 89, 1102 (1953).

[Hils 92] D. Hilscher and H. Rossner, Ann. Phys. Fr. 17, 471 (1992); and references therein.

[Huiz 72] J. R. Huizenga and L. G. Moretto, Anilu. Rev. Nucl. Sci. 22, 427 (1972); and references therein.

[Jing 99] K. X. Jing, L. G. Moretto, A. C. Veeck, N. Colonna, I. Lhenry, K. Tso, K. Hanold, W. Skulski, Q. Sui, and G. J. Wozniak, Nucl. Phys. A645, 203 (1999).

[Keho 92] W. L. Kehoe, A. C. Mignerey, A. Moroni, I. Iori, G. F. Peaslee, N. Colonna, K. Hanold, D. R. Bowman, L. G. Moretto, M. A. McMahan, J. T. Walton and G. J. Wozniak, Nucl. Instr. and Meth. A311, 258 (1992). 
[Khod 66] A. Khodai-Joopari, Ph.D. thesis, Univ. of California at Berkeley, 1966.

[Kram 40] H. A. Kramers, Physica VII, No.4, 284 (1940).

[Lest 93a] J. P. Lestone, Phys. Rev. Lett. 70, 2245 (1993);

[Lest 93b] J. P. Lestone, J. R. Leigh, L. O. Newton, D. J. Hinde, J. X. Wei, J. X. Chen, S. Elfström, M. Zielinska-Pfabé, Nucl. Phys. A559, 277 (1993).

[Macf 78] M. H. Macfarlane and S. C. Pieper, Optical model code PTolemy, (Argonne National Laboratory, ANL-76-11, 1978).

[McMa 86] M. A. McMahan, G. J. Wozniak, C. M. Lyneis, D. R. Bowman, R. J. Charity, Z. H. Liu, L. G. Moretto, W. L. Kehoe, A. C. Mignerey and M. N. Namboodiri, Nucl. Instr. and Meth. A253, 1 (1986).

[Micr 97] MicroMatter Co., Deer Habor, Washington, USA, private communication, (1997).

[Möll 93] P. Möller, J. R. Nix, W. D. Myers, and W. J. Swiatecki, Los Alamos National Laboratory Report No. LA-UR-93-3083, 1993.

[More 72a] L. G. Moretto, S. G. Thompson, J. Routti, and R. C. Gatti, Phys. Lett. 38B, (1972) 471.

[More 72b] L. G. Moretto, Nucl. Phys. A182, 641 (1972).

[More 74] L. G. Moretto, "Fission Probabilities in Lighter Nuclei: A theoretical and experimental investigation of the shell and pairing effects in fission- 
ing nuclei," Physics and Chemistry of Fission 1973, (Vienna: International Atomic Energy Agency, 1974) I: p.329.

[More 75] $\quad$ L. G. Moretto, Nucl. Phys. A.247, (1975) 211.

[More 87] L. G. Moretto and D. R. Bowman, Lawrence Berkeley Laboratory, Report LBL-23228 (1987).

[More 95a] L. G. Moretto, K. X. Jing, and G. J. Wozniak, Phys. Rev. Lett. 74, 3557 (1995).

[More 95b] L. G. Moretto, K. X. Jing, R. Gatti, R. P. Schmitt, and G. J. Wozniak, Phys. Rev. Lett. 75, 4186 (1995).

[More 97a] L. G. Moretto, K. X. Jing, G. Gatti, Th. Rubehn, G. J. Wozniak, and R. P. Schmitt, Phys. Rev. Lett. 79, 4295 (1997).

[More 97b] $\quad$ L. G. Moretto, K. X. Jing, L. Phair, and G. J. Wozniak, J. Phys. G: Nucl. Part. Phys. 23, 1323 (1997).

[Mort 97] C. R. Morton, A. Buda, P. Paul, N. P. Shaw, J. R. Beene, N. Gan, M. L. Halbert, D. W. Stracener, R. L. Varner, M. Theonnessen, P. Thirolf, and I. Dioszegi, J. Phys. G: Nucl. \& Part. Phys. 23, 1383 (1997).

[Myer 65] W. D. Myers and W. J. Swiatecki, Lawrence Radiation Laboratory, UCRL$11980,(1965)$

[Myer 94] W. D. Myers and W. J. Swiatecki, Lawrence Berkeley National Laboratory, LBL-36803, (1994). 
[Myer 96] W. D. Myers and W. J. Swiatecki, Nucl. Phys. A601, (1996) 141.

[Nato 90] J. B. Natowitz, M. Gonin, M. Gui, K. Hagel, Y. Lou, D. Utley, and R. Wada, Phys. Lett. 247B, 242 (1990).

[Newt 89] J. O. Newton, Pramãna - J. Phys. 33, 175 (1989); and references therein.

[Nils 69] S. G. Nilsson, C. F. Tsang, A. Sobiczewski, Z. Szymanski, S. Wycech, C. Gustafson, I. L. Lamm, P. Moeller, and B. Nilsson, Nucl. Phys. A131, $1(1969)$.

[Park 91] W. E. Parker, M. Kaplan, D. J. Moses, G. La Rana, D. Logan, R. Lacey, J. M. Alexander, D. M. de Castro Rizzo, P. DeYoung, R. J. Welberry, and J. T. Boger, Phys. Rev. C 44, 774 (1991).

[Paul 94] P. Paul and M. Thoennessen, Ann. Rev. Nucl. Part. Sci. 44, 65 (1994); and references therein.

[Pres 92] W. H. Press, S. A. Teukolski, W. T. Vettering, and B. P. Flannery, Numerical Recipes in C: The Art of Scientific Computing, 2nd ed., Cambridge Univ. Press, Cambridge, (1992).

[Rose 57] N. Rosenzweig, Phys. Rev. 108, 817 (1957).

[Rube 96] Th. Rubehn, K. X. Jing, L. G. Moretto, L. Phair, K. Tso, and G. J. Wozniak, Phys. Rev. C 54, 3062 (1996).

[Rube 97] Th. Rubehn, G. J. Wozniak, L. Phair, L. G. Moretto, and Kin M. Yu, Nucl. Instr. Meth. A387, 328 (1997). 
[Sano 63] M. Sano and S. Yamasaki, Prog. Theor. Phys. 29, 397 (1963).

[Sans 59] G. Sansone, Orthogonal Functions, Interscience Publishers, Inc., New York, (1959).

[Stic 74] J. D. Stickler and K. J. Hofstetter, Phys. Rev. C 9, 1064 (1974).

[Stru 67] V. M. Strutinski, Nucl. Phys. A95, 420 (1967).

[Swia 82] W. J. Swiatecki, Nucl. Phys. A376, 275 (1982); Phys. Scripta 24, 113 (1981); S. Bjørnholm and W. J. Swiatecki, Nucl. Phys. A391, 471 (1982).

[Tsan 69] C. F. Tsang, Ph.D Thesis, UCRL-18899, (1969).

[Vand 73j R. Vandenbosch and J. R. Huizenga, Nuclear Fission, Academic Press, New York, (1973); and references therein.

[Vaz 84] L. C. Vaz and J. M. Alexander, Z. Phys. A 318, 231 (1984).

[Walt 90] J. T. Walton, H. A. Sommer, G. J. Wozniak, G. F. Peaslee, D. R. Bowman, W. L. Kehoe and A. Moroni, IEEE Trans. Nucl. Sci. 37, 1578 (1990).

[Weis 37] V. Weisskopf, Phys. Rev. 52, 295 (1937).

[Wign 38] E. Wigner, Trans. Faraday Soc. 34, part 1, 29 (1938). 


\section{Appendix A}

\section{Generalized Strutinski Smoothing}

\section{Procedure}

Using the generalized Strutinski smoothing procedure [Nils 69, Tsan 69, More $72 \mathrm{~b}]$, the smoothed function $F_{s}(x)$ can be written as:

$$
F_{s}(x)=\frac{1}{\sqrt{\pi}} \int_{-\infty}^{\infty} F\left(x^{\prime}\right) f_{k}\left(\frac{x^{\prime}-x}{\xi}\right) \exp \left[-\left(\frac{x^{\prime}-x}{\xi}\right)^{2}\right] d\left(\frac{x^{\prime}}{\xi}\right)
$$

where $F(x)$ is a function to be smoothed, and $\xi$ is the width of the Gaussian smoothing function. The correction function $f_{k}\left(u=\frac{x^{\prime}-x}{\xi}\right)$ is so chosen that the smoothed function retains the long range variations up to a fixed order. In the following it will be demonstrated that, choosing $f_{k}$ to be [More 72b]

$$
f_{k}(u)=\sum_{n=0}^{k} \frac{(-1)^{n}}{2^{2 n} n !} H_{2 n}(u)
$$

where $k$ is a positive integer and $H_{2 n}(u)$ are the Hermite polynomials of even order, the smoothed function $F_{s}(x)$ retains the long range variations up to the $(2 k+1)^{\text {th }}$ 
order. Equivalently, it is to show that, for $m=0,1,2, \cdots,(2 k+1)$,

$$
x^{m}=\frac{1}{\sqrt{\pi}} \int_{-\infty}^{\infty}(\xi u+x)^{m}\left(\sum_{n=0}^{k} \frac{(-1)^{n}}{2^{2 n} n !} H_{2 n}(u)\right) \exp \left(-u^{2}\right) d u
$$

First, it is easily verified that, for $m=0,1, \cdots,(2 k+1)$, Equation A.3 is valid if $k=0,1,2,3$ (see also refs. [Nils 69, Tsan 69]).

Now, given that, for $m=0,1,2, \cdots,(2 k-1)$,

$$
x^{m}=\frac{1}{\sqrt{\pi}} \int_{-\infty}^{\infty}(\xi u+x)^{m}\left(\sum_{n=0}^{k-1} \frac{(-1)^{n}}{2^{2 n} n !} H_{2 n}(u)\right) \exp \left(-u^{2}\right) d u,
$$

we show that Eq. A.3 is valid for $m=0,1,2, \cdots,(2 k+1)$. Notice that, for $m=0$, $1,2, \cdots,(2 k-1)$

$$
\begin{aligned}
& \frac{1}{\sqrt{\pi}} \int_{-\infty}^{\infty}(\xi u+x)^{m}\left(\sum_{n=0}^{k} \frac{(-1)^{n}}{2^{2 n} n !} H_{2 n}(u)\right) \exp \left(-u^{2}\right) d u \\
& =\frac{1}{\sqrt{\pi}} \int_{-\infty}^{\infty}(\xi u+x)^{m}\left(\sum_{n=0}^{k-1} \frac{(-1)^{n}}{2^{2 n} n !} H_{2 n}(u)\right) \exp \left(-u^{2}\right) d u \\
& \quad+\frac{1}{\sqrt{\pi}} \int_{-\infty}^{\infty}(\xi u+x)^{m} \frac{(-1)^{k}}{2^{2 k} k !} H_{2 k}(u) \exp \left(-u^{2}\right) d u \\
& =x^{m}+\frac{1}{\sqrt{\pi}} \int_{-\infty}^{\infty}\left(\sum_{n=0}^{m} a_{n}(x, \xi) H_{n}(u)\right) \frac{(-1)^{k}}{2^{2 k} k !} H_{2 k}(u) \exp \left(-u^{2}\right) d u \\
& =x^{m}
\end{aligned}
$$

where the orthogonality condition of the Hermite polynomials

$$
\int_{-\infty}^{\infty} H_{n}(u) H_{m}(u) \exp \left(-u^{2}\right) d u= \begin{cases}0, & n \neq m \\ 2^{n} n ! \sqrt{\pi}, & n=m\end{cases}
$$

has been used. Thus, for $m=2 k-1$ in particular, Eq. A. 5 becomes

$$
x^{2 k-1}=\frac{1}{\sqrt{\pi}} \int_{-\infty}^{\infty}(\xi u+x)^{2 k-1}\left(\sum_{n=0}^{k} \frac{(-1)^{n}}{2^{2 n} n !} H_{2,2}(u)\right) \exp \left(-u^{2}\right) d u
$$


Next we show that Eq. A.3 is valid for $m=2 k$ and $2 k+1$. By Eq. A.7,

$$
\begin{aligned}
x^{2 k}= & \int_{0}^{x} 2 k x^{2 k-1} d x \\
= & \frac{1}{\sqrt{\pi}} \int_{-\infty}^{\infty}\left[\int_{0}^{x} 2 k(\xi u+x)^{2 k-1} d x\right]\left(\sum_{n=0}^{k} \frac{(-1)^{n}}{2^{2 n} n !} H_{2 n}(u)\right) \exp \left(-u^{2}\right) d u \\
= & \frac{1}{\sqrt{\pi}} \int_{-\infty}^{\infty}(\xi u+x)^{2 k}\left(\sum_{n=0}^{k} \frac{(-1)^{n}}{2^{2 n} n !} H_{2 n}(u)\right) \exp \left(-u^{2}\right) d u \\
& -\frac{1}{\sqrt{\pi}} \int_{-\infty}^{\infty}(\xi u)^{2 k}\left(\sum_{n=0}^{k} \frac{(-1)^{n}}{2^{2 n} n !} H_{2 n}(u)\right) \exp \left(-u^{2}\right) d u \\
= & \frac{1}{\sqrt{\pi}} \int_{-\infty}^{\infty}(\xi u+x)^{2 k}\left(\sum_{n=0}^{k} \frac{(-1)^{n}}{2^{2 n} n !} H_{2 n}(u)\right) \exp \left(-u^{2}\right) d u
\end{aligned}
$$

where the identity

$$
\int_{-\infty}^{\infty} u^{2 k}\left(\sum_{n=0}^{k} \frac{(-1)^{n}}{2^{2 n} n !} H_{2 n}(u)\right) \exp \left(-u^{2}\right) d u=0, \quad(k \geq 1)
$$

has been used. The proof of this identity will be given later. In the same way,

$$
\begin{aligned}
x^{2 k+1}= & \int_{0}^{x}(2 k+1) x^{2 k} d x \\
= & \frac{1}{\sqrt{\pi}} \int_{-\infty}^{\infty}\left[\int_{0}^{x}(2 k+1)(\xi u+x)^{2 k} d x\right]\left(\sum_{n=0}^{k} \frac{(-1)^{n}}{2^{2 n} n !} H_{2 n}(u)\right) \exp \left(-u^{2}\right) d u \\
= & \frac{1}{\sqrt{\pi}} \int_{-\infty}^{\infty}(\xi u+x)^{2 k+1}\left(\sum_{n=0}^{k} \frac{(-1)^{n}}{2^{2 n} n !} H_{2 n}(u)\right) \exp \left(-u^{2}\right) d u \\
& -\frac{1}{\sqrt{\pi}} \int_{-\infty}^{\infty}(\xi u)^{2 k+1}\left(\sum_{n=0}^{k} \frac{(-1)^{n}}{2^{2 n} n !} H_{2 n}(u)\right) \exp \left(-u^{2}\right) d u \\
= & \frac{1}{\sqrt{\pi}} \int_{-\infty}^{\infty}(\xi u+x)^{2 k+1}\left(\sum_{n=0}^{k} \frac{(-1)^{n}}{2^{2 n} n !} H_{2 n}(u)\right) \exp \left(-u^{2}\right) d u,
\end{aligned}
$$

where

$$
\int_{-\infty}^{\infty} u^{2 k+1}\left(\sum_{n=0}^{k} \frac{(-1)^{n}}{2^{2 n} n !} H_{2 n}(u)\right) \exp \left(-u^{2}\right) d u=0
$$

since the integrand is an odd function. 
Now the proof of Eq. A.9 is given to finish the proof of Eq. A.3. It is easily verified that Eq. A.9 is true for $k=1$ and 2 . We show in the following that Eq. A.9 holds for any $k(k \geq 1)$. Notice that $u^{2 k-2}$ can always be expressed as

$$
u^{2 k-2}=\sum_{n=0}^{k-1} a_{n} H_{2 n}(u),
$$

where $a_{n}$ are the coefficients properly chosen to make the identity. Thus, by using the recurrence formula of the Hermite polynomials

$$
H_{n+1}(u)+2 u H_{n}(u)+2 n H_{n-1}(u)=0
$$

one gets

$$
\begin{aligned}
4 u^{2 k}= & 4 u^{2} u^{2 k-2}=4 u^{2} \sum_{n=0}^{k-1} a_{n} H_{2 n}(u) \\
= & a_{0}\left(H_{2}(u)+2 H_{0}(u)\right) \\
& +\sum_{n=1}^{k-1} a_{n}\left(H_{2 n+2}(u)+(8 n+2) H_{2 n}(u)+8 n(2 n-1) H_{2 n-2}(u)\right) .
\end{aligned}
$$

Therefore,

$$
\begin{aligned}
& \int_{-\infty}^{\infty} 4 u^{2 k}\left(\sum_{n=0}^{k} \frac{(-1)^{n}}{2^{2 n} n !} H_{2 n}(u)\right) \exp \left(-u^{2}\right) d u \\
& =\int_{-\infty}^{\infty} a_{0}\left(H_{2}(u)+2 H_{0}(u)\right)\left(\sum_{n=0}^{k} \frac{(-1)^{n}}{2^{2 n} n !} H_{2 n}(u)\right) \exp \left(-u^{2}\right) d u \\
& \quad+\int_{-\infty}^{\infty}\left[\sum_{n=1}^{k-1} a_{n}\left(H_{2 n+2}(u)+(8 n+2) H_{2 n}(u)+8 n(2 n-1) H_{2 n-2}(u)\right)\right] \\
& \quad\left(\sum_{n=0}^{k} \frac{(-1)^{n}}{2^{2 n} n !} H_{2 n}(u)\right) \exp \left(-u^{2}\right) d u \\
& =0
\end{aligned}
$$

where the integrals are readily evaluated by using the orthogonality equation A.6. QED. 


\section{Appendix B}

\section{Orthogonal Polynomials Analysis}

\section{and a $\mathrm{C}++$ Code}

A system of polynomials $P_{n}(x)$ :

$$
P_{n}(x)=a_{n} x^{n}+a_{n}^{\prime} x^{n-1}+\cdots, \quad(n=0,1,2, \cdots),
$$

is called orthogonal on the interval $[a, b]$ with respect to the weight function $w(x)$, if

$$
\int_{a}^{b} w(x) P_{n}(x) P_{m}(x) d x=\left\{\begin{array}{cc}
0, & n \neq m \\
h_{n}, & n=m .
\end{array}\right.
$$

The weight function $w(x)(w(x) \geq 0, x \in[a, b])$ determines the system $P_{n}(x)$ up to a constant factor in each polynomial. The specification of these factors is referred to as standardization. The usual way to standardise is called normalization by specifying $h_{n}=1$.

One example of orthogonal polynomials is the Legendre polynomials where 
$w(x)=1, x \in[-1,1]$. The Legendre polynomials are widely used to represent the angular distributions of products in nuclear reactions.

Another example of orthogonal polynomials is the Hermite polynomials where $w(x)=\exp \left(-x^{2}\right), x \in[-\infty, \infty]$. The Hermite polynomials can be used to represent the wave functions of a harmonic oscillator.

\section{B.1 Generation of Orthogonal Polynomials for An Arbitrary Weight Function}

Given a weight function $w(x)$ defined in $[a, b]$ that the following integrals (called moments) exist and are finite

$$
\alpha_{n}=\int_{a}^{b} w(x) x^{n} d x, \quad(n=0,1,2, \cdots)
$$

and that $\alpha_{0}>0$, then it is possible to construct in a unique way, apart from the sign, a sequence of orthogonal polynomials $P_{n}(x)$ with respect to the weight function $w(x)$ in $[a, b]$. This is shown as follows [Sans 59].

If we let

$$
\Phi_{n}(x)=P_{n}(x) / a_{n}=x^{n}-D_{n} x^{n-1}+\cdots
$$

then

$$
\begin{array}{cl}
\Phi_{0}(x)=1 ; & 1=\int_{a}^{b} w(x) a_{0}^{2} d x, \quad 1 / a_{0}^{2}=\alpha_{0} ; \\
\Phi_{1}(x)=x-D_{1} ; & \int_{a}^{b} w(x)\left[x-D_{1}\right] d x=0 ; \\
D_{1}=\alpha_{1} / \alpha_{0} ; & 1 / a_{1}^{2}=\int_{a}^{b} w(x) \Phi_{1}^{2}(x) d x .
\end{array}
$$


To construct the successive polynomials $\Phi_{2}, \Phi_{3}, \cdots$, we notice that for any constants $\mu_{n}, \nu_{n}$ the polynomial

$$
\Phi_{n}(x)=\left(x-\mu_{n}\right) \Phi_{n-1}-\nu_{n} \Phi_{n-2}, \quad(n \geq 2)
$$

has its first coefficient equal to one and is orthogonal to $\Phi_{0}, \Phi_{1}, \cdots, \Phi_{n-3}$. The orthogonality to $\Phi_{n-2}$ with respect to $w(x)$ implies

$$
\int_{a}^{b} x w(x) \Phi_{n-1}(x) \Phi_{n-2}(x) d x=\nu_{n} \int_{a}^{b} w(x) \Phi_{n-2}^{2}(x) d x
$$

the orthogonality to $\Phi_{n-1}$ with respect to $w(x)$ implies

$$
\int_{a}^{b} x w(x) \Phi_{n-1}^{2}(x) d x=\mu_{n} \int_{a}^{b} w(x) \Phi_{n-1}^{2}(x) d x
$$

and the normalization of $a_{n} w^{1 / 2}(x) \Phi_{n}(x)$ in $[a, b]$ implies

$$
a_{n}^{2} \int_{a}^{b} w(x) \Phi_{n}^{2}(x) d x=1
$$

Thus we have the procedure to generate a complete set of orthogonal polynomials with respect to the given weight function $w(x)$.

\section{B.2 Orthogonal Polynomials Representation of Structural Modulations in Alpha-Particle Spectra}

We observe that, in our experimentally measured alpha-particle spectra (see Fig. 7.1, for example), the structural modulations of small magnitudes superimpose on the overall smooth background represented by the fits $S(\epsilon)$ using Eq. 7.3. Choosing the weight function to be $w(\epsilon)=S^{2}(\epsilon)$, the corresponding set of orthogonal 
polynomials $P_{n}(\epsilon)$ can be generated which satisfies the orthogonality condition:

$$
\int_{a}^{b} S^{2}(\epsilon) P_{n}(\epsilon) P_{m}(\epsilon) d \epsilon= \begin{cases}0, & n \neq m \\ 1, & n=m\end{cases}
$$

Then the experimental spectrum $F(\epsilon)$ can be written as a linear combination of orthogonal functions $S(\epsilon) P_{n}(\epsilon)$

$$
F(\epsilon)=\sum_{n} c_{n} S(\epsilon) P_{n}(\epsilon)
$$

where $c_{n}$ is the coefficient which can be considered as the amplitude of a spectral mode corresponding to the $n^{\text {th }}$ order polynomial $P_{n}(\epsilon)$.

The choice of $S^{2}(\epsilon)$ as the weight function guarantees that the coefficient $c_{0}$ of the zero-th order will take up the bulk of the spectrum, and the modulations then appear in the higher order coefficients, hopefully in only one or two. The amplitutes $c_{n}$ can be obtained from the dot product of the experimental spectrum $F(\epsilon)$ with the $n^{\text {th }}$ order orthogonal function $S(\epsilon) P_{n}(\epsilon)$

$$
c_{n}=\int_{a}^{b} F(\epsilon) S(\epsilon) P_{n}(\epsilon) d \epsilon
$$

and the corresponding strength $s_{n}$ can be defined as

$$
s_{n}=c_{n}^{2} / \int_{a}^{b} F^{2}(\epsilon) d \epsilon
$$

By definition we have $\sum s_{n}=1$.

This analysis procedure based on the orthogonal polynomials should allow us to quantitatively describe the modulations in the measured alpha spectra, and extract information such as the strength associated with a spectral mode represented by the polynomial of the order $n$. 


\section{B.3 A C++ Code that Implements the Polynomials}

\section{Analysis Procedure}

A computer code in $\mathrm{C}++$ has been developed to implement the analysis procedure illustrated above. The $\mathrm{C}++$ code package consists of the following four object classes:

- OrthWeightD: Delared in the head file OrthWeightD.h. This class establishes the weight function. It has utility functions that return the values of $w(x), w^{1 / 2}(x)$, and other properties such as the parameters the weight function requires. The class also provides utilities to reset some of its parameters. Its inline member functions are implemented in the head file OrthWeightD.h. Other member functions are implemented in the file OrthWeightD.cxx.

- OrthPolyD: Declared in the head file OrthPolyD.h. OrthPolyD is a subclass of the class OrthWeightD, and inherits all the properties of OrthWeightD. The class OrthPoly $D$ establishes the system of the orthogonal polynomials, with respect to the weight function inherited from OrthWeightD, up to a specified maximum order MaxN_. It has utilities to reset the boundaries of the variable domain $[\mathrm{a}, \mathrm{b}]$ and the maximum order, and reestablish the polynomials upon the resetting of $[\mathrm{a}, \mathrm{b}]$ and/or $\operatorname{MaxN}$. . Its inline member functions are implemented in the head file OrthPolyD.h. Other member functions are implemented in the file OrthPolyD.cxx. 
- Spectrum1D: Declared in the head file Spectrum1D.h. This class establishes the object of one dimensional spectrum which is to be analyzed. It has utility functions that return the values of many aspects of the spectrum properties, such as the counts in a specified channel, the integrated counts in a specified spectrum window, etc.. It has the structure in place for expansion into a full fledged analysis package for one dimensional spectrum, although the full implementation has not been made. For the current analysis, a member function providing access to the spectrum data suffices. The inline member functions are implemented in the head file Spectrum1D.h. Other member functions and non-member functions are implemented in the file Spectrum1D.cxx.

- ModeAmplitudes: Declared in the head file ModeAmplitudes.h. This is the class object in which the orthogonal polynomials analysis is carried out. ModeAmplitudes is a subclass of both the class $\operatorname{OrthPoly} D$ and the class Spectrum $1 D$, and inherits all the properties of OrthPolyD and Spectrum $1 D$. Its inline member functions are implemented in the head file ModeAmplitudes .h. Other member functions are implemented in the file ModeAmplitudes.cxx.

The head files, OrthWeightD.h, OrthPolyD.h, Spectrum1D.h, and ModeAmplitudes.h, should reside in a directory called ORTHPOLY/include, where ORTHPOLY is a symbolic link pointing to the parent directory of include.

The following is a main program (function) which puts everything together, to perform the orthogonal polynomials analysis on the alpha energy spectrum from 
the ${ }^{3} \mathrm{He}+{ }^{\text {nat }} \mathrm{Ag}$ reaction at $65 \mathrm{MeV}$ beam energy. It serves as a sample illustrating how the classes are to be used.

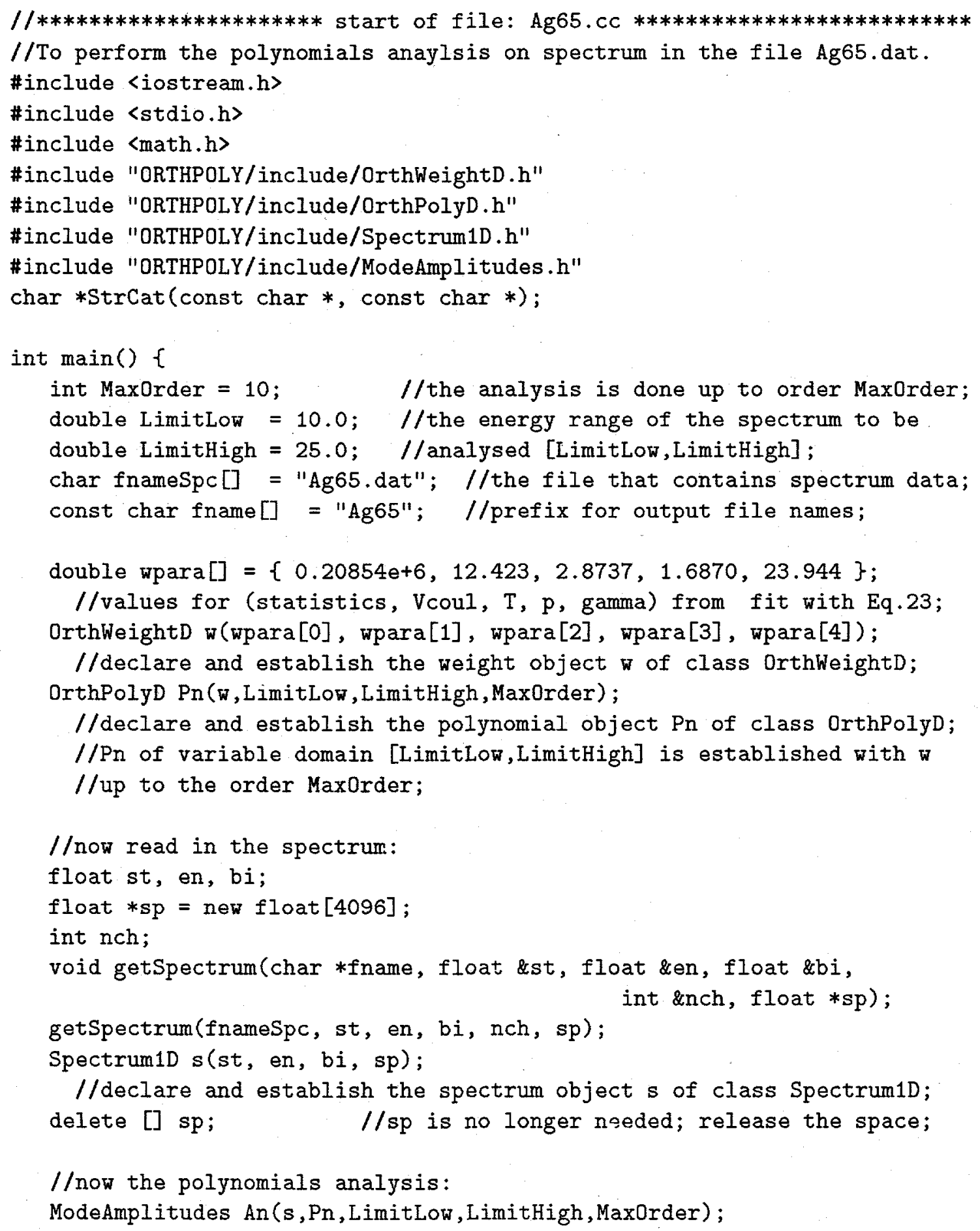

//declare and establish the spectrum object s of class Spectrum1D; delete [] sp; $\quad / / \mathrm{sp}$ is no longer noeded; release the space;

//now the polynomials analysis:

ModeAmplitudes An(s, Pn, LimitLow, LimitHigh, MaxOrder); 
//declare the object An of class ModeAmplitudes; An is established $/ /$ with both $\mathrm{s}$ and $\mathrm{Pn}$, in the domain [LimitLow,LimitHigh], and up to //the order Max0rder;

//print out the results to files:

FILE *Pfit = fopen(StrCat(fname,".fit"), $w+") ;$ //original fit;

FILE *Psum = fopen (StrCat (fname," sum"), "w+"); //sum of orth functions

FILE *Pdif = fopen(StrCat(fname,".dif"),"w+"); //diff. btw sum \& fit;

FILE *Pdel = fopen (StrCat (fname,".deI"), "w+"); //diff. btw data \& fit;

FILE *Pstr $=$ fopen (StrCat (fname,".str"), $w+") ; / /$ strength vs order $n$;

double $x$;

for $(x=A n$. LowBoundary();

$x<=$ An.HighBoundary ()$; x+=(($ double $)$ An.BinSize ()$) / 4.0)\{$

//print out the calculated quantities for each bin of reduced width; double sqrtw = An.SqrtWeight $(x) ; \quad / /$ the original fit;

double sum $=0.0$;

//combination of orth functions;

for (int $i=0 ; i<=A n \cdot \operatorname{Max} \operatorname{Order}() ; i++)\{$

sum $+=$ An.ModeAmp $(i) *$ An.Polynomial $(x, i) *$ sqrtw;

\}

feprintf(Pfit, "\%f \%f\n", $x$, sqrtw);

fprintf(Psum, "\%f $\% f \backslash n ", x$, sum);

fprintf(Pdif, "\%f $\% f \backslash n ", x, 100.0 *$ (sum - sqrtw)/sqrtw);

\}

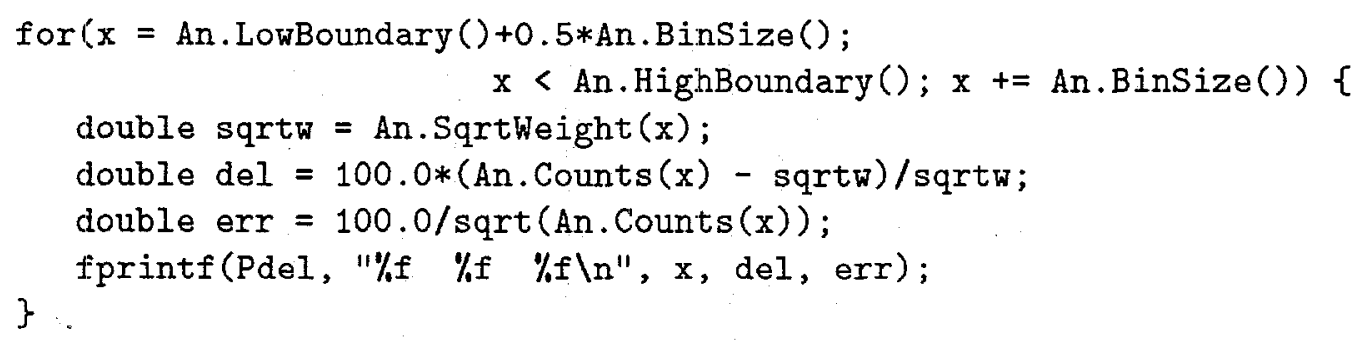




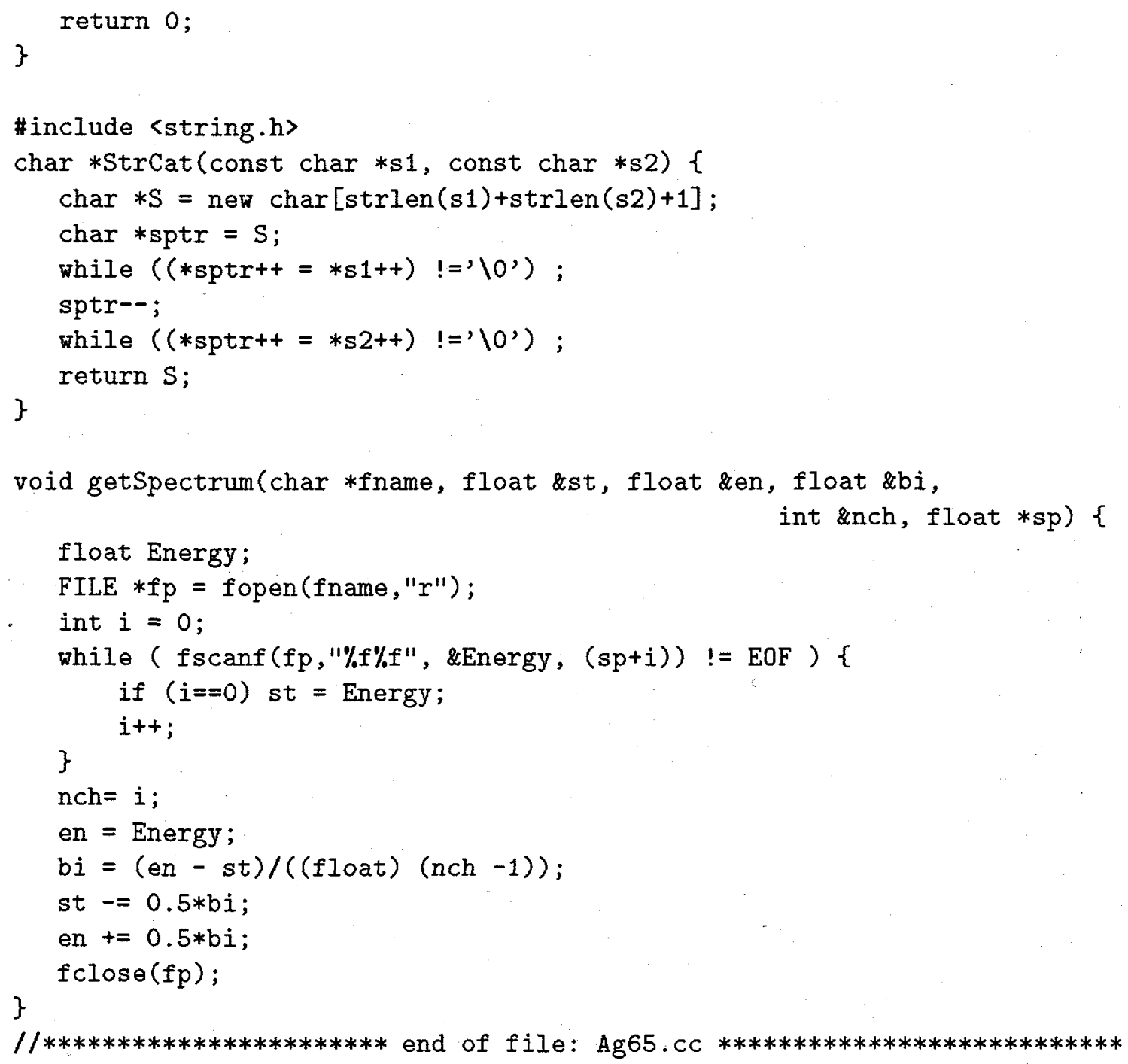


The source code of the four object classes is given below. It consists of the following files:

- class ModeAmplitudes: ModeAmplitudes.h, ModeAmplitudes.cxx

- class OrthPolyD: : OrthPolyD.h, OrthPolyD.cxx

- class OrthWeightD: OrthWeightD.h, OrthWeightD.cxx

- class Spectrum1D: Spectrum1D.h, Spectrum1D.cxx

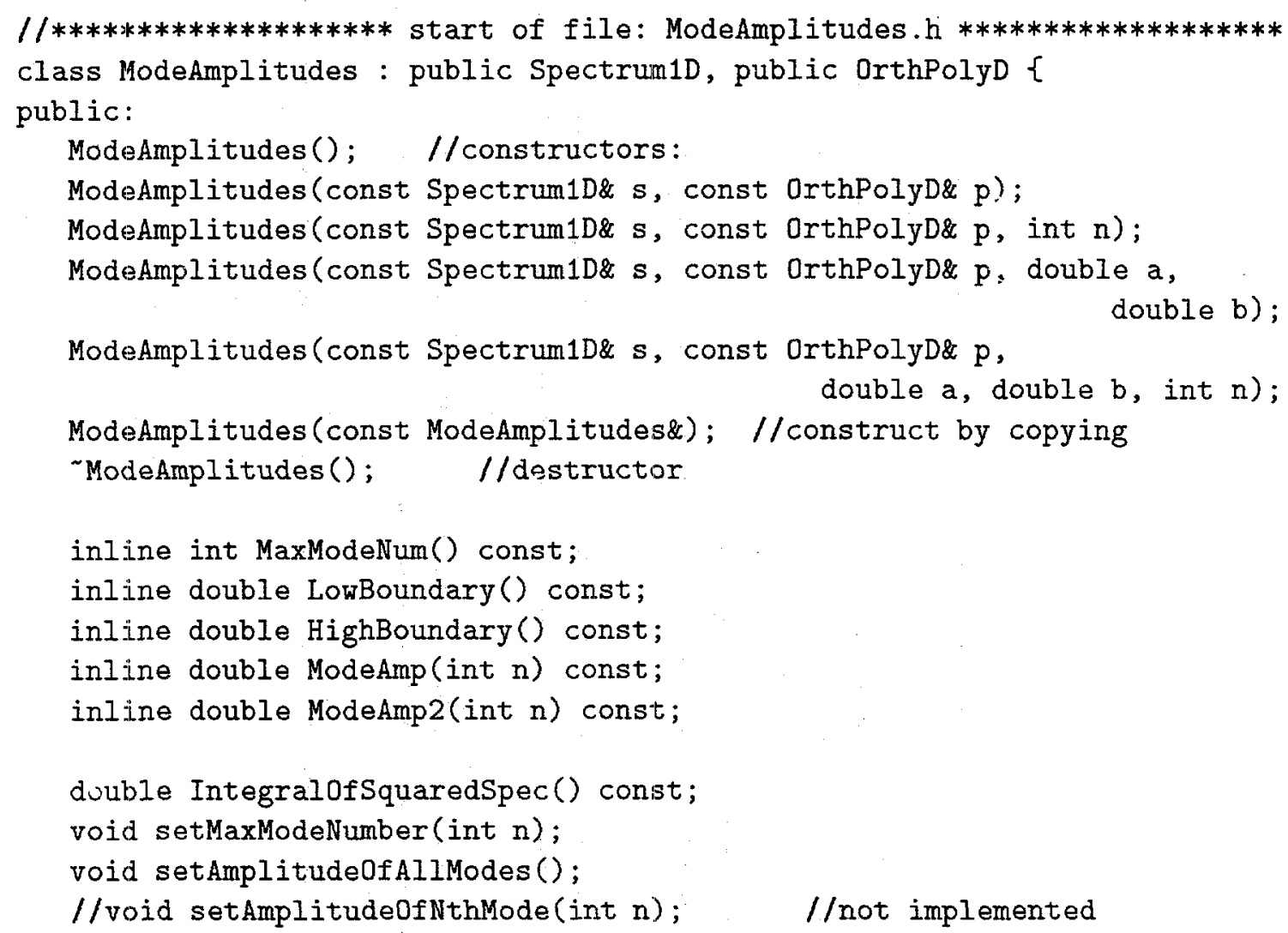




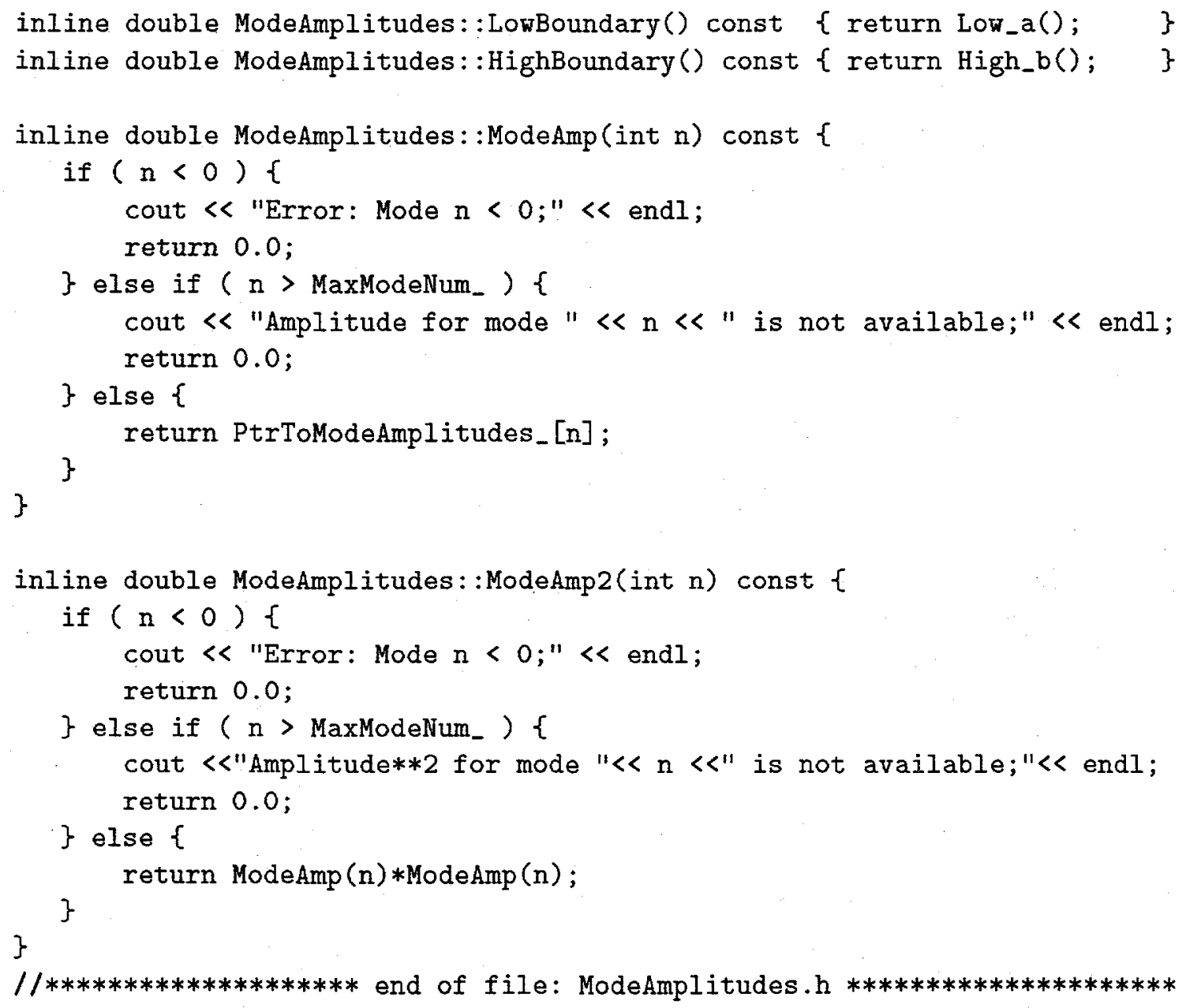

ModeAmplitudes: :ModeAmplitudes (const Spectrum1D\& s, const OrthPolyD\& $p$ ) : float $a=$ StartofSpec(); 


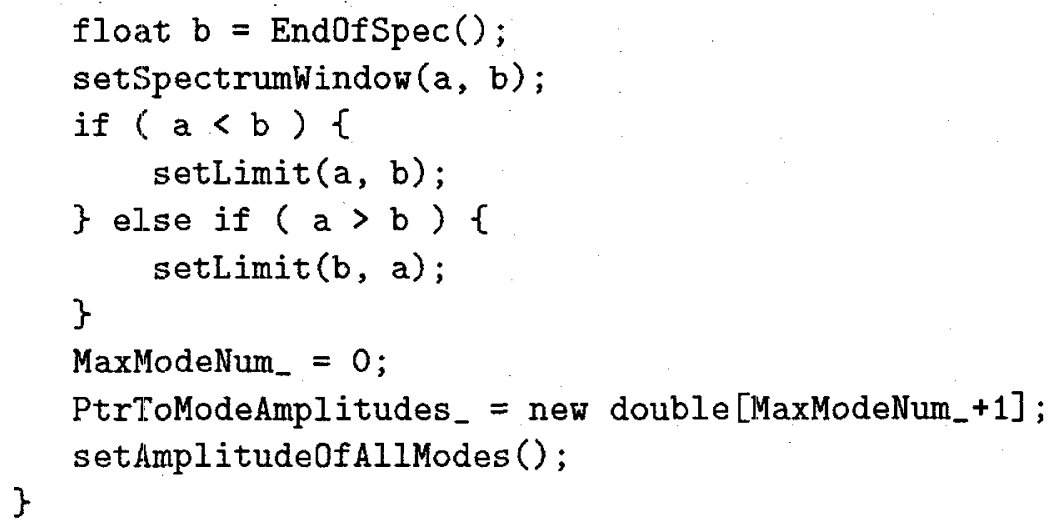

ModeAmplitudes: :ModeAmplitudes (const Spectrum1D\& $s$, const OrthPolyD\& $p$, int $n):$ Spectrum1D $(s)$, OrthPolyD $(p)\{$

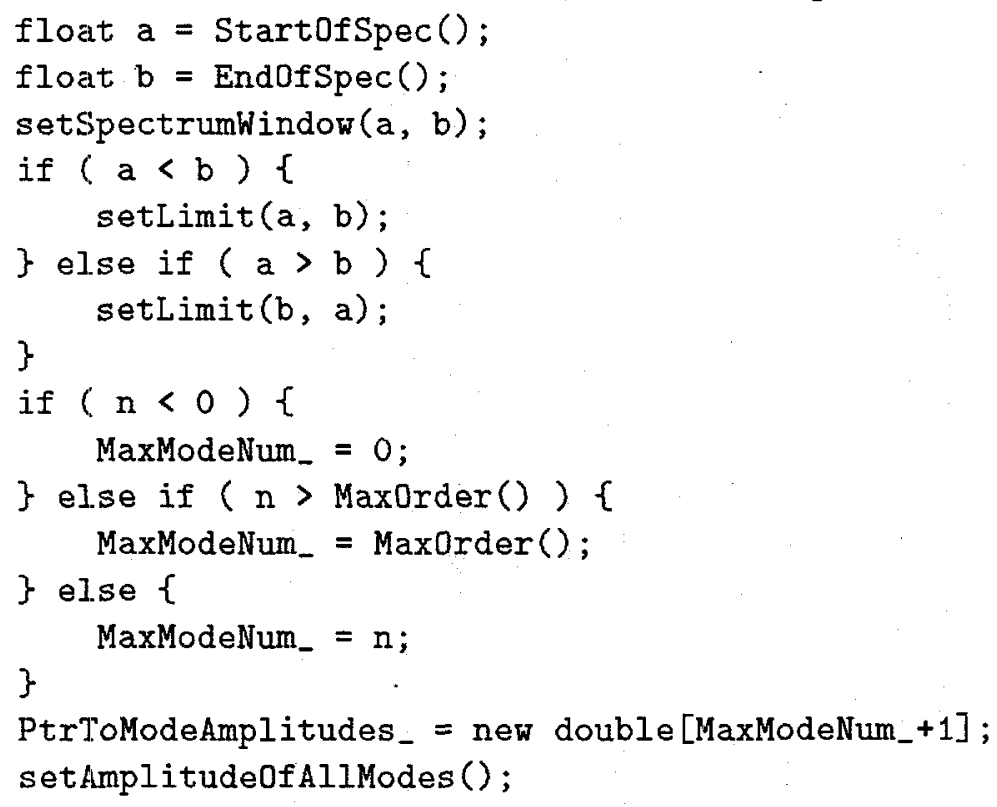




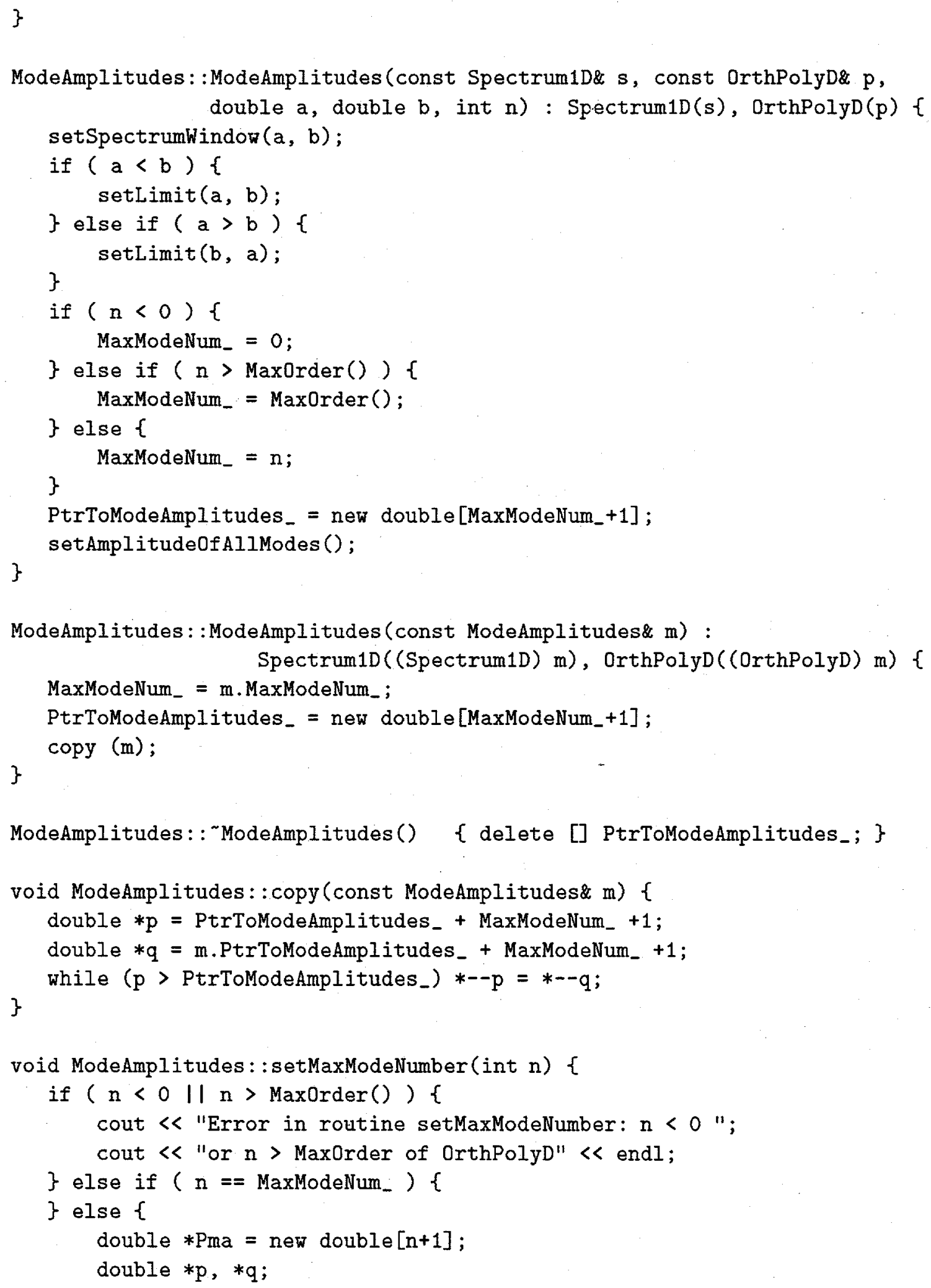




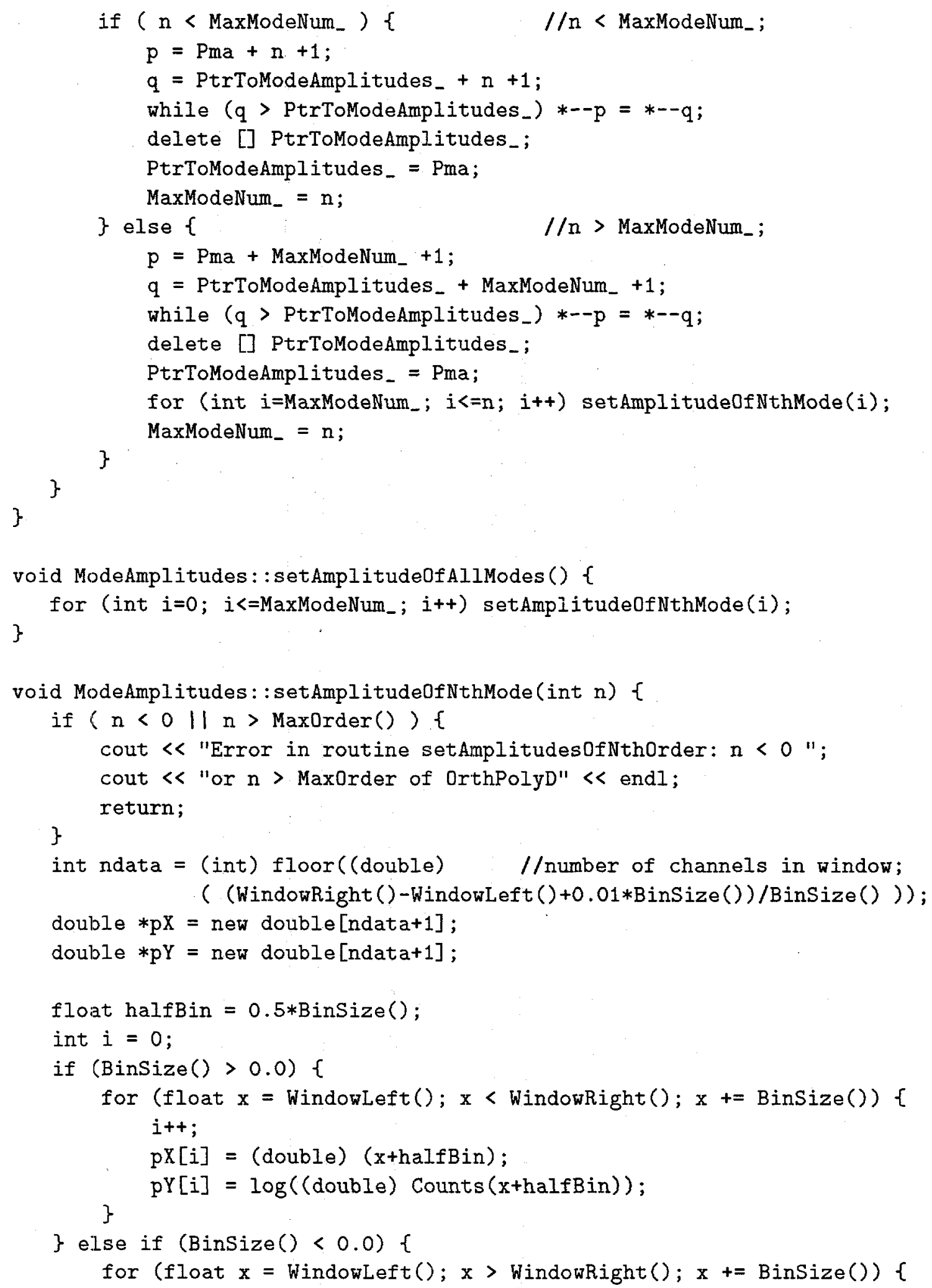




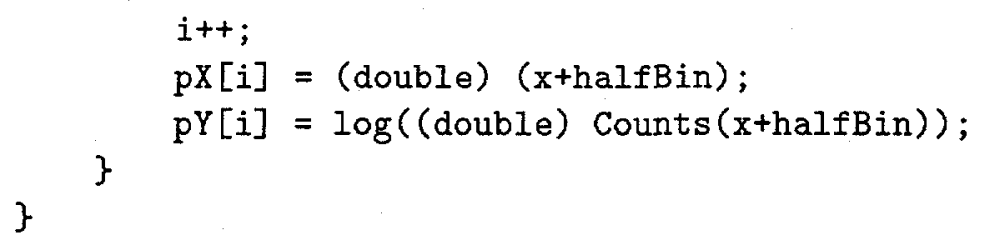




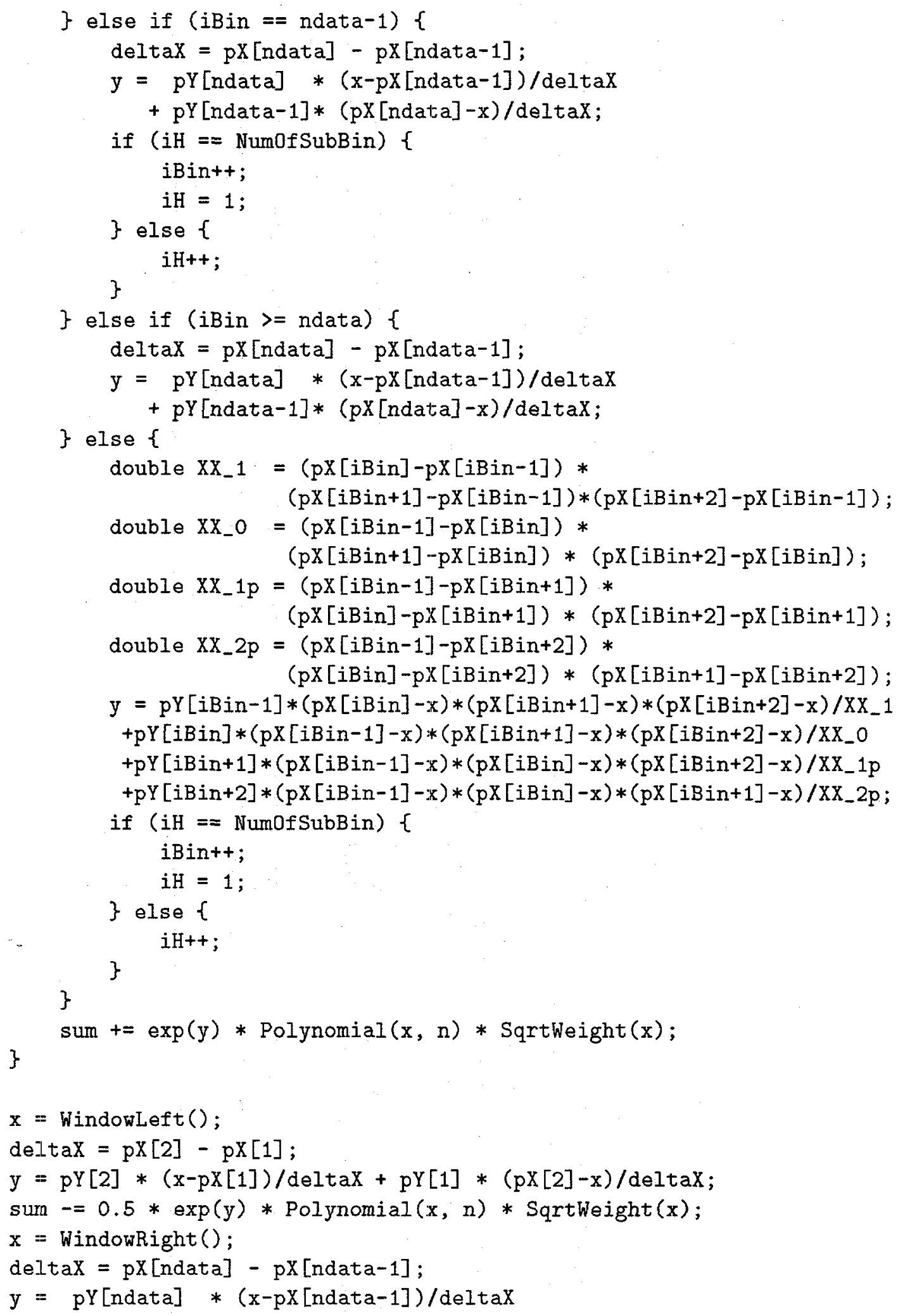




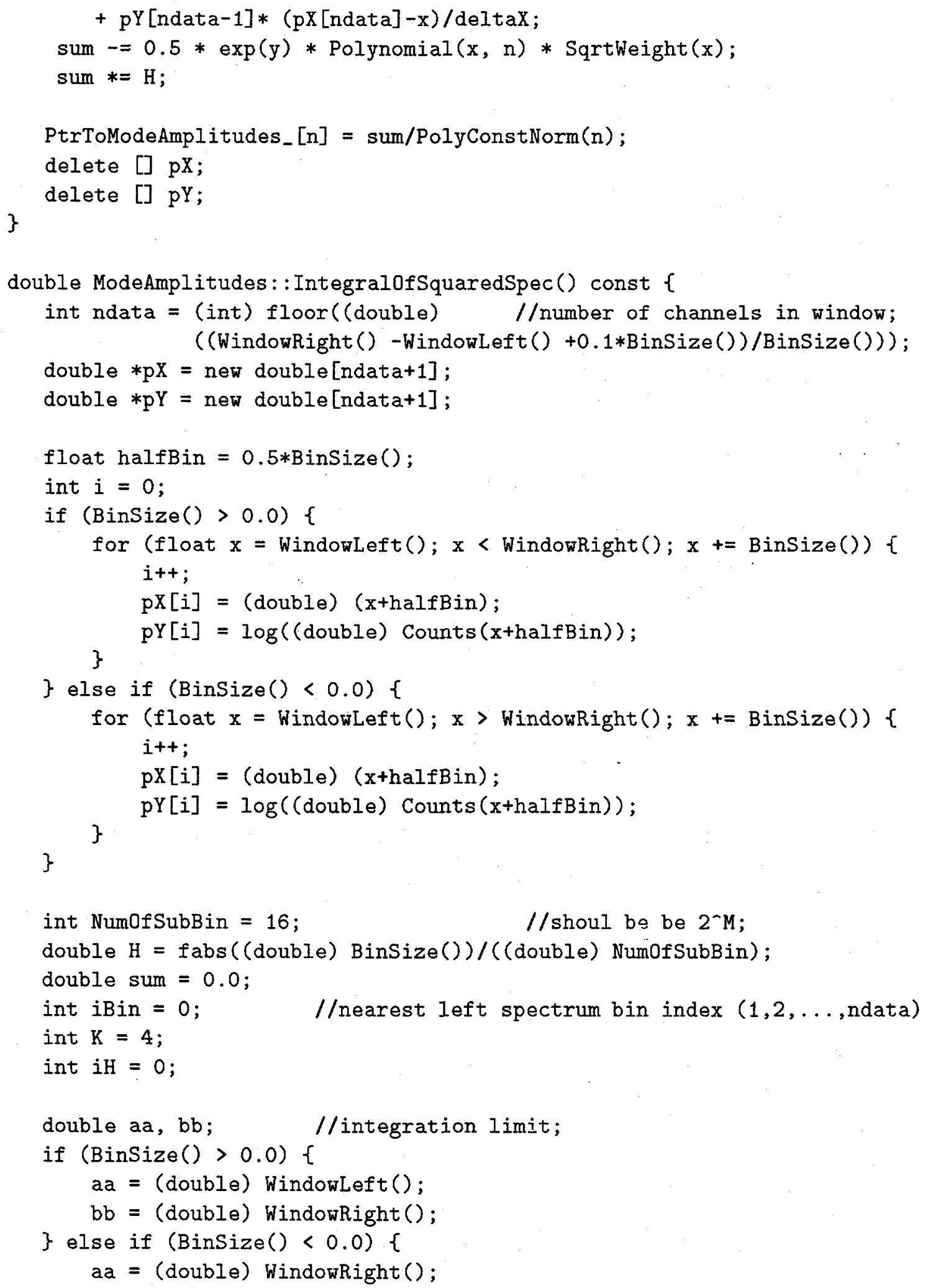




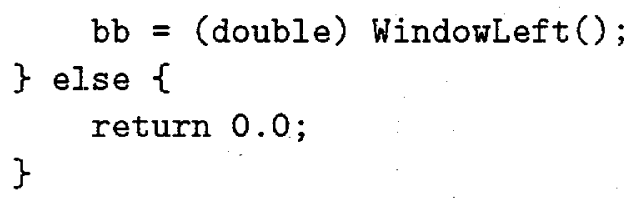




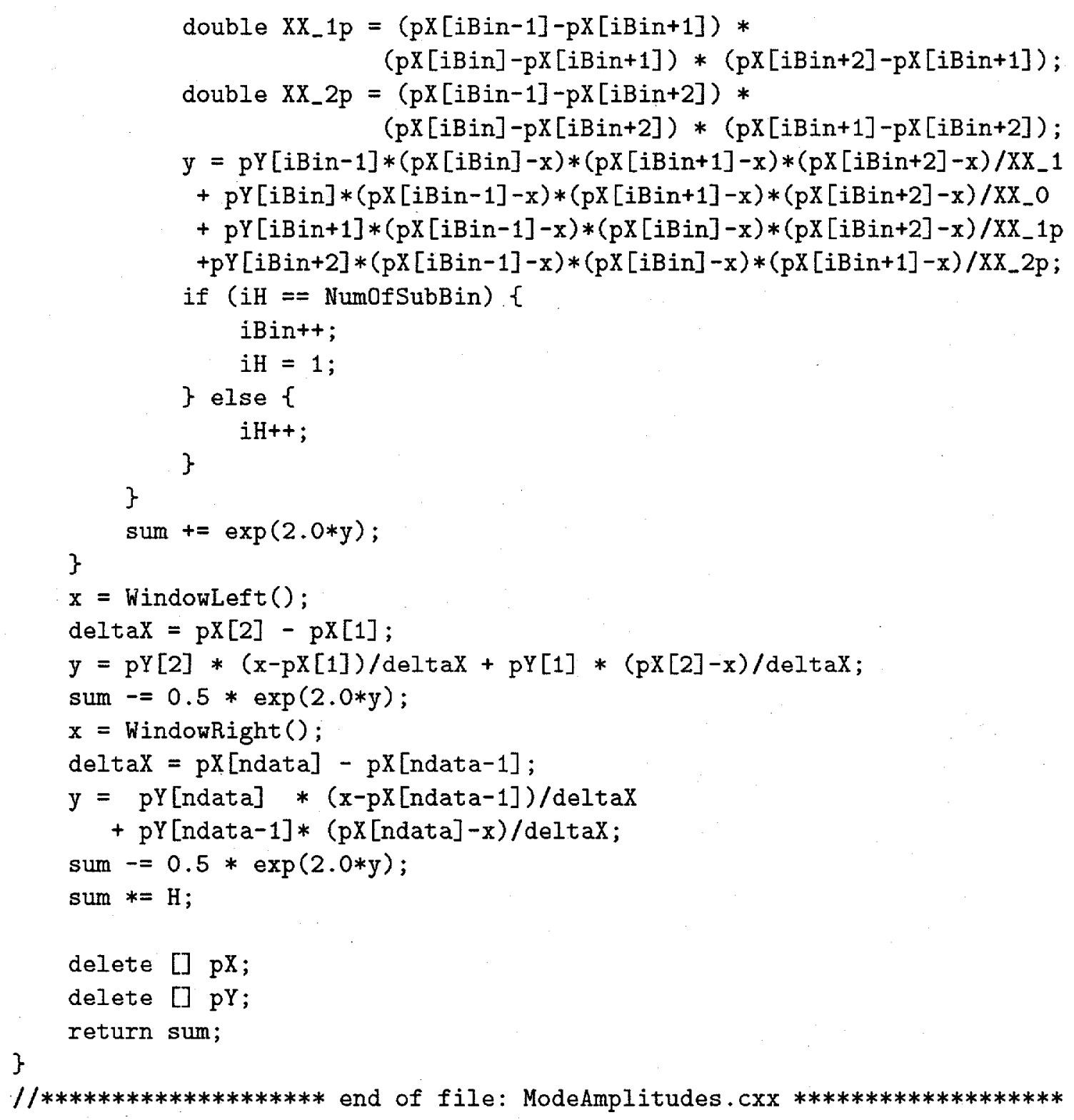

\footnotetext{
$/ / * * * * * * * * * * * * * * * * * * * * * *$ start of file: 0rthPolyD.h $* * * * * * * * * * * * * * * * * * * * * *$ class OrthPolyD : public OrthWeightD \{ public:

OrthPolyD();

OrthPolyD(OrthWeightD \&w, double $a$, double $b$, int $n$ );

OrthPolyD(OrthWeightD \&w, float $a$, float $b$, int $n$ );

OrthPolyD (const OrthPolyD \&p);

OrthPolyD(); //destructor
} 


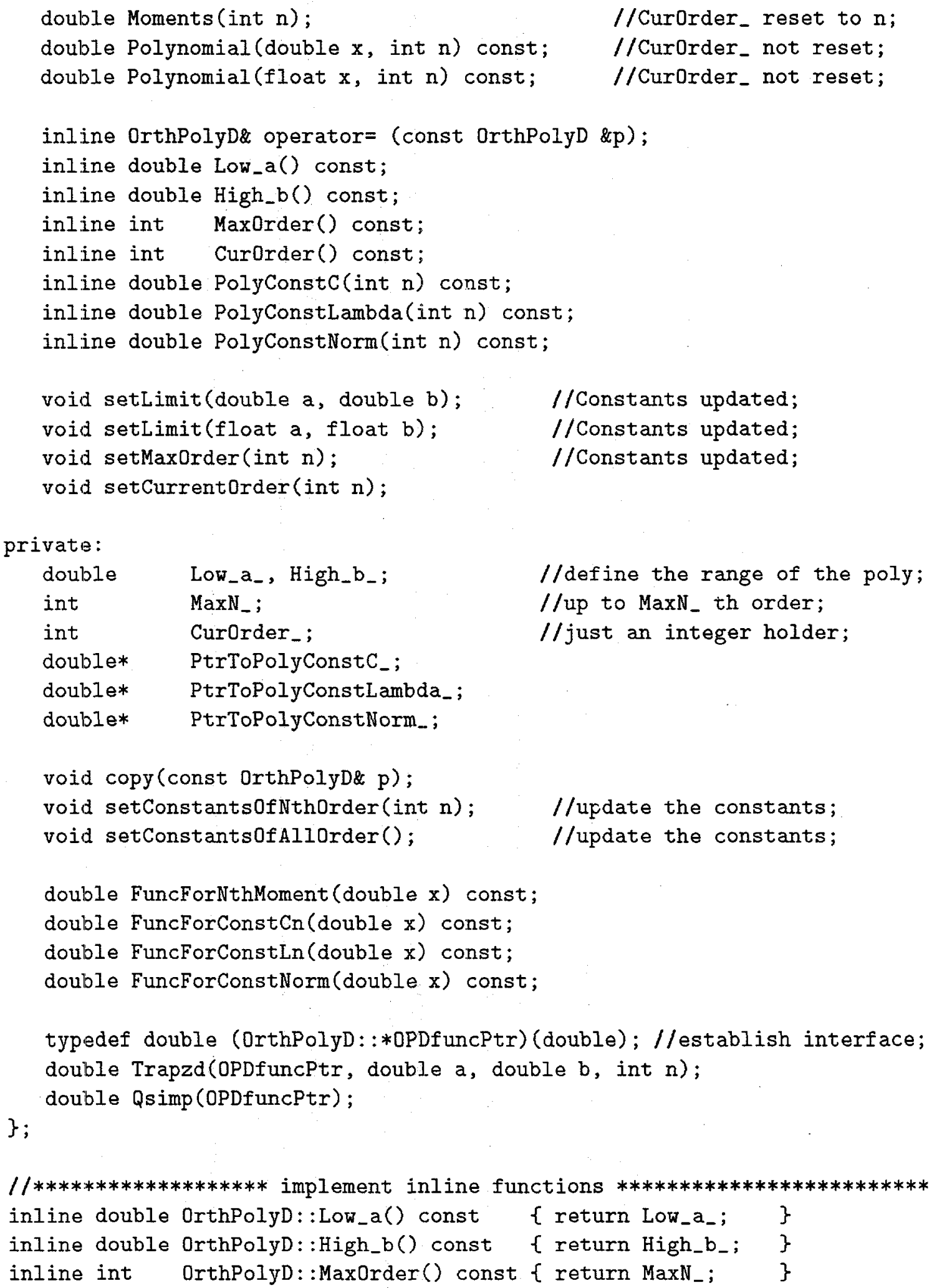




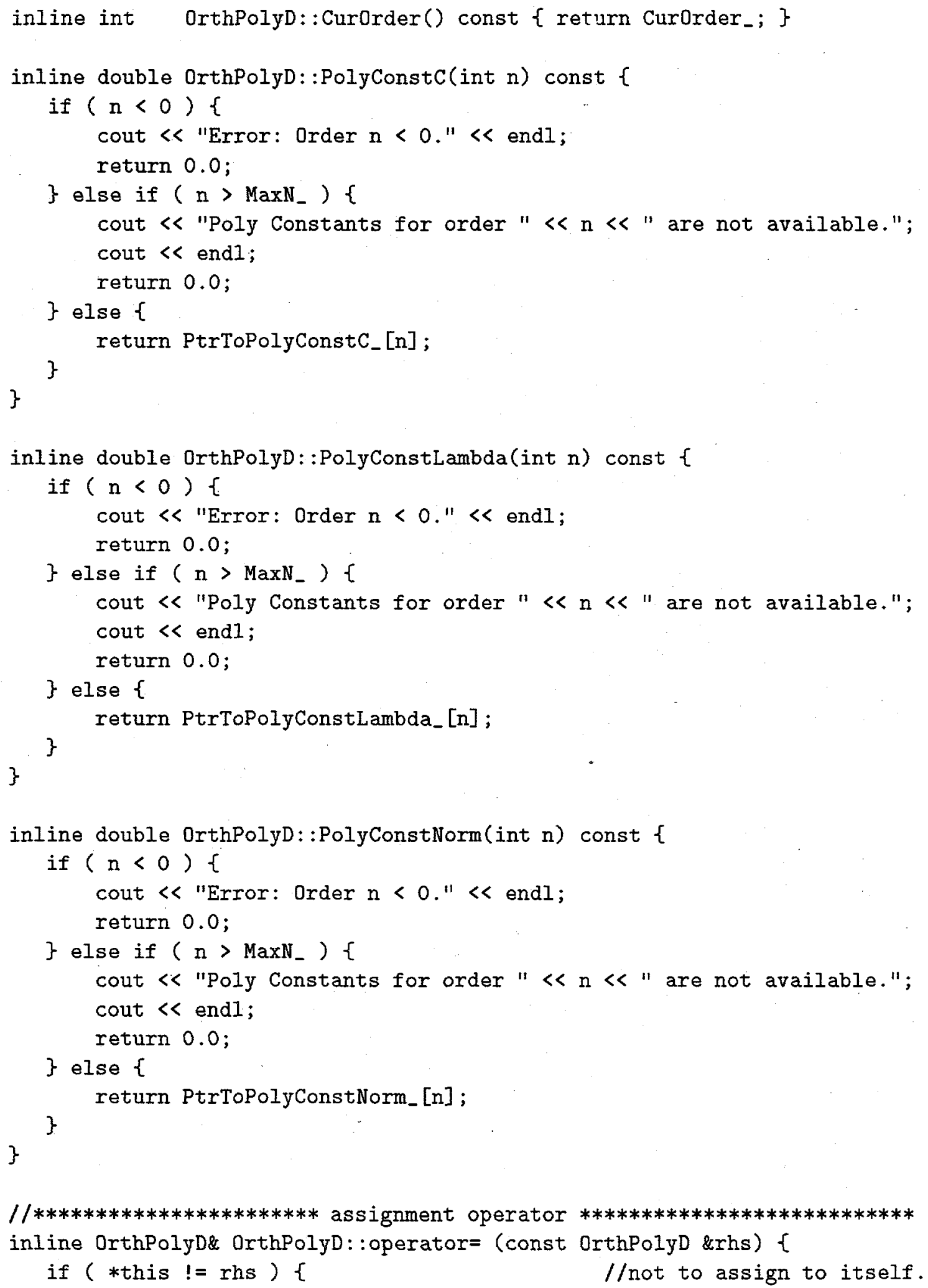




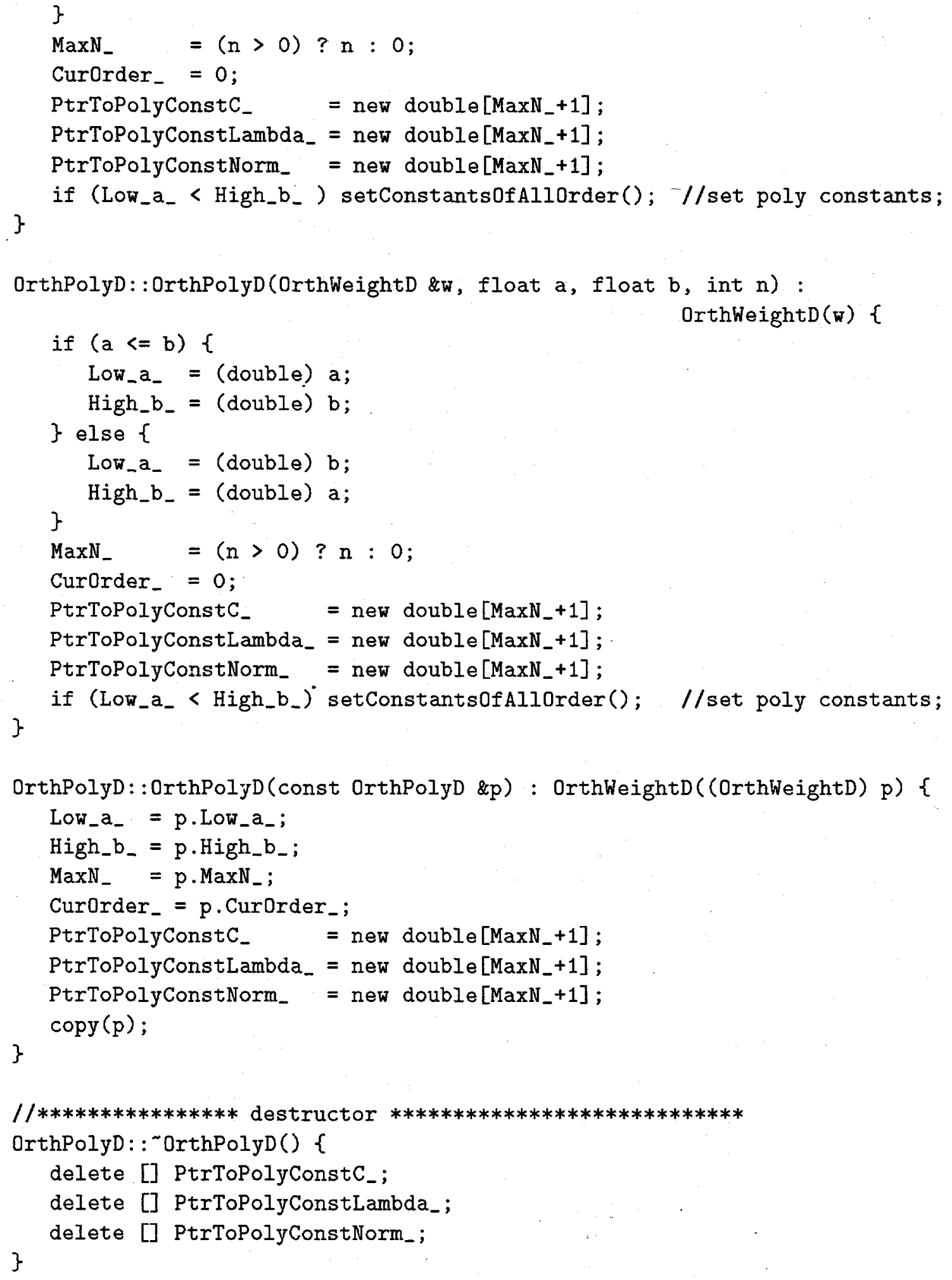




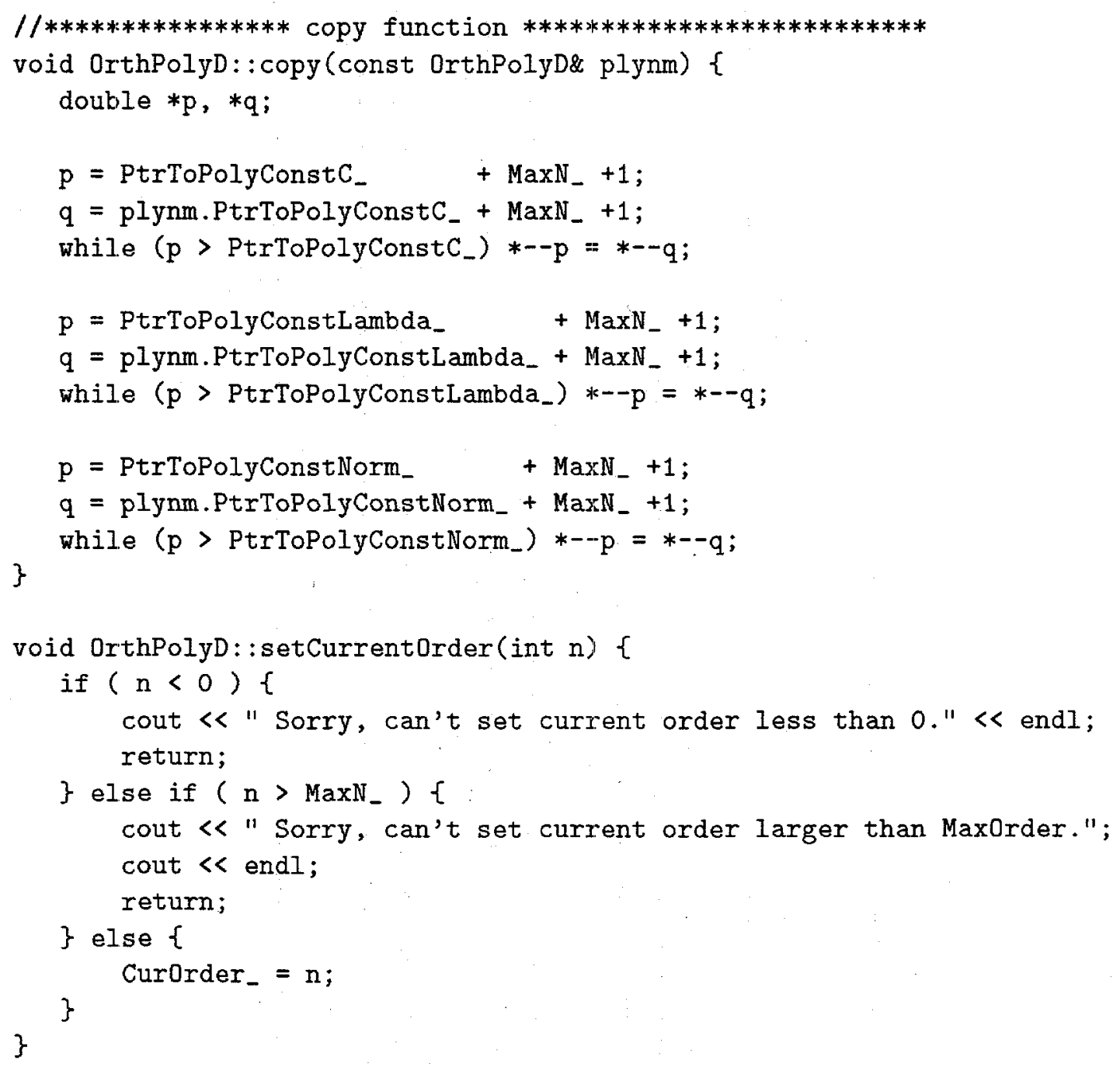




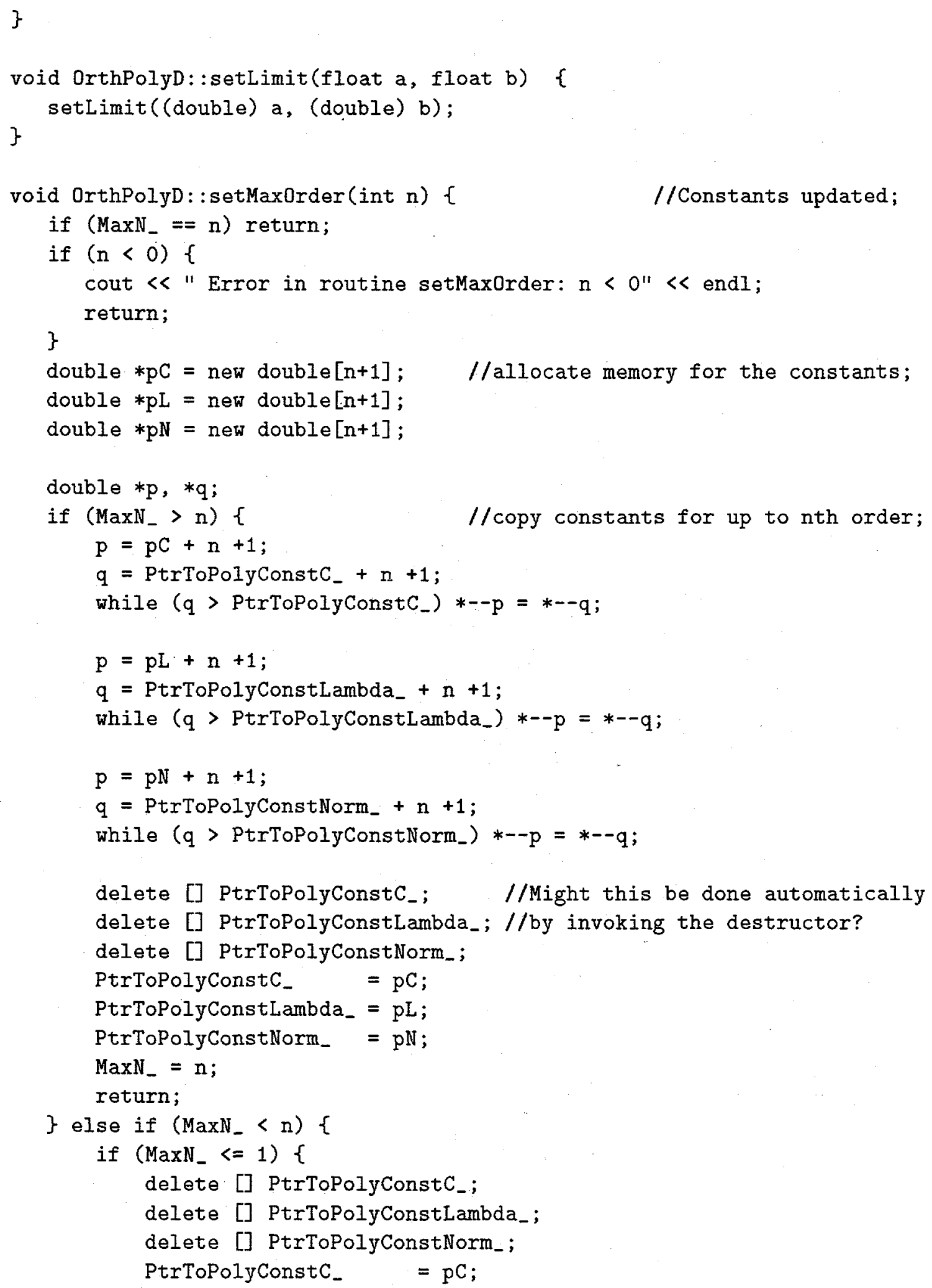




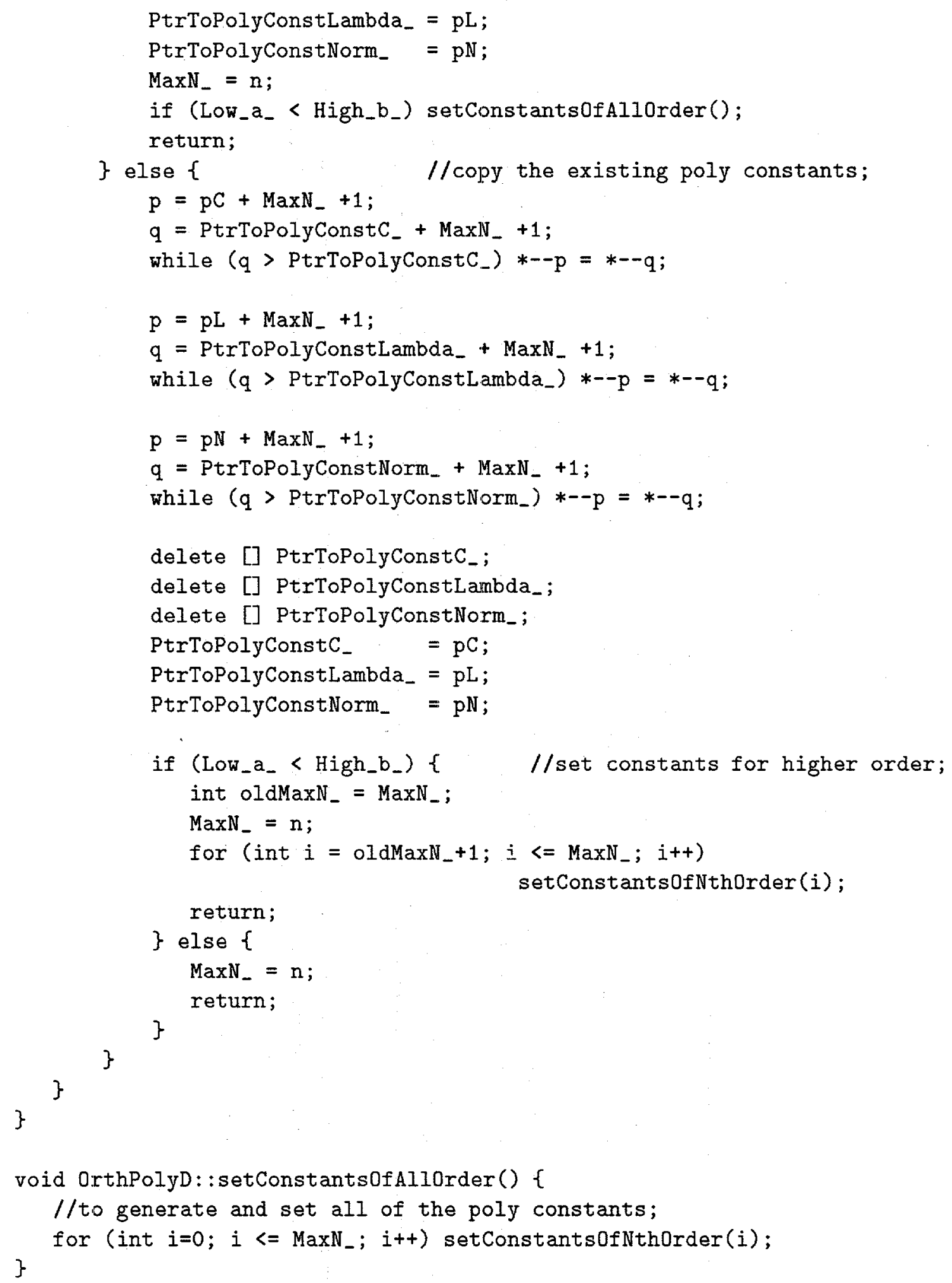




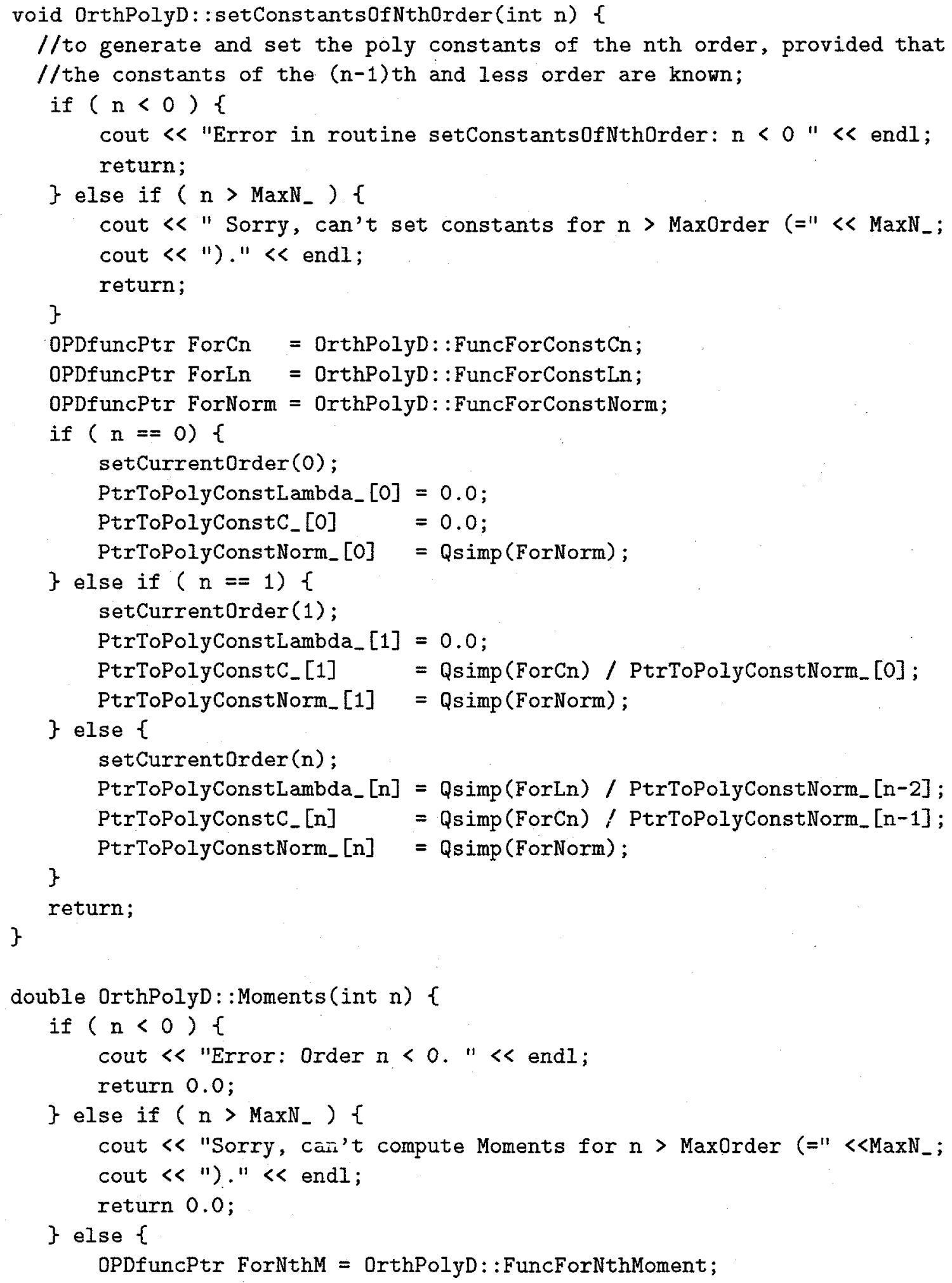




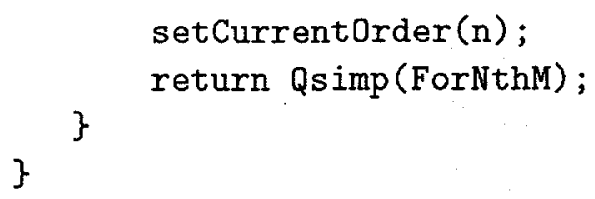




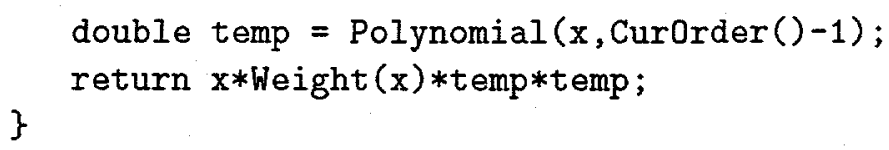

$/ / * * * * * * * * * * * * * * * * * * * * * * * * * * * * * * * * * * * * * * * * * * * * * * * * * * * * * * * * * * * * * * * * * * * * * * * * * * *$

$/ / * * * * * * * * * * * * * * * * * * * * * * * * * * * * * * * * * * * * * * * * * * * * * * * * * * * * * * * * * * * * * * * * * * * * * * * * * *$ double OrthPolyD::Trapzd(OPDfuncPtr func, double $a$, double b, int $n$ ) \{

1* This routine computes the nth stage of refinement of an extended

* trapzoidal rule. func is input as a pointer to the function to be

* integrated between $a$ and $b$, also input. When called with $n=1$,

* the routine returns the crudest estimate of int $f(x) d x[a, b]$.

* Subsequent calls with $n=2,3 \ldots$ (in that sequential order) will improve

* the accuracy of $s$ by adding $2^{\sim}(n-2)$ additional interior points.

* Adapted from [Pres 92].

$* 1$

double $x$, tnm, sum, del;

static double s;

int it, $j$;

if $(n==1)\{$

return $(s=0.5 *(b-a) *(($ this->*func $)(a)+($ this->*func $)(b)))$;

\} else \{

for $(i t=1, j=1 ; j<n-1 ; j++)$ it $\ll<=1$;

tnm $=i t$;

del $=(b-a) / \mathrm{tnm} ; \quad / /$ This is the spacing of

$\mathrm{x}=\mathrm{a}+0.5 *$ del; $\quad / /$ the points to be added;

for (sum=0.0,j=1; j<=it; $j++, x+=d e l$ ) sum $+=$ (this $->*$ func) ( $x$ );

$\mathrm{s}=0.5 *(\mathrm{~s}+(\mathrm{b}-\mathrm{a}) * \operatorname{sum} / \mathrm{tnm}) ; / /$ This replaces a by its refined value return $s$;

\}

\}

double OrthPolyD::Qsimp (OPDfuncPtr func) \{

/* float qsimp(float (*func)(float), float a, float b)

* Returns the integral of the function func from a to $b$. 
double Weight(float $x$ ) const;

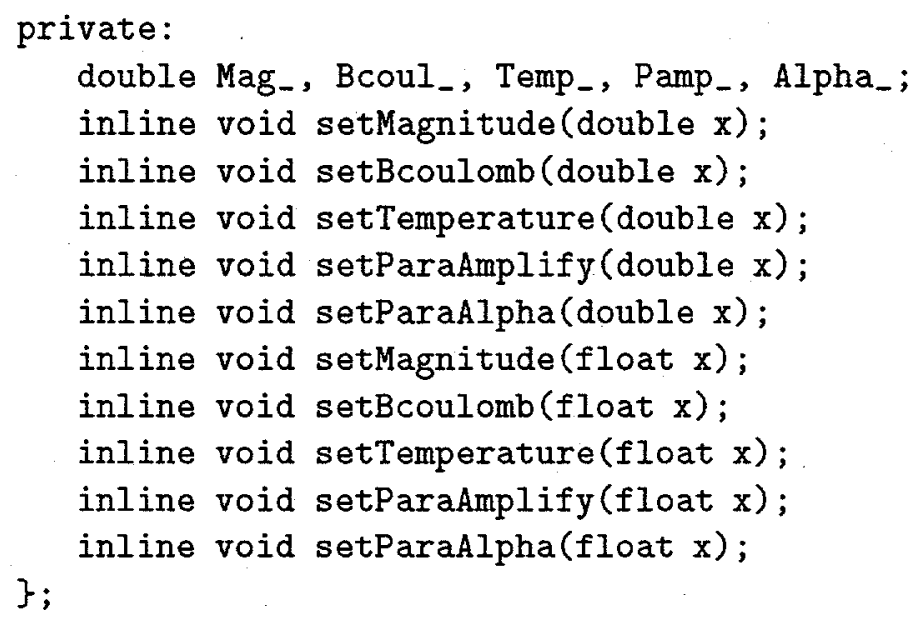

//constructors:

inline OrthWeightD::OrthWeightD():

Mag_(0.0), Bcoul_(0.0), Temp_(0.0), Pamp_(0.0), Alpha_(0.0)\{\}

inline OrthWeightD::OrthWeightD(double P1, double P2, double P3, double P4, double P5) : Mag_(P1), Bcoul_(P2), Temp_(P3), Pamp_(P4), Alpha_(P5) \{\}

inline OrthWeightD::OrthWeightD(float P1, float P2, float P3, float P4, float P5) : Mag_((double) P1), Bcoul_((double) P2), Temp_((double) P3), Pamp_((double) P4), Alpha_((double) P5) \{\}

inline OrthWeightD::OrthWeightD (const OrthWeightD \&w) :

Mag_(w.Mag_), Bcoul_(w.Bcoul_), Temp_(w.Temp_), Pamp_(w.Pamp_), Alpha_(w.Alpha_) \{\}

//member accessors:

inline double CrthWeightD::Magnitude() const inline double OrthWeightD::Bcoulomb() const inline double OrthWeightD: :Temperature() const \{ return Mag-; \} $\{$ return Bcoul_; \} inline double OrthWeightD: :ParaAmplify() const $\{$ return Temp_; \} inline double OrthWeightD: :ParaAlpha() const $\{$ return Pamp_; \} $\{$ return Alpha_; \}

//set values

inline void OrthWeightD: :setMagnitude(double $x$ ) $\{$ Mag- $=x ;\}$ inline void OrthWeightD: : setBcoulomb(double $x$ ) $\quad\left\{\right.$ Bcoul_ $\left._{-}=x ;\right\}$ inline void OrthweightD: :setTemperature (double $x$ ) $\{$ Temp $=x ;\}$ inline void OrthWeightD: :setParaAmplify (double $x$ ) $\left\{\right.$ Pamp $\left._{-}=x ;\right\}$ inline void OrthweightD: :setParaAlpha(double $x$ ) $\quad\{$ Alpha $=x ;\}$ 


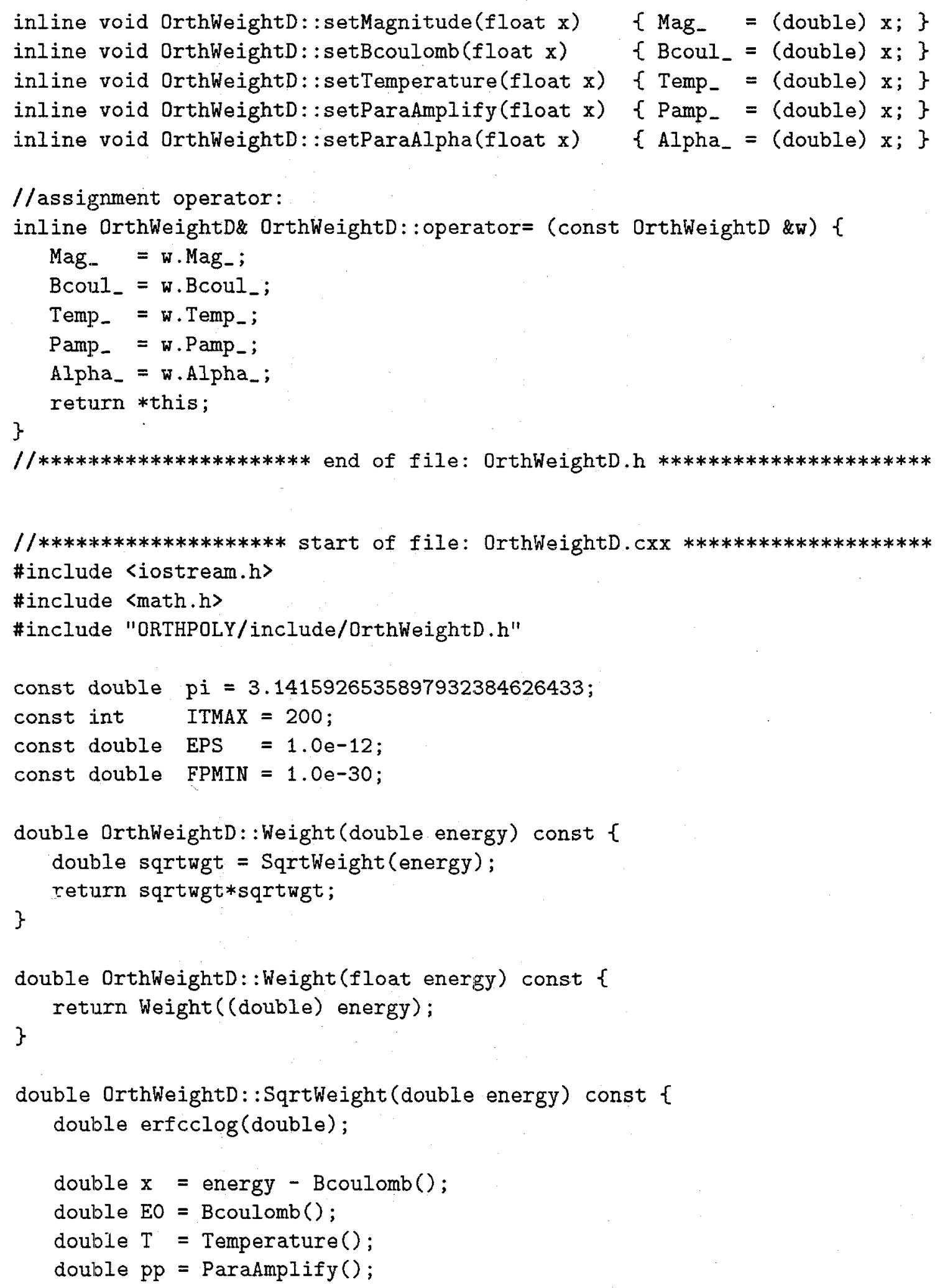




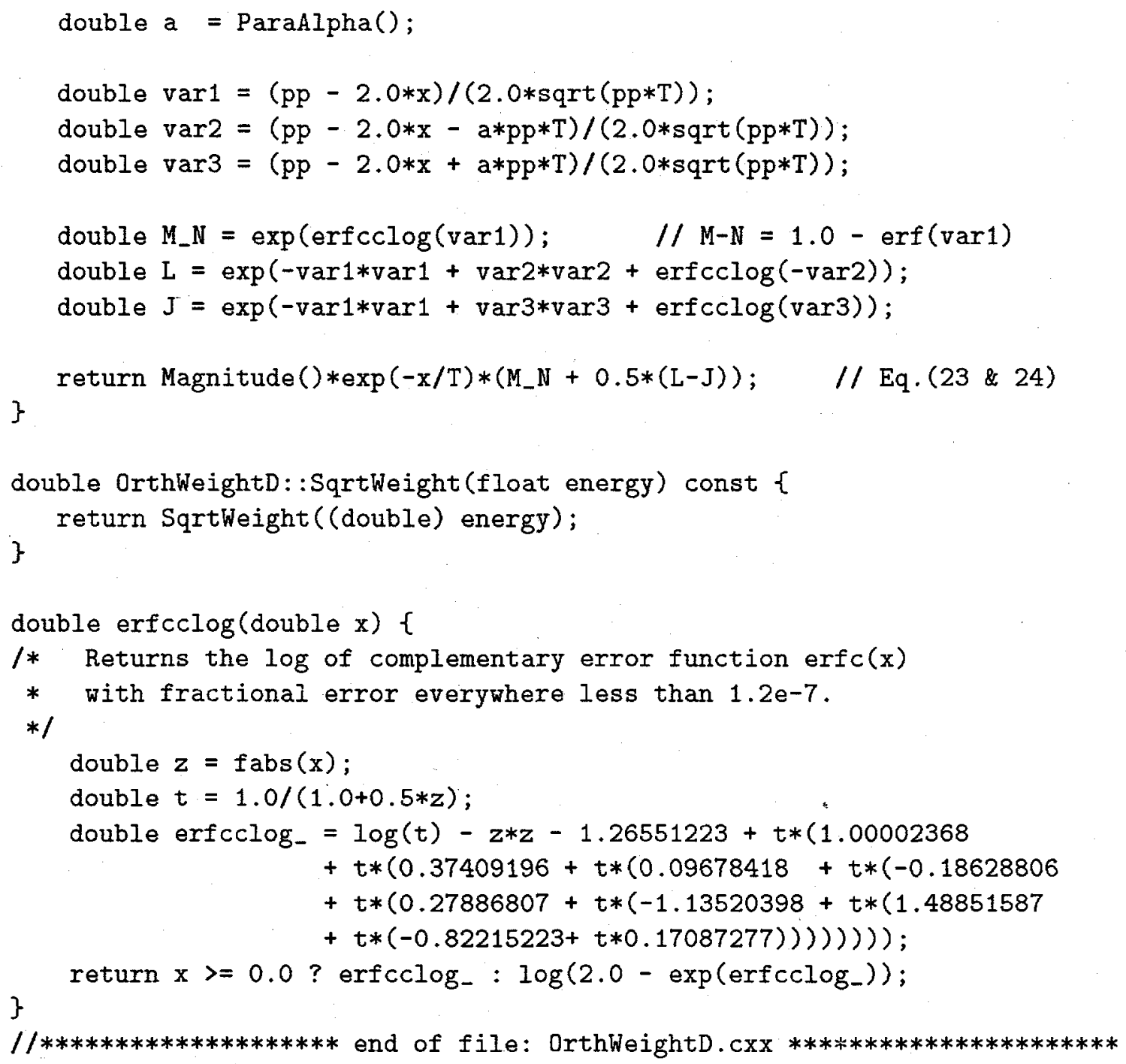


inline float Start0fSpec() const;

inline float EndofSpec() const;

inline float BinSize() const;

inline float Windowleft() const;

inline float WindowRight() const;

inline float $*$ PointerToData() const;

//functions that return spectrum properties:

inline float Counts (int) const;

inline float Counts (float) const;

inline float Counts (double) const;

inline float CountsInWindow (int, int) const;

inline float CountsInWindow(float, float) const;

inline float CountsInWindow (double, double) const;

inline float CountsInWindow() const;

inline float TotalCounts() const;

inline void setSpectrumWindow(float \&a, float \&b);

inline void setSpectrumWindow (double \&a, double \&b);

// Spectrum1D\& compress (const Spectrum1D\&);

$/ /$ search for a list of peaks;

$/ /$ window facilities;

// void PrintSpectrum1D();

protected:

float Window_Left_, Window_Right_; //Spectrum window limit;

private:

int Num0fChannels; ;

float Start0fSpec, End0fSpec, BinSize ;

float* ptr_to_data_;

void copy (const Spectrum1D\& s);

\};

$/ / * * * * * * * * * * * * * * * * * * * * *$ implement inline functions $* * * * * * * * * * * * * * * * * * * * * * *$

//member accessors:

inline int Spectrum1D::Num0fChannels() const \{return Num0fChannels_; \}

inline float Spectrum1D::StartOfSpec() const \{return Start0fSpec_; \}

inline float Spectrum1D::EndOfSpec() const $\quad$ return EndOfSpec_; \}

inline float Spectrum1D::BinSize() const \{return BinSize; ;

inline float Spectrum1D: :WindowLeft () const $\quad\{$ return Window_Left_; \}

inline float Spectrum1D::WindowRight() const $\{$ return Window_Right_; $\}$

inline float* Spectrum 1D: :PointerToData() const $\{$ return ptr_to_data_; $\}$ 
//functions that return spectrum properties:

inline float Spectrum1D::Counts(int chn) const \{

if (ptr_to_data_ $!=0 \& \& \mathrm{chn}>=0 \& \& \mathrm{chn}<$ Num0fChannels $)\{$ return $*$ (ptr_to_data_+chn);

\} else \{

return 0.0 ;

\}

\}

inline float Spectrum 1D::Counts (float $x$ ) const \{ int $c h n=($ int $)$ floor $\left((\right.$ double $)\left(\left(x-\right.\right.$ Start0fSpec $\left._{-}\right) /$BinSize_ $\left.\left._{-}\right)\right)$;

return Counts (chn);

\}

inline float Spectrum1D::Counts (double $x$ ) const \{

int chn $=$ (int), floor $((x-(($ double $)$ Start0fSpec_ $)) /(($ double $)$ BinSize_s $))$; return Counts (chn);

\}

inline float Spectrum1D: : CountsInWindow (int $n 1$, int $n 2$ ) const \{

if ( $p t r_{-}$to_data $==0$ ) return 0.0 ;

if $(\mathrm{n} 1>\mathrm{n} 2)\{$

//empty spectrum;

int temp $=\mathrm{n} 1$;

//swap $\mathrm{n} 1$ and $\mathrm{n} 2$;

$\mathrm{n} 1=\mathrm{n} 2$;

$\mathrm{n} 2$ = temp;

\}

if ( $\mathrm{n} 1>=$ Num0fChannels_ $\| \mathrm{n} 2<0)$ return $0.0 ; / /[\mathrm{n} 1, \mathrm{n} 2]$ is not in

if $(\mathrm{n} 1<0) \mathrm{n} 1=0$;

//the spectrum range.

if ( $\mathrm{n} 2>=$ Num0fChannels_) $\mathrm{n} 2$ = Num0fChannels -1 ;

float sum $=0.0$;

$/ / \mathrm{n} 1=\mathrm{n} 2 \mathrm{OK}$

float $*$ begin $=$ ptr_to_data $+\mathrm{n} 1$;

float $*$ end $=$ ptr_to_data $+\mathrm{n} 2+1$;

while (end $!=$ begin) sum $+=*--$ end;

return sum;

\}

inline float Spectrum1D: : CountsInWindow(float $a$, float $b$ ) const \{

if (ptr_to_data $==0$ ) return $0.0 ; \quad$ //empty spectrum;

int $\mathrm{n} 1=$ (int) floor ( (double) $\left((a-\right.$ StartofSpec_ $) /$ BinSize $\left.\left._{-}\right)+0.5\right)$;

int $\mathrm{n} 2=$ (int) floor $($ (double $)\left(\left(\mathrm{b}-\right.\right.$ Start0fSpec_- $\left._{-}\right) /$BinSize $\left.\left._{-}\right)+0.5\right)$;

return CountsInWindow(n1, n2-1);

\}

inline float Spectrum1D: : CountsInWindow(double $a$, double b) const \{ 


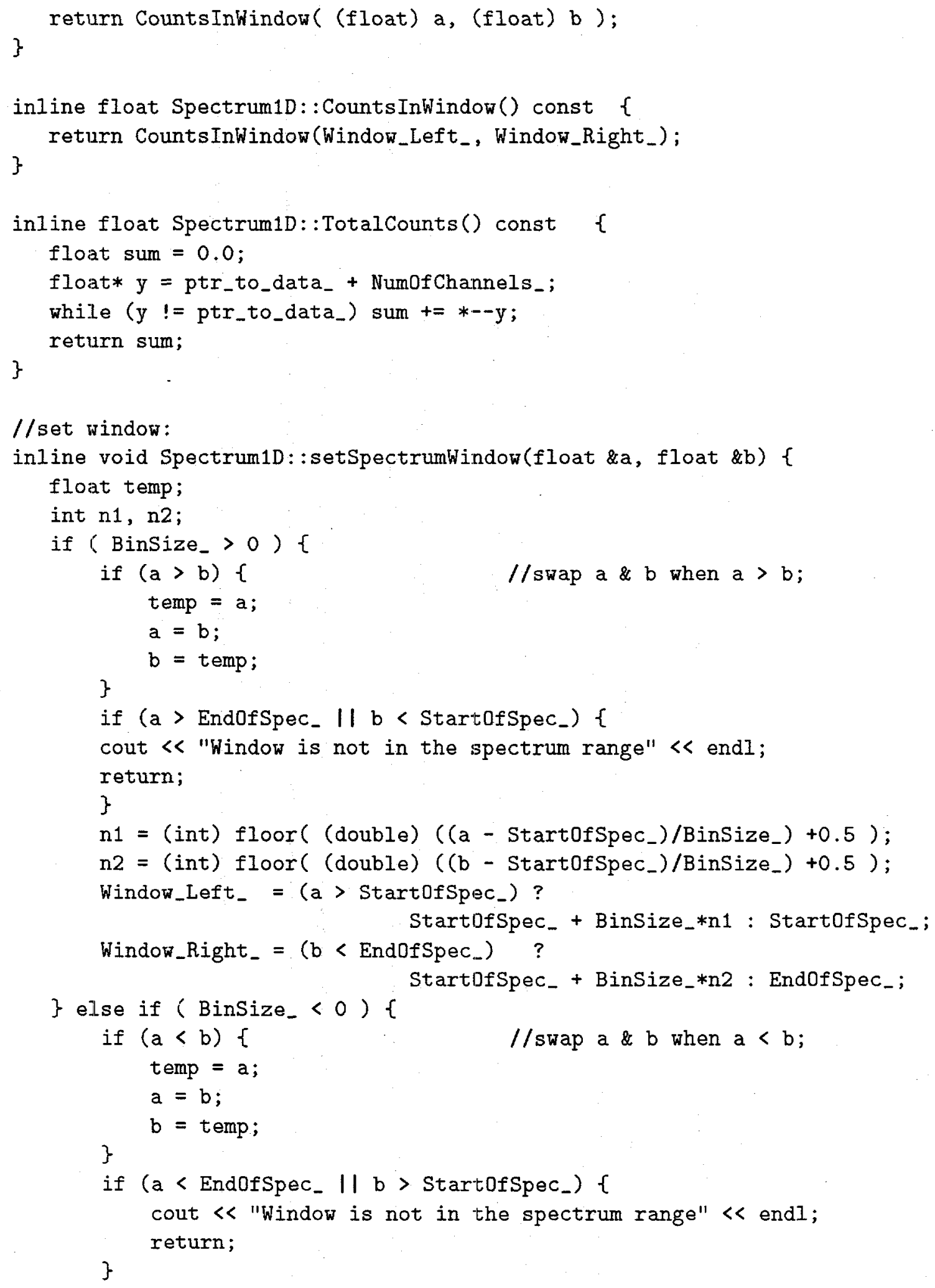




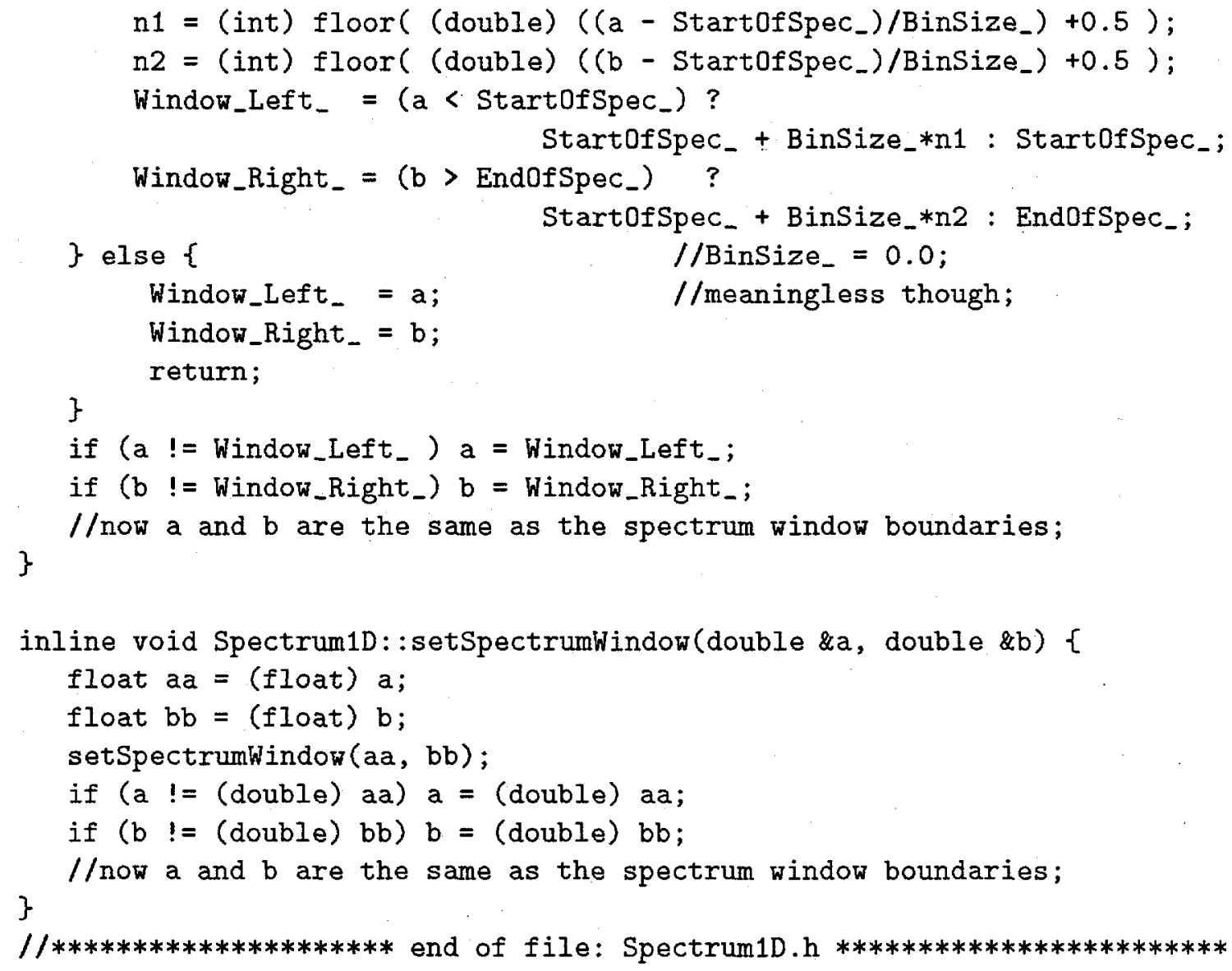




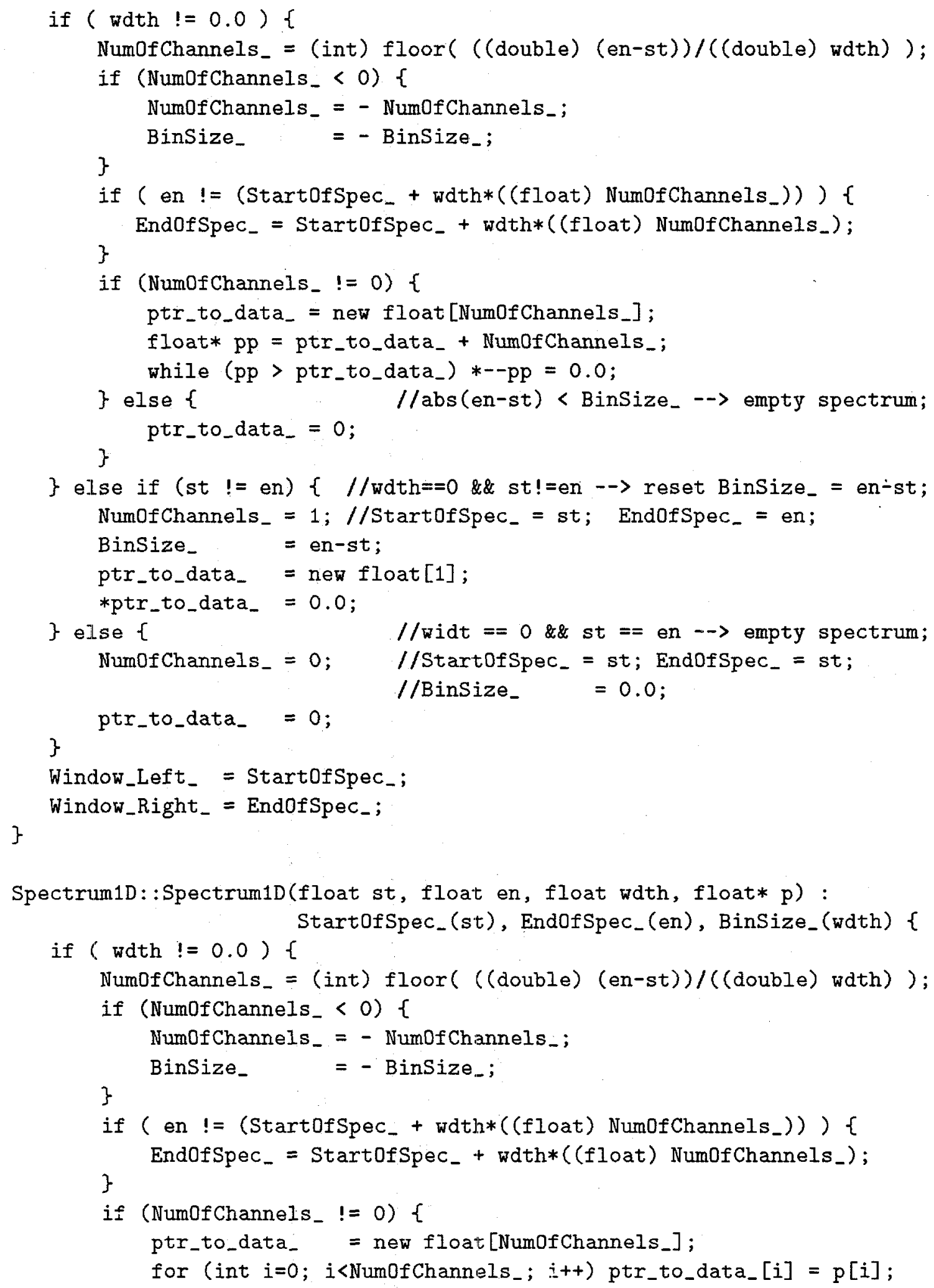




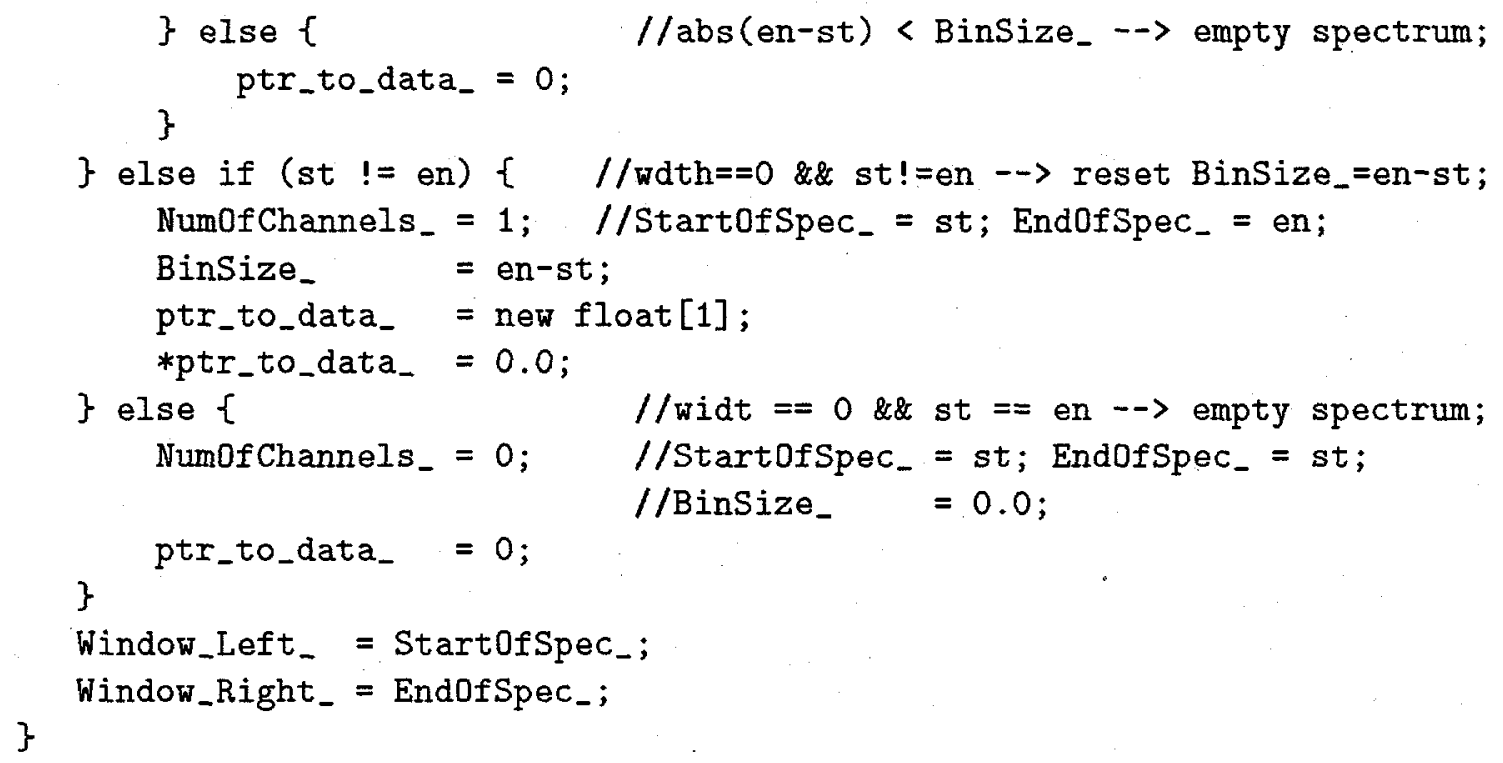




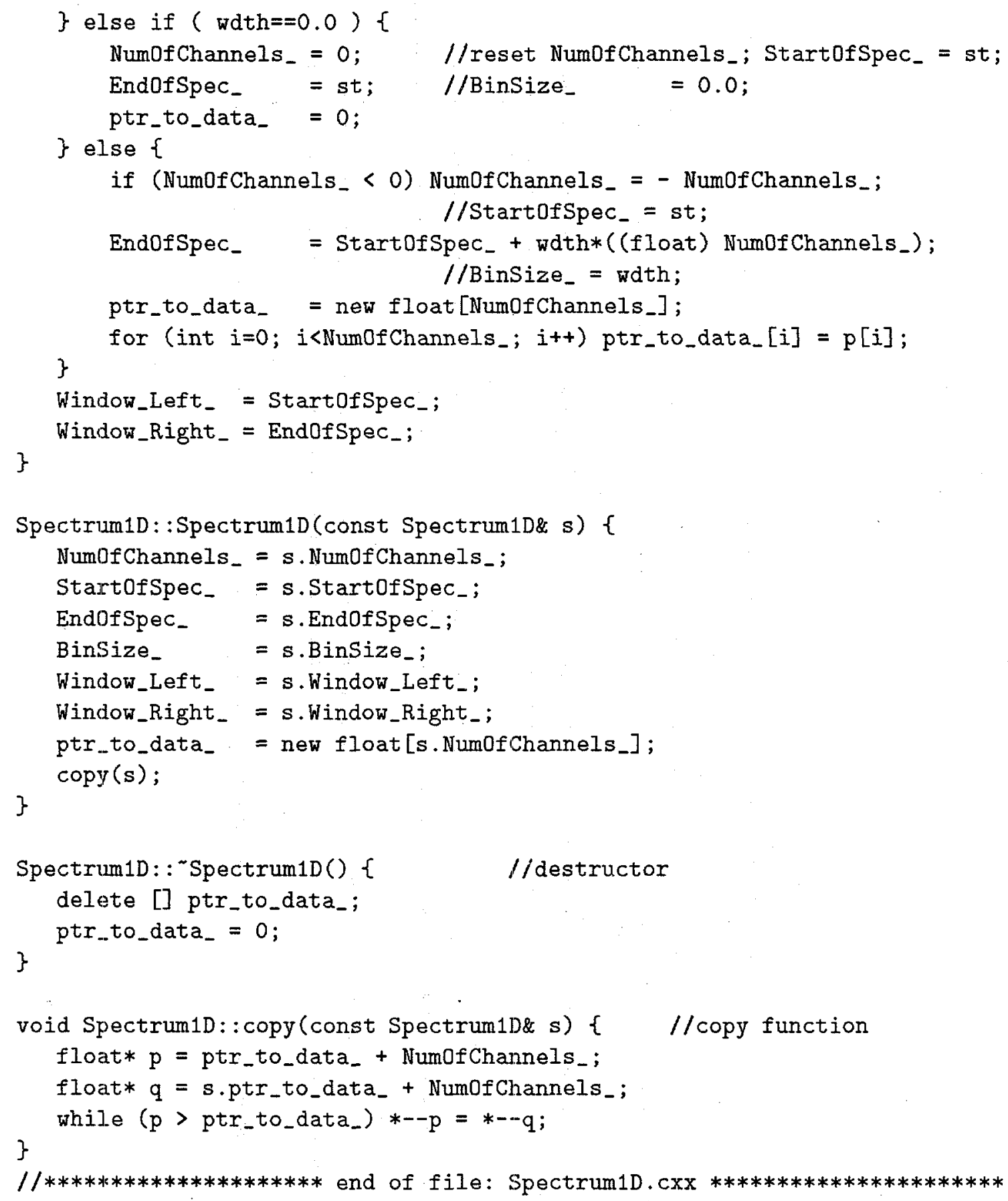

